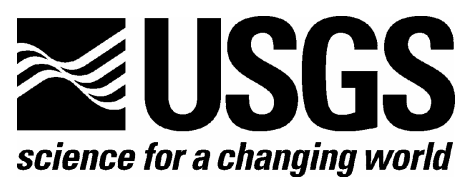

\title{
The Virtual Data Center Tagged-Format Tool- Introduction and Executive Summary
}

By John R. Evans ${ }^{1}$, Melinda Squibb², Christopher D. Stephens ${ }^{1}$, W.U. Savage ${ }^{1}$, Hamid Haddadi3 ${ }^{3}$, Charles A. Kircher $^{4}$, and Mahmoud M. Hachem ${ }^{5}$

Open-File Report 2008-1327

\section{U.S. Department of the Interior}

U.S. Geological Survey

'U.S. Geological Survey, Menlo Park, Calif.

${ }^{2}$ COSMOS Virtual Data Center, University of California, Santa Barbara (now at: NEESit, NEES Cyberinfrastructure Center, University of California, San Diego, La Jolla, Calif.).

${ }^{3}$ California Geological Survey, Office of Strong Motion Studies, Sacramento, Calif.

${ }^{4}$ Kircher and Associates, Palo Alto, Calif.

${ }^{5}$ Wiss, Janney, Elstner Assoc., Inc., Emeryville, Calif. 


\section{U.S. Department of the Interior DIRK KEMPTHORNE, Secretary}

\section{U.S. Geological Survey Mark D. Myers, Director}

U.S. Geological Survey, Reston, Virginia: 2008

For product and ordering information:

World Wide Web: http://www.usgs.gov/pubprod

Telephone: 1-888-ASK-USGS

For more information on the USGS-the Federal source for science about the Earth, its natural and living resources, natural hazards, and the environment:

World Wide Web: http://www.usgs.gov

Telephone: 1-888-ASK-USGS

Suggested citation:

Evans, J.R., Squibb, Melinda, Stephens, C.D., Savage, W.U., Haddadi, Hamid, Kircher, C.A., and Hachem, M.M., 2008, The Virtual Data Center Tagged-Format Tool; introduction and executive summary: U.S. Geological Survey Open-File Report 2008-1327, 171 p. [http://pubs.usgs.gov/of/2008/1327/].

Any use of trade, product, or firm names is for descriptive purposes only and does not imply endorsement by the U.S. Government.

Although this report is in the public domain, permission must be secured from the individual copyright owners to reproduce any copyrighted material contained within this report. 


\section{The Virtual Data Center Tagged-Format Tool: Introduction and Executive Summary}

By John R. Evans, Melinda Squibb, Christopher D. Stephens, W.U. Savage, Hamid Haddadi, Charles A. Kircher, and Mahmoud M. Hachem

\section{Executive Summary}

This Report introduces and summarizes the new Virtual Data Center (VDC) Tagged Format (VTF) Tool, which was developed by a diverse group of seismologists, earthquake engineers, and information technology professionals for internal use by the COSMOS VDC and other interested parties for the exchange, archiving, and analysis of earthquake strong-ground-motion data.

\section{Background}

For many decades, specialized formats have been defined and used to encode and store digitized acceleration time-series data from strong-motion sensors, as well as information derived from these data (for example, the "response spectra" widely used by structural engineers for design and retrofit of structures subject to seismic hazards). While many strong-motion formats currently in use derive in some way from the California Institute of Technology Blue Book format (circa 1970), there is little accepted standardization. Additionally, as compared to other seismic disciplines, the strong-motion and engineering communities require much more extensive supporting and derivative data to adequately qualify a record; thus, the use of more modern formats, such as SEED and SAC, is precluded.

In many ways current strong-motion formats reflect an early period of digital computation and storage when such resources were limited by today's standards. These early facilities also relied on the column-formatted "flat files" used by Fortran and lacked modern information-technology (IT) notions, such as "structured" storage, "object-oriented" and "platform-independent" programming, markup languages like xml, and relational databases.

The COSMOS v1.20 fixed-field format successfully amalgamates the various derivatives of the "Blue Book" fixed-field format and other compatible formats and could be used as a standard mechanism for exchanging data among various institutions and individuals worldwide; indeed, it is being used in the exchange of data between several institutions within California (the California Integrated Seismic Network), for example.

There is an opportunity and a need for mechanisms of strong-motion formatting, data exchange, and processing that exploit modern resources and techniques. There is also an opportunity to create a viable international exchange and format-conversion medium - strong-motion seismology and earthquake engineering depend upon international exchange of data and results. For example, the data provided to the world by Taiwan's Central Weather Bureau for the Chi-Chi earthquake mainshock more than doubled the worldwide strong-motion database and addressed issues that were previously 
untenable. In particular, the global scope of VDC activities necessitated a medium of conversion between the numerous extant, historical, and likely future formats for strong-motion data and products. This VTF tool is intended to fill this need. By analogy to telephony switching centers, the VTF tool will be used as a crossbar switch, interconnected and compatible with all sources and users of strong-motion data. The VTF, and specifically its xml mirror, currently serve as a primary data-storage, data-exchange, and operational tool within the VDC IT structure.

The limitations inherent to fixed-field formats and legacy IT techniques motivated our indepth review of strong-motion formatting, data exchange, operations, and management, resulting in this proposed VTF along with its exactly equivalent $\mathrm{xml}$ representation and provision of key user tools for both the VDC and other interested parties.

\section{Approach}

The VTF tool is designed with the following key goals and constraints:

1. To be readily and unambiguously readable throughout by both humans and computers;

2. To be expressly international in its viability, including support of non-English scripts and the use of English - the current international standard for science and engineering - as its basis;

3. To be inherently both "backward compatible" with older versions of the VTF and prospectively extensible with minimal effort by the VDC and all potential users (that is, no tag will ever be dropped from subsequent versions, so that software that can read a later version of the VTF will also be able to read legacy VTF files);

4. To be inherently self-documenting and autonomous (that is, not requiring reference to any external information for the meaning of all parts of a record to be clear to future users even when all supporting information, such as this document, is lost; and

5. To be easily translated to and from both fixed-field-formats and formally structured formats, including xml, Excel ${ }^{\mathrm{TM}}$, relational databases, and other media now in use or likely to evolve.

To a lesser degree, the VTF tool also is designed to minimize redundancy and duplication within each file - that is, to express a given piece of information in exactly one location. Because of widely reported difficulty with parsing multiple-record files, we also limit VTF records to a single component of data per file. Finally, the notion of separate text, real, and integer header sections is supplanted by a universal form of data-type identifier, allowing either alphabetical or logical arrangements of the information for ease of use and for making evident the relationships between tags. The notions of data types remains, but only as guidance incorporated directly in the name of each variable (in the formal IT sense, all VTF data are text, including the text representations of numbers).

To accomplish these goals, we have chosen a simple tagged format exemplified by

Descriptive.Tag_type = value units;. Within this tagged format we also implement a modern "structured" or "class" design that is easily understood by a reader but readily translated by computers to and from other structured forms. Structuring the tag names also makes relationships between them more evident. Thus, we have made every tag name, its value, and its units of measure explicitly descriptive (and have provided for extensive comments). This design is the essence of dual human- and computer-readability, as well as of extensibility and autonomy. 
An example of one minor set of entries for a VTF file is:

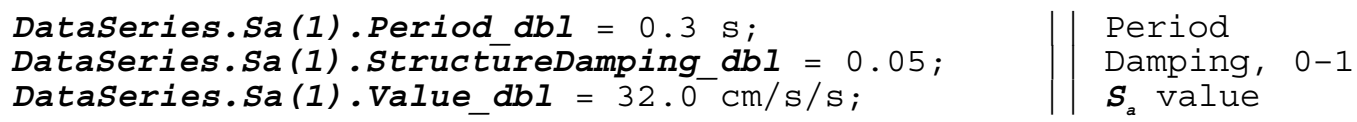

This example defines the response spectral acceleration $\left(32 \mathrm{~cm} / \mathrm{s}^{2}\right)$ at a period of $0.3 \mathrm{~s}$ for 5 percent structure damping (0.05 fractional damping). The comments at the right or in the numerous Comments tags are anything the users believe will be helpful to themselves or others. The index (1) illustrates that arrays of tags - arrays of any length — can be created.

Three departures from Blue-Book-style formats are:

1. There is no longer either a fixed number or any concatenation of real, integer, or text variables (in order to allow greater efficiency and extensibility as well as more convenient or logical organization);

2. There are no coded entries, that is, no look-up tables requiring the survival of those tables for the entry to be meaningful. Instead, all table values are clearly understandable words, phrases, or values ( \pm units) whose meaning will be the same in thirty years as they are today without reference to the tables from which they come; and

3. We have corrected the few typographical errors and omissions in the v1.20 fixed-field format while the opportunity for complete, detailed descriptions also is greatly expanded.

Where look-up tables are used, in addition to the listed self-evident values most such tables are extensible. That is, VTF users may define new entries if they do not find a suitable value already listed, so long as it is terse and clear to all users. Similarly, users may invoke Private tags when no extant tag suffices. While it is highly desirable to use standardized values and tags, and we have attempted to be comprehensive, these facilities accommodate items we have not anticipated and provide a means for suggesting additions to the VTF, which will be reviewed and acted upon.

\section{Utility}

Applications of the VTF include providing a generalized storage and translation medium for use by the VDC to simplify and expedite translations between all sources and users of strong-motion data internationally. The VTF tool is available for download and use by any interested party and is complemented by a series of portable software applications designed to make implementation straightforward.

Because we have kept the essence of the COSMOS v1.20 fixed-field format in the VTF tool, all of the Blue Book data dictionary is reproduced here, though sometimes rearranged or generalized. Thus, it is straightforward to translate unambiguously between VTF and the v1.20 fixed-field format and, by extension, all other formats.

The support tools currently available from the VDC are self-updating Java ${ }^{\mathrm{TM}}$ routines for access, display, and input/output; these subroutines can become the front and back ends of existing processing systems as desired. Initially, we are supporting Fortran, C, MatLab ${ }^{\mathrm{TM}}$, and Excel ${ }^{\mathrm{TM}}$, which are used widely by these communities. We also ask the strong-motion engineering and seismology community for consensus on other software worthy of direct support. By design, the translation routines are easily extended to other formats, thus are the basis of a universal translator at the VDC. 


\section{Appendix}

Attached at the following page is the current governing document for the VTF. Although this document is detailed and in some ways difficult to read, it is the sole formal definition of the format and is kept up-to-date and distributed only by the VDC and its assigns. The most current version can be downloaded at http://db.cosmos-eq.org/FormatConversion.html if they postdate this Open-file Report. 


\title{
The COSMOS Virtual Data Center Tagged-Format Tool: Formal Definition [Version VTF.1.0]
}

\section{(16 October 2008)}

\author{
John R. Evans ${ }^{1}$, Melinda Squibb ${ }^{2}$, Christopher D. Stephens ${ }^{1}$, W.U. Savage ${ }^{1}$, \\ Hamid Haddadi ${ }^{3}$, Charles A. Kircher ${ }^{4}$, and Mahmoud M. Hachem ${ }^{5}$ \\ ${ }^{1}$ U.S. Geological Survey, Menlo Park, Calif. \\ ${ }^{2}$ SOPAC, University of California, San Diego, La Jolla, Calif. \\ ${ }^{3}$ California Geological Survey, Office of Strong Motion Studies, Sacramento, Calif. \\ ${ }^{4}$ Kircher and Associates, Palo Alto, Calif. \\ ${ }^{5}$ Wiss, Janney, Elstner Assoc. Inc., Emeryville, Calif.
}

(There is a Table of Contents at the front and an Index of Tags at the end of this document. This is the sole, formal, governing document for this format, and as such is authoritative but difficult reading. The Executive Summary and other supporting items are supplied only for the user's convenience.) 


\section{Table of Contents}

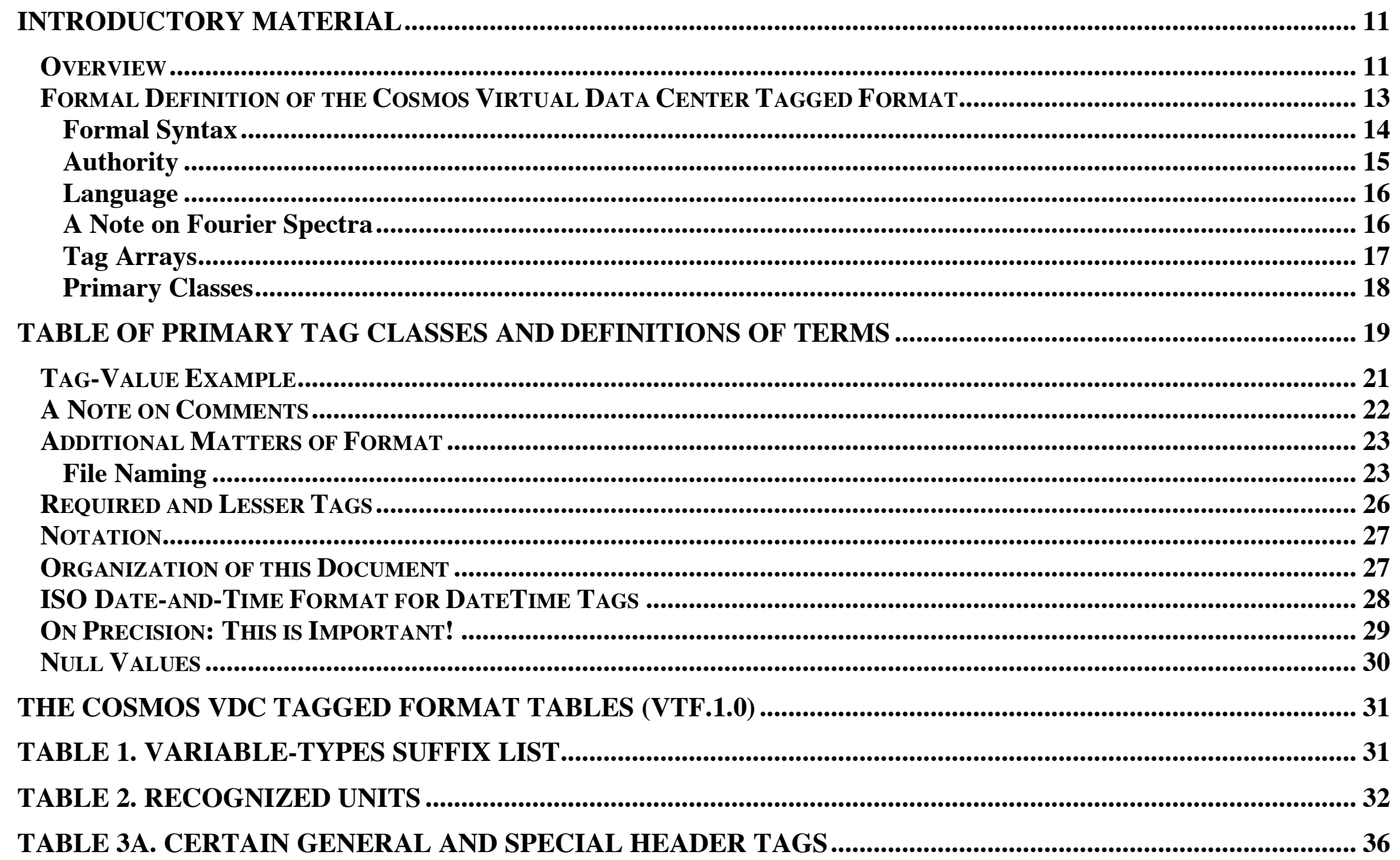


TABLE 3B(A). TYPE OF DATASERIES

TABLE 3B(B). CODES FOR TYPES OF DATASERIES ......................................................................................... 42

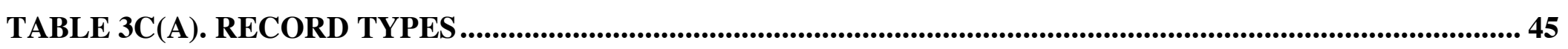

TABLE 3C(B). RECORD-TYPE AND INPUT-TYPE CODES ...........................................................................46

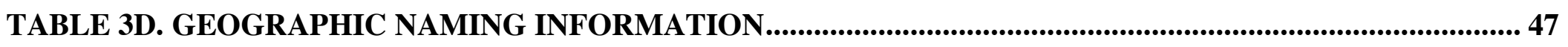

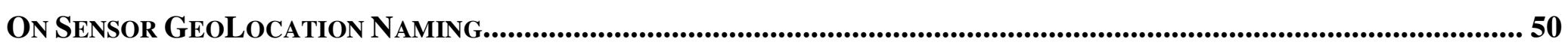

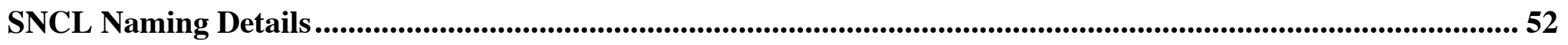

TABLE 3E(A). ARRAY VARIABLES, INCLUDING NETWORK/OWNER/AGENCY CODES ............................ 53

TABLE 3E(B). COSMOS AND FDSN ARRAY/NETWORK CODES ............................................................................. 56

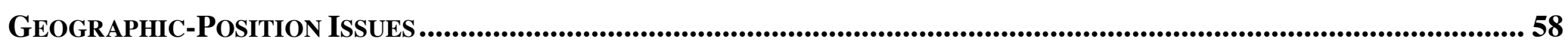

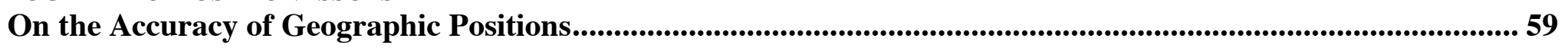

TABLE 3F. OTHER GEOGRAPHIC AND ARRAY TAGS－COORDINATES .............................................59

TABLE 3G. GEODETIC ELEVATION DATUMS............................................................................................64

TABLE 3H(A). SITE TYPE AND THE BUILT ENVIRONMENT .................................................................................66

TABLE 3H(B). SITING CONDITIONS, REFERENCE, AND FREE FIELD ........................................................... 78

TABLE 3H(C). BUILT ENVIRONMENT VALUES (CSMIP) ..................................................................................... 79

TABLE 3H(D). SITING CONDITIONS, BRIDGES .......................................................................................................... 81

TABLE 3H(E). SITE TYPE AND BUILT ENVIRONMENT VALUES - HAZUS CODES ................................. 82

TABLE 3H(F). OCCUPANCY CLASSES - HAZUS CODES .......................................................................... 84

TABLE 3I. EVENT (EARTHQUAKE) INFORMATION .....................................................................................85

TABLE 3J(A). DATA-ACQUISITION UNIT (DAU; RECORDER; DATA LOGGER) INFORMATION ................ 96

TABLE 3J(B). DAU MODELS AND MANUFACTURERS ...................................................................................... 102 
TABLE 3K(A). SENSOR INFORMATION.

TABLE 3K(B). SENSOR MODELS (USE TABLE 3J(B) FOR MANUFACTURERS).

TABLE 3K(C). SENSOR DIRECTION CODES FOR USE WITH SENSOR.ORIENTATION ONLY (AVOID) ... 116

TABLE 3L. DATA SERIES (INCLUDING ORIGINAL)： TIME-SERIES OR SPECTRUM INFORMATION ..... 118

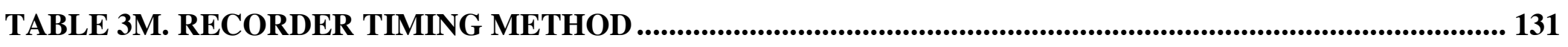

TABLE 3N. PROCESSING INFORMATION.......................................................................................................... 132

TABLE 3O(A). CODES FOR FILTER TYPES USED IN PROCESSING ................................................... 143

TABLE 3O(B). CODES FOR FILTER TYPES USED IN PROCESSING ................................................................... 144

TABLE 3P. RELATIONAL TAGS INVOLVING TWO GEOGRAPHIC LOCATIONS................................... 145

TABLE 3Q. PRIVATE TAGS (AVOID) ........................................................................................................... 147

TABLE 3R. DATASERIES TAGS CLOSELY ASSOCIATED WITH TABLE 4 ............................................ 149

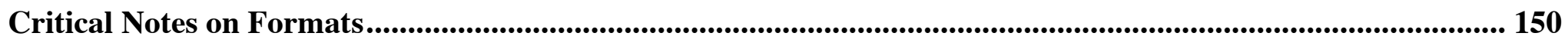

TABLE 4. THE TIME SERIES OR SPECTRUM (THE DATASERIES VALUES) ............................................... 154

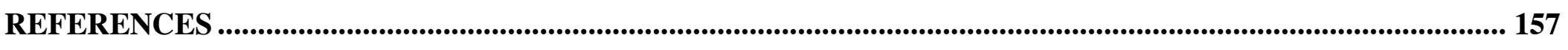

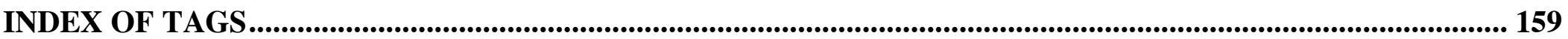




\section{Introductory Material}

\section{Overview}

The COSMOS v1.20 fixed-field format was an effort to unite in a single forum the various derivatives of the CalTech "Blue Book" formats being used to store and distribute strong-motion data. Issues of readability, generality, comprehensiveness, structure, and autonomy motivated revisiting the format issue in depth, resulting in this proposed COSMOS VDC Tagged Format (VTF) and its equivalent xml mirror as additional tools for the VDC (Virtual Data Center) and others who may find these tools useful. Efforts are underway to revise the fixed-field format, as well.

Primarily, the VTF tool is designed to be readily and unambiguously readable throughout by both humans and computers, to be expressly international in its viability, to be inherently both backward compatible and extensible with little effort (thus, easily maintained), to be inherently self documenting and autonomous (that is, not requiring reference to any external information for the meaning of all parts to be clear in the future even when all supporting information, including this document, are lost), and to be translated easily to and from structured formats, including xml, Excel ${ }^{\mathrm{TM}}$, databases, and other common media. To a lesser degree, this format tool also is designed to minimize redundancy and duplication in each file (to express a given piece of information in one location only, feasible because of its dual human and computer readability). The notion on text, real, and integer header sections is supplanted by a universal form of header information.

The tagged format's applications include providing a generalized storage and translation medium for the COSMOS VDC, a medium that can absorb and translate data from all sources internationally. Thus, the format is intended to be a superset of all formats now used in strong-motion work and to anticipate likely future needs. The tagged format also is available for use by any interested party, along with a series of tools designed to make that use straightforward.

By "backward compatible" we mean that parsing (reading) software for VTF Version VTF.1.1 and beyond will be able to read earlier VTF versions without difficulty, and this functionality will persist as a natural part of the format definition. 
To accomplish the goals enumerated above, we have chosen a simple "tagged" format (and within this tagged format a modern "structured" or "class" design that will be both user friendly and still readily translatable by computers to and from other formats).

We have made every tag name (and its value) explicitly descriptive and have added extensive comments in this governing document about each tag's purpose and rules of use. Indeed, most of these tag names should look familiar to those who know the Blue Book formats and, where not so, we have commented about what equivalent variables our tags replace.

This design is the essence of dual human- and computer-readability. Everything expressed in VTF is made easy to read, understand, and bring into analysis systems.

Two departures from the Blue Book formats are, first, that there is no longer a fixed number of real or integer variables; this allows both greater efficiency and extensibility. Second, there are no longer any "coded" entries-no references to tables of entry definitions that must survive into posterity. Where there are tables of values that can be entered, all the values in those tables are clearly understandable words, phrases, or values whose meanings will remain the same and will not require reference to the tables from which they come.

In all instances we have tried to keep the essence of the v1.20 fixed-field format alive and well in the VTF tool. Although rearranged and recast into a more parseable form, all of the Blue Book data dictionary is reproduced here in some form. It is, therefore, straightforward to translate unambiguously between VTF and any of these Blue-Book formats. The VDC will be providing translation matrices and Java translation facilities, as well as subroutines for parsing and for writing VTF; these subroutines can become the front and back ends of existing Processing systems if desired. We will provide these front- and back-end routines in Fortran, C, MatLab ${ }^{\mathrm{TM}}$, and Excel ${ }^{\mathrm{TM}}$, and will ask the COSMOS community for consensus on other widely used software. The general translation routine will be in (automatically selfupdating) $\mathrm{Java}^{\mathrm{TM}}$ and driven by Excel ${ }^{\mathrm{TM}}$ tables describing the various formats. Thus, the translation routine is easily extended to additional formats and we anticipate supporting all widely used formats. Similarly, VTF can be used as the core of a "universal translator" connecting any of the formats described in those Excel" ${ }^{\mathrm{TM}}$ tables. This translator and the Java ${ }^{\mathrm{TM}}$ interface to VTF will be key tools for implementing, refining, and extending COSMOS VDC functionality and user friendliness. 
Finally, we have added items not available in v1.20 of the fixed-field format, such as various new types of instruments and manufacturers from Japan and Germany, Laplace, and z-plane descriptions of instrument and filter responses and to correct a number of trivial typographical errors.

The version of this document released by the COSMOS VDC is the sole formal definition of the COSMOS VDC Tagged Format Tool (VTF). Therefore, no change to the format or its use-rules from any other source is permitted. This document is long and detailed; a more easily understood executive summary and access tools for the format are available from the COSMOS VDC.

\section{Formal Definition of the Cosmos Virtual Data Center Tagged Format}

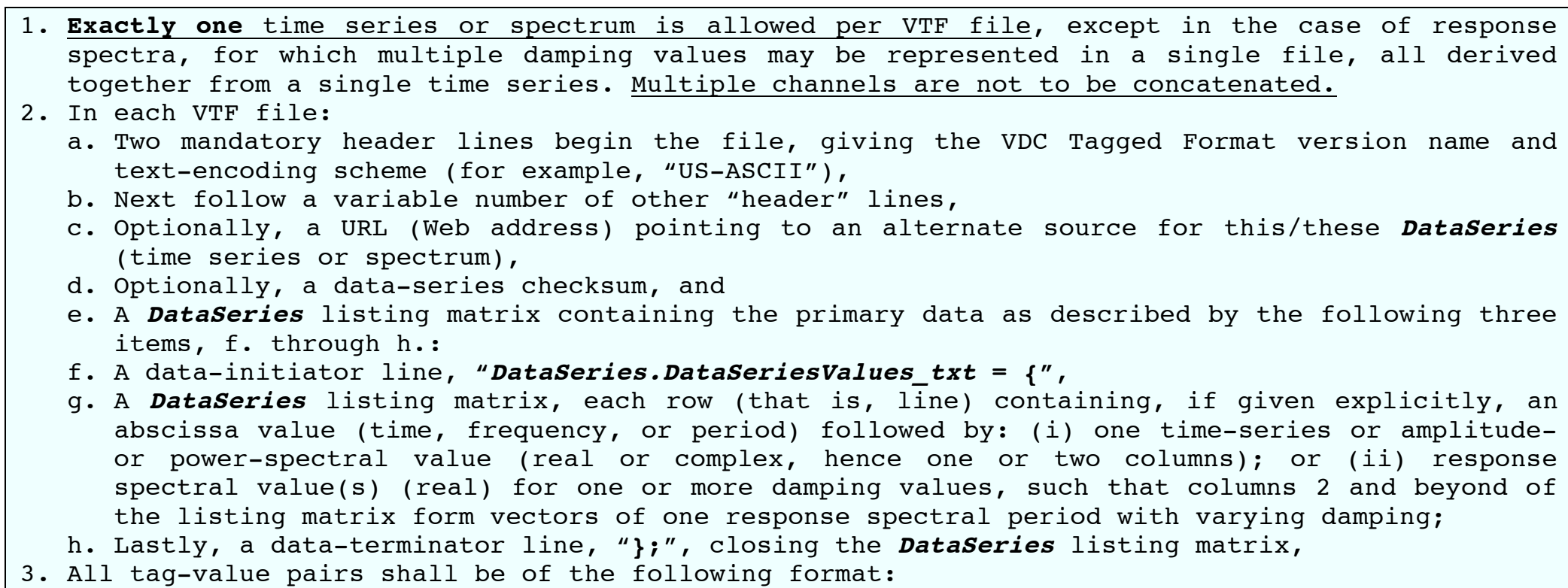




\section{Formal Syntax}

<TagName>_VVariableType> = <value>[ <units>]; [ <white space>] | <comments>]
or
[<white space>] | | <comments>

where TagName is defined by

$<$ TagName $>=<$ ClassName $>[(\mathrm{m})][$. $<$ SubClassName $>[(\mathrm{n})][.<$ SubSubClassName $>[(0)] \ldots]]<$ MemberName $>$

As is common in software documentation, " $\diamond$ " delineates a description of what belongs there while "[]" delineates optional or occasional elements, possibly nested. Note that <TagName>_<VariableType> (as well as any text values taken from the tables below) are case sensitive. Tag values are usually in English; tag names are always in English.

Similarly, neither tag names nor text values may contain any of the following characters: tabs, ", ' (double and single quotes), and ' (grave accent). Such characters can be misunderstood by xml and databases.

Furthermore, while tag names shall not contain white space characters, there shall be white space on both sides of the equal sign (" =") and between the <value> and any <units>. White space may be blanks or tabs. Finally, note the presence of the semicolon after either the <units $>$ or a unitless <value $>$ - that is, the semicolon terminates the "tag = value [units]" sequence and must be present.

White space also is allowed almost anywhere else, but not within tag names nor within numerical values (you can put white space inside text that you create yourself, but you must not inject it into text values taken from tables-use those exactly as they appear in the tables).

Numerical values may appear in integer, floating point, or scientific notation, but must be in base ten. (To avoid decimal-binary-decimal translations, thus preserving accuracy, numerical values are treated as text by most VTF utilities until such time that they must be operated upon as numbers, such as for plotting the DataSeries.) 
In most cases tags can be given in any order, however, it is recommended that either an alphabetic order or the functionally organized order shown here generally be respected so that tags can be located easily. The Index of Tags near the end of this document is a comprehensive alphabetical list that may aid users.

(There are three exceptions to the flexible-order rule, one at the top and two at the bottom of the file. (1) The first tag in table 3A must appear first in the file, (2) the tags surrounding the DataSeries are in a specific order, and (3) the DataSeries listing matrix, when the abscissa is implicit, must be in increasing order of the abscissa's value. We encourage the same practice even when the abscissa is given explicitly as the first (or first two) columns of the DataSeries listing matrix.

As implied above, there are instances in which the abscissa of a time series or spectrum must be given explicitly as the first one (real) or two (complex) columns of the DataSeries listing matrix. However, there are many common situations in which is it sufficient to define the abscissa implicitly, as with regularly sampled time series and Discrete Fourier Transform (DFT) spectra. Many other spectral forms will require an explicit spectrum. The rule is that if an abscissa can be correctly defined by a starting point and an increment, it may be (but does not have to be) given explicitly. All other cases must provide an explicit abscissa. Instances of requisite explicit abscissas commonly include response spectra and power spectra computed from a DFT that is spaced evenly in frequency but where the power spectrum is provided as a function of period.

\section{Authority}

The only authoritative definition of the COSMOS VDC Tagged Format (VTF) is this document, as maintained by the COSMOS Virtual Data Center or its assign.

Nevertheless, the VTF has many "user-extensible lists" (tables of values) for which a user may assign any reasonable value, as long as it starts with the string "User's description:" (to help us make format-verification work well in the most general case). Such user-created values in extensible lists are likely to help us expand this authoritative VDC document. Similarly, use of Private tag sets, defined in table 3Q, also may inform our modifications of the VTF.

Private tag sets and user-created text values should be limited to necessity and kept reasonable and likely to be understandable to any user at any future time. 


\section{Language}

The official language of all tags (including Private tags) and of most text values is English. However, we recognize that certain text values, such as the descriptive name of a geographic location (GeoLocation.Name.Description) and addresses (GeoLocation.Name.Address), sometimes must be in their local languages to be meaningful. Furthermore, translation of comments and other errata into English should not be allowed to become a barrier to the dissemination of data.

\section{A Note on Fourier Spectra}

For a DFT, if only the non-negative frequencies for a real time series are to be given in this VTF file, then the power in the negative-frequency bins generally must be added to their corresponding positive-frequency bins so that the positivefrequency bins represent the total power at each frequency (generating a true one-sided spectrum). For real time series, this correction commonly can be accomplished by scaling up the non-Nyquist positive-frequency bins by the square root of two, however, caution is appropriate.

(Many FFT implementations of the DFT yield their output in some order or another, such that it contains negativefrequency bins, one 0-Hz ("DC") bin, positive-frequency bins, and one Nyquist bin. Typically then, half the power of non-Nyquist finite-frequency bins is to be found in the negative-frequency bins. Thus all non-Nyquist positive-frequency bins are likely to be too small by a factor of the square root of two. In contrast, most of these FFT routines put all the power of the $0-\mathrm{Hz}$ and Nyquist bins each into a single bin, so that these two bins do not need correction.)

Since many FFT routines differ in their output formats and rules, it is essential that the user test the behavior of their FFT routine with real data to determine whether this correction for power lost to negative-frequency bins is required as well as to determine the particular behavior of the $0-\mathrm{Hz}$ and Nyquist bins.

To test an FFT routine, take the FFT of one or more pure sine waves fitting exactly into the input window and using no weighting in that window (that is, a boxcar window). "Fitting exactly" means that the time-series point just after the end of the input window and, therefore, not present in the data provided to the FFT, must exactly equal the first point in the input window and be in the same phase. That is, that the window duration is one sample shorter than a full integer 
number of cycles. This arrangement is called "DFT symmetry"-because the DFT assumes a precisely periodic input, repeating the first point just after the last and so forth, DFT symmetry yields an effectively infinite-duration pure sine wave which will put all its power into exactly one frequency bin or exactly two complementary-frequency bins, positive and negative - there will be no side lobes. The total power contained in either the one bin or the positive-negative pair of bins will equal the power computed in the time domain. If a pair of bins is required to match the total power in the time domain, then the correction to non-Nyquist positive-frequency bins is required when providing only the non-negative bins in the VTF file. Similarly, it is possible to verify whether all or half the power of the $0-\mathrm{Hz}$ or Nyquist bins is in one bin.

\section{Tag Arrays}

The VTF allows Subscripted arrays of variables (that is, arrays of tags). This facility allows for things like enumerations of filter poles and zeros, several earthquakes appearing in a single record (for example, rapid-fire aftershocks or swarm earthquakes), and historical information about an instrument.

These "tag arrays" are implemented by the " $(\boldsymbol{m})$ " part that is shown at the ends of class or subclass names as indicated in this document - in other words, Subscripts are not allowed for a tag or portion of a tag unless shown in this document. Subscripts must be positive integers $(1,2,3, \ldots)$ with no missing values, so they are in the MatLab ${ }^{\mathrm{TM}}$ or Fortran style. Subscripts are not in the C style of non-negative Subscripting $(0,1,2, \ldots)$.

For example if a user wants to declare several possible versions of an Event location those tags would be Event.Hypocenter $(\boldsymbol{n})$. In contrast, locations for multiple Events appearing within a single record would be rendered as Event(m). However, it would not make any sense to write Event.Hypocenter.Agency(n), which would imply one Event location with several authors, so this Subscripting is not permitted.

In another example, a user can document multiple instances of names for a spot on the Earth or in a structure (a GeoLocation) - a history of changing names or differing names used by different Agencies for the same instrument. A user might, for example, want to document some name multiplicity with GeoLocation.Name(1).ShortName, GeoLocation.Name(2).ShortName, and so forth, in cases where the Sensor never moved or moved only slightly in the past and is functionally at the same location. GeoLocation(n) makes no sense since in the above example as it would imply a Sensor is in several places at once; this usage is disallowed. 
Classes and subclasses end with periods while class members end with an underscore (“_”). The "(m)" is just before that period or underscore character and just after the class or member name, as in "Sa(2)." or "ResponseSpectrumDamping(3)".

To limit ambiguity, users are urged to keep these Subscripts time-, frequency- or period-sequential where those concepts are meaningful. Note the prevalence of explicit time stamps in this format, which also facilitate documenting historical associations.

Our Subscripting guidance is either " $[(n)]$ ", meaning "optional Subscript here", or "(n)" meaning "required Subscript here". As mentioned, Subscripts may not appear anywhere else. If a tag allows Subscripts but none are given, it defaults to an implicit Subscript of "(1)" - note that only the last of repeated unSubscripted tags will be saved.

\section{Primary Classes}

Both the physical grouping of tags and their < $<$ Sub]ClassName $>$ and <MemberName $>$ are attempts to associate variables in a logical manner. For example, variables having to do with the Data Acquisition Units (DAUs, which are recorders and data loggers) are named by the $\boldsymbol{D A} \boldsymbol{U}$ class; tags associated with the geographic locations ("station names", loosely speaking) are GeoLocation.Name; and tags associated with the source event are Event. The following table includes a complete list of the primary ("top") set of Classes and their definitions. 


\section{Table of Primary Tag Classes and Definitions of Terms}

\begin{tabular}{|c|c|}
\hline ClassName & Meaning \\
\hline Thisfile & $\begin{array}{l}\text { A small set of special tags which apply to this VTF file, such as those } \\
\text { naming the version of the format and the VDC's unique identifier of this } \\
\text { record. }\end{array}$ \\
\hline Array & $\begin{array}{l}\text { Any set of Sensors plus DAUs at one or more Geolocations that is managed as } \\
\text { or logically forms a single data-acquisition system-for example, a } \\
\text { structural Array, a geotechnical Array, or a single free-field instrument } \\
\text { at a single GeoLocation. (An Array with a single-Geolocation is the usual } \\
\text { case in the free field, but it is still an Array in the same sense that a } \\
\text { scalar is a one-element vector). } \\
\text { Note that arrangements many of us think of as and call "an array" (such as } \\
\text { the NESMP "array"), are not Arrays as narrowly defined here. In the VTF, } \\
\text { such agglomerations are more properly called an Agency.t }\end{array}$ \\
\hline GeoLocation & $\begin{array}{l}\text { The exact geographic location in three dimensions of the point of } \\
\text { measurement (strictly speaking, of the Sensor's proof mass). } \\
\text { Note that this is a very narrow definition and defines the location of a } \\
\text { single sensor or package of several axes. Even some classical simple } \\
\text { "stations" with sensors spaced a few meters apart might be considered by } \\
\text { some users to occupy several distinct Geolocations, for example, in cases } \\
\text { of precise GPS location and large ground-motion spatial variance. } \\
\text { We strongly discourage the practice of giving the same set of coordinates } \\
\text { for all members of an Array, such as the Array monitoring a dam site. This } \\
\text { practice obviates many applications of the data.t }\end{array}$ \\
\hline GeoLocationRupture & Tags relating GeoLocations to fault-rupture surfaces. \\
\hline Event & $\begin{array}{l}\text { The signal of interest (for example, the natural or artificial source of } \\
\text { ground motion, a calibration signal, and so forth). }\end{array}$ \\
\hline EventGeolocation & Tags relating Events to the Geolocations of Sensors. \\
\hline
\end{tabular}




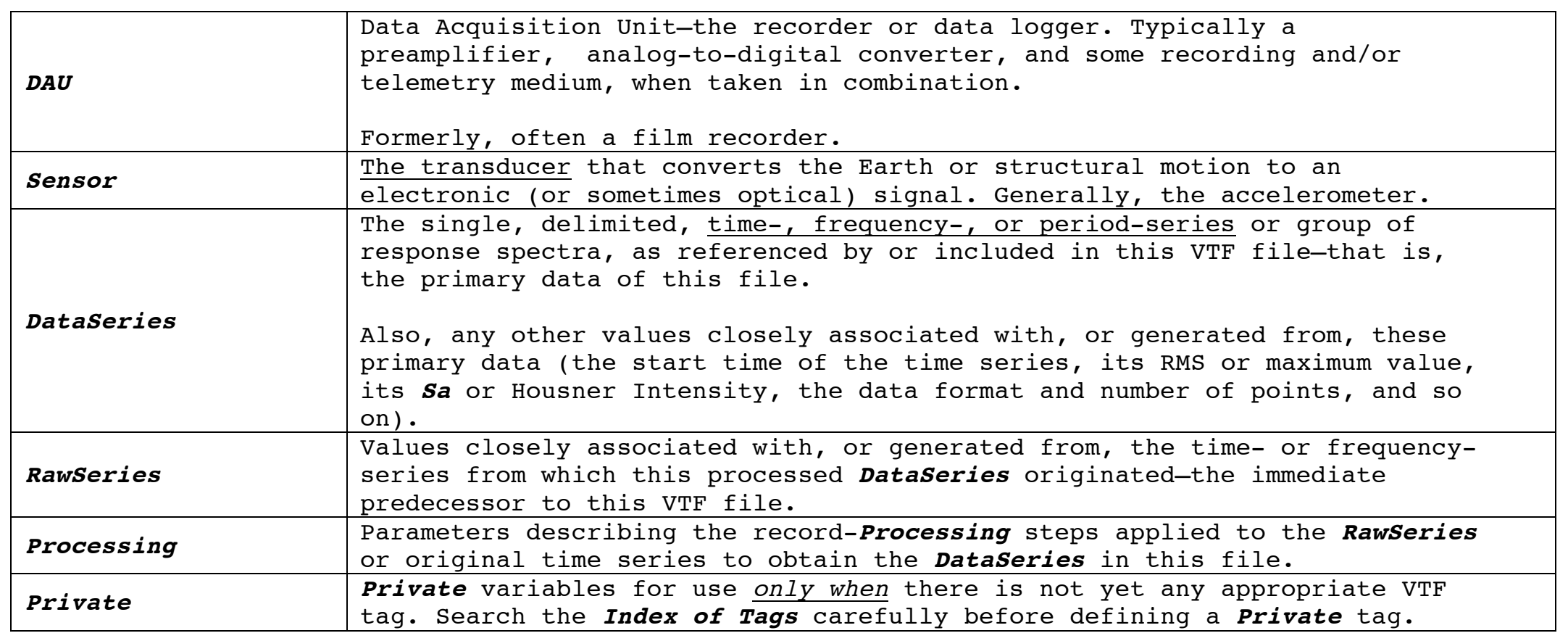

$\dagger$ The reader may notice that we rarely use the word "station", instead nearly always using either the word Array or the word GeoLocation as they are narrowly defined above. We do so because the word "station" means many different things to different practitioners, in some cases signifying an entire Array. Similarly, we limit our use of the word "Site" to mean the site conditions at a particular GeoLocation; "site" has other special meanings to large portions of the COSMOS community, as in "work site", which is broader then our definition (notwithstanding that many GeoLocation.Site data are generalized for an entire work site, often for reasons of cost, and not as specific to a particular GeoLocation as one might desire.) 
In tags identifying entities responsible for various parts of this VTF file (such as the owner/operator of the instrument in question) we use the word "Agency". By selecting this word, we do not mean to limit in any way the type of entity (organization or individual) that may be named in such variables. The term Agency is nothing more than a reasonably general <MemberName> with a meaning tolerably close to its intended use. We expect that many an "Agency" will be an individual person (such as amateurs of the Public Seismic Network), a secondary-school classroom, a Government agency, a consortium such as IRIS, or a college or university (this name is analogous to a GVDC "Business Associate").

In all cases, we intend that preference be given to identifying Agencies by FDSN network names, some of which are shown in the second column of table 3E; additional names that may be used are given in the first column of that table. If no appropriate Agency name is available from these lists, the user may insert some terse, well known, identifiable abbreviation for an organization's name or insert the individual's name. That is, the Agency list is "user extensible"-it is unlikely that the we have been comprehensive and we do not wish to exclude any Agency from the VTF.

\section{Tag-Value Example}

To illustrate this format, the following lines represent typical spectral acceleration values at the two periods, 0.3 and $1.0 \mathrm{~s}$, for 5 percent damping:

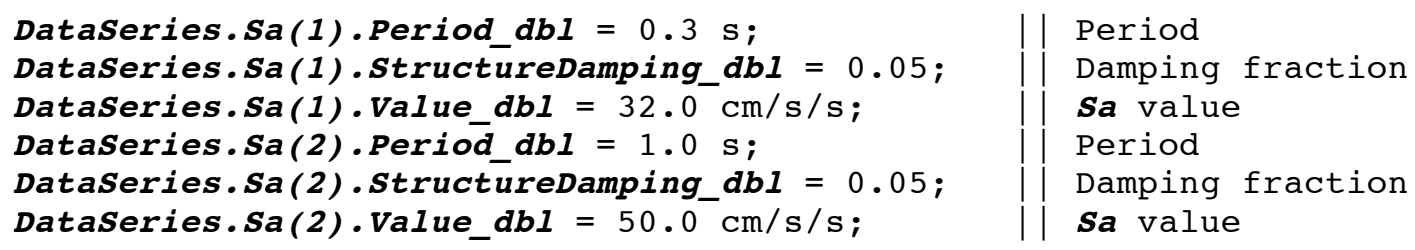




\section{A Note on Comments}

There are two kinds of comments in the VTF: (1) comments associated with a particular group of tags (any Comments, Description, Annotations, or Citation tags) and (2) comments that can be put almost anywhere, but which are given limited treatment by the parser (for example, "Il This value needs updating.").

For all important comments, please give preference to the group of tags ending with Comments, particularly those that you wish to associate rigorously with a particular subject matter. Although "Il" comments are brought forward by the parser, they are associated only loosely with their original subject (by position in the VTF file). For example, in the xml mirror of the VTF "Il" comments are added to the item at which they are given as a "Note" xml type. That is, the parser simply attaches all "Il" comments to the nearest tag above the "Il" comment). Thus, "Il" comments are primarily for human consumption and not computer manipulation and should be avoided.

Nevertheless, "Il" comments are used extensively in this governing document and other supporting materials to elucidate details of usage - these comments generally should not be included in any working file.

In practice, "I|" comments might be used to provide temporary notes by the user to the user (for example, "Il still need to fix this") or to make minor comments that do not need to be associated rigorously with any particular tag. However, "Il" comments should not be used to make comments that are important in any significant way to the data.

Note that this is a change from the similar looking comments of the Blue Book formats. Important comments should be put into the variable ThisFile.Annotations.TextValue if not elsewhere associated with specific portions of the header in one of the other Comments (or Description, Annotations, or Citation tags) variables.

(The xml class Note is not visible to the user. It is used by parsers to store "Il" comments in proximal association with the tags to which they are appended. All such comments are attached to the nearest previously encountered tag.) 


\section{Additional Matters of Format}

Blank lines and lines consisting entirely of comments may appear anywhere after the first line excepting within the DataSeries.DataSeriesValues_txt $=\{\ldots\}$; sequence of lines. Studies have shown that simply inserting blank lines in appropriate locations, such as between major header sections and between logically associated blocks of information, greatly improves the human readability of files. We commend such uses of white space to the reader and make extensive use of it in this governing document.

There is no firm limit on line length since the COSMOS community is no longer bound by the rigors of punched cards. Nevertheless, for the sake of keeping files easy to read on modern workstation screens, users are urged to keep line lengths below 100 characters where feasible. This length easily fits onto screens possessed of moderate resolution at reasonable character size without imposing unreasonable limits on the sizes of tags or their values. (This document uses about a 100-character line length, for example.)

The primary exception to this voluntary constraint is for Comments, Description, Annotations, and Citation tags (the preferred sorts of comments), which users may wish to make long in many cases. A word-based wrap-around display rule in your editor of choice should be sufficient to ensure readability of such text, as long as users are reasonably careful to write with adequate white space inside the comment text.

In particular, please replace line break characters with the four-character sequence " $\mathrm{n} \boldsymbol{} \boldsymbol{}$ ' to ease the reader's job. (The " $\boldsymbol{n}$ " is the C-language symbol for "newline".)

In any case, lines shall not be blank padded on the right. (All lines will be read in as strings by the parsers, so blank padding simply wastes file space.)

\section{File Naming}

We suggest, but do not require, a format such as the following for naming VTF files:

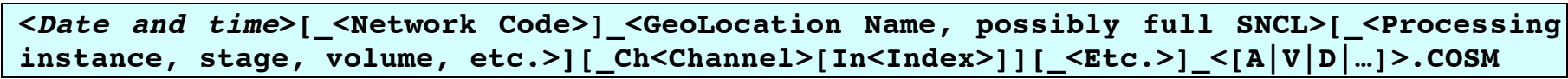


The COSMOS VDC will use such filenames in data it translates into VTF for a user.

For example, "20021103_221245_XX_PS09_Op1_Vo2_Ch2_V.COSM" is a record from the Denali fault earthquake of 03 November 2002 ("20021103_221245") for Alyeska ("_XX" == "other network") station [GeoLocation] "PS09", evaluated with "Processing option/instance" one ("OOp1"), fully processed ("Vo2" == Volume 2), for Channel 2 (“_Ch2"), and is a velocity trace (“_V”). The "COSM" (or "cosm") means "a COSMos vdc tagged format".

The <Date and time $>$ stamp should be in largest-to-smallest order and generally should be to the nearest second; we suggest the format YYYYMMDD_hhmmss, as in "20051117_173802”, which is a variant of the ISO time format we use here.

Note that this $<$ Date and time $>$ should be the best available time, preferably the time of the first sample. If that instant is not known, use the trigger time of that record. If even that is not known, use the origin time of the Event. (This ordered list of options is for the sake of consistency between records and to take account of legacy data, which often have very poorly known timing.)

The <Network Code> is from either Array.Agency or the "NN" field in GeoLocation.Name.SNCL or GeoLocation.Name.ExpandedSNCL.

The <GeoLocation Name, possibly full SNCL> is whichever of GeoLocation.Name.SNCL, GeoLocation.Name.ExpandedSNCL, or GeoLocation.Name.ShortName is used to identify the GeoLocation of this Sensor, with a SNCL name preferred if several are given. Obviously, use the GeoLocation.Name that is in force at the time of the Event and as used by the Agency providing this record.

The portion "[_Ch $<$ Channel $>[$ In<Index $>]$ ]" comes from the tags Sensor.ArrayChannel or Sensor.DAUchannel (giving preference to the former) and from GeoLocation.Name.Index.

In any case, the combination "_[ $<$ Network Code $>]_{-}<$GeoLocation Name $>$... [_Ch $<$Channel $>[$In $<$Index $\left.>]\right]$" should be as detailed as is required to identify uniquely the signals from a specific proof mass (both within that particular Array and among all your international colleagues). That is why a full SNCL name is preferred in place of the entire sequence of strings - the FDSN designed SNCL network names to be unique worldwide. 
The portion "_<[A|VIDI...] $>$ " should always be present and is a string of from one to three letters indicating the type of trace, "_A" for acceleration, "_V" for velocity, "_D" for displacement, and so forth as shown in column two of table $3 \mathrm{~B}(\mathrm{~b})$.

The optional "[_<Processing instance, stage, volume, etc.> $]$ " identifies the stage of Processing, for example "_Vo2" for the equivalent of a Blue Book "Volume 2" record. This portion of the VTF filename should correspond to the meaning of one or more of the following five tags from table $3 \mathrm{~N}$ as shown here:

\begin{tabular}{|c|c|}
\hline Tag and value & $\begin{array}{l}\text { Filename } \\
\text { fragment }\end{array}$ \\
\hline Processing.BlueBookVolume_int $=[0-3]$; & $"$ "Vo $[0|1| 2 \mid 3] "$ \\
\hline Processing.Stage_txt $=[$ "Raw" $\mid$ "Preliminary" $\mid$ "Final" ]; & “_St $[\mathrm{R}|\mathrm{P}| \mathrm{F}] "$ \\
\hline Processing.HumanReview_txt $=[$ "None" | "Reviewed" | "Updated" ]; & "_Hn $[\mathrm{N}|\mathrm{R}| \mathrm{M}] "$ \\
\hline Processing.Instance_int $=$ <non-negative value $>$; & "_Op<Value>" \\
\hline Processing.SpecialProcessing_txt $=$ " $<$ Value $>$ "; & "SP<Value>" \\
\hline
\end{tabular}

These substrings should be concatenated as needed, as in the example above of "_Op1_Vo2”.

Finally, some users may desire additional differentiating codes, the "[_<Etc.>]".

When filenames begin in this manner with a $<$ Date and time $>$, followed by a string that uniquely identifies the DataSeries, and end with a string that uniquely identifies the data-Processing stage, the resulting files generally will sort easily into Events with the help of typical system file-name-sorting and file-listing algorithms. It also will be clear to colleagues what the name means. Therefore, the portions of the filename after the $<$ Date and time $>$ should as specific as is required for the particular Array and Agency. Ideally, a filename should include a full SNCL name and strings identifying the type of DataSeries and data-Processing leading to the file. As long as an FDSN network code (Agency) appears in the name, there should be no naming conflicts with other Arrays. 
Another example may make this convention clearer for the case of a SNCL name:

20041115_093415_CH4AW.NC.HNZ.01_V2_A.COSM

identifies a "Volume 2" vertical acceleration ("HNZ") record from a temporary USGS instrument co-located with the CSMIP instrument "Cholame 4AW" near Parkfield. The corresponding CSMIP record might be named

20041115_093414_36412.CE.HNZ.01_V2_A.COSM

in this case using the CSMIP five-digit name for the same GeoLocation. There can never be confusion of a differing DataSeries because of the different FDSN network name "CE".

\section{Required and Lesser Tags}

VTF tags come in four flavors: REQUIRED, CONDITIONALly required, Recommended, and Optional. On rare occasions, you also will encounter Avoid, which include Optional tags that are not recommended for use but are kept for backward compatibility and similar reasons.

All REQUIRED tags $\underline{\text { must }}$ be present in every VTF file, even when there is no value corresponding to the tag. REQUIRED tags with no values must be specified as NULL (without quotes for all variable types, including for text strings) - see discussion and table below. Of course, Processing software may not be able to function if required tags have NULL values (which are translated into a NaN (IEEE "not a number" value) or into an empty string, depending on tag type), so such values partly serve as reminders to the author that they still have values that probably should be filled in before the file is shipped. Nevertheless, if a required tag is simply unavailable, ship the file and let the users make due, "some data" generally being preferable to "no data".

Conditionally required (CONDITIONAL) tags are required in certain contexts that are explained in the comments associated with each CONDITIONAL tag. For example, when poles and zeros are used, all associated tags are then REQUIRED, including that the number of poles and zeros become required and each of the poles and zeros themselves are also required. 
Recommended tags are those which form a thoroughly documented record, but which may not be available for all data, particularly for legacy data, and are not essential to that record's use.

Optional tags are those which generally should be included if available but are not REQUIRED.

Whether a tag is REQUIRED, CONDITIONALly required, Recommended, or Optional is indicated by the comment to the immediate right of or just below the tag definition. (Please remove these annotations from working VTF files.)

Finally, because of the comments specifying this degree of requirement or need sometimes wrap past the end of the line for some of the longer-named tags and for some tag values that are long lists of possibilities provided there rather than in another table, it is often necessary to break a line that defines a tag. Please do not break these lines in actual use.

\section{Notation}

As mentioned above, angle brackets, " $<>$ ", form a description of what should be substituted at that spot. Similarly, square brackets, "[]", delineate optional or occasional items.

In addition, a construct like "[ a I b ]" means "choose either a or b, $\underline{b u t}$ you must choose at least one of them". That is, you make a choice from a list of "options", not that having a value there at all is optional.

Users familiar with Unix manuals will find this pattern familiar, while others may find it curious. The "l" character is the Boolean (logical) "OR" operator in many computer languages, thus the notion of "one OR the other". Since the notation is terse and fairly widely used, we have adopted it here. In places where it is less than obvious in meaning, we try to make that meaning clearer through examples, as in the ugly formal definition of an ISO DateTime value.

\section{Organization of this Document}

This formal definition of the VTF is presented as (1) the primary format statement given above, and (2) a large set of tables below, which define and describe the tags and supporting information. 
Table 1 enumerates the terse set of allowed variable types and their associated suffixes. (Please see the ON PRECISION section below.)

Table 2 lists all recognized physical units (we would be happy to add more as needed).

Tables 3A-3R are the bulk of the format description. These tables describe the numerous tags comprising the "header" as well as a number of lists of text strings that can be entered as the values of certain tags (for example, accelerometer-model names).

Table 4 describes the main DataSeries listing matrix (the time series or spectra). Note that some of the supporting tags in the class DataSeries are in tables 3A-3R.

\section{ISO Date-and-Time Format for DateTime Tags}

When a date \pm time variable is required, we use the "ISO 8601:2004" DateTime standard format, with a few (widely used) exceptions and permitting any degree of right-side truncation to obtain lowered precision. Please do tell the user whatever you know, even when those data do not have much precision or are incompletely known.

Our modified ISO format is

$$
\text { YYYY-MM-DD hh:mm:sS.SSS [Z|[+|-]hh [:mm] ] }
$$

As mentioned, you may use this format to any level of precision, including by using any number of fractional digits in "ss.sss" (including just "ss", the nearest second), down to the least precise DateTime, which would contain only "YYYY" (note that YYYY must always be a four-digit year in the Gregorian calendar, Common Era).

If you do not provide an explicit TimeError for a given DateTime, users are likely to infer that the actual uncertainty is implied by the degree of precision given in the DateTime value. In a particularly clear example, DateTimes given as only "YYYY-MM-DD" may reasonably be assumed to have occurred sometime during that (generally UTC) day, unless otherwise stated. (Indeed, many DateTime variables only require accuracy to one day.) In any case, this implication is 
often intentional on the part of the data provider-just be aware of the likely implications of a truncated DateTime, absent a TimeError.

The primary distinction of the VTF from the ISO standard is replacing ISO Standard's "T" between the date and time with a blank so that it reads "...-DD hh:..." rather than "...-DDThh:...", thus is a little easier on the eye. This alteration also is widely used.

(For descriptions of ISO 8601:2004, see http://www.cl.cam.ac.uk/ mgk25/iso-time.html, or the official source, http://www.iso.org/iso/en/prods-services/popstds/datesandtime.html.)

Note that the end, "[ZI[+l- $]$ hh[:mm]]" reduces to "Z" for UTC time ("Zero" or "Zulu" time). The "[+l-]hh[:mm]" part gives a time offset from UTC to any local time zone in hours and possibly minutes (a few time zones are 15 or 30 minutes off the hour). For example, "[ZI[+l-]hh[:mm]]" is "-08" (eight hours behind UTC) for Pacific Standard Time in the USA, and "+05:30" (five and a half hours ahead of UTC) for India Standard Time.

Examples: This description was finished at "2007-01-05 23:59:55Z" (rigorous ISO 8601:2004 would render that "2007-01-05T23:59:55Z"). If I only admit when this happened to the hour, to the day, to the month, or to the year, it would be "2007-01-05 23Z", "2007-01-05", "2007-01", or "2007". The latter three may also include the "Z" if desired. Absent specificity, the date are likely to be assumed to be on the local calendar (some legacy data) but more likely in UTC.

\section{On Precision: This is Important!}

The COSMOS formats (as well as Blue Book derived formats) are inherently text based and, therefore, are not referenced to any computer's internal precision, such as 64-bit IEEE floating point or 32-bit integers.

If you are creating your own VTF files, it is strongly recommended that you print floating point numbers with a sufficient number of digits to reflect the actual precision of your data, plus one or two more digits to limit round-off error (an old technique in the design of computers). 
If you are reading VTF files, be certain to allocate binary space of adequate precision in your computer so that it preserves the values presented in text of the VTF file (thus, the label "_d $\boldsymbol{d} \boldsymbol{b l}$ " suggesting use of "double precision"). Because computation is now fast and inexpensive, we recommend using double-precision or better for all $\_d b l$ and $\_c p x$ values - there is almost nothing to loose and plenty to gain.

Also because the VTF file is text, the precision is only as good as presented in the VTF file, notwithstanding “_dbl" and other notations.

\section{Null Values}

The following special values shall be used both on input and output whenever a value is unknown.

\begin{tabular}{|l|l|l|}
\hline Representation of NULL value & Comment & Most often translates into \\
\hline <some tag>_txt = NULL; & Null text-NO QUOTES & Empty string \\
\hline <some tag>_int = NULL [units]; & Null integer & $\begin{array}{l}\text { ThisFile.NullIntValue or the } \\
\text { largest allowed negative value } \\
\text { on that computer }\end{array}$ \\
\hline <some tag>_dbl = NULL [units]; & Null double precision & NaNł \\
\hline $\begin{array}{l}\text { <some tag>_cpx = NULL NULL [units]; } \\
\text { or <some tag>_cpx = NULL [units]; }\end{array}$ & Null complex double & NaN NaN \\
\hline
\end{tabular}

\$ When read into computational software, a NULL value generally will cause the parser to return a NaN (IEEE-float value "not a number") or an empty string. But in the case of integers, or on systems that do not support NaN, the values returned generally will be the largest possible (finite) negative values for that computer.

If provided in the VTF file, the three values ThisFile.NullComplexValue, ThisFile.NullRealValue, or ThisFile.NullIntValue may be returned instead, as appropriate to the computer, the application, and the wishes of the user. (All VTF parsers should include a user option for overriding these NULL value translations with other values, such as the user's own favorite "null" number, like 999. However, we strongly urge that the NULL values be a improbable value (so not 999) and that the user never use zero as a NULL value since zero is far too often a real, intended data value, not at all NULL. A maximal negative is unlikely 
in nearly all situations.) Finally, note that "maximal negative" does not mean negative infinity (which can be portrayed in IEEE floats) - this value should be reserved for its rare but valid uses.

When any VTF file is created that DOES NOT have available all of the REQUIRED tag values, then it is required that the "missing" tags be written in anyway and given the value NULL, as above. Also, these NULL values are used to fill out the "empty" header supplied at the COSMOS VDC Web site as an educational and quick-use tool. Finally, the NULL values may be used as place holders for anticipated data to be supplied later.

\section{THE COSMOS VDC TAGGED FORMAT TABLES (VTF.1.0)}

\section{Table 1. Variable-Types Suffix List}

\begin{tabular}{|c|c|}
\hline Suffix & Storage type \\
\hline$-t x t$ & $\begin{array}{l}\text { Text strings, always enclosed within double quotes (") . Use no } \\
\text { blank padding at the ends; text justification is never needed. }\end{array}$ \\
\hline -int & $\begin{array}{l}\text { Integer values }(32-\text { bit). Preferably two's complement (thus in the } \\
\text { range } \pm 2,147,483,647 \text {, that is, } \pm\left(2^{31}-1\right) \text {. }\end{array}$ \\
\hline$-\mathrm{dbl}$ & Double-precision (64-bit) floating point, preferably IEEE. \\
\hline$-c p x$ & $\begin{array}{l}\text { If a complex value is required, two dbl values are given, real } \\
\text { then imaginary, separated by white space, unless the value is } \\
\text { real, in which case either <real value> or [<real value> 0] are } \\
\text { both allowed and equivalent. }\end{array}$ \\
\hline
\end{tabular}

The significance of variable types is informational and suggestive (except as they imply particular formats for the VTF text values). The implications of these suffixes is that users should allocate comparable binary storage to accommodate the equivalent of the stated data type and precision. 
There is no longer much reason to use single-precision floating-point or complex numbers, computation being fast and inexpensive, so we urge the use of storage by using the variable types implied by these suffixes.

\section{Table 2. Recognized Units}

SI units, or at least metric units, are preferred but not required, recognizing the engineering context of strong motion data and applications.

\begin{tabular}{|c|c|}
\hline Name & Unit \\
\hline \multicolumn{2}{|r|}{ Time } \\
\hline years & \multirow{5}{*}{$\begin{array}{l}\text { Large time units used mainly to indicate TimeErrors (where not } \\
\text { implied by rounding of the associated DateTimes) }\end{array}$} \\
\hline months & \\
\hline days & \\
\hline hours & \\
\hline minutes & \\
\hline s & seconds (do not use "sec", which is not the SI abbreviation) \\
\hline $\mathrm{Hz}$ & Hertz; inverse seconds (do not use "sps") \\
\hline $\mathrm{ms}$ & milliseconds \\
\hline us & microseconds \\
\hline $\mathrm{mHz}$ & millihertz $(0.001 \mathrm{~Hz})$ \\
\hline & Gravitational acceleration \\
\hline g_local & Local Earth acceleration at the sensor GeoLocation* \\
\hline mg_local & 0.001 of local Earth acceleration \\
\hline ug_local & 0.000001 of local Earth acceleration \\
\hline g_standard & Standard Earth acceleration $(9.80665 \mathrm{~m} / \mathrm{s} / \mathrm{s})$ \\
\hline mg_standard & 0.001 of standard Earth acceleration \\
\hline ug_standard & 0.000001 of standard Earth acceleration \\
\hline
\end{tabular}




\begin{tabular}{|c|c|}
\hline \multicolumn{2}{|r|}{ Scaling } \\
\hline $\mathrm{cm} / \mathrm{g} \_l o c a l$ & centimeters offset on film record per local $\mathrm{g}$ \\
\hline $\mathrm{cm} / \mathrm{g} \_$standard & centimeters offset on film record per standard $\mathrm{g}$ \\
\hline $\mathrm{mm} / \mathrm{g} \_l o c a l$ & millimeters offset on film record per local $\mathrm{g}$ \\
\hline mm/g_standard & millimeters offset on film record per standard $\mathrm{g}$ \\
\hline in/g_local & inches offset on film record per local $\mathrm{g}$ \\
\hline in/g_standard & inches offset on film record per standard $g$ \\
\hline \multicolumn{2}{|r|}{ Linear distances and rates } \\
\hline $\mathrm{km}$ & kilometers \\
\hline $\mathrm{km} / \mathrm{s}$ & kilometers per second \\
\hline $\mathrm{mi}$ & statute miles $(1.609344 \mathrm{~km})$ \\
\hline $\mathrm{mi} / \mathrm{s}$ & statute miles per second \\
\hline $\mathrm{m}$ & meters \\
\hline $\mathrm{m} / \mathrm{s}$ & meters per second \\
\hline $\mathrm{m} / \mathrm{s} / \mathrm{s}$ & meters per second per second \\
\hline $\mathrm{cm}$ & centimeters \\
\hline $\mathrm{cm} / \mathrm{s}$ & centimeters per second \\
\hline $\mathrm{cm} / \mathrm{s} / \mathrm{s}$ & centimeters per second per second (do not use "gal") \\
\hline ft & feet $(30.48 \mathrm{~cm})$ \\
\hline $\mathrm{ft} / \mathrm{s}$ & feet per second \\
\hline $\mathrm{ft} / \mathrm{s} / \mathrm{s}$ & feet per second per second \\
\hline in & inches $(2.54 \mathrm{~cm})$ \\
\hline $\mathrm{in} / \mathrm{s}$ & inches per second \\
\hline $\mathrm{in} / \mathrm{s} / \mathrm{s}$ & inches per second per second \\
\hline um & $\begin{array}{l}\text { micrometers (do not use "microns" or " } \mu \text { "; use "u" for ASCII } \\
\text { compatibility) }\end{array}$ \\
\hline mil & 0.0001 inches, typically a machinist's unit \\
\hline \multicolumn{2}{|r|}{ Area } \\
\hline$<$ distance unit>^2 & squared <distance unit>, for example, $\mathrm{km}^{\wedge} 2$ \\
\hline
\end{tabular}




\begin{tabular}{|c|c|c|}
\hline \multicolumn{3}{|c|}{ Angles and angular rates } \\
\hline deg & degrees (always decimal) & \multirow{3}{*}{$\begin{array}{l}\text { To be used for all azimuth and } \\
\text { orientation values. }\end{array}$} \\
\hline $\mathrm{deg} / \mathrm{s}$ & degrees per second & \\
\hline $\mathrm{deg} / \mathrm{s} / \mathrm{s}$ & degrees per second per second & \\
\hline rad & radians (always decimal) & \multirow{9}{*}{$\begin{array}{l}\text { Mainly used as the units of } \\
\text { rotational time series. }\end{array}$} \\
\hline $\mathrm{rad} / \mathrm{s}$ & radians per second & \\
\hline $\mathrm{rad} / \mathrm{s} / \mathrm{s}$ & radians per second per second & \\
\hline mrad & milliradians (always decimal) & \\
\hline $\mathrm{mrad} / \mathrm{s}$ & milliradians per second & \\
\hline $\mathrm{mrad} / \mathrm{s} / \mathrm{s}$ & $\begin{array}{l}\text { milliradians per second per } \\
\text { second }\end{array}$ & \\
\hline urad & microradians (always decimal) & \\
\hline urad/s & microradians per second & \\
\hline $\mathrm{urad} / \mathrm{s} / \mathrm{s}$ & $\begin{array}{l}\text { microradians per second per } \\
\text { second }\end{array}$ & \\
\hline \multicolumn{3}{|c|}{ Miscellaneous } \\
\hline \multirow[t]{2}{*}{ counts or count } & $\begin{array}{l}\text { digital counts (the singular is used } \\
\text { compound unit name, as in Conversion }\end{array}$ & $\begin{array}{l}\text { for the denominator of some } \\
\text { Factors below }\end{array}$ \\
\hline & a simple number (unitless) & \\
\hline$\%$ & percent & \\
\hline \%FS & percent of (single sided) full scale & \\
\hline \multicolumn{3}{|c|}{ Voltage } \\
\hline $\mathrm{V}$ & \multicolumn{2}{|l|}{ Volts } \\
\hline $\mathrm{mV}$ & \multicolumn{2}{|l|}{ milliVolts } \\
\hline $\mathrm{uV}$ & \multicolumn{2}{|l|}{ microvolts } \\
\hline \multicolumn{3}{|c|}{ Conversion factors } \\
\hline$(<$ unit $>$ ) / (<unit>) & $\begin{array}{l}\text { General conversion constant from the } \\
\text { numerator's <unit>. The parentheses } \\
\text { unit is used, for example, Sensor.Se }\end{array}$ & $\begin{array}{l}\text { denominator's <unit> to the } \\
\text { are required when a compound } \\
\text { ensitivity }="(\mathbf{c m} / \mathbf{s} / \mathbf{s}) / \text { count"; }\end{array}$ \\
\hline
\end{tabular}




\begin{tabular}{|c|c|}
\hline \multicolumn{2}{|r|}{ Stress } \\
\hline $\mathrm{MPa}$ & $1,000,000$ Pascal \\
\hline $\mathrm{Pa}$ & Pascal (Newtons per square meter) \\
\hline Baryes & dynes per square $\mathrm{cm}$ \\
\hline bars & stress as bars $(0.1 \mathrm{MPa})$; roughly atmospheres \\
\hline psi & pounds per square inch (absolute) \\
\hline psig & pounds per square inch ("gauge"-relative to atmospheric) \\
\hline \multicolumn{2}{|r|}{ Energy } \\
\hline joules & SI unit of energy \\
\hline ergs & CGS unit of energy \\
\hline \multicolumn{2}{|r|}{ Energy/moment } \\
\hline $\mathrm{Nm}$ & Newton-meters \\
\hline dyne-cm & dyne-centimeters \\
\hline \multicolumn{2}{|r|}{ Strain } \\
\hline ustrain & microstrain \\
\hline \multicolumn{2}{|r|}{ Logarithmic relative power or amplitude } \\
\hline $\mathrm{dB}$ & decibels \\
\hline dB/octave & decibels per factor-of-two in frequency \\
\hline $\mathrm{dBm}$ & decibels referenced to $1 \mathrm{mV}$ \\
\hline $\mathrm{dBa}$ & decibels referenced to $1 \mathrm{~m} / \mathrm{s} / \mathrm{s}$ \\
\hline \multicolumn{2}{|r|}{ Spectra } \\
\hline$(<$ unit $>$ ) / Hz & Fourier amplitude spectrum, for example, $(\mathrm{cm} / \mathrm{s} / \mathrm{s}) / \mathrm{Hz}$ \\
\hline$(<$ unit>)/(root $\mathrm{Hz})$ & rms spectrum, for example, $(\mathrm{cm} / \mathrm{s} / \mathrm{s}) /($ root $\mathrm{Hz})$ \\
\hline$(<\text { unit }>)^{\wedge} 2 / \mathrm{Hz}$ & power spectral density, for example, $(\mathrm{cm} / \mathrm{s} / \mathrm{s})^{\wedge} 2 / \mathrm{Hz}$ \\
\hline
\end{tabular}

* The Tag Array.EarthGravity defines the value of local Earth acceleration at the Array and the Sensor, thus defining the unit "g_local". (This value is very unlikely to change across an Array, as we narrowly define it, to any degree important in strongground-motion applications. It certainly can vary across the network of one Agency, generally in the parts per thousand.) 


\section{Table 3A. Certain General and Special Header Tags}

Thisfile.Format txt $=$ "VTF.<major.minor";

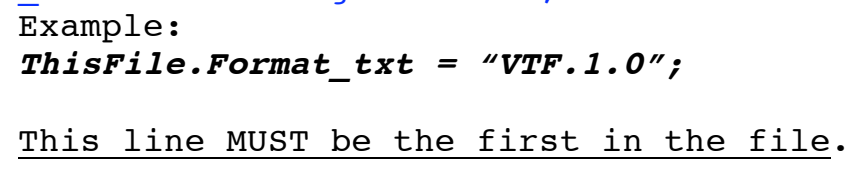

Thisfile.CharacterEncoding_txt = [ "US-ASCII" | "UTF-8" | “UTF-16" ]; Informs users and software which of the these characterencoding standards is used in this VTF file.

Ideally, this tag should be second in the file to make life easier for both parsers and humans. However, this positioning is not required (the VTF file itself is quite likely to contain a few invisible bytes at the beginning, specifying its encoding standard for use by the computer's operating system).

UTF-8 and -16 are, respectively, one- and two-byte encodings of Unicode ${ }^{\mathrm{TM}}$ (ISO-10646). UTF-8 contains all 127 of the ASCII characters, as well as most other widely used characters, this in the same storage space as simple ASCII. However, UTF-8 is incomplete in that it is missing Chinese and several other major scripts. On the other hand, UTF-16 is complete, but it uses about double the storage space of UTF-8.

Please use either UTF-8 or UTF-16 for all VTF files, giving preference to UTF-8 where practical, and to UTF-16

where comprehensiveness is needed. 
Thisfile.DataSeriesCOSMOSidentifier_txt = "<value $>$ ";

| | CONDITIONAL

REQUIRED if this file is served from the cosMOS VDC;

Optional if served directly from data owner (Agency).

The COSMOS VDC unique identifier that has been assigned

by the VDC and associated with this Dataseries. Providers

and users may both apply it subsequently for clarity, if

desired, but it must remain unchanged from the VDC's

assigned value.

Below, see Thisfile.DataSeriesHistory(n) for a means

to store a history of these unique identifiers and/or of

the filenames of precursor files.

In addition, DataSeries.AgencysIdentifier is used to

provide the provider's own identifiers for this record.

Thisfile.Preparation.Agency txt = "<value $>$ ";

|| Whatever individual, group, or Agency prepared this VTF

file; take this value from table $3 \mathrm{E}$.

Thisfile.Preparation.DateTime txt = "YYYY-MM-DD [ hh:mm:ss[z|[+|-]hh[:mm]]]";

|| The date ( \pm time) on which this cosmos tagged-format (VTF)

file was created. It is not the creation DateTime of any

source file that may be translated into or subsumed by this

VTF file nor of any data copied into this record from other

sources (for example, earthquake-source data).

Instead, use Processing.DateTime to identify the

originating-Agency's authoritative creation DateTime

for the Dataseries in this file. Various metadata also

have DateTime tags to indicate their origins separately.

Since this VTF file often will be generated from an Agency's original records kept in some other format, but not the other

way around, Processing.DateTime will not be later than

Thisfile.Preparation.DateTime. Of course, it is possible

for these two DateTimes to be identical if the originating-

Agency directly creates this tagged-format file. 
Thisfile.DataSeriesHistory [(n)]_txt = "<DataSeries ID>";

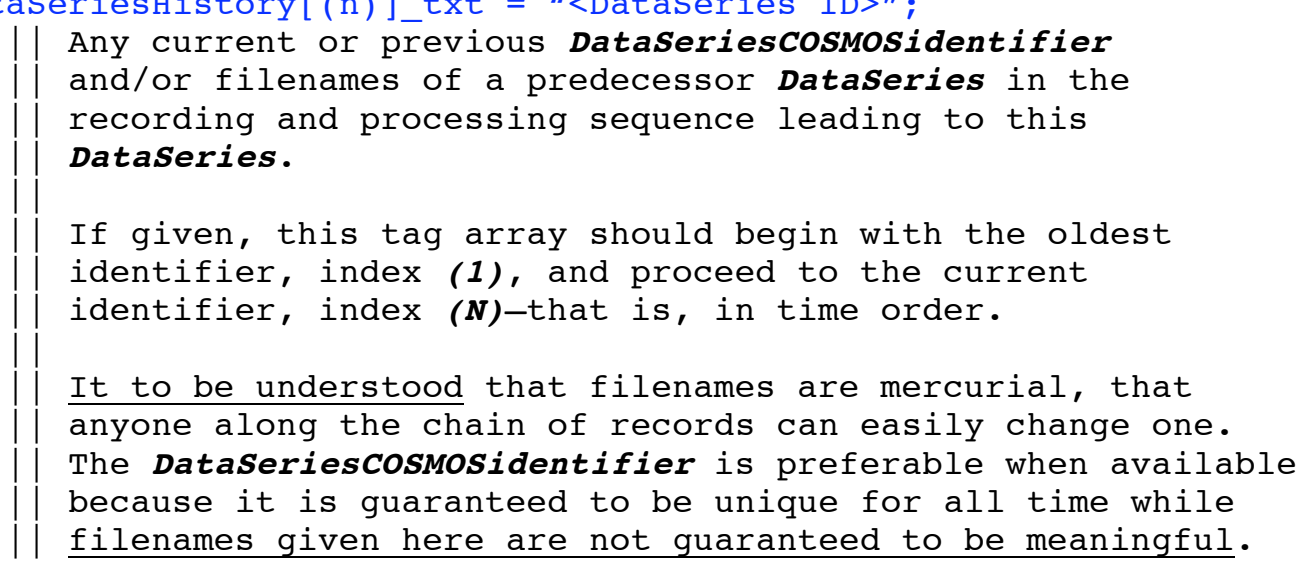

ThisFile.MetadataValid $[(\mathrm{n})]$.Start.DateTime_txt =

ThisFile.MetadataValid[ (n) ].Stop.DateTime_txt =

作

| Optional

$$
\text { "YYYY-MM-DD [ hh:mm:ss[Z|[+|-]hh[:mm] ] ]" ; }
$$

Thisfile.MetadataValid[(n)].Agency_txt $=$ "<from table $3 \mathrm{E}(\mathrm{b})\rangle "$;

As distinct from ThisFile.Preparation.DateTime, these tags provide an optional means of indicating the date ( \pm time)

interval during which the provider believes that the metadata

in this header that is supplied by the indicated Agency are

valid in their entirety (an interval often called an "epoch").

Users occasionally request such epochal information.

If either or both the Start.DateTime and the Stop.DateTime

is/are given, then the associated Agency is REQUIRED

(meaning, of course, that the stated epoch applies only

to metadata generated by said Agency). 
The most common application of these epochal tags is likely to be just leaving them out, thus accepting their default values.

The default value of either tag is effectively the moment of VTF-file creation but specifically nearer end of this file's Dataseries, with no other promises made. That is, the values are valid for the duration of the Dataseries.

If given at all, it is likely that one set of these epochal tags will be given, and is likely to (but not required to) refer to the same Agency as Thisfile.Preparation.Agency, the author of this VTF file. That is, the author of this VTF file is not likely to make any promises for other suppliers of metadata, such as earthquake Hypocenter data.

Thisfile.ValidBand.HighPass.Corner_dbl = <value $\rangle[\mathrm{Hz} \mid \mathrm{s}]$;

Thisfile.ValidBand.LowPass.Corner $\overline{d b l}=\langle$ value $\rangle[\mathrm{Hz} \mid \mathrm{s}]$;

Thisfile.ValidBand.Agency txt $=$ "<from table $3 \mathrm{E}(\mathrm{b})>$ ";

Thisfile.ValidBand.Comments_txt $=$ "<Comments $>$ ";

In the event that these are preliminary ("quick release")

Dataseries, it is often the case that response spectra and other information are valid only over a limited bandwidth. If this is so, we recommend an explicit statement of the valid frequency or period band via these tags. Obviously, HighPass and LowPass have opposite meanings when expressed in period than when they are expressed in frequency.

See also, Processing.HumanReview, Processing.Stage,

Processing.Constantsused, and Processing.Problem.Status.

While we think them unlikely to be needed, for completeness we provide space for both Comments and an Agency associated with this band-limit determination. (If not specified here, the Agency may safely be assumed to be the Processing.Agency.) 
Thisfile.NullComplexValue_dbl = <value $\rangle\langle$ value $>$;

ThisFile.NullRealvalue_db $\bar{l}=\langle$ value $>$;

Thisfile.NullIntValue int $=\langle$ value $\rangle$;

| These values are not used in the VTF per se. They are used only as "option two of four" (below) for parser handling of

NULL tag values.

For NULL-valued tags, from high to low priority:

For NULt-valued tags, from high to low priority:

"Option one" is to use user-supplied values as given to the VTF reading routine; these are to override the other three "options" though such action is not recommended because of the potential for value conflicts.

"Option two" is to use the values given by these ThisFile.Null... tags for numerics and empty strings for _txt. (That is, if you desire options three or four, do not provide these tags or remove them from a file sent to you.)

"Option three", the preferred one, is to use an IEEE NaN for _dbl and a pair of these for _cpx values, empty strings for _txt values, and the largest possible negative integer (in most cases, [1 - 2^31] = $-2,147,483,647$ ) for _int

"Option four" is to use the largest possible negative values on that computer for all numerics and empty strings for _txt.

Note that with "Option one of four" all parsers must allow users to override these three NULL-values, as well as the preferred default of "Option three". 
Thisfile.Annotations [ (m) ].TextValue_txt $=$ "<Comments $>$ ";

Thisfile.Annotations [ (m)].Agency_txt $=$ "<from table $3 \mathrm{E}(\mathrm{b})\rangle "$;

Agency which contributed the comments Annotations(m).Textvalue

and those comments. Agency is REQUIRED if TextValue is present.

(That is, it is wise to identify the source of each Textvalue

because those comments can come from multiple Agencies.)

If you need more than one line of text put it all into one,

long string with "lines" separated by the string "||n|".

Thisfile.Citation_txt $[(\mathrm{n})]=$ "<citation $>$ ";

Thisfile.URL txt $[(\mathrm{n})]=$ "<Web address $>"$.

| If there is/are report(s) available for this Thisfile,

provide a citation with one or both of these tags.

Therefore, typical VTF files will begin with:

Thisfile.Format txt $=$ "VTF.1.0";

Thisfile.DataSeriesCosmosidentifier_txt = "<value>";

ThisFile.Preparation.Agency_txt = "<value $\rangle$ ";

Thisfile.Preparation.DateTime txt = "YYYY-MM-DD";

where the various values are inserted to identify this record and its origins.

\section{Table 3B(a). Type of DataSeries}

DataSeries.PhysicalParameter txt $=$ "<from table 3B(b) $>$ ";

| | REQUIRED

Units are givē by DataSeries.OrdinateUnits in table $3 R$, not here.

This tag provides a means of giving a somewhat more detailed text

description of the type of data than units alone would provide.

DataSeries.Physicalparameter is in the style of the Blue Book

text description of the data (table 3B(b)). 
DataSeries.NonLinearComputation [ (m) ]. Description_txt = "<value $>$ "; DataSeries.NonLinearComputation [ (m) ].URL_txt $=$ "<Web address $>$ ";
At least one such tag is REQUIRED if the physical parameter
is a nonlinear response spectrum or the result of some other nonlinear computation, in which case the method of computation is to be described here, either by using a well-known name or a citation (Description) or a Web site (URL) containing a similar description of the computation.

\section{Table 3B(b). Codes for Types of DataSeries}

\begin{tabular}{|c|c|c|c|}
\hline Allowed values & * & Meaning & $\begin{array}{c}\text { Typical units for } \\
\text { DataSeries.Ordinateunits }\end{array}$ \\
\hline "UnProcessed Acceleration" & A & $\begin{array}{l}\text { "Volume 1" linear acceleration } \\
\text { with calibrations applied but } \\
\text { baseline NOT processed, though } \\
\text { a simple mean may be removed }\end{array}$ & $\begin{array}{l}\mathrm{cm} / \mathrm{s} / \mathrm{s}, \text { g_local, counts, } \\
\text { and so forth }\end{array}$ \\
\hline "Processed Acceleration" & A & $\begin{array}{l}\text { "Volume 2" linear acceleration } \\
\text { with baseline fully processed, } \\
\text { ready for double integration, } \\
\text { possibly filtered too. }\end{array}$ & $\begin{array}{l}\mathrm{cm} / \mathrm{s} / \mathrm{s}, \text { g_standard, } \\
\text { counts, and so forth }\end{array}$ \\
\hline "Calibration Input Signal" & CAL & $\begin{array}{l}\text { A signal input to the named } \\
\text { instrument to calibrate it (in } \\
\text { any appropriate units). }\end{array}$ & $\begin{array}{l}\mathrm{cm} / \mathrm{s} / \mathrm{s}, \mathrm{cm} / \mathrm{s}, \mathrm{cm}, \\
\text { g_local, counts, and so } \\
\text { forth }\end{array}$ \\
\hline $\begin{array}{l}\text { "Acceleration Correction } \\
\text { Baseline" }\end{array}$ & $\mathrm{BL}$ & $\begin{array}{l}\text { t A full time series of the } \\
\text { total corrections applied to a } \\
\text { "Volume 1" acceleration time } \\
\text { series to generate an } \\
\text { acceleration time series which } \\
\text { integrates successfully into } \\
\text { the same velocity and } \\
\text { displacement time series } \\
\text { reported by the providing } \\
\text { Agency. }\end{array}$ & $\begin{array}{l}\mathrm{cm} / \mathrm{s} / \mathrm{s}, \text { g_standard, } \\
\text { counts, and so forth }\end{array}$ \\
\hline
\end{tabular}




\begin{tabular}{|c|c|c|c|}
\hline "Velocity" & $\mathrm{V}$ & Linear velocity & $\mathrm{km} / \mathrm{s}, \mathrm{m} / \mathrm{s}, \mathrm{cm} / \mathrm{s}, \mathrm{in} / \mathrm{s}$ \\
\hline "Absolute Displacement" & $\mathrm{D}$ & Linear absolute displacement & $\mathrm{m}$, $\mathrm{cm}$, in \\
\hline "Relative Displacement" & $\mathrm{D}$ & $\begin{array}{l}\text { Linear relative displacement } \\
\text { (for example, displacement } \\
\text { computed from an acceleration } \\
\text { record) }\end{array}$ & $\mathrm{m}$, $\mathrm{cm}$, in \\
\hline "Rotational Acceleration" & RA & Angular acceleration & $\mathrm{deg} / \mathrm{s} / \mathrm{s}$ \\
\hline "Rotational Velocity" & RV & Angular velocity & $\mathrm{deg} / \mathrm{s}$ \\
\hline "Rotational Displacement" & $\mathrm{RD}$ & Angular displacement & deg \\
\hline "Absolute Pressure" & $\mathrm{P}$ & Absolute pressure & $\mathrm{Pa}$, Baryes, psi \\
\hline "Relative Pressure" & GP & $\begin{array}{l}\text { "Gage pressure", that is, } \\
\text { pressure relative to } \\
\text { atmospheric }\end{array}$ & Pa, Baryes, psi \\
\hline "Volumetric Strain" & $\mathrm{S}$ & Volumetric strain & ustrain \\
\hline "Linear Strain" & $\mathrm{S}$ & Linear strain & ustrain \\
\hline "Response Spectra (Sa)" & $\mathrm{Sa}$ & & $\mathrm{cm} / \mathrm{s} / \mathrm{s}$, and so forth \\
\hline “Response Spectra (Sv)” & Sv & Sa, Sv, Sd, pseudovelocity & $\mathrm{cm} / \mathrm{s}$, and so forth \\
\hline “Response Spectra (Sd)" & Sd & $(\omega * \mathrm{Sd}=\mathrm{PSV})$, or & $\mathrm{cm}$, and so forth \\
\hline “Response Spectra (PSA)” & PSA & pseudoacceleration $\left(\omega^{*} \omega *\right.$ Sd $=$ & $\mathrm{cm} / \mathrm{s} / \mathrm{s}$, and so forth \\
\hline “Response Spectra (PSV)" & PSV & & $\mathrm{cm} / \mathrm{s}$, and so forth \\
\hline "Geometric Mean PSA" & gPSA & $\begin{array}{l}\text { Geometric mean of horizontal- } \\
\text { component linear PSAs. }\end{array}$ & $\mathrm{cm} / \mathrm{s} / \mathrm{s}$, and so forth \\
\hline "Median PSA over 90 degrees" & mPSA & $\begin{array}{l}\text { Median linear PSA for } \\
\text { horizontal component rotated } \\
\text { through } 90 \text { degrees, preferably } \\
\text { in one-degree increments. }\end{array}$ & $\mathrm{cm} / \mathrm{s} / \mathrm{s}$, and so forth \\
\hline
\end{tabular}




\begin{tabular}{|c|c|c|c|}
\hline $\begin{array}{l}\text { "Nonlinear Response Spectra } \\
\text { (Sa)" }\end{array}$ & $\mathrm{nSa}$ & \multirow{5}{*}{$\begin{array}{l}\text { Nonlinear response spectra. } \\
\text { Describe method of computation } \\
\text { in DataSeries.NonLinearCompu- } \\
\text { tation.Description or .URL. }\end{array}$} & $\mathrm{cm} / \mathrm{s} / \mathrm{s}$, and so forth \\
\hline $\begin{array}{l}\text { "Nonlinear Response Spectra } \\
(\mathrm{Sv}) \text { " }\end{array}$ & $\mathrm{nSv}$ & & $\mathrm{cm} / \mathrm{s}$, and so forth \\
\hline $\begin{array}{l}\text { "Nonlinear Response Spectra } \\
\text { (Sd)" }\end{array}$ & $\mathrm{nSd}$ & & $\mathrm{cm}$, and so forth \\
\hline $\begin{array}{l}\text { "Nonlinear Response Spectra } \\
\text { (PSA)" }\end{array}$ & nPSA & & $\mathrm{cm} / \mathrm{s} / \mathrm{s}$, and so forth \\
\hline $\begin{array}{l}\text { "Nonlinear Response Spectra } \\
\text { (PSV)" }\end{array}$ & nPSV & & $\mathrm{cm} / \mathrm{s}$, and so forth \\
\hline $\begin{array}{l}\text { "Geometric Mean Nonlinear } \\
\text { PSA" }\end{array}$ & gnPSA & $\begin{array}{l}\text { Geometric mean of horizontal- } \\
\text { component nonlinear PSAs. }\end{array}$ & $\mathrm{cm} / \mathrm{s} / \mathrm{s}$, and so forth \\
\hline $\begin{array}{l}\text { "Median Nonlinear PSA over } 90 \\
\text { degrees" }\end{array}$ & mnPSA & $\begin{array}{l}\text { Median nonlinear PSA for } \\
\text { horizontal component rotated } \\
\text { through } 90 \text { degrees, preferably } \\
\text { in one-degree increments. }\end{array}$ & $\mathrm{cm} / \mathrm{s} / \mathrm{s}$, and so forth \\
\hline $\begin{array}{l}\text { "Acceleration Amplitude } \\
\text { Spectrum" }\end{array}$ & DFT & Absolute value of DFT & \multirow{2}{*}{ for example, $(\mathrm{cm} / \mathrm{s} / \mathrm{s}) / \mathrm{Hz}$} \\
\hline $\begin{array}{l}\text { "Acceleration Complex } \\
\text { Spectrum" }\end{array}$ & CFT & Complex DFT Spectrum & \\
\hline "Acceleration RMS Spectrum" & RMS & $\begin{array}{l}\text { RMS of noise in acceleration } \\
\text { (square root of power } \\
\text { spectrum) }\end{array}$ & $\begin{array}{l}\text { for example, } \\
(\mathrm{cm} / \mathrm{s} / \mathrm{s}) /(\text { root } \mathrm{Hz})\end{array}$ \\
\hline “Acceleration Power Spectrum" & PWR & $\begin{array}{l}\text { Power spectrum of Events or } \\
\text { noise recorded in acceleration }\end{array}$ & $\begin{array}{l}\text { for example, } \\
(\mathrm{cm} / \mathrm{s} / \mathrm{s})^{\wedge} 2 / \mathrm{Hz}\end{array}$ \\
\hline \multicolumn{4}{|c|}{ <similarly for "Velocity", and so forth> } \\
\hline “ & “_” & $\begin{array}{l}\text { USER-EXTENSIBLE LIST ( use } \\
\text { established, terse, clear, } \\
\text { English names or phrases which } \\
\text { must begin with "User's } \\
\text { description: ") }\end{array}$ & $\begin{array}{l}\text { <time-series or spectral } \\
\text { units }>\end{array}$ \\
\hline
\end{tabular}


*Abbreviations recommended for use in VTF filenames to identify type of trace contained in the file.

†Corrections to preliminary velocity and displacement time series can be and should be translated back to the original acceleration record, and this corrected acceleration record ideally should be integrated to produce the processed velocity and displacement DataSeries actually distributed. Some authors prefer to correct, integrate, correct, and integrate again, however this process does not yield an acceleration trace that integrates correctly to displacement, and the practice is discouraged. It is easiest for users if corresponding acceleration, velocity, and displacement time series are related to each other by simple, trapezoidalrule integration.

\section{Table 3C(a). Record Types}

DataSeries.Cause txt $=$ "<value $>$ "

A value from the upper three segments of table $3 \mathrm{C}(\mathrm{b})$

describing what caused this record to be recorded. Note

that some coordination is required with physicalparameter.

DataSeries.Calibration.InputType txt $=$ "<value $>$ ";

|| If DataSeries.Cause_txt is a "... Calibration", then this

tag is also REQUIRED and must be equal one of the values in

the last segment of table $3 \mathrm{C}(\mathrm{b})$ or to a user-created name.

DataSeries.Calibration. InputAmplitude[(n)]_dbl = <value $\rangle\langle$ units $>$;

Recommended for "Step", "Square Wave", and "Random Steps"

calibrations whenever the value is known. The (n) here

and in the next tag may be used when the values vary

during the calibration, as in "Random Steps".

DataSeries.Calibration. InputstepDuration [(n)]_dbl = <value $>$ s;

Recommended for "Square Wave" Calibrations if known and

for "Random Steps" if at all possible. To accommodate

the latter, this is the half period, the duration of a peak

or trough.

DataSeries.Calibration.InputURL txt $=$ "<Web address $>$ "

|| Recommended for signals that are too complex to describe

well here-Web address(es) of a formal description, which

may be a time series. Indeed, this URL may point to a

"Calibration Input Signal" (CAL) type of VTF file.
|| REQUIRED

| | CONDITIONAL

| | CONDITIONAL

|| Optional

|| Optional 


\section{Table 3C(b). Record-Type and Input-Type Codes}

\begin{tabular}{|c|c|}
\hline Record type & Meaning \\
\hline \multicolumn{2}{|c|}{ Use any of the following as values for Dataseries.Cause: } \\
\hline "Seismic Trigger" & An Earth signal triggered the recorder \\
\hline "Remote Trigger" & A radio, landline, or other external trigger \\
\hline "Preset Trigger" & A timed, preset trigger \\
\hline "Manual Trigger" & A human finger on a button at the $\boldsymbol{D A U}$ \\
\hline "Function Test" & Some recorder-functionality test other than calibration \\
\hline \multicolumn{2}{|l|}{ (Avoid the following four) } \\
\hline "Sensor Calibration" & The senser is being calibrated \\
\hline "Amplifier Calibration" & The amplifier is being calibrated \\
\hline "Recorder Calibration" & The recorder as a whole is being calibrated \\
\hline "Other Calibration" & Some other element of the system is being calibrated? \\
\hline \multicolumn{2}{|c|}{ (Preferred calibration "Event" names) } \\
\hline $\begin{array}{l}\text { "Sensor Through Recorder } \\
\text { Calibration" }\end{array}$ & $\begin{array}{l}\text { A Calibration pulse or sequence is introduced into the sensor } \\
\text { and recorded after the ADC (or equivalent location for analog } \\
\text { recorders). }\end{array}$ \\
\hline $\begin{array}{l}\text { "Preamplifier Through Recorder } \\
\text { Calibration" }\end{array}$ & $\begin{array}{l}\text { A Calibration pulse or sequence is introduced into the first- } \\
\text { stage amplifier and recorded after the ADC or equivalent. }\end{array}$ \\
\hline $\begin{array}{l}\text { "Filter Through Recorder } \\
\text { Calibration" }\end{array}$ & $\begin{array}{l}\text { A Calibration pulse or sequence is introduced after the } \\
\text { amplifiers but before the (anti-alias) filters and recorded } \\
\text { after the ADC or equivalent. }\end{array}$ \\
\hline "Recorder Calibration" & $\begin{array}{l}\text { A Calibration pulse or sequence is introduced into the ADC } \\
\text { inputs (after the final amplifiers and filters for analog). }\end{array}$ \\
\hline
\end{tabular}




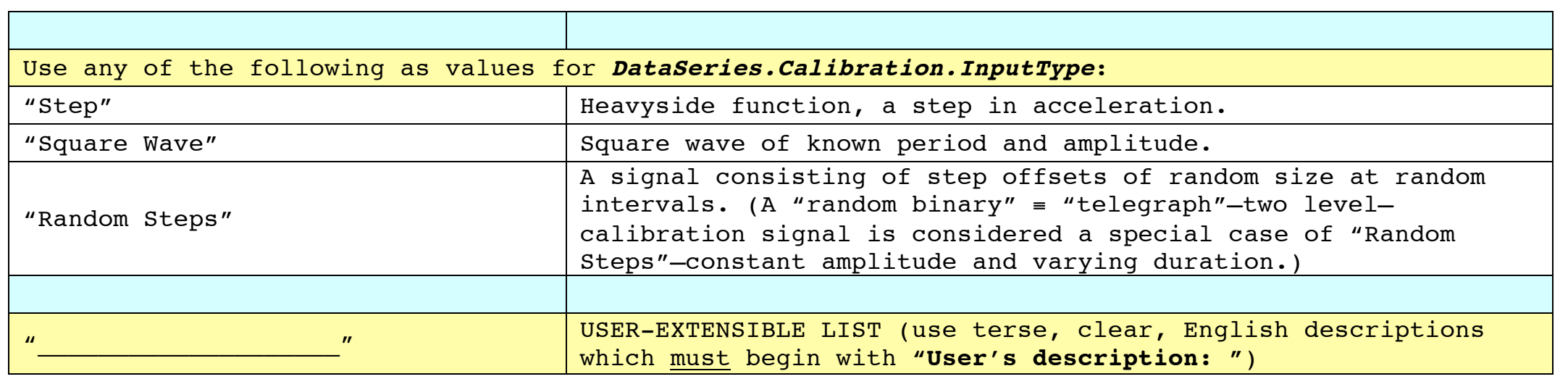

NOTE: The lined-out items and their proposed replacements need to be reconsidered with proper consultation by electrical engineers. What is the intended distinction between an "amplifier" and "recorder" calibration here? How does one calibrate the amplifier, but not the ADC (analog-to-digital converter)? It can be done by elimination, or by isolation from the ADC and recorder, but most systems cannot do this - it is a laboratory test. More discussion is needed and the distinctions need to be drawn here. We offer the subsequent suggestions, which follow the points at which most systems (DASs) allow signals to be injected and recorded.

\section{Table 3D. Geographic Naming Information}

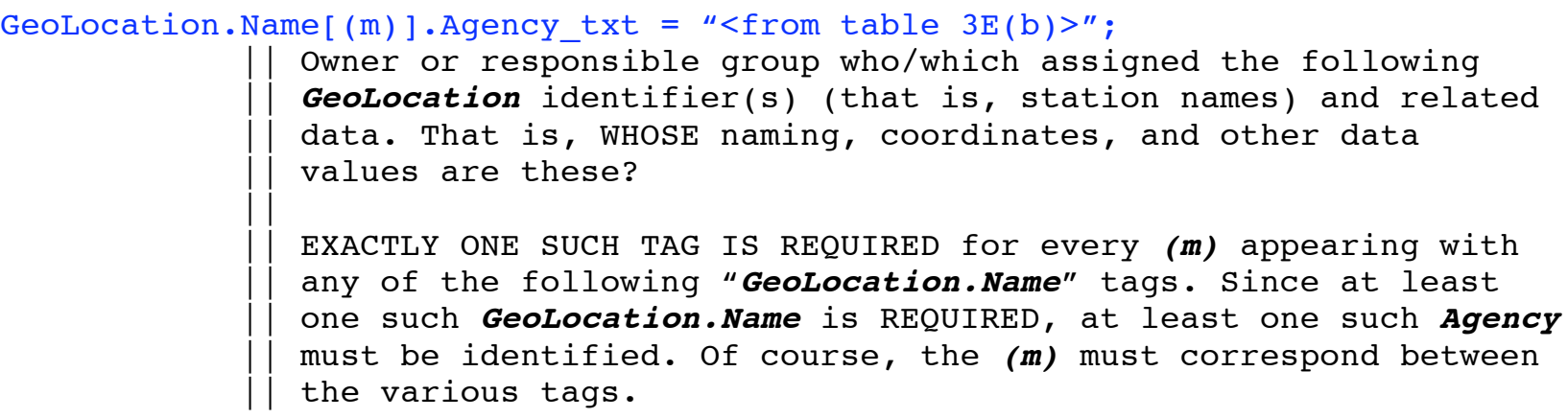


At least one of GeoLocation.Name.SNCL, GeoLocation.Name.ExpandedSNCL,

or GeoLocation.Name.ShortName is REQUIRED. More than one may be given.

GeoLocation.Name [ (m) ].SNCL_txt = "SSSSS.NN.CCC.LL";

|| The FDSN SNCL format "Station.Network.Channel.Location";

Geolocation name. (In SNCL terminology, Geolocation

would be "Station", hence "SNCL" and "SSSSS".)

GeoLocation.Name [ (m) ].ExpandedSNCL txt $=$ "SSSSSS.NN.CCC.LLL"

For use when the usêer would like to follow the SNCL format

but doing so is not practical because field lengths are

longer than those defined formally by FDSN. For example,

a six-digit or longer Geolocation.Name or long "Location"

(LL) fields designating channels within large Arrays (and

certainly across the network operated by one Agency) will require larger SNCL fields.

The field lengths for "SSSSSS" and "LLL" are shown only for illustration. There is no limit on the length of any field except "CCC", which should follow FDSN conventions. We also recommended that the "NN" field be used as defined by FDSN (therefore, if your network does not yet have an FDSN code, we recommend that you obtain one-it's easy and FDSN codes are widely recognized as coordinated, international network symbols).

The ExpandedSNCL is the VTF preferred solution for names not strictly fitting within the FDSN SNCL format. ExpandedSNCL is self-contained, systematic, and a format familiar to many users, as well as having some potential for compatibility with future FDSN naming evolution.

|| CONDITIONAL

|| CONDITIONAL 
Whether this extension will be adopted by the FDSN is uncertain, but we recommend it in order to be more accommodating of

international practices, such as increasingly common use of

Geolocation names longer than five characters, as well as

increasingly ambitious networks with large numbers of sensors,

potentially into the thousands.

GeoLocation.Name $[(\mathrm{m})]$. ShortName txt $=$ "<value $>$ ";

| Any other alpha, numeric, or mixed name of any length, but generally short. (This tag will often be used as a primary name, but note that SNCL or ExpandedSNCL are the

preferred naming formats for Geolocations in the VTF.)

GeoLocation.Name [ (m) ]. Index_txt = "<value $>$ ";

| This tag may be REQUIRED in the context of some Arrays,

| | CONDITIONAL

as when neither SNCL nor ExpandedSNCL is used and a single

ShortName identifies an entire Array. (If a SNCL name

lacking a sufficient "LL" field is used, Index also may

be needed.) In such cases, Index ( \pm "CCC" codes) must be

used to identify particular locations and component

orientations within the Array.

Index generally is used in conjunction with shortName and only rarely with SNCL or ExpandedSNCL names. This tag

is included here principally for backward compatibility.

We urge that SNCL or ExpandedSNCL names be used wherever

feasible.

GeoLocation.Name [ (m) ].Description_txt = "<value $>$ ";

A longer description of Geolocation coordinates, such as

a geographic place name with a terse site description

(for example, "Left abutment of ... dam").

Many institutions subdivide these Descriptions into several parts, each assigned to different variables,

cells, or lines for their internal processing uses.

For the VTF, please indicate these divisions with

the VTF new-line sequence, "| $|\mathbf{n}| "$. 


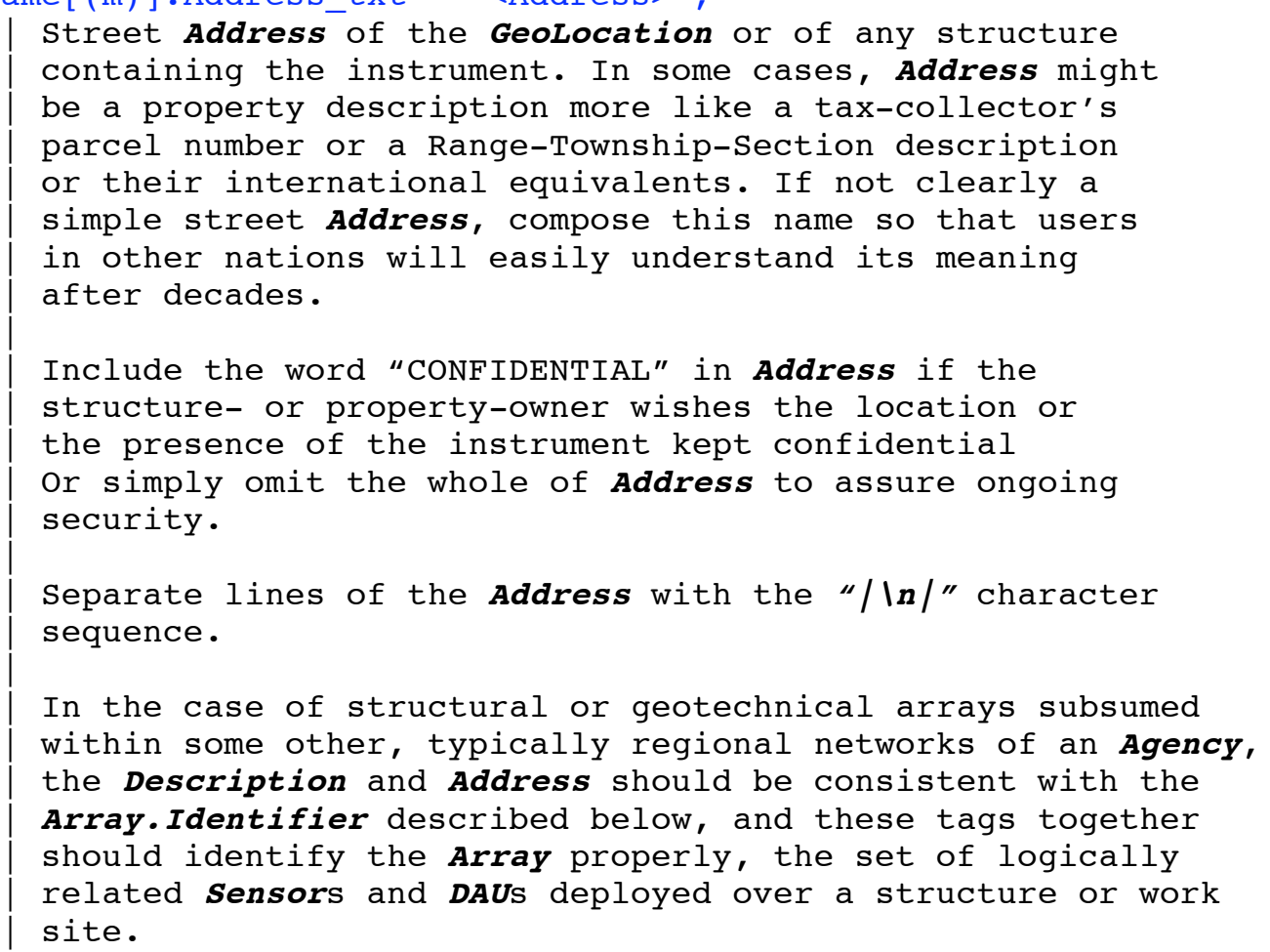

\section{On Sensor GeoLocation Naming}

There are three ways of naming a GEOGRAPHIC THREE-DIMENSIONAL LOCATION, and at least ONE of these CONDITIONAL tags is REQUIRED: GeoLocation.Name.SNCL, ExpandedSNCL, or ShortName. The SNCL or ExpandedSNCL tags are the preferred VTF naming formats. 
(Users of numeric names please note that "SNCL" names are entirely compatible with numerical names. Simply represent the numerals as characters and put them into the "SSSSSS" string. In any case, a string representation is inherent throughout VTF and does not abandon either generality or specificity.)

The SNCL name was carefully thought out by FDSN and IRIS and is quite general. Thus, a large proportion of names can be translated into a compatible SNCL name without loss of generality. (Sadly, however, the "SSSSS" portion is too short for a number of naming formats which use six or more characters in their primary GeoLocation.Name code. There are circumstances in strong-motion work (for example, structural and geotechnical Arrays) where one could make good use of a significantly longer "LL" field to name components within an Array. It is for these purposes that we provide ExpandedSNCL.) Nevertheless, numeric names certainly are included in the SNCL format up to five digits (and in ExpandedSNCL up to any length). Unique two-character network names (the "N" of "SNCL") are available through FDSN for use in these names. We use these FDSN codes extensively elsewhere in the VTF to identify networks and we urge their adoption by all strong-motion networks.

The other method of naming a LOCATION is GeoLocation.Name.ShortName, which provides for text, numeric, or mixed strings of any type and size, often with Index and additional details.

With any of these three primary naming tags, the user may include the Index tag. However, this "sub-name" tag, equivalent to the "LL" field of SNCL or ExpandedSNCL, is given primarily for backward compatibility. We urge that Index not be used and that SNCL names be used in their stead. (Index replaces the "Location Index of sensor site" is COSMOS v1.20 fixed-field format Sensor information and used to identify Sensor elements within Arrays.)

The name tag GeoLocation.Name.Description is for an additional narrative description, and may be used, if desired. It is not a substitute for using one of the three primary naming tags (SNCL, ExpandedSNCL, or ShortName). This "Long Description" is the former "Station identification" of COSMOS v1.20 and is meant to contain a descriptive naming phrase, sometimes the same name that is abbreviated in an alphabetic GeoLocation name (for example, the SNCL "SSSSS" field). An example of these descriptive names is "CA: Murietta Hot Springs; Skinner Dam, Left Abutment, Control Bldg." for the USGS NESMP station with "SSSSS" code "0720".

Note that because one can use Subscripts, there is no need for the various aliases and alternate names that existed in COSMOS v1.20. Simply create more GeoLocation.Name(n).ShortName tags required to provide all names of that point 
in 3D space. Each of these tags requires a corresponding Name(n).Agency to identify that Name's owner and/or creator. Between them, ShortName, SNCL and ExpandedSNCL replace "Station number", "Station alphanumeric code", and "Secondary station number" of the COSMOS fixed format v1.20. Similarly, Subscripts and the two tags GeoLocation.Name(m).Agency and DateTime offer the necessary mechanism for describing the timing of name changes, any secondary naming, resurveying of a GeoLocation, small moves of a GeoLocation, and any out-of-date names that may have been used in the past for a given GeoLocation.

\section{SNCL Naming Details}

GeoLocation.Name.SNCL names must follow the FDSN SNCL format rules while ExpandedSNCL must mimic those rules closely, including use of the same "CCC" sensor orientation-and-type ("channel") codes. For ExpandedSNCL, the "SSSSS" and "LL" (and, reluctantly, the "NN") fields may be lengthened as needed, but should remain as terse as is compatible with the data-provider's practices. If either of these SNCL tags is used as the primary GeoLocation.Name, then the network codes in Array.Agency and GeoLocation.Name.Agency should agree with the network-code field ("NN") of the SNCL name except, perhaps, in rare cases of multiple names for the same GeoLocation used by different Agencies.

The FDSN SNCL naming conventions are: Any five or fewer alpha and/or numeric characters for the GeoLocation name "SSSSS"; an FDSN-assigned two-character "network" code, "NN"; a SEED-format "channel" code, "CCC"; and, optionally, a two-character "tie-breaking" "location" code, "LL", to be used in cases where you have more than one channel with the same "SSSSS.NN.CCC" name (as will often be the case in structural and geotechnical Arrays). An "LL" might be used, for example, if one specified a structure by "SSSSS.NN", a location within a structure by "LL", and the orientation and type of sensor at that location by "CCC". (Since many components inside a structure point the same direction, there will routinely be conflicting names unless one includes the "LL" code to distinguish specific locations [and/or orientations] within the structural Array.)

For the SEED-format channel code, "CCC", consult Appendix A of the SEED manual, which may be downloaded from http://www.iris.edu/manuals/SEEDManual V2.4.pdf. Succinctly, the three letters correspond to (1) a frequency band, (2) the type of Sensor, and (3) the orientation of the Sensor. The most common SEED-format CHANNEL codes for accelerometers sampled at $80 \mathrm{~Hz}$ or higher would be "HNZ", "HNN", and "HNE", for truly cardinally oriented 
components, or "HNZ", "HN2", and "HN3" for a vertical and two non-cardinal, orthogonal, horizontals, and possibly "HNZ", "HNT", and "HNR" for a beam-forming Array or components rotated toward the source azimuth ("R" and "T" meaning "Radial" and "Transverse" in this context), as will be the case for some geotechnical Arrays. The "N" means "acceleration", and the "H" means a lower-corner period of $10 \mathrm{~s}$ or longer and a sampling rate of $80 \mathrm{~Hz}$ or faster.

Note that SNCL names are case sensitive, but there are no rules in the "SSSSS" part about what order or amount of alphas and numerics there may be. In other words, users may put five-digit or shorter numeric names into $S N C L$ and any six-digit or longer numeric names into the ExpandedSNCL name.

If you are using not ExpandedSNCL or SNCL (that is, you are using only ShortName, possibly in conjunction with Index), then you must also include an Array.Agency identification tag from table 3E(b) (the value generally should equal the value of GeoLocation.Name.Agency). In all cases, the FDSN/IRIS/SNCL network name code (the second column in table $3 \mathrm{E}(\mathrm{b})$ ) is strongly preferred in this application also, since it is assigned and recognized through international agreements.

\section{Table 3E(a). Array Variables, including Network/Owner/Agency Codes}

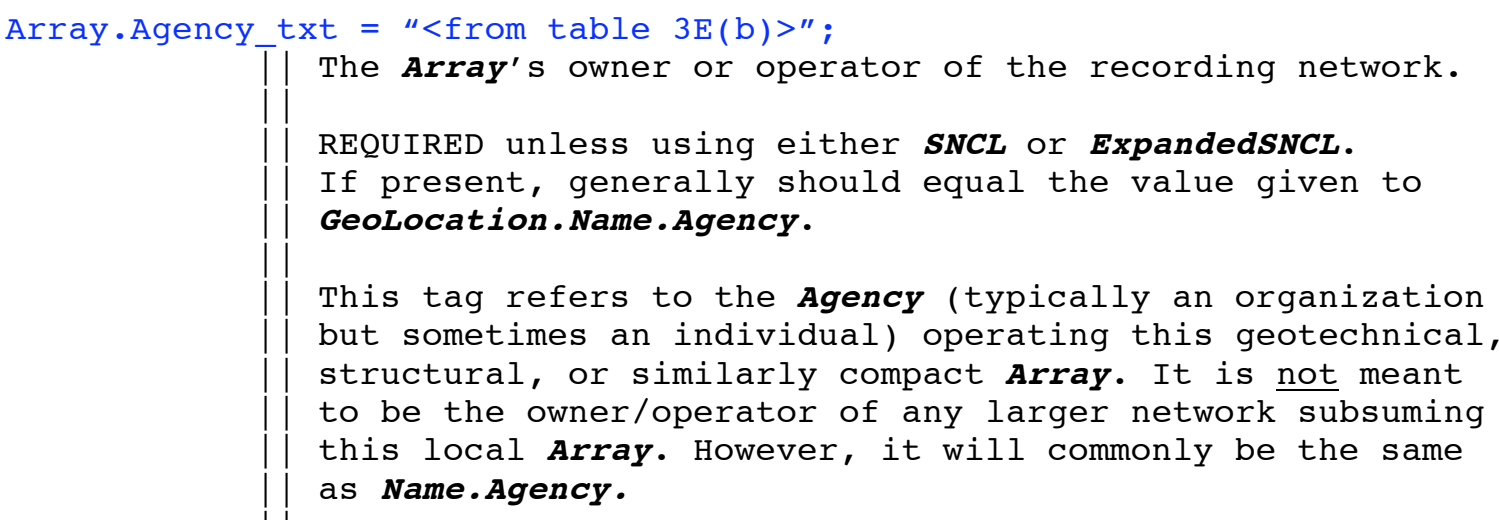


(For example, although the USGS's NESMP operates many structural Arrays, of various names or addresses, the Array.Agency, Name.Agency, and Processing.Agency tags will all be "NP". In contrast, both the NGA and PEER data sets would normally use a different value for Processing.Agency-themselves, because they do their own processing of other Agencies' records.)

CAUTION: The DateTime associated with this Agency is assumed to be the same as Processing.DateTime.

Array.Identifier_txt $=$ "<value $>$ ";

If the structural, geotechnical, or otherwise distinct

|| Optional

Array of which this instrument is part has some overarching

name other than that associated with the GeoLocation.Name

given in Array.Agency, provide it here.

Make this Identifier consistent with the values of

Geolocation.Name.Description and Address when these tags

also describe structural and other local Arrays.

Array. Owner txt $="<$ from table $3 \mathrm{E}(\mathrm{b})>"$;

| Table 3E identification of Array owner, if different

from Array.Agency. It is unlikely that this tag will

be any more than rarely used since nearly all Arrays

have a name or code that implies the owner.

Array. Operator txt $="<$ from table $3 \mathrm{E}(\mathrm{b})>"$.

| Table $3 \mathrm{E}$ identification of Array operator, if different

from Array.Agency or Array. Owner.

Array.Affiliate txt = "<from table $3 \mathrm{E}(\mathrm{b})>"$;

|| Identification of some other Array affiliate, if the above

names are an incomplete list of Agencies associated with

this Array.

The three tags just above are partially conflicting with Array.Agency and ThisFile.MetadataValid.Agency and are only given to provide backward compatibility; their use is not recommended. 

units "g_local", "mg_local", or "ug_local" are used anywhere including if the values of the time series used here were converted from one originally expressed in g_local. In other words, Array.EarthGravity may be the scaling factor used to convert "Volume 1" units of g local into absolute units such as $\mathrm{cm} / \mathrm{s} / \mathrm{s}$ when the original is only known in units of local Earth acceleration, and, if so, this conversion factor must be given.

RawSeries.GravityCalibratedAgainst_txt = [ "g_local" | "g_standard" ]; RawSeries.GtoGalConversionConstant txt = [ "g local"| "g standard" ]

|| Both are REQUIRED whenever the RawSeries was converted from acceleration values expressed in "g local" or "g standard" into absolute units such as "cm/s/s".

In some (many?) cases, unfortunately, these two values will differ, as when the instrument is calibrated against a local Earth-acceleration value (generally by a flip test at the site), but they are converted with the standard value, $980.665 \mathrm{~cm} / \mathrm{s} / \mathrm{s}$. Although this distinction generally makes only a several part-per-thousand difference and is smaller than some other uncertainties, it is best to document it.

RawSeries.GravityCalibratedAgainst indicates whether values of acceleration given in this VTF file as absolute units like "cm/s/s" originally were expressed (generally in the RawSeries) in units of either "g standard" $(980.665 \mathrm{~cm} / \mathrm{s} / \mathrm{s})$ or "g local" (defined by Array.EarthGravity) and converted to absolute units using the value RawSeries.GtoGalConversionconstant.

Note that this distinction is implicit in the manner in which the Agency calibrated its instruments and processed its

data. In practice, many operators do calibrations by doing a flip test on site at the sensor's Geolocation, so that it is calibrated relative to Array.EarthGravity. These data typically are converted by g_standard. 
Similarly, if the RawSeries was converted from any unit,

such as "counts", to some other unit, <value> is that

(multiplicative) conversion constant and <units> are in

the sense <output units> / <input units>, for example,

(cm/s/s)/count to convert from a RawSeries in digital

counts to a Dataseries in gals.

\section{Table 3E(b). COSMOS and FDSN Array/Network Codes}

\begin{tabular}{|c|c|l|}
\hline $\begin{array}{c}\text { COSMOS } \\
\text { Code }\end{array}$ & $\begin{array}{c}\text { FDSN } \\
\text { Code }\end{array}$ & Network; Owner \\
\hline USCGS & - & U.S. Coast and Geodetic Survey \\
\hline NESMP & NP & $\begin{array}{l}\text { National Engineering Strong Motion Program (NESMP), U.S. Geological } \\
\text { Survey (USGS) }\end{array}$ \\
\hline USBR & RE & U.S. Bureau of Reclamation \\
\hline ACOE & - & U.S. Army Corps of Engineers \\
\hline $\begin{array}{c}\text { CDMG } \\
\text { or CGS }\end{array}$ & CE & $\begin{array}{l}\text { Calif. Geological Survey, formerly Div. of Mines and Geology } \\
\text { (CGS/California Strong Motion Instrumentation Program (CSMIP)) }\end{array}$ \\
\hline CIT & CI & Calif. Institute of Technology \\
\hline UCB & BK & $\begin{array}{l}\text { Berkeley Digital Seismic Network (BDSN); University of California, } \\
\text { Berkeley }\end{array}$ \\
\hline CWB & TW & Taiwan Central Weather Bureau (CWB) \\
\hline
\end{tabular}




\begin{tabular}{|c|c|c|}
\hline- & $\mathrm{AA}$ & $\begin{array}{l}\text { Anchorage Strong Motion Network; Geophysical Institute, Univ. of } \\
\text { Alaska, Fairbanks }\end{array}$ \\
\hline- & $\mathrm{AV}$ & Alaska Volcano Observatory; USGS, Anchorage \\
\hline- & $\mathrm{AZ}$ & $\begin{array}{l}\text { ANZA Regional Network; University of California, San Diego and } \\
\text { USGS, Menlo Park, Calif. }\end{array}$ \\
\hline- & BO & $\begin{array}{l}\text { NIED (Bosai-Ken Network); National Institute of Earth Science and } \\
\text { Disaster Prevention }\end{array}$ \\
\hline- & $\mathrm{CN}$ & Canadian National Seismic Network; Geological Survey of Canada \\
\hline- & $\mathrm{NC}$ & USGS Northern California Regional Network; USGS, Menlo Park, Calif. \\
\hline- & NN & Western Great Basin/Eastern Sierra Nevada; Univ. of Nevada, Reno \\
\hline- & $\mathrm{SN}$ & Southern Great Basin Network; Univ. of Nevada, Reno \\
\hline- & US & $\begin{array}{l}\text { U.S. National Seismic Network; ANSS backbone of USGS/NEIC, USGS/ASL } \\
\text { and EarthScope Project of IRIS }\end{array}$ \\
\hline- & UU & University of Utah Regional Network; University of Utah \\
\hline- & UW & Pacific Northwest net; University of Washington and USGS \\
\hline \multirow[t]{3}{*}{-} & WY & $\begin{array}{l}\text { Yellowstone Seismic Network; University of Utah (formerly by USGS, } \\
\text { Menlo Park) }\end{array}$ \\
\hline & ** & $\begin{array}{l}\text { All other FDSN/IRIS/SNCL network names are subsumed as acceptable } \\
\text { names here. }\end{array}$ \\
\hline & & $\begin{array}{l}\text { USER-EXTENSIBLE LIST (for networks not named above, use } \\
\text { established, terse, clear, English names or well-known } \\
\text { abbreviations which must begin with "User's description: ") }\end{array}$ \\
\hline
\end{tabular}

NOTES:

A complete set of these SNCL network abbreviations can be found at the following URLs.

For permanent networks: http://www.iris.washington.edu/stations/networks.txt.

For portable networks: http://www.iris.edu/stations/networks.portable.txt.

Array.Agency is REQUIRED unless the SNCL name GeoLocation.Name.SNCL or the SNCL-like name GeoLocation. Name.ExpandedSNCL is used. 


\section{Geographic-Position Issues}

Regarding the following, table $3 F$, we believe that two methods are necessary and sufficient for specifying the threedimensional position and of a GeoLocation and the orientation of a Sensor in space:

(1) Absolute coordinates and orientations (that is, locations within established geographic datums and orientations as azimuths from geographic true North and declinations from plumb vertical (that is, to the local gravity acceleration vector); or

(2) locations relative to a specified reference location and orientations relative to a reference orientation (such as the azimuth of a structure's long axis), with both references given in the absolute frames of (1).

Description method (2) typically would be used for structural and geotechnical Arrays and certain other compact Arrays. While it may be the case that the reference point and orientation are not precisely known, they should always be given, even when large corresponding error estimates must also be given. It is better to have a general idea where the Array is than no idea at all. (Of course, if "no idea at all" is all there is, give the value NULL.) It will often be the case in situations where absolute reference location and azimuth are poorly known that the offsets in location and orientation from these references will be well characterized (as in meters along, across, and up a structure and whether the sensor is wall-parallel or -perpendicular). Even if not, provide the available information and reasonable error estimates for same.

Both forms of Sensor location (for example, GeoLocation.Location.Latitude) are addressed in table 3F. Both forms of Sensor orientation are addressed in table 3K(a). There are more- and less-preferred methods for both, the less preferred methods provided for backward compatibility.

The first 13 tags of table 3F are the only place in which the absolute location of the Sensor is defined. The first five define the absolute location while the latter eight define an absolute (Array) reference location point plus the offsets from that point to the Sensor. Absolute locations typically are used in single-GeoLocation "Arrays" (typically free-field sites) while relative locations are typically used in structural and other Arrays containing GeoLocations distributed over some larger but relatively compact area or volume of observation. 
Of the CONDITIONAL tags among those first 13, at least one complete set must be selected, either the first three (absolute) or the latter six (relative).

\section{On the Accuracy of Geographic Positions}

If care is taken with conversions into VTF text and if IEEE double precision variables are used throughout in binary Processing, then in theory it is possible to store locations accurate to 15.65 digits (52-bit mantissa). (One would write out 16 to 17 digits to avoid round-off errors and maintain this degree of accuracy.) For Latitude, this precision equates to about $25 \mathrm{pm}$ (picometers), vastly better than is required. Geographic location precision of $10 \mathrm{~cm}$ (differential GPS accuracy) requires at least seven digits after the decimal for Latitude. Longitude values are similarly or more precise when given with the same number of digits after the decimal point.

\section{Table 3F. Other Geographic and Array Tags-Coordinates}

Absolute geographic position of the Sensor (there may be $(\boldsymbol{n})$ location attempts by one or more Agencies, including a history of small station relocations (that is, by a few meters to tens of meters, a common exigency to accommodate changing field conditions):

Please note that it is generally desirable to provide exactly one such Location, the one pertaining to the DataSeries in this VTF file (and, therefore, no explicit (n)). However, we provide the option of giving a history of GeoLocation movements and remeasurements.

If more than one GeoLocation.Location is given, put last (the highest index so "most recent" values) the authoritative values applicable to this DataSeries and be sure its DateTime is consistent with the DateTime of this VTF file (ThisFile.Preparation.DateTime and ThisFile.MetadataValid). 
Absolute geographic position of the proof mass:

GeoLocation.Location [ (m) ]. Latitude_dbl $=\langle$ value $\rangle$ deg;

|| Absolute, positive North, decimal degrees

GeoLocation.Location [ (m) ]. Longitude_dbl = <value> deg;

|| Absolute, positive East, decimal degrees

GeoLocation.Location [ (m) ].Elevation_dbl = <value $\rangle$ <units $>$;

Elevation above datum (meters preferred).

NOTE: While Sensor Elevations have been routinely

omitted from strong-motion site coordinates (these not generally being of interest to engineers), they are of

interest to seismologists and some research engineers.

Elevation should be given in all future data sets if

at all possible.

See ElevationofGradelevel (and

NumberofStories. [Above/Below]Grade) for all sensors

located away from grade level.

GeoLocation.Location [(m)].ElevationofGradeLevel_dbl = <value><units>; Depth of proof mass below surface (meters preferred).

REQUIRED if the sensor is more than one meter from grade level (as in a borehole or other underground location).

Also viable for above-grade installations, but these, in

general, should be supplied by using relative coordinates.

This tag is the only way, other than comments, to indicate a borehole or equivalent location. Use it with absolute or relative coordinates, or to give an explicit statement

of grade-level elevation.

GeoLocation.Location [ (m) ]. HorizontalDatum txt $=$ "<value $>$ " ;

|| Table 3G, "Horizontal" or "Mixed" datum

GeoLocation.Location [ (m) ] VerticalDatum txt $=$ "<value $\rangle$ ";

|| Table 3G, "Vertical" or "Mixed" datum

| | CONDITIONAL

| | CONDITIONAL

| | CONDITIONAL

| | CONDITIONAL
|| Recommended

|| Recommended 
GeoLocation.Location [(m)].HorizontalError dbl = <value $>$ <units ;

GeoLocation.Location [ (m) ].VerticalError_d $\bar{b} l=\langle$ value $\rangle<$ units $>$;

We note the possibility of representing an uncertainty

ellipsoid and seek comment on this matter.

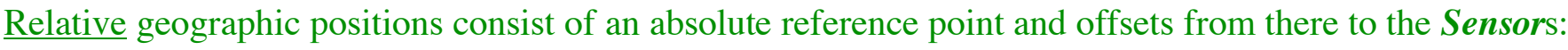

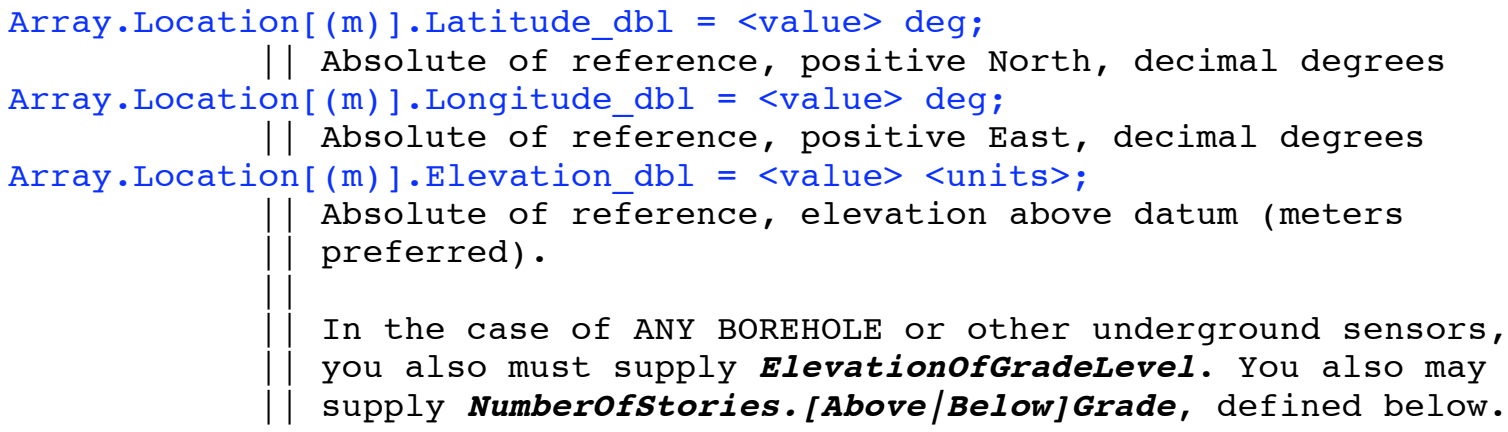

Array.Location [ (m) ].HorizontalDatum_txt $=$ "<value $>$ ";

|| Table 3G, "Horizontal" or "Mixed" datum

Array.Location [ (m)].VerticalDatum_txt = "<value $>$ ";

|| Table 3G, "Vertical" or "Mixed" datum

| | CONDITIONAL

| CONDITIONAL

| | CONDITIONAL

Array.Location [ (m) ].HorizontalError_dbl = <value $><$ units $>$;

Array.Location [ (m) ].VerticalError_dbl $=\langle$ value $\rangle\langle$ units $\rangle$;

( $\mathrm{km}$ preferred for <units>)

We note the possibility of representing an uncertainty

ellipsoid and seek comment on this matter. 
GeoLocation.Location [ (m) ]. Northoffset_dbl $=\langle$ value $><$ units $>$;

GeoLocation.Location [(m)].Eastoffset_d $\mathrm{dbl}=\langle\mathrm{value}\rangle\langle$ units $\rangle$;

GeoLocation.Location [(m)].Elevationoffset dbl = <value $\rangle$ <units

| North-, East-, and upward-positive offsets from the

Array.Location..., the reference location, for use in

Array-element positioning. (Meters are preferred

for all these units.)

GeoLocation.Location [(m)].WhichStory dbl = <value $>$;

|| Relates to GeoLocation.Building.NumberofStories...

indicating at which story of the structure the

sensor is to be found. The grade-level floor is

at story "0.0", upper floors have positive values,

and basements have negative values. Use of "dabl"

implies an option to specify partial stories, for

example, mid-column locations.

For all GeoLocations:

GeoLocation.Location [(m)]. Agency txt $=$ "<from table $3 \mathrm{E}(\mathrm{b})\rangle "$

Attribution of the above Geolocation coordinates

(either relative or absolute). Owner (Agency) of

set $(m)$ of the Geolocation.Location coordinates.

GeoLocation.Location [ (m) ].DateTime txt $=$

"YYYY-MM-DD [ hh:mm:ss [z|[+|-]hh[:mm]]]";

| Optional

Either the date ( \pm time) on which this Location was

determined, or the date ( \pm time) on which these data

were obtained from the Agency. If not given, this

DateTime may be assumed to be Processing.DateTime

or Thisfile.Preparation.DateTime, that is, either

the authoritative creation date of this Dataseries or

of this VTF file, with no clear preference. 
Owner/provider of this set of coordinates. Often the

same as Geolocation.Location.Agency, but REQUIRED

if an Array.Location is given.

Array.Location [(m) ].DateTime txt = "YYYY-MM-DD hh:mm:ss[z|[+|-]hh[:mm] ]"

|| Either the DateTime that this Location was determined,

| Optional

or the DateTime these data were obtained from the Agency.

If not given, assumed to be Processing.DateTime, the

authoritative creation date of this Dataseries.

Array.TotalRecorders_int $=\langle$ value $>$;

|| Number of recorders in the recording system of this Array. Array.Totalchannels int $=\langle$ value $\rangle$;

| Total number of channels in the recording system of this

Array. (Note also DAU.Totalchannels, needed because

there may be several DAUs in the Array. Similarly, the

tags Sensor.ArrayChannel and Sensor.DAUchannel are used

to identify this Dataseries' particular channel in the

Array and DAU.) 


\section{Table 3G. Geodetic Elevation Datums}

\begin{tabular}{|c|c|}
\hline Type of datum & Name of datum \\
\hline \multirow{3}{*}{ Horizontal } & “NAD83" \\
\hline & “NAD2 $7 "$ \\
\hline & "NZGD49" \\
\hline \multirow{3}{*}{ Mixed } & “WGS 72" \\
\hline & “WGS 84" \\
\hline & “ITRF00" \\
\hline \multirow{3}{*}{ Vertical } & "GEOID99" \\
\hline & “NAVD88" \\
\hline & “NGVD29" \\
\hline $\begin{array}{l}\text { USER-EXTENSIBLE LIST (use } \\
\text { established, terse, } \\
\text { clear, English names } \\
\text { which must begin with } \\
\text { "User's description: ") }\end{array}$ & $"$ \\
\hline
\end{tabular}

This list needs to be expanded to include, at least, the numerous datums used internationally, and perhaps those of historical interest. Full names and citations also would be useful additions.

Please use direct messages to the authors and/or the user-extensible-list option to help us make this list more complete. In particular, indicate the standard abbreviations and types of datums (horizontal, vertical, or both) for any or all of the following datums: 
Adindan; Afgooye; Ain_El_Abd_1970; Alaska_(NAD-27); Alaska/Canada_NAD-27; Anna_1_Astro_1965; ARC1950_mean; ARC-1960_mean; Ascension_Island_'58; Astro_B4_Sor.Atoll; Astro_Beacon_"E”; Astro_Pos_71/4; Astronomic_Stn._52; Australian_Geodetic_1984; Bahamas_(NAD-27); Bellevue_(IGN); Bermuda_1957; Bogota_Observatory; Bukit_Rimpah; Camp_Area_Astro; Campo_Inchauspe; Canada_Mean_(NAD27); Canal_Zone_(NAD27); Canton_Island_1966; Cape; Cape_Canaveral_mean; Carribean_(NAD27); Carthage; Central_America_(NAD27); Chatham_1971; Chua_Astro; Corrego_Alegre; Corrego_Alegre_(Provisional); Cuba_(NAD27); Cyprus; Djakarta(Batavia); DOS_1968; Easter_lsland_1967; Egypt; European_1950; European_1950_mean; European_1979_mean; Finnish_Nautical_Chart; Gandajika_Base; Geodetic_Datum_'49; Ghana; Greenland_(NAD27); Guam_1963; Gunung_Segara; Gunung_Serindung_1962; GUX_1_Astro; Herat_North; Hjorsey_1955; Hong_Kong_1963; Hu-Tzu-Shan; Indian; Iran; Ireland_1965; ISTS_073_Astro_'69; Johnston_Island_'61; Kandawala; Kerguelen_Island; Kertau_48; L.C._5_Astro; La_Reunion; Liberia_1964; Luzon; Mahe_1971; Marco_Astro; Masirah_Is._(Nahrwan); Massawa; Merchich; Mexico_(NAD27); Midway_Astro_'61; Mindanao; Minna; Montjong_Lowe; Nahrwan; Naparima_BWI; North_America_'83; North_America_1927_mean; Observatorio_1966; Old_Egyptian; Old_Hawaiian_mean; Old_Hawaiian_Kauai; Old_Hawaiian_Maui; Old_Hawaiian_Oahu; Oman; Ordnance_Survey_of_Great_Britain_36; Pico_De_Las_Nieves; Pitcairn_Astro_'67; Potsdam_Rauenberg_DHDN; Provisional_South_American_1956_mean; Provisional_South_Chilean_1963; Puerto_Rico; Pulkovo_1942; Qornoq; Quatar_National; Rome_1940; S_42; S.E.Asia_(Indian); SAD-69/Brazil; Santa_Braz; Santo_(DOS); Sapper_Hill_43; Schwarzeck; Sicily; Sierra_Leone_1960; South_American_1969_mean; South_Asia; Southeast_Base; Southwest_Base; Tananarive_Observatory_25; Thai/Viet_(Indian); Timbalai_1948; Tokyo_mean; Tristan_Astro_1968; Unites_Arab_Emirates_(Nahrwan); Viti_Levu_1916; Wake-Eniwetok_'60; WGS-72; WGS-84; Yacare; and Zanderij, (Dana, 1997). 


\section{Table 3H(a). Site Type and the Built Environment}

Geologic, geophysical, and geotechnical considerations:

There may be more than one determination, $(\boldsymbol{m})$, of the following values. Further, the sense of "Recommended" here is "strongly recommended" for at least some subset of these site-condition characterizations.

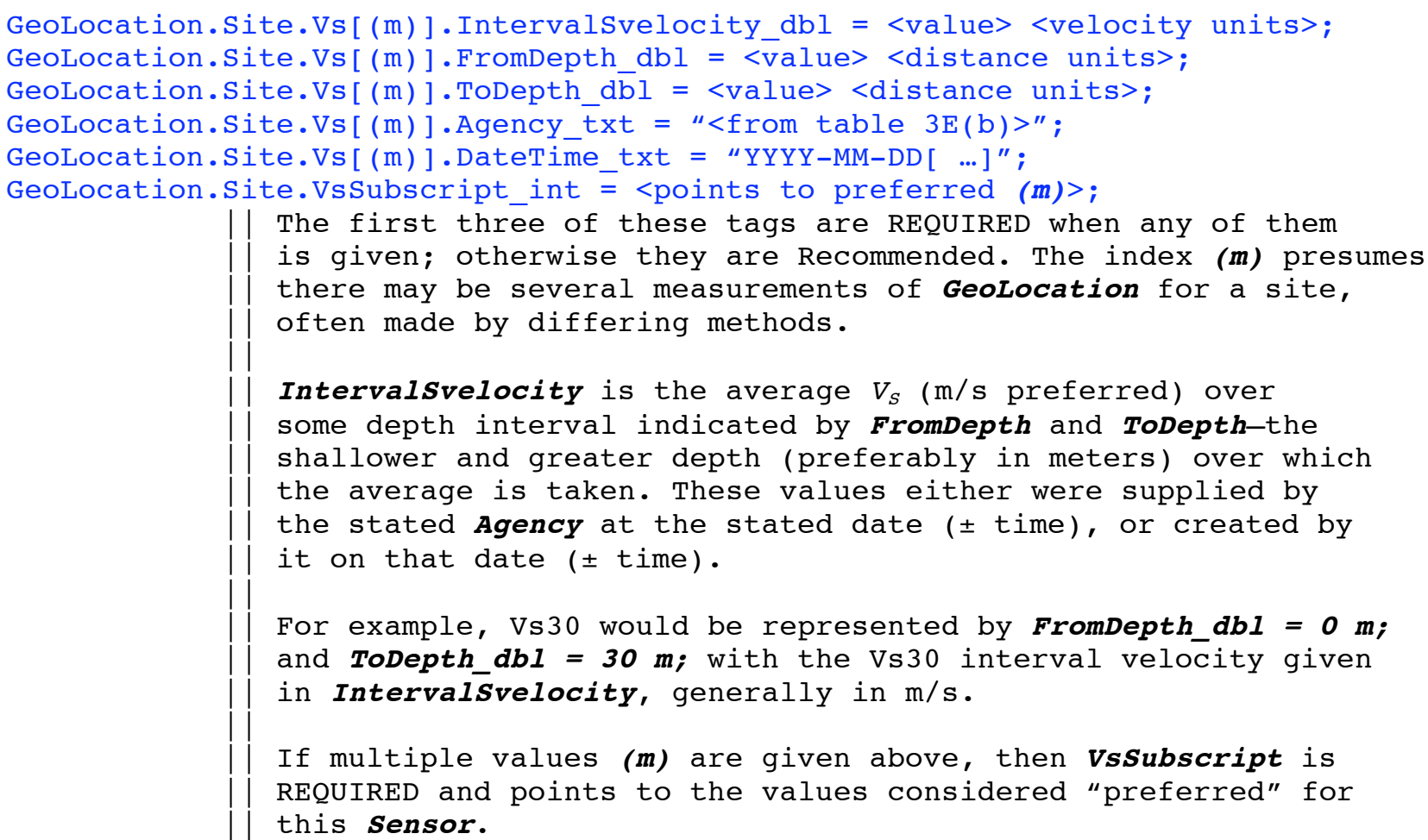


|l or the date ( \pm time) the code was obtained from the Agency.

GeoLocation.Site.Code[(m)]. Citation_txt = "<description of codes $>$ ";

GeoLocation.Site.Code [(m)].URL_txt = "<Web address $>$ ".

| If possible, cite a description the type of the site.Code used here.

Geolocation.Site.Code[(m)].TextValue_txt = "<the site-condition code>"; Any site-condition cōede developed by any organization or individual. The values for Agency may be those listed in table 3E, or any other "owner" of these codes, but not necessarily the organization that developed the code.

For example, for NEHRP Vs30 site codes:

\begin{tabular}{|c|c|}
\hline NEHRP Code & VS30 Range (m/s) \\
\hline A & $>1500$ \\
\hline B & 760 to 1500 \\
\hline C & 360 to 760 \\
\hline$D$ & 180 to 360 \\
\hline E & $<180$ \\
\hline
\end{tabular}

the values generally would be

Geolocation.Site.Code(n).Agency_txt = "<DataSeries network code $>$ ";

Geolocation.Site.Code(n).DateTime_txt = "<Year-Month-Day $\rangle$ ";

Geolocation.Site.Code(n).Citation_txt = "NEHRP (revised)";

Geolocation.Site.Code(n).TextValue_txt = [ "A" | $\mathrm{B}$ " | "C" $\mid$ "D" | $\mathrm{E}$ " ];

Other examples of codes are the GeoMatrix three-character code, Campbell's GEOCODE, UBC site class codes (Wills and others, 2000 , and their subsequent work), and Bray and Rodriquez-Marek's (1997, and their subsequent work) site codes. It is likely that there are many others. 
which (m) is "preferred" above. REQUIRED if more

than one index is given, but may be NULL to indicate

"no stated preference" or "unknown".

GeoLocation.Site.Isopleth.ShearVelocity_dbl = <velocity $\rangle<$ units $>$;

GeoLocation.Site.IsoPleth. Depth dbl $=\langle$ depth $\rangle<$ units $>$;

CONDITIONAL

CONDITIONAL

|| The depth to a particular velocity horizon at

the site, and the wave speed at said isopleth.

If Depth is given, so must be shearvelocity.

GeoLocation.Site.Bedrock. Depth dbl = <value $><$ units $>$;

GeoLocation.Site.Bedrock.Comments_txt = "<Comments \pm unit name $>$ ";

Depth to bedrock. For example, Depth in kilometers to

the Franciscan formation. Comments provides the means

to describe what this bedrock is, typically a unit name

and "[ top | bottom ] of ...". (It is OK to give only

Comments naming the bedrock unit since sometimes this

is known but depth is not. In this case, it may be

wise to include a general Basin description, too.)

If either of these tags is given, then both are

RECOMMENDED.

GeoLocation.Site.Basin.Depth.Description txt = [ "Shallow" |"Deep" ];

GeoLocation.Site.Basin. Depth.Value_dbl = $<$ value $><$ units $>$;

GeoLocation.Site.Basin.Depth.Error_dbl = <value $\rangle\langle$ units $\rangle$;

GeoLocation.Site.Basin. Name txt = "<basin"s name>";

GeoLocation.Site.Basin.Citation_txt = " $<$ Citation $>$ ";

GeoLocation.Site.Basin.Comments_txt = "<Comments $>$ "; 
The depth, at the Sensor's Geolocation, of a sedimentary and/or volcaniclastic basin or any surface low-velocity unit having a similar effect on the data. When a numeric Depthvalue is unknown but a general sense of the basin is available, GeneralDepth is this general assessment of Basin depth, for example, as used by Bray and Rodriquez-Marek (1997), who separate "Shallow" from "Deep" at 60-m depth. In contrast, Depthvalue is an explicit depth estimate (for example, in meters). Name, Citation, and Comments identify the Basin, to which the information applies, as well as any present issues.

CAUTION: At some point, some sort of BasinEdge information also should be defined as for the above six classes, describing distances from the Geolocation or the Event.Hypocenter to the edges of Hypocenter relevant Basins, and perhaps the detailed geography of the Basin, but this is a complex and potentially expansive issue not yet addressed.

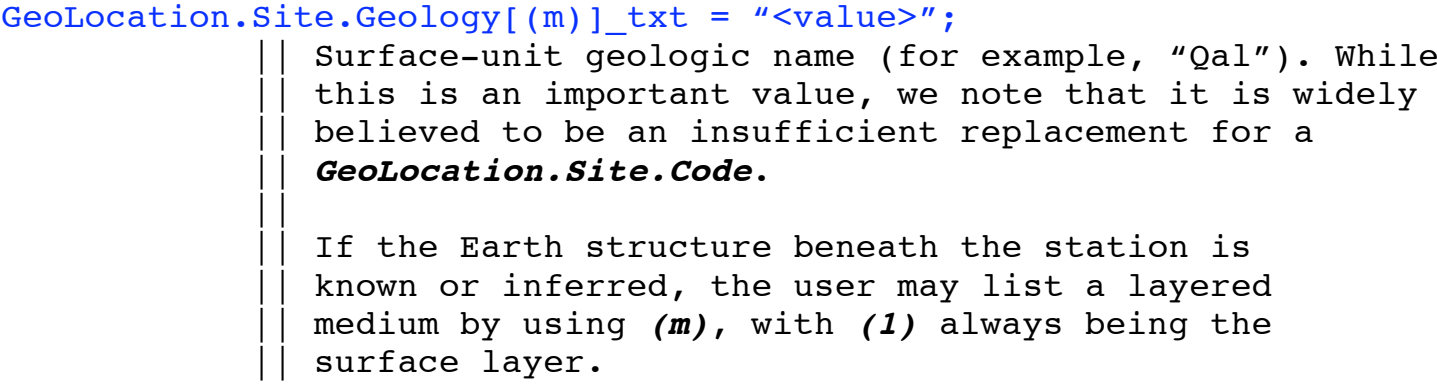

If the Earth structure beneath the station is known or inferred, the user may list a layered medium by using (m), with (1) always being the surface layer.

GeoLocation.Site.SurfaceAge txt = "<value $>$ ";

|| Age or epoch of the surface geologic unit. For example, "Holocene" or "8000 years".

GeoLocation.Site.SurfaceGrainsize_txt = [ "Aggregate" | "Coarse" | "Fine" ]; || Grain size of the surface geologic unit 
GeoLocation.Site.Citation_txt = "<Citation $>$ " ;

GeoLocation.Site.URL_txt = "<Web address $>$ ";
A citation or pointer to a provider's detailed site
description (geology, geophysics, geotechnical,
structures, and any other site information
affecting response).

GeoLocation.Site.Geotechnicalfeature [ (m) ]. Identifier txt = "<value $>$ ";

GeoLocation.Site.Geotechnicalfeature [ (m) ]. Citation_t $\bar{x} t=$ "<value $>$ ";

GeoLocation.Site.GeotechnicalFeature [(m)].URL_txt = "<Web address $>$ ";

GeoLocation.Site.Geotechnicalfeature [(m)]. Distance dbl = <value $\rangle$ <units $>$;

GeoLocation.Site.Geotechnicalfeature [(m) ]. Type_txt ${ }^{-}=$"<value $>$" ;

GeoLocation.Site.GeotechnicalFeature [ (m) ].Distributor txt =

$$
\text { [ "GVDC" "<other value>" ]; }
$$

GeoLocation.Site.GeotechnicalFeature [ (m) ].Agency_txt = "<value $>$ ";

The cosmos Geotechnical Virtual Data Center (GVDC) will soon

become a leading means of reaching large stores of detailed

geotechnical data. The tags here anticipate the "unique

Identifier" of features in that virtual data base.

Additionally, these tags are general enough to reference

geotechnical data from any source, either by using the

Identifier, Citation, or URL, or by using data from an original

source Agency often through a Distributor, such as the GVDC.

These features are likely to include boreholes, CPT soundings, trench logs, SASW and other seismic profiles, and so forth.

Distance provides the distance from the Geolocation of this Dataseries measurement to the location of the geotechnical

feature. Similarly, Type names the type of feature, preferably

by using the names of GVDC's xml structures identifying the

feature (for example, "hole" for boreholes). 
The built environment versus reference and free field:

One set of the following CONDITIONAL tags is REQUIRED to properly describe the siting conditions of this individual Sensor at this specific GeoLocation.

We note that it might be thought that these should be Array tags, however, different GeoLocations within an Array of instruments can be structural, reference or free-field, and in different settings within a structure (abutment, mid-span, and so forth). For example, consider the San Francisco Bay Bridge, which has abutments on hard rock and floating in deep Bay mud, has reference sites in boreholes and on the surface of both environments, and has Sensors in numerous settings along the 10-km-long structure, on steel or concrete, old and new.

Therefore, the description must be specific to each GeoLocation within the Array, specific to the single record represented in this file. While current tags do not capture the full complexity of possible descriptions, the way is opened for complete descriptions, and the process is at least brought to the point where distinctions can be made between reference sites and those on the structure.

Note that tags are grouped and a logical set of related tags is required to complete this section. If the GeoLocation is in reference of free-field conditions, then only the first tag is needed. If it is a built setting, one of the structure sets of tags is required.

Note that where an index $(\boldsymbol{m})$ appears below, it is being used in most cases to indicate that some structure varies significantly from one area to another, or that there are several, adjacent or nearly adjacent structures, with these parts possibly performing differently or interacting in some way (for example, they may have contrasting mode periods, or there might be collisions between them, or the grouping creates a "many body" soil-structure interaction). In this case, it is preferable also to include the corresponding <TypeOfStructure $>$ Subscript tag to tell the user which description applies best to this specific GeoLocation.Location (coordinates). 
Between the REQUIRED GeoLocation.StructureInfluence, and the set of CONDITIONAL tags (NonStructuralDeploymentDescription, OtheStructure.Description, GeoLocation.Building...., GeoLocation.Bridge...., and GeoLocation.Dam....) at least a basic description of Sensor siting conditions is REQUIRED (a sufficient set of these tags).

One tag that applies to all GeoLocations, all Sensors:

GeoLocation.StructureInfluence txt $=$

$$
\text { [ "Free field" | "Reference" | "In or on structure" ]; }
$$
Select which of the three strings best describes the degree to which the built-environment (structures) impact the response of this Sensor-in effect, no influence, possibly some influence, or significant influence. Please give preference to using the COSMOS/ANSS standards in making this distinction.

Descriptors that apply to all non-structural GeoLocations and Sensors:

GeoLocation.NonStructuralDeploymentDescription [(m)]_txt = "<Terse Description>" || One or more text description(s) from table $3 \mathrm{H}(\mathrm{b})$ which describe the installation details of either a free-field or a reference Sensor at this Geolocation.

This tag is used only for nonstructural installations. For sensors in/on structures, use a set of the tags below.

It is assumed that all (m) pertain simultaneously and equally. 
Descriptors that apply to all structural GeoLocations and Sensors:

GeoLocation.StructurePedigree.DateApplied.DateTime_txt = "YYYY-MM-DD [ ...]" ; GeoLocation.StructurePedigree.DateApplied.TimeError dbl =

$$
\text { <value> [ years | months | days | ... ] ; }
$$

GeoLocation.StructurePedigree.DesignCodeUsed[(m)]_txt = "<Building Code(s) Used $>$ "

The estimated or actual DateTime of design or construction,

generally to the nearest year only, thus specifying the year

controlling structural qualities. That is, the inception,

completion, or other controlling date ( \pm time), of the

structure and the uncertainty in this design-controlling

DateTime.

Also, the building code or codes $(\boldsymbol{m})$ under which this

structure was designed and built. For example, "2006 IBC".

Descriptors that apply only to buildings:

GeoLocation.Building[(m)].StructuralSystem[(n)] txt $=$ "<Terse Description>". || Recommended when the Geolocation is in or on a building. Use one or more descriptions from table $3 \mathrm{H}(\mathrm{c})$.

(If a code from table $3 \mathrm{H}(\mathrm{e}$ or $\mathrm{f}$ ) is desired, instead use GeoLocation.Building.HAZUSoccupancyClass and/or Geolocation.Building.HAZUSbuildingType.)

Use tags enumerated elsewhere below when the structure is not a building (is a Bridge, Dam, or otherstructure).

It is assumed that all (n) pertain equally.

GeoLocation.Building [(m) ]. HAZUSbuildingType txt $="<$ From table $3 \mathrm{H}(\mathrm{e})>$ "; GeoLocation.Building $[(\mathrm{m})]$. HAZUSoccupancyClass_txt $=$ "<From table $3 \mathrm{H}(\mathrm{f})>$ ";

|| Two HAZUS codes indicating the type of building, and its

in addition to other descriptors. 
GeoLocation.Building [(m) ].NumberOfStories.AboveGrade_int $=\langle$ value $>$; GeoLocation. Building [ (m) ]. NumberOfStories.BelowGrade int $=\langle$ value $\rangle$;

Recommended if the structure is a building. Pairs with

Geolocation.Building.Structuralsystem(m) to form a

complete description. Can be subscripted (m) if the

building is complex, with sections of differing height

or structural type.

Note the relationship to Geolocation.Location. Whichstory, and with ElevationofGradelevel.

GeoLocation.Building[(m)].Height_txt = "[ Low | Medium | High ] Rise"; | Recommended if the structure is a building and the

number of stories is not known precisely. Pairs with

Geolocation.Building.Structuralsystem(n) to form

a complete description.

CSMIP usage is

"L" = "Low Rise" = 1 to 3 stories

"M" = "Medium Rise" = 4 to 7 stories

"H" = "High Rise" = 8 or more stories.

and is a recommended usage.

Exactly one Geolocation.Building.NumberofStories

or Geolocation.Building.Height is REQUIRED if the

Geolocation is in or on a building.

GeoLocation.Building $[(\mathrm{m})]$. Description_txt $=$ "<Terse Description $>$ ";

A brief description of an instrumented building not

elsewhere described or describable. If you need

more than one line of text, use subscripts (m), or

put the entire description into one long string with

"lines" separated by the character string "/|n|".

| | CONDITIONAL

GeoLocation. BuildingSubscript_int $=<$ value of $(m)$ where this Sensor is $>$;

If there are more than one (m) above, then this tag is

REQUIRED and points to the Geolocation.Building [(m) ]...

pertaining specifically to this Sensor. 
Descriptors that apply only to bridges:

GeoLocation.Bridge[(m)].Description_txt = "<Terse English Description>"; Brief description of an instrumented bridge not

elsewhere described or describable. If the Bridge

has more than one section, use subscript (m).

For multiple lines in one description use one, long string

with "lines" separated by the character string "/|n/".

GeoLocation.Bridge $[(\mathrm{m})]$. HAZUSoccupancyClass_txt $="<$ From table $3 \mathrm{H}(\mathrm{f})>$ ";

A HAZUS code indicating the occupancy pattern of this

bridge. Tag may be given in place of or in addition to

other descriptors.

We note that HAZUS does not currently list bridge-

occupancy descriptors for vehicular lifelines so

this is a placeholder at present.

GeoLocation.Bridge [ (m) ]. SubstructureMaterial_txt $=$ "<from table $3 \mathrm{H}(\mathrm{d})\rangle$ ";

GeoLocation.Bridge [ (m) ]. SuperstructureConfiguration_txt $=$ "<from table $3 \mathrm{H}(\mathrm{d})>$ ";

GeoLocation.Bridge $[(\mathrm{m})]$. NumberOfSpans txt $="<$ from table $3 \mathrm{H}(\mathrm{d})>"$ "

GeoLocation.Bridge [ (m) ] . AbutmentType_txt $=$ "<from table $3 \mathrm{H}(\mathrm{d})\rangle$ ";

GeoLocation.Bridge [(m)].SpanContinuity_txt $="\langle$ from table $3 \mathrm{H}(\mathrm{d})\rangle "$;

GeoLocation.Bridge [(m) ]. ColumnsPerBent_txt $=$ " $<$ from table $3 \mathrm{H}(\mathrm{d})\rangle$ ";

|| If the Geolocation structure type is a bridge and the

| | CONDITIONAL

information is available, then use these six values

| preferentially to classify the structure according to the

|| descriptive vectors of Basöz and Kiremidjian (1995, 1996).

|| (If there are strongly contrasting sections of the bridge,

these may be enumerated by subscripting (m), as with the

East and West sections of the San Francisco Bay Bridge.)

The following are the equivalent vector-element names of

Basöz and Kiremidjian (1996): 


\section{yln: Geolocation.Bridge.SubstructureMaterial}

y2n: Geolocation.Bridge.SuperstructureConfiguration

y31: GeoLocation.Bridge.NumberofSpans

y32: Geolocation.Bridge.AbutmentType

y33: Geolocation.Bridge.SpanContinuity

y34: Geolocation.Bridge.ColumnsPerBent

If any one is given, the first four (for single-span bridges), or all six (for multiple-span bridges), should be included. However, for situations where not all are available, subsets are allowed and may be mixed with Geolocation.Bridge.Description.

Note that a few combinations of substructurematerial and superstructureconfiguration are believed not to exist, at least in California (see reference). Weighted combinations of values also are possible, but are not treated here (yet) except by the option to use subscripting (m) to describe separately several major sections of the bridge. Possible values are listed in table $3 \mathrm{H}(\mathrm{d})$.

GeoLocation.BridgeSubscript int $=<$ value of $(m)$ where this sensor is $>$; If there are more than one (m) above, then this tag is REQUIRED and points to the GeoLocation.Bridge(m)... that pertains specifically to this Geolocation, this Sensor.

Descriptors that apply only to $\underline{\text { dams: }}$

GeoLocation.Dam[(m)].Description_txt $=$ "<Terse Description $>$ "; Brief description of the dam from table $3 \mathrm{H}(\mathrm{c})$, or a brief description of an instrumented dam not elsewhere described or describable. If there is more that one, structurally unique section of the Dam, use subscripts (m) to indicate these different parts. For a particular section of the Dam needing multiple descriptors put it all into one string with "lines" separated by the character string "/|n/". 
Noting that the descriptions in table $3 \mathrm{H}(\mathrm{b})$ are very general, we seek a more detailed description of Dams. This descriptive technique should be clear, concise, fairly complete, and have at least some degree of acceptance by, and standardization within, the engineering community. Please direct suggestions to the authors.

GeoLocation.DamSubscript int $=<$ value of $(m)$ where this sensor is $>$;

If there are more than one $(m)$ in what follows, this tag is

REQUIRED and points to the GeoLocation.Dam(m) pertaining

specifically to this Geolocation, this Sensor.

Descriptors that apply to any structure not provided for elsewhere above:

GeoLocation.OtherStructure[(m)].Description_txt $=$ "<Terse Description $>$ ";

A brief description of an instrumented structure not

| CONDITIONAL

A

table $3 \mathrm{H}(\mathrm{c})$, which is a USER-EXTENSIBLE list).

If there is more than one contrasting section of the structure, use subscripts, (m).

For any particular section of the structure put it all

into one long string with "lines" separated by the

character string "/ $\mathbf{n} /$ ".

GeoLocation. OtherStructure [ (m)].HAZUSoccupancyclass_txt $=$ "<From table $3 \mathrm{H}(\mathrm{f})>$ ";

A HAZUS code indicating the occupancy pattern of the

structure. May be given in place of, or in addition

to, other descriptors.

GeoLocation. OtherStructureSubscript_int $=<$ value of $(m)$ where this sensor is $>$;

If there are more than one $(\boldsymbol{m})$, more than one contrasting

section of the structure, then this tag is REQUIRED and

points to the (m) pertaining specifically to this sensor.

|| CONDITIONAL 


\section{Table 3H(b). Siting Conditions, Reference, and Free Field}

\begin{tabular}{|c|c|}
\hline Name & Description [or additional information] \\
\hline \multicolumn{2}{|c|}{$\begin{array}{l}\text { Free field, ground response, or reference sites } \\
\text { (GeoLocation.NonstructuralDeploymentDescription(m)): }\end{array}$} \\
\hline "Small fiberglass shelter" & $\begin{array}{l}\text { Small, prefabricated, fiberglass shelter (typically } \\
1-2 \mathrm{~m} \text { on each dimension). }\end{array}$ \\
\hline "Small metal shelter" & $\begin{array}{l}\text { Small, prefabricated, metal building (typically } 1-2 \mathrm{~m} \\
\text { on each dimension). }\end{array}$ \\
\hline "Small wooden shelter" & $\begin{array}{l}\text { Small, prefabricated or place-built, wood or wood- } \\
\text { frame structure (typically } 1-2 \mathrm{~m} \text { on each dimension). }\end{array}$ \\
\hline "Shallow" & Sensors buried/set in ground (shallow, near surface). \\
\hline Nota Bene & $\begin{array}{l}\text { In the case of reference sites in light or mid-weight } \\
\text { buildings, use the apropos "Building Type" listing } \\
\text { below, but append the text "||n|REFERENCE" in } \\
\text { capitals to any other descriptors. }\end{array}$ \\
\hline "Other Free Field" & Unspecified or unknown free-field site. \\
\hline "Other Reference" & Unspecified or unknown reference site. \\
\hline \multicolumn{2}{|c|}{ Array types (GeoLocation.NonStructuralDeploymentDescription $(m)$ ) : } \\
\hline "Geotechnical Array" & Geotechnical Array \\
\hline "Dense Array" & Array with mean spacing of 1.0 to $3.0 \mathrm{~km}$ \\
\hline "Very Dense Array" & Array with mean spacing of 0.3 to $1.0 \mathrm{~km}$ \\
\hline "Ultra-dense Array" & Array with mean spacing of $<0.3 \mathrm{~km}$ \\
\hline "Other Array" & Unspecified Array \\
\hline If all else fails: & \\
\hline “ & $\begin{array}{l}\text { USER-EXTENSIBLE LIST (use established, terse, clear, } \\
\text { English names or phrases OR tersely cite a paper } \\
\text { which must begin with "User's description: "). Try to } \\
\text { keep well below } 60 \text { characters. }\end{array}$ \\
\hline
\end{tabular}




\section{Table 3H(c). Built Environment Values (CSMIP)}

\begin{tabular}{|c|c|}
\hline $\begin{array}{l}\text { Building structural system } \\
\text { (Geolocation.Building.structuralsystem }(m) \text { ) }\end{array}$ & Append one of these Building Heights ** \\
\hline "Unreinforced Masonry Bearing Wall" & \multirow{20}{*}{$\begin{array}{l}\text { (This appended text value is now replaced } \\
\text { by Geolocation.Building.Height) }\end{array}$} \\
\hline “Unreinforced Masonry with Load Bearing Frame” & \\
\hline "Non-ductile Concrete Moment Frame" & \\
\hline "Concrete Tilt-up" & \\
\hline “Reinforced Masonry Wall without Moment Frame” & \\
\hline "Concrete Shear Wall without Moment Frame" & \\
\hline $\begin{array}{l}\text { "Reinforced Masonry Shear Wall without Moment } \\
\text { Frame" }\end{array}$ & \\
\hline "Concrete Shear Wall and Moment Frame" & \\
\hline "Steel Braced Concrete Frame" & \\
\hline "Precast Concrete (Not Tilt-up)" & \\
\hline “Precast Reinforced Concrete (Not Tilt-up)" & \\
\hline "Braced Steel Frame" & \\
\hline "Reinforced Masonry Shear Wall and Moment Frame" & \\
\hline "Ductile Concrete Moment Frame" & \\
\hline "Moment Resisting Perimeter Steel Frame" & \\
\hline "Wood Frame" & \\
\hline "Base Isolated" & \\
\hline “Hospitals since 1989 (OSHPD)" & \\
\hline "Retrofitted" & \\
\hline "Other Building Type" & \\
\hline
\end{tabular}




\begin{tabular}{|c|c|}
\hline \multicolumn{2}{|c|}{ Dams (Geolocation.Dam.Description): } \\
\hline "Concrete Gravity Dam" & Concrete gravity dam \\
\hline "Concrete Arch Dam" & Concrete arch dam \\
\hline “Engineered Fill Dam" & Engineered fill dam \\
\hline "Hydraulic Fill Dam" & Hydraulic fill dam \\
\hline “Unknown-Fill Dam” & An earth-fill dam of uncertain type \\
\hline "Small Unengineered Dam" & $\begin{array}{l}\text { Small unengineered dam (for example, cattle } \\
\text { pond) }\end{array}$ \\
\hline “Rock Dam” & Rock dam with impervious core \\
\hline "Other Dam" & Unspecified dam \\
\hline \multicolumn{2}{|c|}{$\begin{array}{l}\text { Other Structures (GeoLocation.otherstructure.Description }(m) \text { or } \\
\text { Geolocation.Building.Structuralsystem }(m)):\end{array}$} \\
\hline "Parking Structure" & Parking structure \\
\hline "Stadium" & Sports or performance open-air structure \\
\hline "Other Building" & Unspecified building \\
\hline "Other structure" & Unspecified structure \\
\hline " & $\begin{array}{l}\text { USER-EXTENSIBLE LIST (give a terse, clear, } \\
\text { English description, which must begin with } \\
\text { "User's description: "). }\end{array}$ \\
\hline
\end{tabular}

**For the CSMIP building-type listing, the appended notations: "L" = Low Rise (1 to 3 stories); "M" = Medium Rise (4 to 7 stories); "H" = High Rise (8 or more stories) is ; "OSHPD" = Office of Statewide Health Planning and Development. (Shakal and others 2002).

NOTE: The specific (maximum) number of a building's stories above and/or below grade may be given explicitly in GeoLocation.Building.NumberOfStories, or as a general range in GeoLocation.Building.Height. 


\section{Table 3H(d). Siting Conditions, Bridges}

\begin{tabular}{|c|c|}
\hline \multicolumn{2}{|c|}{ Bridge values (Basöz and Kiremidjian, 1995, 1996): } \\
\hline "Concrete" & y11, Geolocation.Bridge.SubstructureMaterial \\
\hline "Steel" & y12, GeoLocation.Bridge.SubstructureMaterial \\
\hline "Timber" & y13, GeoLocation.Bridge.SubstructureMaterial \\
\hline "Masonry" & y14, Geolocation.Bridge.SubstructureMaterial \\
\hline "Concrete Girder" & y21, GeoLocation.Bridge.SuperstructureConfiguration \\
\hline "Steel Girder" & y22, GeoLocation.Bridge.SuperstructureConfiguration \\
\hline "Steel Truss" & y23, GeoLocation.Bridge.SuperstructureConfiguration \\
\hline "Suspension" & y24, GeoLocation.Bridge.SuperstructureConfiguration \\
\hline "Arch" & y25, GeoLocation.Bridge.SuperstructureConfiguration \\
\hline "Single" & \multirow{3}{*}{ y31, GeoLocation.Bridge.Numberofspans } \\
\hline "Multiple" & \\
\hline "<number>" & \\
\hline "Monolithic" & \multirow{2}{*}{ y32, GeoLocation.Bridge.AbutmentType } \\
\hline "Not Monolithic" & \\
\hline "Continuous" & \multirow{2}{*}{ y33, GeoLocation.Bridge.SpanContinuity } \\
\hline "Discontinuous" & \\
\hline "Single" & \multirow{3}{*}{ y34, GeoLocation.Bridge.ColumnsPerBent } \\
\hline "Multiple" & \\
\hline "<number>" & \\
\hline \multicolumn{2}{|c|}{ Bridge values for Geolocation.Bridge.Description only } \\
\hline "Other Bridge" & Unspecified bridge \\
\hline
\end{tabular}




\section{Table 3H(e). Site Type and Built Environment Values-HAZUS Codes}

\begin{tabular}{|c|c|c|c|c|c|c|}
\hline \multirow{3}{*}{$\begin{array}{c}\text { No } \\
\text { - }\end{array}$} & \multirow{3}{*}{$\begin{array}{c}\text { HAzUS } \\
\text { building } \\
\text { type }\end{array}$} & \multirow{3}{*}{ Description } & \multicolumn{4}{|c|}{ Height } \\
\hline & & & \multicolumn{2}{|c|}{ Range } & \multicolumn{2}{|c|}{ Typical } \\
\hline & & & Name & Stories & Stories & Feet \\
\hline 1 & W1 & Wood, Light Frame $(\leq 5,000 \mathrm{sq}$. ft. $)$ & & $1-2$ & 1 & 14 \\
\hline 2 & W2 & $\begin{array}{l}\text { Wood, Commercial and Industrial } \\
(>5,000 \mathrm{sq} \text {. ft. })\end{array}$ & & All & 2 & 24 \\
\hline 3 & S1L & \multirow{3}{*}{ Steel Moment Frame } & Low-Rise & $1-3$ & 2 & 24 \\
\hline 4 & $\mathrm{~S} 1 \mathrm{M}$ & & Mid-Rise & $4-7$ & 5 & 60 \\
\hline 5 & $\mathrm{~S} 1 \mathrm{H}$ & & $\begin{array}{c}\text { High- } \\
\text { Rise }\end{array}$ & $8+$ & 13 & 156 \\
\hline 6 & S2L & \multirow{3}{*}{ Steel Braced Frame } & Low-Rise & $1-3$ & 2 & 24 \\
\hline 7 & $\mathrm{~S} 2 \mathrm{M}$ & & Mid-Rise & $4-7$ & 5 & 60 \\
\hline 8 & $\mathrm{~S} 2 \mathrm{H}$ & & $\begin{array}{c}\text { High- } \\
\text { Rise }\end{array}$ & $8+$ & 13 & 156 \\
\hline 9 & S3 & Steel Light Frame & & All & 1 & 15 \\
\hline 10 & $\mathrm{~S} 4 \mathrm{~L}$ & \multirow{3}{*}{$\begin{array}{c}\text { Steel Frame with Cast-in-Place } \\
\text { Concrete Shear Walls }\end{array}$} & Low-Rise & $1-3$ & 2 & 24 \\
\hline 11 & $\mathrm{~S} 4 \mathrm{M}$ & & Mid-Rise & $4-7$ & 5 & 60 \\
\hline 12 & $\mathrm{~S} 4 \mathrm{H}$ & & $\begin{array}{c}\text { High- } \\
\text { Rise }\end{array}$ & $8+$ & 13 & 156 \\
\hline 13 & S5L & \multirow{3}{*}{$\begin{array}{c}\text { Steel Frame with Unreinforced } \\
\text { Masonry Infill Walls }\end{array}$} & Low-Rise & $1-3$ & 2 & 24 \\
\hline 14 & S5M & & Mid-Rise & $4-7$ & 5 & 60 \\
\hline 15 & $\mathrm{~S} 5 \mathrm{H}$ & & $\begin{array}{l}\text { High- } \\
\text { Rise }\end{array}$ & $8+$ & 13 & 156 \\
\hline 16 & $\mathrm{C} 1 \mathrm{~L}$ & \multirow{3}{*}{ Concrete Moment Frame } & Low-Rise & $1-3$ & 2 & 20 \\
\hline 17 & $\mathrm{C} 1 \mathrm{M}$ & & Mid-Rise & $4-7$ & 5 & 50 \\
\hline 18 & $\mathrm{C} 1 \mathrm{H}$ & & $\begin{array}{c}\text { High- } \\
\text { Rise }\end{array}$ & $8+$ & 12 & 120 \\
\hline
\end{tabular}




\begin{tabular}{|c|c|c|c|c|c|c|}
\hline 19 & $\mathrm{C} 2 \mathrm{~L}$ & \multirow{3}{*}{ Concrete Shear Walls } & Low-Rise & $1-3$ & 2 & 20 \\
\hline 20 & $\mathrm{C} 2 \mathrm{M}$ & & Mid-Rise & $4-7$ & 5 & 50 \\
\hline 21 & $\mathrm{C} 2 \mathrm{H}$ & & $\begin{array}{l}\text { High- } \\
\text { Rise }\end{array}$ & $8+$ & 12 & 120 \\
\hline 22 & C3L & \multirow{3}{*}{$\begin{array}{c}\text { Concrete Frame with Unreinforced } \\
\text { Masonry Infill Walls }\end{array}$} & Low-Rise & $1-3$ & 2 & 20 \\
\hline 23 & $\mathrm{C} 3 \mathrm{M}$ & & Mid-Rise & $4-7$ & 5 & 50 \\
\hline 24 & $\mathrm{C} 3 \mathrm{H}$ & & $\begin{array}{l}\text { High- } \\
\text { Rise }\end{array}$ & $8+$ & 12 & 120 \\
\hline 25 & $\mathrm{PC} 1$ & Precast Concrete Tilt-Up Walls & & All & 1 & 15 \\
\hline 26 & PC2L & \multirow{3}{*}{$\begin{array}{l}\text { Precast Concrete Frames with } \\
\text { Concrete Shear Walls }\end{array}$} & Low-Rise & $1-3$ & 2 & 20 \\
\hline 27 & PC2M & & Mid-Rise & $4-7$ & 5 & 50 \\
\hline 28 & $\mathrm{PC} 2 \mathrm{H}$ & & $\begin{array}{l}\text { High- } \\
\text { Rise }\end{array}$ & $8+$ & 12 & 120 \\
\hline 29 & RM1L & \multirow{2}{*}{$\begin{array}{l}\text { Reinforced Masonry Bearing Walls with } \\
\text { Wood or Metal Deck Diaphragms }\end{array}$} & Low-Rise & $1-3$ & 2 & 20 \\
\hline 30 & RM1M & & Mid-Rise & $4+$ & 5 & 50 \\
\hline 31 & RM2L & \multirow{3}{*}{$\begin{array}{l}\text { Reinforced Masonry Bearing Walls } \\
\text { with Precast Concrete Diaphragms }\end{array}$} & Low-Rise & $1-3$ & 2 & 20 \\
\hline 32 & RM2M & & Mid-Rise & $4-7$ & 5 & 50 \\
\hline 33 & $\mathrm{RM} 2 \mathrm{H}$ & & $\begin{array}{l}\text { High- } \\
\text { Rise }\end{array}$ & $8+$ & 12 & 120 \\
\hline 34 & URML & \multirow{2}{*}{ Unreinforced Masonry Bearing Walls } & Low-Rise & $1-2$ & 1 & 15 \\
\hline 35 & URMM & & Mid-Rise & $3+$ & 3 & 35 \\
\hline 36 & $\mathrm{MH}$ & Mobile Homes & & All & 1 & 10 \\
\hline
\end{tabular}




\section{Table 3H(f). Occupancy Classes-HAZUS Codes}

\begin{tabular}{|l|l|}
\hline $\begin{array}{c}\text { HAzUS } \\
\text { clasuancy }\end{array}$ & \multicolumn{1}{c|}{ Description } \\
\hline AGR1 & Agriculture Facilities and Offices \\
\hline COM1 & Retail Trade \\
\hline COM2 & Wholesale Trade \\
\hline COM3 & Personal and Repair Services \\
\hline COM4 & Professional/Technical Services \\
\hline COM5 & Banks \\
\hline COM6 & Hospital \\
\hline COM7 & Medical Office and Clinic \\
\hline COM8 & Entertainment \& Recreation \\
\hline COM9 & Theaters \\
\hline COM10 & Parking Garages \\
\hline EDU1 & Grade Schools and Admin O Offices \\
\hline EDU2 & Colleges and Universities \\
\hline GOV1 & Government - General Services \\
\hline GOV2 & Government - Emergency Response \\
\hline IND1 & Heavy Industrial \\
\hline IND2 & Light Industrial \\
\hline IND3 & Food/Drugs/Chemicals \\
\hline IND4 & Metals/Minerals Processing \\
\hline IND5 & High Technology \\
\hline IND6 & Construction Facilities and Offices \\
\hline REL1 & Churches and Non-Profit Organizations \\
\hline
\end{tabular}




\begin{tabular}{|l|l|}
\hline RES1 & Single Family Dwellings \\
\hline RES2 & Manufactured Housing \\
\hline RES3A & Duplex \\
\hline RES3B & 3 to 4 Units \\
\hline RES3C & 5 to 9 Units \\
\hline RES3D & 10 to 19 Units \\
\hline RES3E & 20 to 49 Units \\
\hline RES3F & At lease 50 Units \\
\hline RES4 & Temporary Lodging \\
\hline RES5 & Institutional Dormitories \\
\hline RES6 & Nursing Homes \\
\hline UNK & Unknown \\
\hline
\end{tabular}

NIBS (2002) and California Governor's Office of Emergency Services (2004).

\section{Table 3I. Event (Earthquake) Information}

CAUTION: There are two forms of Subscripting for most Event tags, each with a distinct, specific use and purpose:

Subscripting certainly may be used to show Event.... solutions from multiple institutions (which we recommend), or to show a history of solutions from one institution (which we do not recommended). The form of that Subscripting is "Event.Hypocenter(n)....".

Similarly, a Subscript may be used when a record contains the traces of more than one Event, as may happen in very active aftershock and swarm sequences. This form of that Subscripting, in contrast, is "Event(m)....". This is a form of Subscripting that should never be used for any other purpose or confusion may result about what information goes with what event on a multiple-Event record. 
If any of the Event.Hypocenter.... tags are given, then all marked CONDITIONAL are REQUIRED:

Event $[(\mathrm{m})]$. CommonName_txt $=$ "<value $>$ ";

I| A common name for this earthquake, such as "1994 Northridge".

Event [ (m) ].Hypocenter[ ( $\mathrm{n})]$. Agency_txt = "<value>";

Originating provider (Agency from table 3E). This Agency

applies to the next 11 tags. If an Event.Hypocenter is

provided, this tag is REQUIRED.

Event [ (m) ]. Hypocenter [ ( n ) ]. Identifier_txt $=$ "<Agency's Identifier $>$ ";

(Unique) identifying string issued by the Agency

issuing the Event information. for example, "nc51153328",

or "[Test] Record of ..."

Event $[(\mathrm{m})] \cdot$ Hypocenter $[(\mathrm{n})] \cdot$ DateTime_txt $=$

$$
\text { "YYYY-MM-DD hh:mm:ss.s[ss][Z|[+|-]hh[:mm]]"; || Recommended }
$$

DateTime is either the date of computation or the date

these data were obtained from the Agency. It is not the

origin time of the Event, which is the next tag:

Event [(m) ].Hypocenter[ (n) ].OriginTime.DateTime txt =

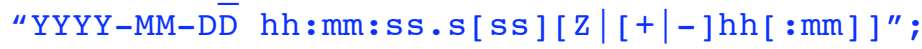

| | CONDITIONAL

ISO DateTime of Event origin time. Do not use

Event.Hypocenter.DateTime for origin times.

Event [(m)].Hypocenter[(n)].OriginTime.TimeError_dbl = <value> s;

|| Estimated two-sigma error

Event [ (m) ].Hypocenter[ ( $\mathrm{n})]$. Latitude_dbl = <value> deg;

|| Positive North, decimal degrees

Event [ (m) ].Hypocenter[ ( $\mathrm{n})]$. Longitude dbl = <value> deg;

|| Positive East, decimā degrees

Event [ (m) ].Hypocenter [(n)].Depth_dbl = <value><units>;

|| Depth below datum (km preferred)

Event [ (m) ].Hypocenter[ (n) ].HorizontalDatum_txt = "<from table 3G>";

|| "Horizontal" or "Mixed" datüm (rarely known)

Event $[(\mathrm{m})]$. Hypocenter $[(\mathrm{n})]$.VerticalDatum_txt $=$ "<value table $3 \mathrm{G}>$ ";

|| "Vertical" or "Mixed" datum (rarely known)

| Optional

|| CONDITIONAL

|| CONDITIONAL

| | CONDITIONAL

| Optional

|| Optional 
Event [ (m) ].Hypocenter[ (n)].HorizontalError_dbl = <value $><$ units $>$; Event $[(\mathrm{m})]$. Hypocenter[ $(\mathrm{n})]$.VerticalError_dbl $=\langle$ value $\rangle<$ units $>$;

( $\mathbf{k m}$ is the preferred for <units>)

We note the possibility of representing a full uncertainty

ellipsoid and seek comment on this matter. For now, these

tags describe only an error spheroid.

Event [ (m) ].Hypocenter[ (n)].URL_txt $=$ "<Web address $>$ ";

Event [ (m) ].Hypocenter [ ( $\mathrm{n})]$. Citation_txt $=$ "<Citation $>$ ";

Event [ (m) ].Hypocenter [ ( $\mathrm{n})]$. Comments txt $=$ "<Comments $>$ ";

Any Comments or Web site URL regarding these Event data.

If in Comments you need more than one line of text for a

particular $(\boldsymbol{n})$, put them all into one, long string with

"lines" separated by the character string "||n|".

Magnitudes, moment tensors, focal solutions, and ShakeMap tags may or may not be from the same Agency as the rest of the Event information, so those Agencies must be identified separately.

Event $[(\mathrm{m})] \cdot$ Magnitude $[(\mathrm{n})] \cdot$ Type_txt $=$

$$
\text { [ "Mw" | "MS"| "mb" | "MD" | "ML" | “Me" | “Mn" | .. ] ; }
$$

Event [ (m) ].Magnitude[(n)].Value_dbl = <value $>$;

Event [ (m) ].Magnitude [ (n)].ValueẼror dbl = "<value $>$ "

Event [ (m) ].Magnitude[(n)].Agency_txt $=$ "<from table $3 E(b)\rangle "$;

Event [ (m) ].Magnitude [ (n)].DateTime_txt = "YYYY-MM-DD [ ...]";

Event $[(\mathrm{m})]$. Magnitude [ ( $\mathrm{n})]$. NumberofStationsused int $=\langle$ value $\rangle$;

Event $[(\mathrm{m})]$.Magnitude[( $\mathrm{n})]$. URL_txt $=$ "<Web address $>$ ";

Event [ (m) ].Magnitude[ (n) ]. Citation_txt = "<Citation $>$ ";

Event $[(\mathrm{m})]$.Magnitude $[(\mathrm{n})]$.Comments txt $=$ "<Comments $>$ "

The CONDITIONAL Eveñt.Magnitude tags above are all REQUIRED

if any of them is given.

ValueError is optional and is the accuracy of Value. (The DateTime is either the date ( \pm time) when the Agency computed

this magnitude, or the date ( \pm time) when these data were

( obtained from the named Agency. Omit if unavailable.) 
The strings "Mw", "MS", "mb", "MD", "ML", "Me", and "Mn"

mean, respectively: the Moment Magnitude (Hanks and Kanamori, 1979).

the teleseismic 20-s surface-wave Magnitude (Gutenberg and

Richter, 1956); the teleseismic body-wave Magnitude (IASPEI,

2005, formula); the local-Event coda-duration Magnitude; any

equivalent of the Wood-Anderson local-Event Richter Magnitude

(Richter, 1935); the energy Magnitude (Choy and Boatwright,

1995); and the Nuttli Magnitude (Nuttli, 1963).

This is a USER-EXTENSIBLE LIST. Any user-defined magnitude name must begin with "User's own: " and end with the short

name of the user's magnitude method. For example, one could

name a magnitude "Mx" and thus indicate "User's own: Mx".

Event.Magnitude.NumerofStationsused is the number of stations contributing to the computation of this Magnitude.

URL is Recommended for alternately defined magnitudes, "Other", and for any "Mw" defined in a manner significantly different from that used by the USGS. The USGS equation is an IASPEIrecommended modification of the method of Hanks and Kanamori (1979; equation (7)) (which rearrangement makes a difference of only about 0.03 units):

$$
M w=(2 / 3)(\log (M o)-16.1) \text {, }
$$

where Mo is in units of dyne-cm. Since there is such a small difference from the original Hanks and Kanamori equation (7), that equation also may be used for "Mw" without giving a URL:

$$
M w=(2 / 3) \log (M 0)-10.7 \text {. }
$$

In any case, this URL should point to a complete definition of the magnitude indicated as "Other" or "Mw", including the computational method used. 
If, in Comments, you need more than one line of text

for a particular Event(m), or a particular solution for

Magnitude(n), put it all into one string with "lines"

separated by the character string "/nn".

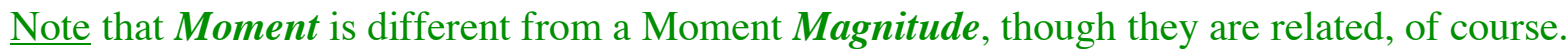

Event $[(\mathrm{m})]$.Moment.Value_dbl $=<$ value $><\mathrm{Nm} \mid$ dyne-cm $>$;

Event $[(\mathrm{m})]$.Moment.Agency txt $=$ "<table $3 \mathrm{E}>$ ";

Event $[(\mathrm{m})]$.Moment.DateTime_txt = "YYYY-MM-DD [ hh:mm:ss[z|[+|-]hh[:mm]]";

Event $[(\mathrm{m})]$.Moment.URL_txt $=$ "<Web address $>$ ";

Event $[(\mathrm{m})]$.Moment.Citation txt $=$ "<Citation $>$ ";

Event $[(\mathrm{m})]$. Moment. Comments_txt $=$ "<Comments $>$ ";

DateTime is either the date ( \pm time) of computation or
when these data were obtained from the named Agency.
In some cases, the Moment will need an explanation,
Comments. URL points to some reference.

Event [ (m) ].FaultRupture [ ( $\mathrm{n})]$. Length dbl $=\langle$ value $\rangle<$ units $>$;

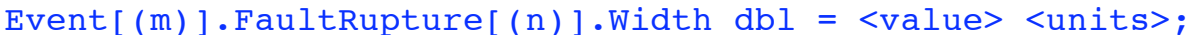

Event $[(\mathrm{m})]$. FaultRupture [ (n)]. SurfaceArea_dbl $=\langle$ value $><$ units $>$;

Event [ (m)].FaultRupture $[(\mathrm{n})]$. MeanSlip db $\bar{l}=\langle$ value $><$ units $>$;

Event $[(\mathrm{m})]$.FaultRupture $[(\mathrm{n})]$.BestSlip_dbl $=\langle$ value $><$ units $>$;

Event $[(m)]$. FaultRupture $[(n)] \cdot$ RiseTime_dbl $=\langle$ value $\rangle$ s;

Event [ (m) ].FaultRupture [(n)].SlipVelocity dbl = <value > <units $\rangle$

Event $[(\mathrm{m})]$. FaultRupture $[(\mathrm{n})]$.StaticStress $\bar{D} r o p \_d b l=\langle$ value $\rangle<$ units $>$;

Event $[(\mathrm{m})]$.FaultRupture $[(\mathrm{n})] \cdot$ Mach_dbl = <value $\rangle\langle$ units $>$;

Event [ (m) ].FaultRupture [ (n)].ShallowestAsperityDepth dbl = <value><units>;

Event $[(m)]$.FaultRupture $[(n)] \cdot$ FaultName $[(i)] \_t x t="<\bar{n}$ ame $>$ ";

Event [ (m) ].FaultRupture[(n)].Agency_txt $=$ "<table 3E $>$ ";

Event $[(\mathrm{m})]$. FaultRupture $[(\mathrm{n})] \cdot$ DateTime_txt $=$

Optional

CONDITIONAL

CONDITIONAL

Optional

Optional

optional

Optional

Optional

Optional

Optional

Optional

Optional

Optional

optional

Optional

Optional

| CONDITIONAL

| | CONDITIONAL 
Event [ (m) ].FaultRupture [ (n)].URL_txt $=$ "<Web address $>$ ";

Event [ (m) ].FaultRupture [ (n)].Citation_txt = "<Citation $>$ "

Event $[(\mathrm{m})]$.FaultRupture $[(\mathrm{n})]$. Comments txt $=$ "<Comments $>$ ";

| Various measures or models of average rupture behavior for rupture model (n).

Meanslip might be estimated from Moment.value in units of dyne-cm and FaultRupture.SurfaceArea in units of $\mathrm{km}^{\wedge} 2$ as

$$
\text { Meanslip }=\text { Moment. Value } /\left(3.58 * 10^{11} * \text { SurfaceArea } * 10^{10}\right) \text {. }
$$

Similarly, staticstressDrop in bars might be estimated from Moment.Value and SurfaceArea as

$$
\left.(7 / 16) * \text { Moment.Value / (SurfaceArea * } 10^{10} / \pi\right)^{1.5} / 10^{6} \text {. }
$$

Mach is the ratio of rupture velocity to $S$-wave velocity.

For example, FaultName might be "San Andreas fault". It is the fault on which the rupture is believed to have occurred. The (i) is for situations like the 1992 Landers earthquake, where the rupture subsumes faults with several names.

If an Event.MomentTensor is specified, all the CONDITIONAL tags below are REQUIRED:

Event $[(\mathrm{m})]$. MomentTensor[ (n)].Agency_txt $=$ "<table 3E $>$ ";

Event $[(\mathrm{m})]$. MomentTensor $[(\mathrm{n})]$. DateTime txt $=$

"Y̌YYY-MM-DD [ hh:mm:ss[z|[+|-]hh[:mm] ] ]";

DateTime is either the date ( \pm time) of computation or

| | CONDITIONAL

when these data were obtained from the named Agency. 
Event $[(\mathrm{m})] \cdot$ MomentTensor $[(\mathrm{n})] \cdot$ CoordinateSystem_txt $=$

$$
<"[\bar{U} \mid \mathrm{D}][\mathrm{N} \mid \mathrm{S}][\mathrm{E} \mid \mathrm{W}] " \text { in }(\mathrm{x}, \mathrm{Y}, \mathrm{z}) \text { order>; }
$$

These are the directions of the positive axes. The most common

coordinate system is that of $A k i$ and Richards (1980), that is,

Coordinatesystem "NED".

However, Harvard moment tensors are Coordinatesystem "USE" .

Event [(m)].MomentTensor[(n)].M(i)_cpx = <value $\quad[\mathrm{Nm} \mid$ dyne-cm ];

| These are the six values of a symmetric moment tensor in

Newton-meters or dyne-centimeters $(n=1,2,3,4,5,6)$. They

are given as a vector in this order only:

$$
\left[\begin{array}{llll}
1 & 2 & 3 \\
& 4 & 5 \\
& & 6
\end{array} \mid\right.
$$

Again, while the above is the most common in numerical processing, Harvard does it differently:

165

24

31
| | CONDITIONAL

| | CONDITIONAL 
Event [ (m) ].MomentTensor[ (n)].URL_txt $=$ "<Web address $>$ ";

Event [ (m) ].MomentTensor [ (n)].Citation_txt $=$ "<Citation $>$ " ;

Event $[(\mathrm{m})]$.MomentTensor $[(\mathrm{n})]$.Comments txt $=$ "<Comments $>$ ".

| If you need more than one line of text in comments,

use one long string with the "lines" separated by

the character string "/n|".

Event $[(\mathrm{m})]$. FocalSolution $[(\mathrm{n})]$.Agency_txt $=$ "<table 3E $>$ "

Event $[(\mathrm{m})]$. FocalSolution $[(\mathrm{n})]$. DateTime txt $=$

"YYYY-MM-DD [ hh:mm:ss [Z|[+|-]hh[:mm]]]" .

Event [(m)].FocalSolution[(n)].Strike_dbl = <value> deg;

Event $[(\mathrm{m})]$. FocalSolution $[(\mathrm{n})] \cdot$ Dip_db $\bar{l}=\langle$ value $\rangle$ deg;

Event $[(\mathrm{m})]$.FocalSolution $[(\mathrm{n})]$.Rake_dbl $=\langle$ value $\rangle$ deg;

Event $[(\mathrm{m})]$.FocalSolution [(n)].Mechanism_txt $=$ "<Use NGA definitions $>$ ";

Event $[(\mathrm{m})]$. FocalSolution $[(\mathrm{n})]$.PaxisPlunge dbl $=\langle$ value $\rangle$ deg;

Event $[(\mathrm{m})]$. FocalSolution $[(\mathrm{n})]$. PaxisTrend_$\overline{\mathrm{d} b l}=\langle$ value $\rangle$ deg;

Event $[(\mathrm{m})]$. FocalSolution $[(\mathrm{n})]$. TaxisPlunge dbl $=\langle$ value $\rangle$ deg

Event [ (m) ].FocalSolution[(n)].TaxisTrend_dbl = <value> deg;

Event [ (m) ].FocalSolution [ (n)].URL txt $=$ "<Web address $>$ "

Event $[(\mathrm{m})]$. FocalSolution [ ( n ) ].Citation_txt $=$ "<Citation $>$ ";

Event $[(\mathrm{m})]$. FocalSolution $[(\mathrm{n})]$. Comments_txt $=$ "<Comments $>$ ";

All CONDITIONAL Focalsolution tags are REQUIRED if any of

them is given. As a group they are optional as are those

labeled Optional.

DateTime is either the date ( \pm time) of computation or when these data were obtained from the named Agency.

| | CONDITIONAL

CONDITIONAL

CONDITIONAL

CONDITIONAL

CONDITIONAL

\section{Optional \\ Optional \\ Optional \\ Optional \\ Optional}

Optional

Optional

Optional 
Strike is 0-360 degrees clockwise from North, Dip is 0-90 down to the right when facing in the strike direction (thus, the hanging wall is on the viewer's right), and Rake is rupture motion 0-360 degrees counterclockwise from the strike direction when viewed from the hanging wall side).

These are the same definitions used by $A k i$ and Richards $(1980)$, p.106.

Meanslip is the average amount of slip over the entire rupture surface, for example in $\mathrm{cm}$.

Mechanism is the NGA-style summary of the faulting mechanism:

\begin{tabular}{|c|c|}
\hline Value & Rake Angles \\
\hline \multirow{2}{*}{ "Strike Slip" } & $-180<$ Rake $<-150$ \\
\cline { 2 - 2 } & $-30<$ Rake $<30$ \\
\cline { 2 - 2 } & $150<$ Rake $<180$ \\
\hline "Normal" & $-120<$ Rake $<-60$ \\
\hline "Reverse" & $60<$ Rake $<120$ \\
\hline \multirow{2}{*}{ "Reverse-Oblique" } & $30<$ Rake $<60$ \\
\cline { 2 - 2 } & $120<$ Rake $<150$ \\
\hline \multirow{2}{*}{ "Normal-Oblique" } & $-150<$ Rake $<-120$ \\
\cline { 2 - 2 } & $-60<$ Rake $<-30$ \\
\hline
\end{tabular}

The four tags PaxisPlunge, PaxisTrend, TaxisPlunge, and Taxistrend may be used to describe inferred orientations of the maximum, " $P$ ", and minimum, " $T$ ", axes of the compressive principal stresses.

For Comments, If multiple "lines" of text are needed for a particular (n), join them into a single text string, with the line breaks indicated by the character string "/|n/". 
Event [ (m) ]. ShakeMap [ ( $\mathrm{n})]$. Agency_txt = "<table 3E>"; Event [(m)].ShakeMap [(n)].DateTime_txt = "YYYY-MM-DD [ hh:mm:ss [z|[+|-]hh[:mm]]"; Event $[(m)]$. ShakeMap $[(n)]$.URL txt $=$ "<Web address $>$;

Event [ (m) ].ShakeMap [ ( $)$ ]. Citation_txt = "<Citation $>$ ";

If one of these tags is given, the first three tags are

Recommended. As a group they are Optional.

DateTime is either the date ( \pm time) of computation or when

these data were obtained from the named Agency. URL is the

Web address of the ShakeMap for this Event. Agency is the

entity that computed the shakemap.

Event $[(\mathrm{m})]$.ShakeMap $[(\mathrm{n})]$.Comments_txt $=$ "<Comments $>$ ";

If multiple "lines" of text are needed for a particular

(n), join them into a single text string, with the line

breaks indicated by the character string "/nn/".

In the following, where one of the CONDITIONAL tags is present for a given rupture segment $(\boldsymbol{i})$, all other such CONDITIONAL tags are REQUIRED for that $(i)$ :

Event [(m)].DetailedFaultRupture[(n)].Segment[(i)].Latitude_dbl = <value> deg;

Event [ (m)].DetailedFaultRupture [(n)].Segment [(i)].Longitude dbl $=\langle$ value $\rangle$ deg;

Event [ (m) ]. DetailedFaultRupture [ (n)].Segment [ (i)].Depth_dbl $=\langle$ value $>\mathrm{km}$;

Event [ (m) ]. DetailedFaultRupture [ (n)]. Segment [ (i)]. Length_dbl $=\langle$ value $><$ units $>$;

Event [ (m) ]. DetailedFaultRupture [ (n)].Segment [(i)].Width dbl = <value > <units $>$ i

Event [ (m)].DetailedFaultRupture [(n)].Segment [(i)].Strikēdbl = <value> deg;

Event [ (m)]. DetailedFaultRupture [ (n)].Segment [ (i)].Dip_db $\bar{l}=\langle$ value $\rangle$ deg;

Event [ (m) ]. DetailedFaultRupture[(n)].Segment [ (i)].Rake dbl = <value> deg;

Event [(m) ]. DetailedFaultRupture[(n)].Segment[(i)].SurfaceArea_dbl = <value><units $>$;

These tags form vectors describing the geometry and the
sense of motion on each rupture-surface segment (i) in a

particular model (n) of a particular Event solution (m).

The Strike, Dip, and Rake are as defined (Aki and Richards, 1980 ) as in Event.Focalsolution. 
Latitude, Longitude, and Depth are of the "upper left" corner of the segment, as viewed from the hanging-wall side. Depth is relative to mean sea level.

SurfaceArea is the surface area of that segment(i) of the rupture model. Of course, this can be computed from Length and Width, so if both are given they must be consistent.

Event [ (m) ].DetailedFaultRupture [ (n)].Segment [(i)].MeanSlip_dbl = <value><units $>$; Event [ (m) ]. DetailedFaultRupture [ (n)].Segment [(i)].BestSlip_dbl $=\langle$ value $><$ units $>$; Event [ (m) ].DetailedFaultRupture [ (n)].Segment [(i)].RiseTime dbl = <value $>\mathrm{s}$; Event [ (m) ].DetailedFaultRupture [ (n)].Segment [ (i)].Slipvelocity_dbl=

<value> <units>; || Optional

Event[(m)].DetailedFaultRupture[(n)].Segment[(i)].StaticStressDrop dbl = $<$ value> <units>; || Optional

Event [ (m) ]. DetailedFaultRupture [ (n)].Segment [(i)].Mach dbl = <value><units>; Event [ (m)].DetailedFaultRupture [ (n)].Segment [ (i)].Shal <value><units>; || Recommended

Details of slip on Segment(i). Meanslip is the mean slip on that segment of the rupture surface, for example in meters. Mach is the ratio of rupture velocity to $S$-wave velocity.

ShallowestAsperityDepth (depth to the top of the shallowest asperity) is sometimes needed for ground-motion models.

Event $[(\mathrm{m})]$. DetailedFaultRupture[(n)].Agency_txt $=$ "<table 3E $>$ "; Event $[(\mathrm{m})]$. DetailedFaultRupture [ (n)].DateTime_txt =

The above is a detailed rupture model (n) supplied by

this Agency on this date ( \pm time), where Daterime is

either the model creation date or the date when it was provided for this VTF file):

Event [ (m)].DetailedFaultRupture [ ( n ) ].URL txt $=$ "<Web address $>$ ";

Event [ (m) ]. DetailedFaultRupture [ (n) ]. Citātion txt $=$ "<Citation $>$ " :

Event [ (m) ]. DetailedFaultRupture [ (n)].Comments_txt $=$ "<Comments $>$ ";

URL, Citation, and Comments, can be used to point to or

provide a more detailed description and/or attribution

of the DetailedFaultRupture(n) model. 
To be clear: ( $\boldsymbol{m}$ ) is a particular Event, the (n) above

indicates a particular model while (i) above indicates

a particular rupture segment within that model. (There

will often be an (i), sometimes an (n), but only rarely

an (m) which would indicate one of several Events

recorded in this single Dataseries.)

\section{Table 3J(a). Data-Acquisition Unit (DAU; Recorder; Data Logger) Information}

"DAU" means "Data Acquisition Unit", the recording, timing, and telemetry system. It does not include the Sensor. (Tat is, a "DAS" is a $\boldsymbol{D A U}$ plus Sensors in ANSS parlance.)

Where not otherwise indicated, provide values for the particular recording stream issuing the time series leading to this DataSeries.

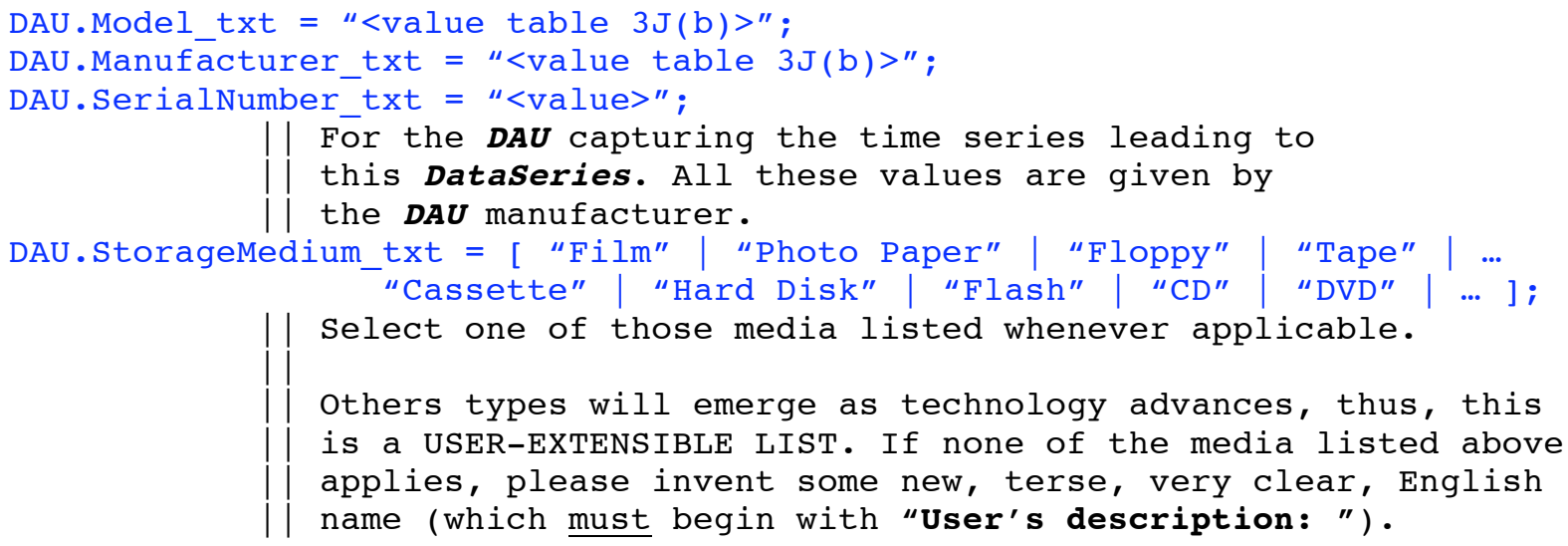


DAU.TotalChannels_int $=\langle$ value $>$;

Total number of channels in this DAU (cf.,

Array.TotalChannels, Array.TotalRecorders,

| Sensor.ArrayChannel, and Sensor.DAUchannel).

DAU.TotalChannelsRecorded int $=\langle$ value $>$;

|| Total number of channels in this DAU.

DAU.ChannelGain $d b l=\langle$ value $\rangle d B$

|| The total gain applied between the output of the sensor and

the input of the ADC (as decibels, so that zero means "no

gain applied", "unity gain").

DAU.AntiAliasFilter [ (m) ].Corner_dbl = <value $\rangle \mathrm{Hz}$;

|| Analog or digital anti-alias (low pass) filter $-3 \mathrm{~dB}$ point.

DAU.AntiAliasfilter[(m)].Decay dbl = <value> dB/octave;

DAU.AntiAliasfilter $[(\mathrm{m})]$. Comments_txt $=$ "<value $>$ ";

Generally, the analog anti-alias filter roll-off corner and

decay rate, but sometimes also a digital downsampling filter

(usually part of a "delta-sigma" ADC but sometimes computed

in a signal-processing unit to create multiple "streams" of

recorded data taken at differing sample rates).

The two CONDITIONAL tags are REQUIRED unless (as we advise)

the anti-alias filter(s) are subsumed as a parts of the

DAU.Transferfunction. If they can be subsumed in this way,

then these tags are Avoid.

Both the analog anti-alias filter and any digital "anti-

alias" downsampling filter(s) properly are part of and

should be described as part of (often "most of") the

DAU.TransferFunction rather than being separated out and

called an AntiAliasfilter. These AntiAliasfilter tags

are provided as alternative, lesser descriptions for

use only when information is scarce. 


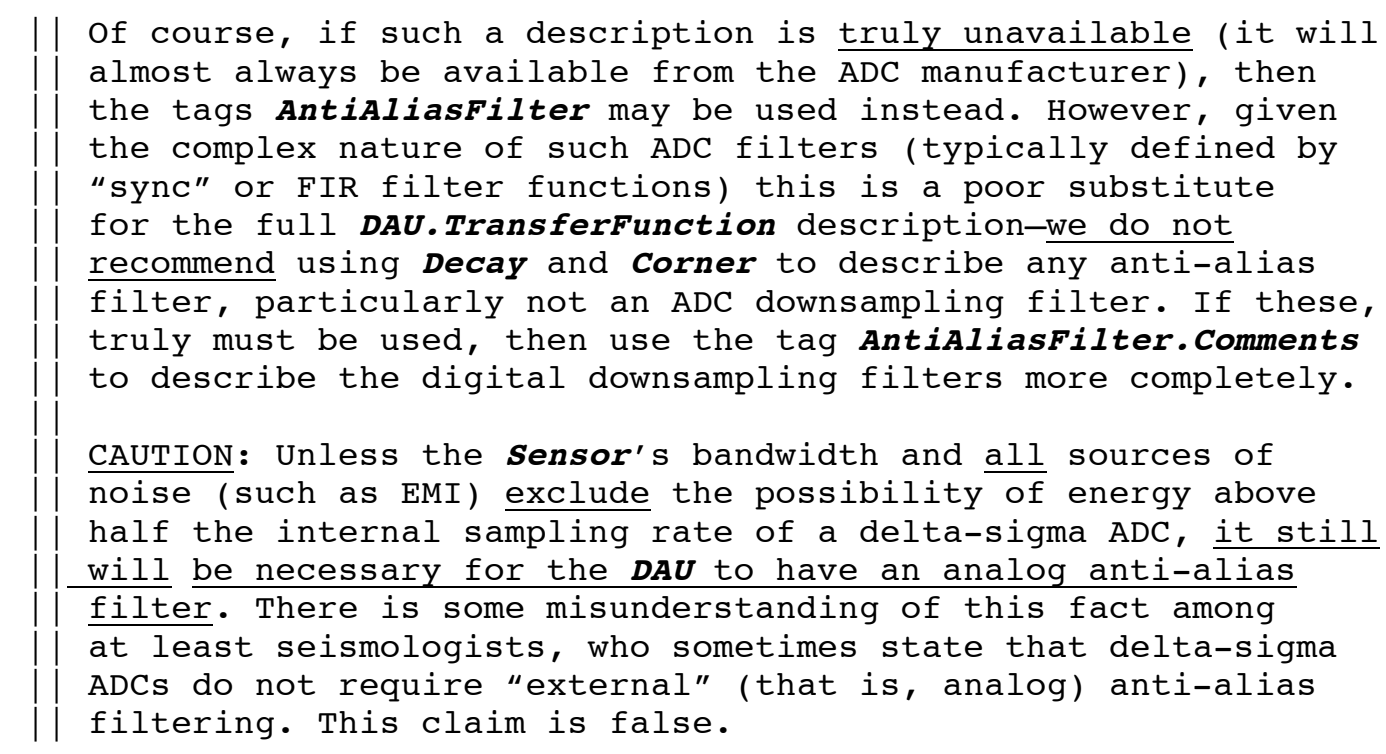

The transfer function of the $\boldsymbol{D A} \boldsymbol{U}$ is best described by poles and zeroes, or by their polynomial equivalents, ideally in the Laplace $(S)$ plane but alternately in the $z$-plane. These are the preferred forms.

If little is known about the TransferFunction, you may use the DAU.AntiAliasFilter.Corner, Comments, and DAU.ChannelGain tags for at least a general description of the DAU. Remember to include Comments when the ADC is a delta-sigma type, that is, contains a digital downsampling filter, since those alternative descriptions typically will not describe such filters accurately. 
DAU.TransferFunction.FilterName and one or the other set of tags below are jointly REQUIRED to the degree of a sufficient description whenever any is given. The Constants may or may not be needed, They default to a real value of one (1) if not provided (and the imaginary part will default to $0 j$ of omitted, leaving a real).

Detailed definitions of these Filter and TransferFunction tags is to be found in the comments describing Processing.Filters.

DAU.TransferFunction.FilterName txt $=$ "<from table $30(\mathrm{a})>$ ";

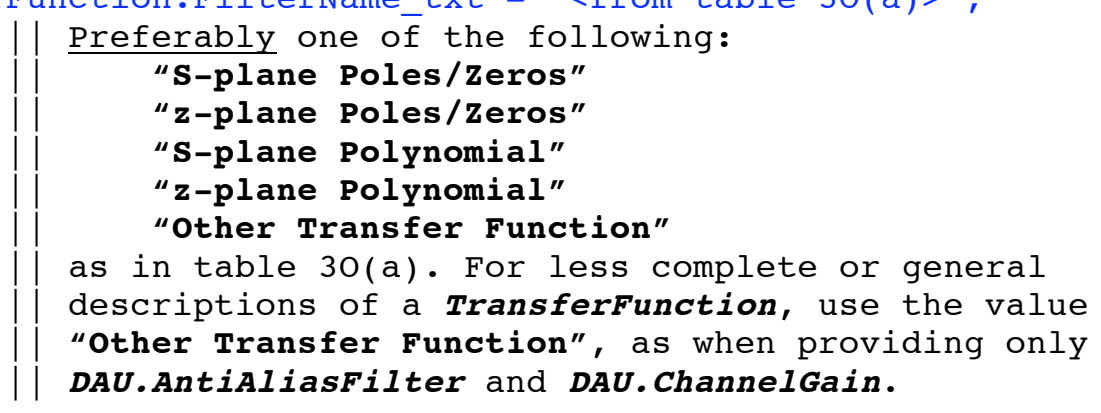

|| CONDITIONAL

DAU.TransferFunction.Causality txt = [ "Causal"| "Acausal" | "Zero Phase" ];

The causality of the DAU.TransferFunction is implicit in
either of the following formal descriptions, but may be
indicated here if desired. Modern delta-sigma recorders
are in all cases lagged "Zero Phase", though this fact
matters primarily at high frequencies.

| Optional

Either the set:

DAU.TransferFunction.Polezero.Numerator.NumberofRoots int $=\langle 0,1,2, \ldots>$; DAU.TransferFunction.Polezero.Denominator.NumberOfRoots_int $=<0,1,2, \ldots>$; DAU.TransferFunction.Polezero.Numerator.Root $(\mathrm{m}) \mathrm{cpx}=\langle$ real> $\langle$ imaginary $\rangle$;

DAU.TransferFunction.PoleZero.Denominator.Root $(\bar{m})$ _cpx $=\langle$ real> <imaginary>;

DAU.TransferFunction.Polezero.Numerator.Constant_c px $=\langle$ real $\rangle$ <imaginary $\rangle$;

DAU.TransferFunction.Polezero.Denominator.Constant_cpx $=\langle$ real $><$ imaginary>;

CONDITIONAL

CONDITIONAL

CONDITIONAL

CONDITIONAL

CONDITIONAL

CONDITIONAL 
or the set:

DAU.TransferFunction.Polynomial. Numerator.NumberOfCoeff int $=\langle 1,2,3, \ldots\rangle$;

DAU.Transferfunction.Polynomial.Denominator.NumberofCoeff int $=\langle 1,2,3, \ldots\rangle$;

DAU.TransferFunction.Polynomial.Numerator.Coefficient (m)_cpx $=\langle$ real> <imaginary $>$

DAU.TransferFunction.Polynomial.Denominator.Coefficient (m)_cpx =

$$
\text { <real> <imaginary>; }
$$

DAU.CountSize dbl $=\langle$ value $>\langle$ units $>$;

Size of one count (that is, the least significant bit) of the

$D A U$ analog-to-digital converter. <units> may be $\mathrm{V}, \mathrm{mV}, \mathrm{uV}$,

g_local, mg_local, ug_local, g_standard, mg_standard,

ug_standard, $\mathrm{cm} / \mathrm{s} / \mathrm{s}$, or any other time-series unit that

makes sense in this context.

DAU.Fullscale dbl = <value $>\langle$ units $>$;

\section{Same units as DAU.Countsize but the (two-sided}

full-scale range of the digitizer. For example, a

Quanterra $Q 330$ has $40 \mathrm{~V}$ peak-to-peak differential

inputs, so <value> would be 40 .

DAU.WordLength int $=\langle$ value $>$;

| In bits. For example, and ADC (analog-to-digital converter) recording in two's-compliment integers of length $\mathrm{N}$, the ADC output values will be in the range $\pm\left(2^{\mathrm{N}-1}-1\right)$. So for $\mathrm{N}=16$, the range is \pm 32767 (a few systems also allow -32768 , a

|| slight asymmetry inherent in two's-complement encoding).

DAU.DynamicRange_dbl $=\langle$ value $\rangle$ dB;

Effective system dynamic range, sensors plus recorder, as defined by ANSS, near $1 \mathrm{~Hz}$ or mid-band: Ratio between justclipping sine wave RMS and noise RMS in one-half-octave bin centered at (about) $1.0 \mathrm{~Hz}$ (if that is well inside the passband), or otherwise near the center of the pass-band. 
DAU.EffectiveBits_dbl = <value>;

| Bits $=\log _{2}(<$ total ADC counts $>/(2 *<$ RMS noise counts $>))$.

Equally, the peak-to-peak g's versus the RMS noise g's are

reduced to "bits" by a base-2 log. Therefore, let " $R$ " be

DAU.DynamicRange expressed as an amplitude ratio, hence

$\mathrm{R}=<$ peak-to-peak g's > / <root 2> / <noise RMS g's $>$. Thus,

DAU.EffectiveBits $=\log _{2}($ DAU.DynamicRange) -0.5 ; (It would

be "-1.0" to account for the double-sidedness of bit-counts

versus the single-sidedness of RMS, however, a dynamic range

involves a clipping sine wave while bit counts compares the

full range of the ADC-another square root of two separates

the metrics.)

DAU.CompressionUsed.Type_txt = [ "None" | "Steim" | "Reed-Solomon" | "Wavelet" | ... "Cosine" | "Other Lossless" | "Other Lossy" ]

DAU.CompressionUsed.Citation_txt = "<Citation>";

Any compression algorithm applied to the data prior to its

Optional

storage or transmission by the DAU. The Type should be

supplied. At least in cases of "Other ..." the Citation also

should be given.

DAU.Comments txt $=$ "<Comments $>$;

Any comments about the DAU or its operation (for

items not covered by defined DAU tags). If multiple

"lines" of text are needed, join them into a single

text string, with the line breaks indicated by the

character string "|ln|".

NOTE: Pre-Event and post-Event durations have been moved to Record data, since they may vary by record. Film digitizer Y-step has moved similarly. 


\section{Table 3J(b). DAU Models and Manufacturers}

\begin{tabular}{|c|c|}
\hline Model Name & Manufacturer \\
\hline \multicolumn{2}{|l|}{ Analog Recorders: } \\
\hline "USCGS Standard" & U.S. Coast and Geodetic Survey \\
\hline “AR-240" & \multirow{3}{*}{ Teledyne } \\
\hline “RFT-250” & \\
\hline “RFT-350" & \\
\hline “MO-2" & \multirow{2}{*}{ (New Zealand) } \\
\hline “MO2A" & \\
\hline “RMT-280" & Teledyne \\
\hline “SMA-1" & \multirow{4}{*}{ Kinemetrics } \\
\hline “SMA-2" & \\
\hline “SMA-3" & \\
\hline “CRA-1" & \\
\hline
\end{tabular}




\begin{tabular}{|c|c|}
\hline \multicolumn{2}{|c|}{ Digital Recorders: } \\
\hline “QDR" & \multirow{13}{*}{ Kinemetrics } \\
\hline "DSA-1" & \\
\hline "DSA-3" & \\
\hline “PDR-1" & \\
\hline "PDR-2" & \\
\hline "SSA-1" & \\
\hline "SSA-2" & \\
\hline "SSA-16" & \\
\hline “SSR-1" & \\
\hline "K2" & \\
\hline "Etna" & \\
\hline "Mt Whitney" & \\
\hline "Everest" & \\
\hline “DR-100" & \\
\hline "DR-200" & \\
\hline “DR-300" & Sprengnether \\
\hline “DR-3016" & \\
\hline \multicolumn{2}{|l|}{ "DR-3024" } \\
\hline "DCA-300" & \\
\hline "DCA-310" & Terraterh \\
\hline "DCA-333" & Terratecn \\
\hline \multicolumn{2}{|l|}{ "DCA-333R" } \\
\hline “GSR-12" & GeoSIG (Terratech a sometime \\
\hline "GSR-18" & reseller) \\
\hline "IDS-3602" & Terratech (IDS) \\
\hline "IDS-3602A" & Terratech (IDSA) \\
\hline
\end{tabular}




\begin{tabular}{|c|c|}
\hline "A700" & \multirow{4}{*}{ Geotech } \\
\hline "A800" & \\
\hline "A900" & \\
\hline "A900A" & \\
\hline "GEOS" & \multirow{4}{*}{ U.S. Geological Survey } \\
\hline “DST" & \\
\hline "Earthworm" & \\
\hline “TREMOR” & \\
\hline “Q4120” & \multirow{5}{*}{ Quanterra } \\
\hline “Q4128a" & \\
\hline “Q730" & \\
\hline "Q736" & \\
\hline “Q980" & \\
\hline “72A" & \multirow{3}{*}{ RefTek } \\
\hline “130-ANSS” & \\
\hline "130-SM" & \\
\hline "VSE-355G3" & Tokyo Sokushin \\
\hline \multicolumn{2}{|l|}{ Miscellaneous: } \\
\hline "Other Analog" & Unknown; legacy \\
\hline "Other Digital" & Unknown; legacy \\
\hline " & $\begin{array}{l}\text { USER-EXTENSIBLE LIST (use } \\
\text { established, terse, clear, } \\
\text { English names which must begin } \\
\text { with “User's description: ") }\end{array}$ \\
\hline
\end{tabular}




\begin{tabular}{|c|c|}
\hline Manufacturers of DAUs and of & sors : \\
\hline "Akashi" & \\
\hline "Canadian Geological Survey" & \\
\hline "GeoSIG" & \\
\hline "Geotech" & \\
\hline "Guralp" & \\
\hline "Kinemetrics" & \\
\hline "Lennartz" & \\
\hline "Mark Products" & \\
\hline "(New Zealand)" & \\
\hline "Quanterra" & \\
\hline "RefTek" & \\
\hline "Sprengnether" & \\
\hline "Streckeisen" & \\
\hline "Teledyne" & \\
\hline "Terratech" & \\
\hline "Terratech (IDS)" & \\
\hline “Terratech (IDSA)" & \\
\hline “Tokyo Sokushin" & \\
\hline “USCGS" & \\
\hline "U.S. Geological Survey" & \\
\hline "Wilcoxon" & \\
\hline “ & $\begin{array}{l}\text { USER-EXTENSIBLE LIST (use established, } \\
\text { terse, clear, English names, but not } \\
\text { necessarily the full, official corporate } \\
\text { name as long as it is quite clear. Any } \\
\text { user-defined manufacturer name must } \\
\text { begin with "User's description: ") }\end{array}$ \\
\hline
\end{tabular}




\section{Table 3K(a). Sensor Information}

Sensor.Model_txt $=$ "<value table $3 \underline{K}(\mathrm{~b})>" ;$

Sensor.Manufācturer_txt $=$ "<value table $3 \underline{J}(b)\rangle "$;

Sensor.SerialNumber_txt = "<value $>$ ";

For the sensor capturing the time series leading to

this Dataseries. All these values are given by the

Sensor manufacturer.

The orientation in space of a vector (the Sensor's positive active axis) is always uniquely and fully representable in the three-space on or near a planet's surface by two numbers, inclination and azimuth (the third element of this vector in effect represented by the GeoLocatio.Location and the figure of the planet).

\section{This two-element vector is the preferred description of orientation.}

Inclination is always given relative to plumb (Earth acceleration vector) from 0 to $180^{\circ}$ with zero meaning positive output for upward ground motion. (Absolute) Azimuth is always represented Eastward from North, ranging between 0 and 359.999..., with zero being North. Azimuth also may be represented relative to some stated Array (reference)

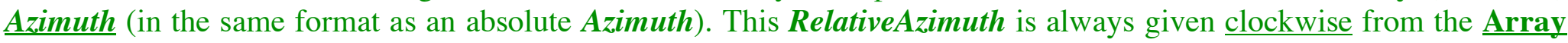
(reference) Azimuth, and is often the best option for an Array, such as in a structure. 
Absolute orientation:

Sensor.Azimuth.Value_dbl = <value> deg;

Sensor azimuth, clockwise from true geographic North (always

give zero "Azimuth" for an Inclination of 0 or $180^{\circ}$ ).

REQUIRED unless Sensor.RelativeAzimuth.Value and also

Array.Azimuth.Value are given.

Sensor.Inclination.Value_dbl = <value $>$ deg;

|| Positive up $=0$, horizontal $=90$, positive down $=180$

| | CONDITIONAL

Relative azimuth:

Sensor.RelativeAzimuth.Value dbl = <value $>$ deg;

| Like Sensor.Āzimuth.Value but 0 to 359.999... clockwise

from Array.Azimuth. Value rather than from true North.

In all cases, this compass direction must still be paired

with Sensor.Inclination.Value for a full description.

Array.Azimuth.Value dbl = <value> deg:

Azimuth of reference "North" versus true geographic North,

following the same rules as for Sensor.Azimuth.Value.

The absolute Azimuth of the Sensor is the (modulo 360) sum

of Sensor.RelativeAzimuth.Value and Array.Azimuth.Value

So this means, for example, that a Sensor oriented to cardinal

northeast but represented relative to

\section{Array.Azimuth.Value_dbl $=90$ deg}

(East) would be given as

Sensor.RelativeAzimuth.Value_dbl = 315 deg; 


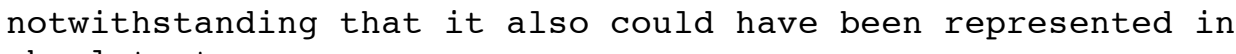

Sensor.Azimuth.Value_dbl = 45 deg;

Sensor.RelativeAzimuth.Value and Array.Azimuth.Value

are jointly REQUIRED if Sensor.Azimuth.Value is not given.

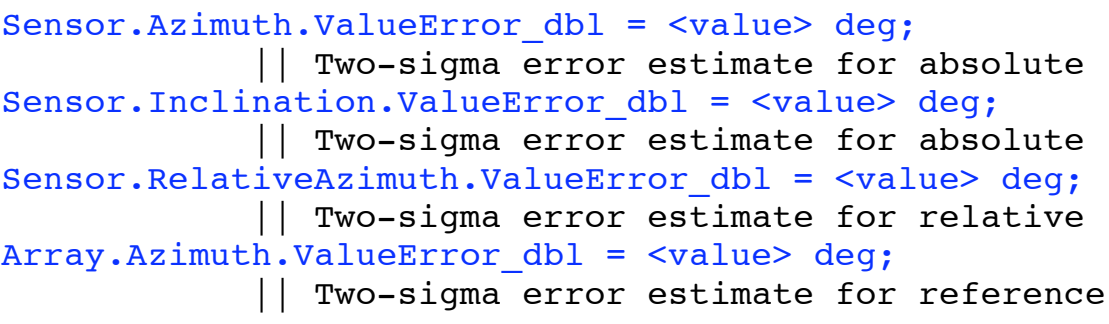

|| Two-sigma error estimate for reference

Alternate description:

Sensor.Orientation_txt $=$ "<from table $3 \mathrm{~K}(\mathrm{c})>$ ";

This tag is supplied only for backward compatibility

or, possibly, for preliminary use-its application

is discouraged.
|| Optional
|| Optional
|| Optional
|| Optional

Of the orientation tags above, Sensor.Azimuth.Value and Sensor.Inclination.Value are the preferred set.

However, in many Arrays the azimuths are given relative to a structure's orientation, or to some other reference azimuth, and in this case use the tag set Sensor.RelativeAzimuth.Value plus Array.Azimuth.Value, but by absolute Sensor.Inclination.Value since floors are more or less level.

Only as a last choice should you ever use Sensor.Orientation. (In any case, note that most of these can be translated into one of the other two representations.) 
Associated tags are:

Sensor.Arraychannel_int $=<$ value $>$;

|| Array-channel that this Sensor is on

Sensor.DAUchannel_int $=\langle$ value $>$;

The DAU-channel that this Sensor recorded on (if on

film, count down from the top, the first being 1 , not 0 )

(cf. Array.TotalChannels, Array.TotalRecorders,

and DAU.TotalChannels)

Sensor.Sensitivity dbl $=\langle$ value $>$ <units $>$;

Sensor.Sensitivity is in units, for film of cm/g_standard or $\mathrm{cm} / \mathrm{g}$ local, for example. <units> for other systems are

$\mathrm{V} / \mathrm{g}$ standard, V/g_local, $\mathrm{V} /(\mathrm{cm} / \mathrm{s} / \mathrm{s})$ or $\mathrm{V} /(<$ units $>)$, where

<units> is one of the other units of acceleration, velocity,

displacement, rotation, pressure, ustrain, and so forth.

Parentheses are required when <units> is compound,

as in the example.

NB: In all cases, this Sensitivity value is to be as measured as close as possible to the raw Sensor-thus, in the case of

FBAs and similarly "conditioned" Sensors, at the output of that

Sensor system. Sensitivity is to be measured before any DAU

amplification and filtering, so well before digitization.

Sensor.Fullscaleout_dbl = <value> <units>;

|| Peak output of the Sensor. The same measurement rules

as apply to Sensor.Sensitivity.

Sensor.FullscaleIn dbl = <value> <units $>$;

Peak unclipped Earth signal in <units> apropos to that

Sensor; generally g_local, g_standard, or $\mathrm{cm} / \mathrm{s} / \mathrm{s}$ for

accelerometers. The same measurement rules apply as

for Sensor.Sensitivity. 
Sensor.SensitivityContext_txt = "<where measured $>$ ";

| | Recommended

| The context in which sensor.Sensitivity,

sensor.Fullscaleout, and Sensor.FullscaleIn

were measured. For example, "At output of [ FBA |

geophone | sensor system ]".

Sensor.Causality_txt $=$ [ "Causal"| "Acausal" ];

$\mid \begin{aligned} & \text { All fully-analog Sensors are by definition "Causal". } \\ & \text { However, sensors containing digital signal conditioning, } \\ & \text { likely including those with a digital output, may } \\ & \text { contain a delta-sigma ADC or some other "Acausal" } \\ & \text { elements that make the whole "Acausal". } \\ & \text { The default is "Causal" since this is most often the case. } \\ & \text { This tag is REQUIRED if the Sensor (package) is in any } \\ & \text { way "Acausal" (most commonly for Sensor packages having } \\ & \text { built-in signal conditioning and digitizing and digital } \\ & \text { output). }\end{aligned}$

| | CONDITIONAL

The Sensor.TransferFunction is most completely and generally described by PoleZero or the equivalent Polynomial coefficients, preferably in the Laplace- $(S$-)plane, but alternately in the $z$-plane. These are the preferred forms. Alternate, less precise forms are given below those forms.

If TransferFunction.FilterName is given, then the specified set of tags are jointly REQUIRED, to the extent they form a complete representation of the TransferFunction.

In PoleZero representations, either the Numerator or the Denominator may have no roots in which case there will only be a Constant for that portion (and this may be omitted if it is equal to a real value of one (1) (the default value of Constant; the imaginary part will default to $0 j$ of omitted, leaving a real). The corresponding NumberOfRoots will be zero, no roots will be listed for that portion. It is common to gather both Constants into the Numerator, but that is not required.

Polynomial representations, of course, have a constant built in at the zeroth-order Coefficient, and M+1 Coefficients are always required (Subscripted from 1 through $\mathbf{M + 1}$ ), even if some of these are zeros. 
Detailed definitions of these filter classes is to be found among the comments describing Processing.Filters and in table 3O(a).

Sensor.TransferFunction.FilterName txt $=$ "<from table $30(a)>$;

|| CONDITIONAL

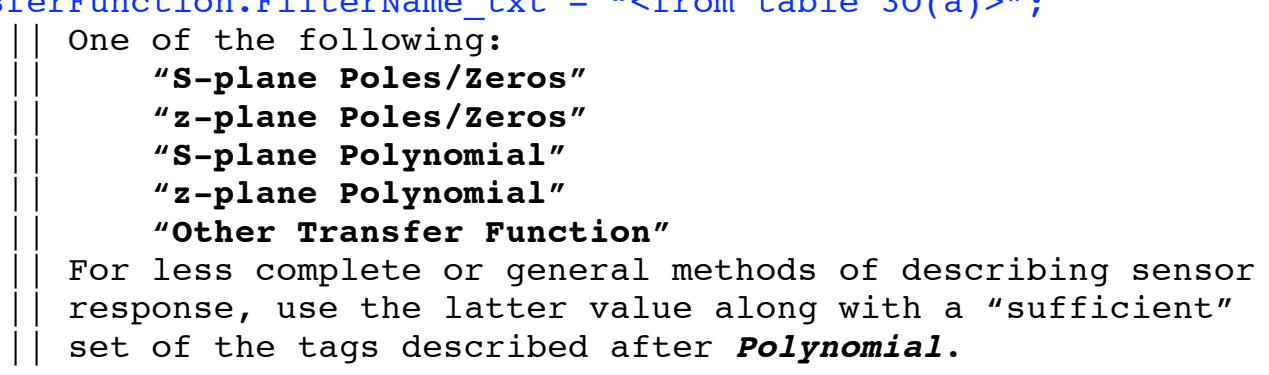

Use either the set:

Sensor.TransferFunction.Polezero.Numerator.NumberOfRoots_int $=<0,1,2, \ldots>$; Sensor.TransferFunction.Polezero.Denominator.NumberofRoots_int $=\langle 0,1,2, \ldots$. Sensor.TransferFunction.Polezero.Numerator.Root $(\mathrm{m}) \mathrm{cpx}=\langle\mathrm{real}\rangle\langle$ imaginary>; Sensor.TransferFunction.Polezero.Denominator.Root $(\bar{m})$ cpx $=<r e a l><$ imaginary>; Sensor.TransferFunction.Polezero.Numerator.Constant $\mathrm{cpx}=\langle$ real $\rangle$ <imaginary>; Sensor.TransferFunction.Polezero.Denominator.Constant_cpx $=\langle$ real $><$ imaginary $>$;

CONDITIONAL

CONDITIONAL

CONDITIONAL

CONDITIONAL

or the set:

Sensor.TransferFunction.Polynomial.Numerator.NumberofCoeff_int $=<1,2,3, \ldots .>$; Sensor.Transferfunction.Polynomial.Denominator.NumberOfCoeff_int $=<1,2,3, \ldots$; Sensor.TransferFunction.Polynomial.Numerator.Coefficient $(\mathrm{m}) \mathrm{cpx}=$ <real> <imaginary>; Sensor.TransferFunction.Polynomial.Denominator.Coefficient(m)_cpx $=$ 
Less complete or general descriptions of Sensor behavior:

Sensor.NaturalFrequency.Value_dbl = <value $\rangle \mathrm{Hz}$;

|| Sensor's naturāl frequency.

Sensor.Damping.Value_dbl = <value>;

Sensor's damping (as a fraction of critical).

This pair jointly REQUIRED if either is given.

Sensor.NaturalFrequency.ValueError_dbl $=\langle$ value $\rangle \mathrm{Hz}$;

Sensor.Damping.ValueError_dbl = <value $>$;

|| Estimated two-sigma errors.

Sensor.HighPass.Corner dbl = <value> Hz;

Sensor low-frequency corner $(0.0 \mathrm{~Hz}$ for many ( accelerometers)

Sensor.HighPass. Decay dbl = <value > dB/octave;

Sensor low-frequency roll-off, if

Sensor.HighPass.Corner is not zero.

This pair jointly REQUIRED if either is given.

Sensor.LowPass.Corner_dbl = <value> Hz;

Sensor high-frequency corner, if present in

addition to Sensor.NaturalFrequency.Value.

Sensor.LowPass.Decay_dbl = <value> dB/octave;

sensor high-frequency roll-off.

This pair jointly REQUIRED if either is given.

\section{| | CONDITIONAL \\ | | CONDITIONAL}

| Optional

| | CONDITIONAL

| | CONDITIONAL

| | CONDITIONAL

| | CONDITIONAL 


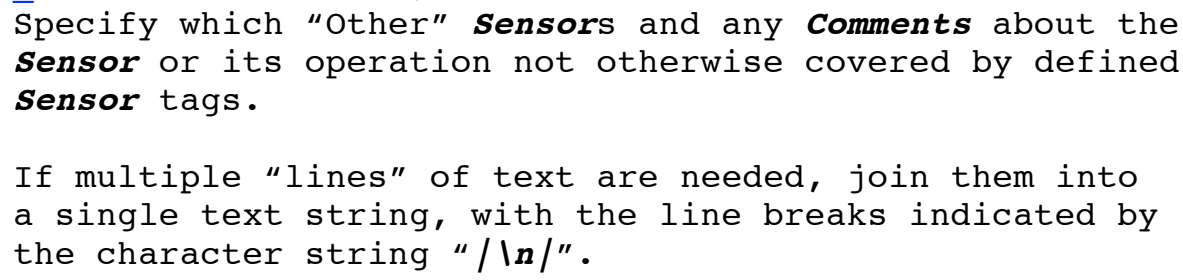

NOTES:

(1) We realize that some of the "required" tags will not always be known, particularly for legacy data-these tags still must be present in the VTF file and given the value NULL (no quotes).

(2) "Gain applied to sensor output ..." has moved to $\boldsymbol{D A U}$ information, because amplifiers and buffers are part of the recorder. In the case of high-output analog Sensors (for example, force-balance accelerometers), the internal circuitry is considered part of the Sensor.

(3) For Sensor azimuths, now see: Sensor.Azimuth.Value or Sensor.RelativeAzimuth.Value plus Array.Azimuth.Value or, as a last choice, Sensor.Orientation.

(4) For location offsets (in meters, for example) of GeoLocations in Arrays, now see GeoLocation.Location.NorthOffset, GeoLocation.Location.EastOffset, and GeoLocation.Loca-tion.ElevationOffset in table $3 \mathrm{~F}$. 


\section{Table 3K(b). Sensor Models (use Table 3J(b) for Manufacturers)}

\begin{tabular}{|c|c|}
\hline Model name & Manufacturer \\
\hline \multicolumn{2}{|l|}{ Accelerometers: } \\
\hline “Optical-Mechanical” (SMA, RFT,...) & Any \\
\hline “FBA-1" & \multirow{9}{*}{ Kinemetrics } \\
\hline "FBA-11" & \\
\hline “FBA-11" & \\
\hline “FBA-13" & \\
\hline "FBA-13DH" & \\
\hline "FBA-23" & \\
\hline “FBA-23DH" & \\
\hline "EpiSensor" & \\
\hline “EpiSensor ES-U” & \\
\hline "FBX-23" & \multirow{2}{*}{ Sprengnether } \\
\hline "FBX-26" & \\
\hline "SSA $120 "$ & \\
\hline "SSA 220" & Terratech \\
\hline \multicolumn{2}{|l|}{ “SSA 320" } \\
\hline “731A" & Wilcoxon \\
\hline “CMG-5” & Guralp \\
\hline \multicolumn{2}{|l|}{ Velocity Sensors/Seismometers: } \\
\hline "SS-1 Ranger" & Kinemetrics \\
\hline "S-3000" & Sprengnether \\
\hline
\end{tabular}




\begin{tabular}{|c|c|}
\hline “CMG-1” & \multirow{4}{*}{ Guralp } \\
\hline “CMG-3T" & \\
\hline “CMG-3ESP" & \\
\hline "CMG-40" & \\
\hline “STS-1" & \multirow{2}{*}{ Streckeisen } \\
\hline “STS-2" & \\
\hline “L4" & \multirow{2}{*}{ Mark Products } \\
\hline “L22D" & \\
\hline "SA-102A" & (New Zealand) \\
\hline \multicolumn{2}{|c|}{ Other Sensors (which may be specified in Sensor.Comments): } \\
\hline "Other Accelerometer" & \multirow{2}{*}{$\begin{array}{l}\text { Unknown or legacy sensor (in } \\
\text { preference, please use the } \\
\text { User-Extensible option and } \\
\text { provide a real model name) }\end{array}$} \\
\hline “Other Velocity seismometer" & \\
\hline "Pressure Sensor" & \multirow{5}{*}{$\begin{array}{l}\text { Various (in preference, } \\
\text { please use the User- } \\
\text { extensible option and provide } \\
\text { a real model name) }\end{array}$} \\
\hline "Dilatometer" & \\
\hline "Relative Displacement Sensor" & \\
\hline "Rotational Sensor" & \\
\hline "Other Sensor" & \\
\hline “ & $\begin{array}{l}\text { USER-EXTENSIBLE LIST (use } \\
\text { established, terse, clear, } \\
\text { English names which must } \\
\text { begin with "User's } \\
\text { description: ") }\end{array}$ \\
\hline
\end{tabular}

(It is clear that this table must grow. Please e-mail the authors or use the extensible-list option to communicate suggestions to us.) 


\section{Table 3K(c). Sensor Direction Codes for use with Sensor.Orientation only (Avoid)}

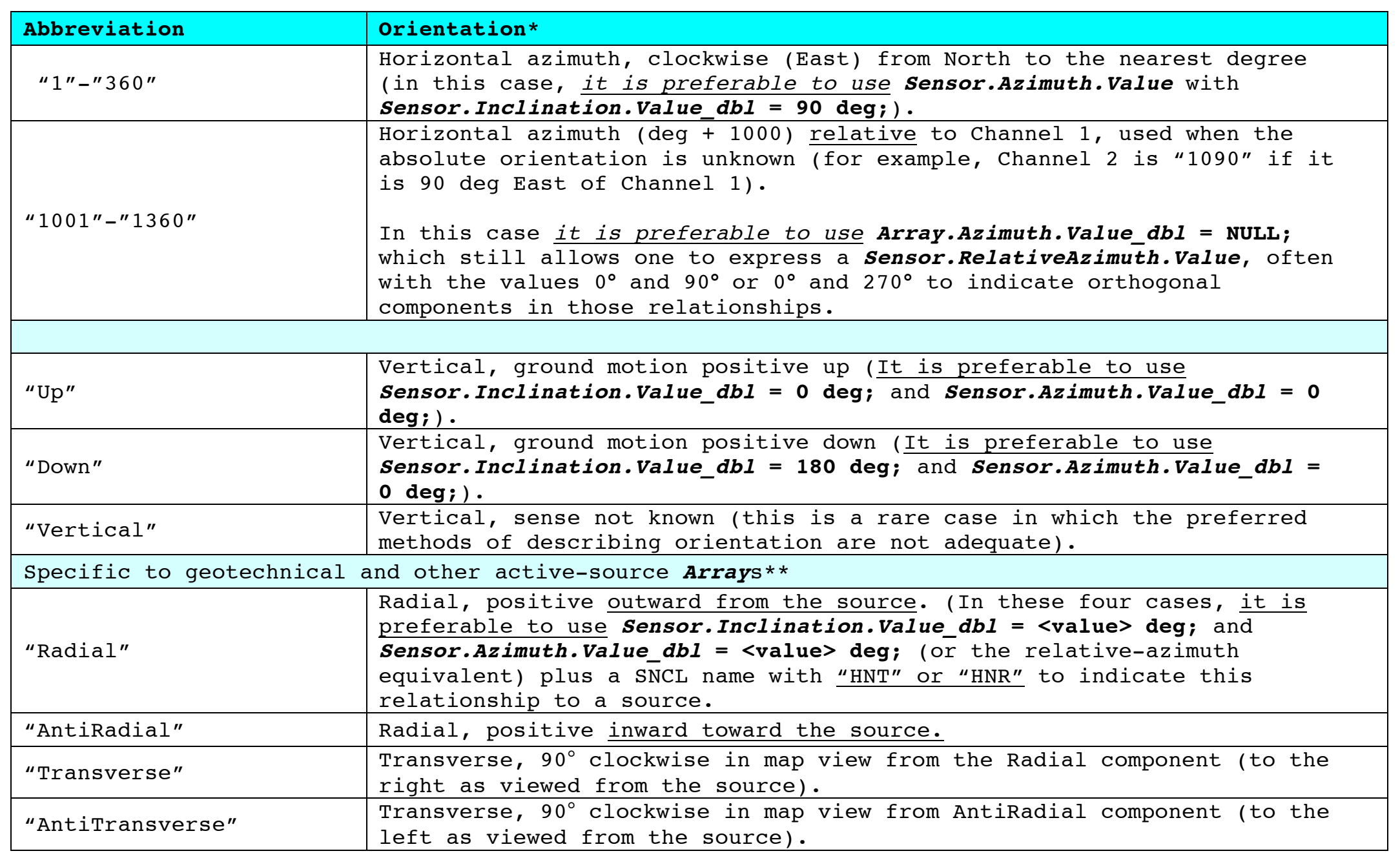




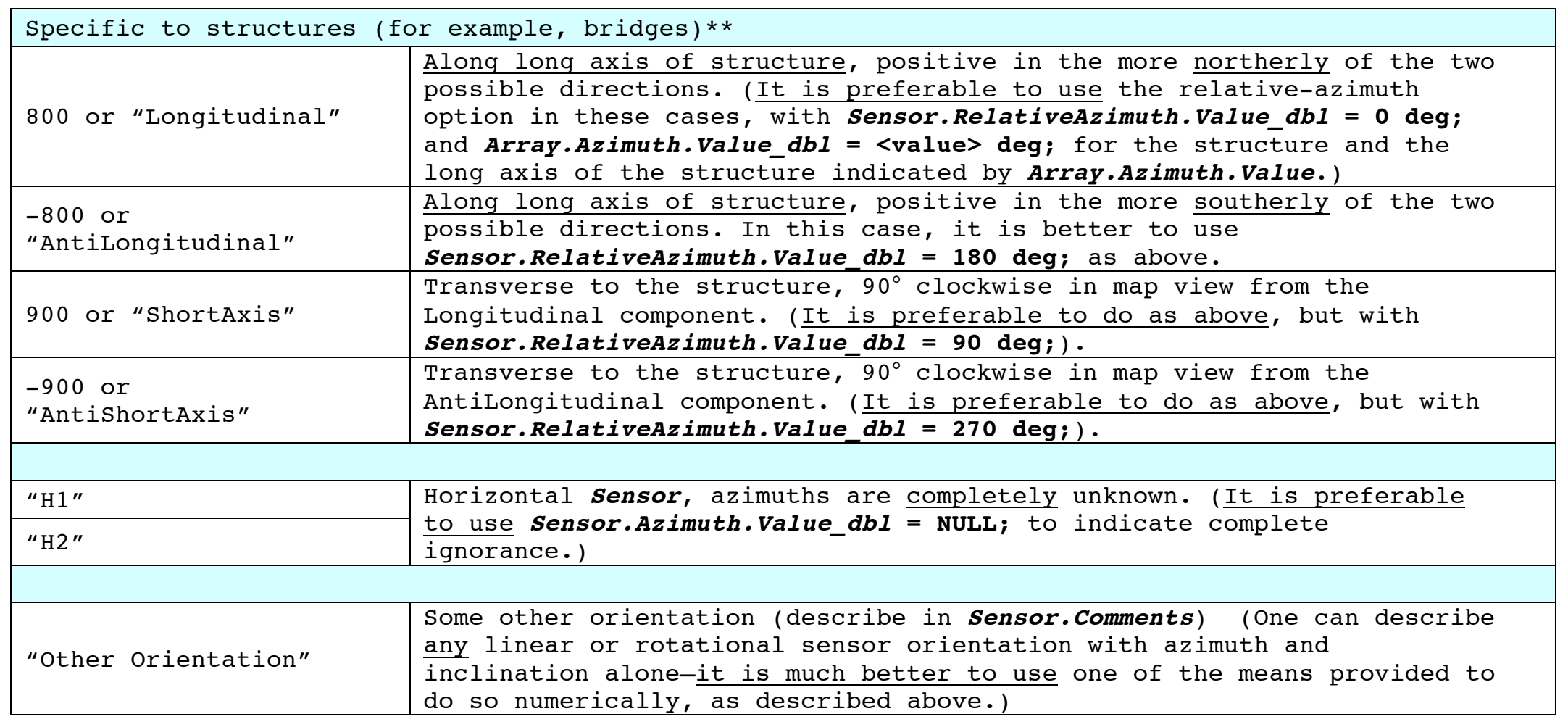

*The direction specified is the direction of motion of the ground (or the structural element to which the Sensor is attached) which corresponds to a positive value in the time series (or for film, of an upward motion of the trace).

**Definitions changed from v1.20 for clarity and to follow existing standards. 


\section{Table 3L. Data Series (Including Original): Time-Series or Spectrum Information}

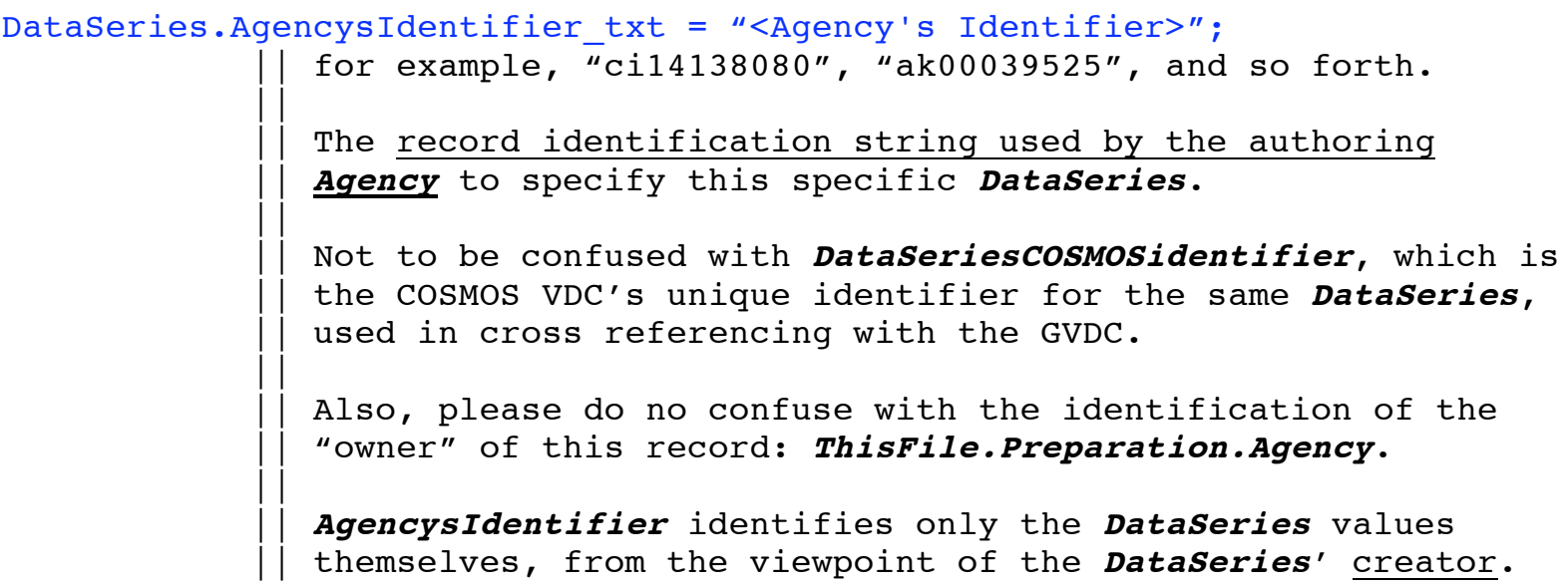

DataSeries.AgencysIdentifier_txt = "<Agency's Identifier>"; for example, "cil4138080", "ak00039525", and so forth.

Time-series time stamps (part of the abscissa definition for all time series):

RawSeries.FirstSampleTime.DateTime_txt $=$

"YYYY-MM-DD hh:mm:ss.sss[Z|[+|-]hh[:mm]"; $\quad \mid$ Optional | ISO DateTime (preferably to ms) of the first sample in the original file. 
DataSeries.FirstSampleTime.DateTime_txt =

"YYYY-MM-DD hh:mm:ss.sss [Z|[+|-]hh[:mm]]" ;

| | CONDITIONAL

ISO DateTime (preferably to $\mathrm{ms}$ ) of the first sample

in this file. REQUIRED for all time series,

whether or not the abscissa is implicit.

\section{Note that this is the primary time stamp for this}

file's time series (Dataseries) and is a key datum.

DataSeries.FirstSampleTime.JulianDay_int $=\langle$ value $>$;

The "Julian Day" (more correctly, the "Cardinal Day") is the

day of the year (from 1 to 366 ) and corresponds to "MM-DD" in

DataSeries.FirstSampleTime.DateTime. This tag is provided

for backward compatibility-the cardinal day is easily

computed and, therefore, redundant.

Note that there is no provision in our version of the ISO DateTime

for using a cardinal day, so "MM" and "DD" are required (but also

easily computed from the cardinal day).

Information related to time stamps:

RawSeries.FirstSampleTime.TimeError_dbl = <value $>$ s;

| Estimated or computed two-sigma error of

RawSeries.FirstSampleTime.DateTime.

RawSeries.FirstSampleTime.Source txt = "<from table 3M>".

|| REQUIRED if RawSeries.FirstSampleTime.DateTime

is present.

DataSeries.FirstSampleTime.CorrectionApplied_dbl = <value> s;

| Correction which has been applied to a recorded first-sample

time to arrive at DataSeries.FirstSampleTime.DateTime, the

start time of this time series. The sign of this correction

is in the sense:

DataSeries.FirstSampleTime.DateTime =

<first-sample time on recorder's chronometer>

+ DataSeries.FirstSampleTime.CorrectionApplied

Thus, a slow recorder clock produces a positive correction.

|| Recommended

|| CONDITIONAL

|| CONDITIONAL 
DataSeries.FirstSampleTime.TimeError_dbl $=\langle$ value $>$ s;

|| Estimated or computed two-sigma error of

Dataseries.FirstSampleTime.DateTime.

DataSeries.FirstSampleTime.Source_txt = "<from table 3M $>$ ";

|| Recommended

| REQUIRED

For spectral abscissa:

DataSeries.FirstBinfrequency dbl = <value $>\mathrm{Hz}$;

DataSeries.FirstBinPeriod_db $\bar{l}=\langle$ value $\rangle$ s;

| In the case of evenly sampled spectra, these tags are used in

to define an implicit abscissa for the Dataseries.

If DataSeries.PeriodBin is used as part of an implicit period abscissa, then Dataseries.FirstBinPeriod is required (because a 0-s period bin makes no sense). If DataSeries.FrequencyBin is used as part of an implicit frequency abscissa, then FirstBinfrequency may be used to define the lowest frequency bin. It defaults to $0 \mathrm{~Hz}$, which is apropos most power spectral densities and the DFT of a real time series. In all other cases, FirstBinfrequency must be given explicitly or the abscissa must be explicit.

In the case of an evenly-sampled DataSeries that does not have an explicit abscissa provided in the DataSeries listing matrix, exactly one of the following four is REQUIRED, along with DataSeries.FirstSampleTime.DateTime, DataSeries.FirstBinFrequency, or DataSeries.FirstBinPeriod, which together define an implicit abscissa. Note that the units used here must be the same as those declared by DataSeries.AbscissaUnits, one of the first two below for time series and one of the latter two for spectra. The chosen tag below must also make sense in combination with DataSeries.FirstSample-Time.DateTime, DataSeries.FirstBinFrequency, and DataSeries.FirstBinPeriod.

For implicit, evenly spaced time series, the time axis begins at zero seconds and proceeds to later time by intervals declared by DataSeries.SamplesPerSecond or DataSeries.SampleInterval. The absolute time of the first sample is given by DataSeries.FirstSampleTime.DateTime. In the case of evenly sampled spectra with implicit abscissa, the first bin 
always is assumed to be at $\mathbf{0 ~} \mathbf{H z}$ or $\mathbf{0 ~ s}$ (the latter does not appear to us to make sense so is unlikely to be used; the former makes sense for the DFT spectrum of a real-valued time series, which we anticipate will be the most common use of FrequencyBin).

DataSeries.SamplesPerSecond_dbl = <value $>\mathrm{Hz}$;

|| Sampling rate of time series

DataSeries.SampleInterval dbl = <value $>\mathrm{s}$ :

|| Sampling interval of time series

Dataseries.FrequencyBin_dbl = <value $>\mathrm{Hz}$;

DataSeries.PeriodBin dbl = <value > s;

|| Bin spacing in a spectrum. (For PeriodBin, the first bin

| at "zero period" might apply to PGA supplied with $\boldsymbol{s}_{a}$, for

example, but will more often make no sense. Its use will

be rare.)

RawSeries.SamplesPerSecond_dbl = <value> Hz;

|| Sampling rate of foregoing time series, or ...

RawSeries.SampleInterval_dbl = <value> s;

|| ... sampling interval of foregoing time series, or ...

RawSeries.UnevenSampling_txt = [ "Yes" | "No" ];

|... that the Dataseries was unevenly sampled, as for

hand-digitized optical records. Default is "No",

that is, even sampling.

RawSeries.AbscissaUnits_txt = "<units>";

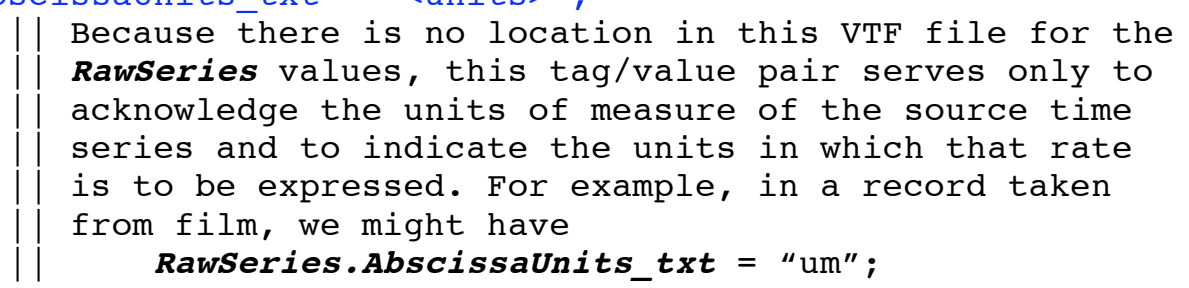

| | CONDITIONAL

|| CONDITIONAL

CONDITIONAL CONDITIONAL

|| Optional

| Optional

| Optional

| | CONDITIONAL 
DataSeries.AbscissaUnits is REQUIRED in all cases and is the single authoritative location in which the units of the abscissa are given (notwithstanding that those same units may well be used with related tags, and when so must at least not conflict with this RawSeries.AbscissaUnits). In the case of implicitly defined even sampling intervals, the abscissa values are implied by a combination of DataSeries.SamplesPerSecond, SampleInterval, FrequencyBin, or PeriodBin, plus a starting value, either DataSeries.FirstSampleTime.DateTime for a time series or DataSeries.FirstBin-Frequency or DataSeries.FirstBinPeriod for spectra.

In the case of uneven sampling of the DataSeries in this VTF file, or of an author's desire to provide an explicit abscissa for other reasons, AbscissaFormat also is REQUIRED and there must be an additional (first) column (or pair of columns if the abscissa is complex valued) in the main DataSeries listing matrix.

What one might have expected as the corresponding first entry in DataSeries.OrdinateFormat is not there, not prescribed by OrdinateFormat, but is instead given here as DataSeries.AbscissaFormat. Thus, the format of an entire row of the DataSeries listing matrix is a concatenation of AbscissaFormat with OrdinateFormat.

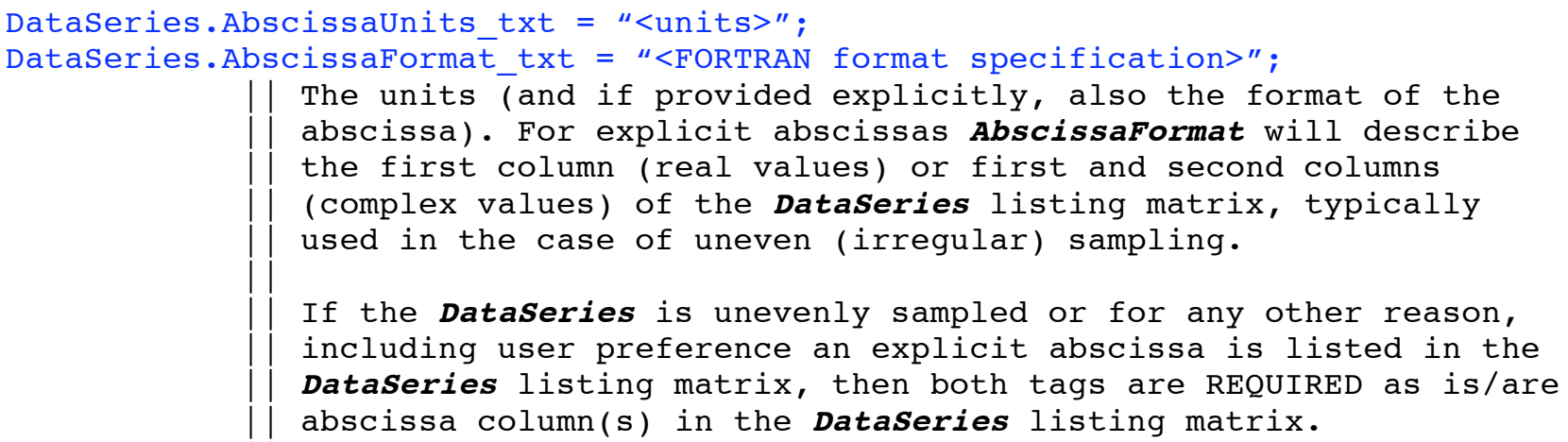


A single abscissa value is allowed for each row of the Dataseries listing matrix, however, this value can be either real or complex.

If it is complex, Abscissaformat must have two entries, generally

abutting and always given as a single text string containing one format specification for the real and one for the imaginary part

of each value. (Note that FORTRAN format-repetition counts are

not allowed, so there would have to be two explicit formats for

complex values.)

DataSeries.Span_dbl = <value $>$ [ $\mathrm{s} \mid \mathrm{Hz}$ ];

The time duration or bandwidth of the whole Dataseries.

Optional because it is computed easily from other values.

If given, therefore, it also must equal one of the following:

DataSeries.NumberofSamples / DataSeries.SamplesPerSecond

DataSeries.NumberofSamples * DataSeries.SampleInterval

DataSeries.NumberofSamples * DataSeries.FrequencyBin

DataSeries.NumberofSamples * DataSeries.PeriodBin

and so forth, OR, when there is/are abscissa column(s) in

the Dataseries listing matrix, the difference between the

last and first abscissa values.

RawSeries.Span_dbl = <value $>$ s;

Same thing, but for the original record (which

| Optional

| must have been a time series, of course). 
The following two tags relate to double-integration initial conditions. Also see RawSeries.Mean, DataSeries.Mean, Processing.InitialValue.Velocity and Processing.InitialValue.Displacement and/or the ultimate statement of baselinesproviding them explicitly with DataSeries.PhysicalParameter = "Acceleration Correction Baseline"; in a separate VTF file.

DataSeries.Pad.Firstoforiginal int $=\langle$ value $>$;

DataSeries.Pad.Lastoforiginal_int $=\langle$ value $>$;

CONDITIONAL

Both are REQUIRED if the DataSeries retains padding on either

CONDITIONAL

or both ends as used in Processing (as is recommended by Boore and Akkar, 2003) and also by us.

These are the (integer) indexes of first and last samples of

the unpadded part of the DataSeries (the first sample of the

padded trace contained in this VTF file being index 1).

For example, if 10 zeros were added to each end of a 100-point

original time series, then we would have

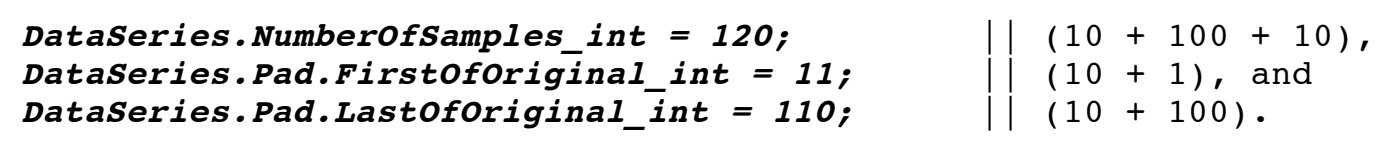

The same would apply if no padding was appended to the rear in this

example, except that the trace length would be smaller by 10 .

However, if no padding is prefixed to the front, then

DataSeries.Pad.Firstoforiginal_int $=1$;

RawSeries.TriggerTime.DateTime_txt = "YYYY-MM-DD hh:mm:ss.sss[z $|+|-] \mathrm{hh}[: \mathrm{mm}]]$ "; DataSeries.TriggerTime.DateTime txt = "YYYY-MM-DD hh:mm:ss.Sss[z|[+|-] hh[:mm] ]";

|| ISO DateTime (preferably to ms) of the TRIGGER time. Not to be

1) confused with the first-sample times, FirstsampleTime.

RawSeries.TriggerTime.TimeError dbl = <value $\rangle$ s;

DataSeries.TriggerTime.TimeError dbl = $\langle$ value $\rangle$ s;

|| Estimated or computed two-sigma error

Optional

Optional

Optional

Optional 
|| Pre-Event buffer length (the amount of the trace

recorded prior to the moment of triggering).

DataSeries.PostDeTriggerDuration_dbl = <value $\rangle$;

|| How long DAU records after de-triggering.

| | Recommended

In modern systems, which only rarely are short of event-storage memory, we recommend a PreTriggerDuration of at least $60 \mathrm{~s}$ and a PostDeTriggerDuration of at least $120 \mathrm{~s}$. While these intervals are significantly larger than typically are used presently, they aid greatly with:

1. baseline removal and integration,

2. ambient-noise characterization, and

3. making it much more likely that rapid sequences of triggered records will overlap, thus allowing unambiguous reconstruction of data from more Events in such a sequence.

There is no longer much, if any practical need for shorter pre- and post-Event intervals. In contrast, their value to analysts is substantial, sometimes critical.

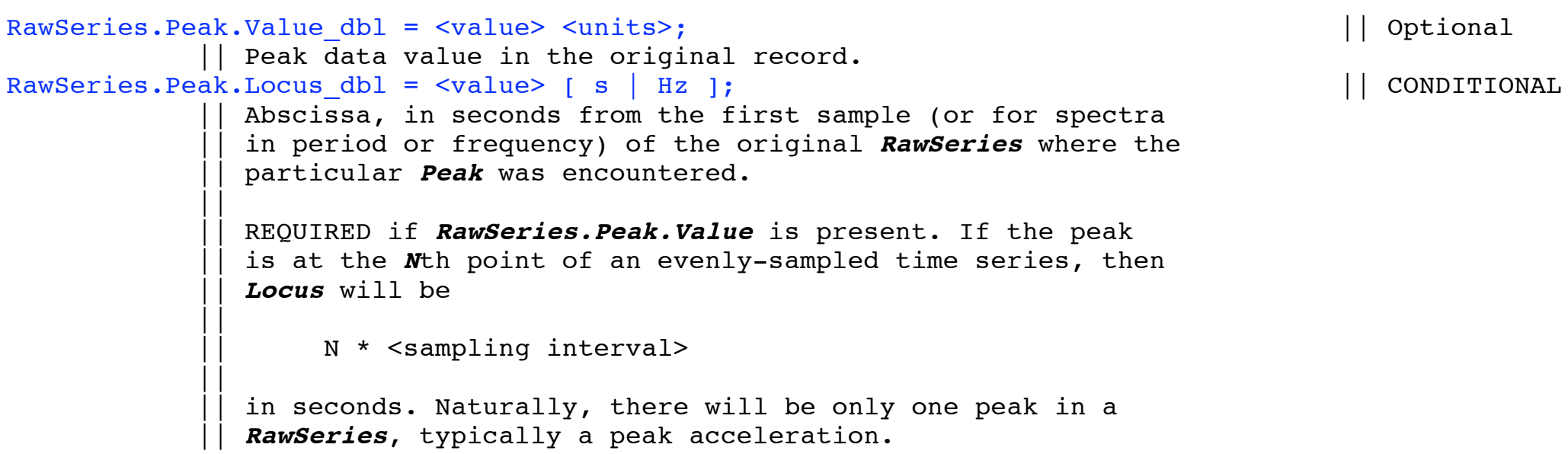

|| Optional

| | CONDITIONAL 
DataSeries.Peak[(n)].Value_dbl = <value> <units>;

Peak data value in this (usually processed) Dataseries. This

| | Recommended

may be a PGA, a PGV, a maximum $\boldsymbol{S}_{a}$, or other measure of shaking

strength, depending on what type of Dataseries this is.

In the case of response spectra, Peak(n).Value is necessarily a tag array of length equal to the size of Dataseries.ResponseSpectrumDamping(n) (that is, there will be one peak value per spectrum, per damping). Use of subscripting, Peak(n), is restricted to that instance since PGA and so forth will have exactly one such value per Dataseries.

DataSeries.Peak[(n)].Locus_dbl = <value $>$ [ s $\mid \mathrm{Hz}$ ];

The location in the Dataseries where

DataSeries.Peak(n).Value was encountered, expressed

either in time (in seconds since first sample) or the period or frequency of the spectral maximum (for Dataseries

which are spectra) of the values (and in the same order).

Thus, if the peak occurs at the Nth point of a Dataseries, this value is $N$ * <sampling rate> for time series or $(\mathrm{N}-1) *$ <bin width> for evenly binned spectra. For unevenly sampled spectra, this value simply corresponds to the frequency of a particular spectral line.

DataSeries.Peak[(n)].ResponseSpectrumPeakTime_dbl = <value> s;

If and only if DataSeries.Peak(n).Value are for response spectra, then also record the TIME in the original time series (seconds since first sample) at which this spectral maximum is encountered. This tag also will be a tag array of length $\boldsymbol{N}$ equal to the size of ResponsespectrumDamping(n), that is one spectrum, one peak, one peak's period, and one TIME in the RawSeries (the accelerogram) where this peak was encountered.

DataSeries.Peak(n).Locus and/or

DataSeries.Peak(n).ResponseSpectrumPeakTime are

REQUIRED if DataSeries.Peak(n).Value is present (both

must be given if the Dataseries is a response spectrum,

otherwise only the former). 
RawSeries.FilmDigitizer.Ystep_dbl = <value> um;

RawSeries.FilmDigitizer.Tstep dbl = <value $\rangle$ um;

RawSeries.FilmDigitizer.Tmean dbl = <value $>\mathrm{s} \mid \mathrm{um}]$;

RawSeries.FilmDigitizer.Tsigma_dbl = <value $>[\mathrm{s} \mid \mathrm{um}]$;

RawSeries.FilmDigitizer.Tminimum dbl $=\langle$ value $\rangle[\mathrm{s} \mid \mathrm{um}]$

RawSeries.FilmDigitizer.Tmaximum dbl = <value $\rangle$ [s um ];

|| If this record derives from a film-recorded Rawseries,

then these tags are the sizes of the digitizer $X-$ (time)

and $\mathrm{Y}$-steps (amplitude), and statistics pertaining to

them, as used in digitizing that film record.

CONDITIONALS are REQUIRED for film-derived records.

DataSeries.ResponseSpectrumDamping(n) dbl = <value ;

| | CONDITIONAL

REQUIRED if the DataSeries are response spectra, $\boldsymbol{S}_{a}, \boldsymbol{S}_{v}, \boldsymbol{S}_{d}$,

PSA, or PSV.

A tag array that is as large as (and in exactly the same order as)

the ordinate columns in the main Dataseries listing matrix. This

tag array gives the value(s) of "structure" damping used for

those calculations. It is fairly common, for example, to provide

response-spectral values at 2-, 5-, and 10-percent structural damping.

Values are in fractions of critical, never in percent. So, for

the above example, the damping would given as $0.02,0.05$, and 0.1

Typically, there also will be an explicit-abscissa column in the DataSeries listing matrix, giving the periods (or conceivably the frequencies). An explicit abscissa is likely because it is almost invariably the case that the sampling periods of the response spectrum are unevenly spaced, often at periods approximating a logarithmic sequence, or at some other irregular spacing. 
A selected subset of linear $S_{a}, S_{v}$ or, $S_{d}$ values also may be given for time series (for $S_{a}$ we recommend periods including 0.2, 0.3, 1.0 and 3.0 s for compatibility with ShakeMap and other processes). These selected values typically are included when the DataSeries is the processed ("volume 2") time series, as a convenience for users and for ShakeMap and similar analyses.

We note that this is an incomplete set of response-spectral tag names - one might desire to include PSA and PSV and both linear and nonlinear versions of any values given here. Please e-mail the authors if this turns out to be an issue-such tags are easily added.

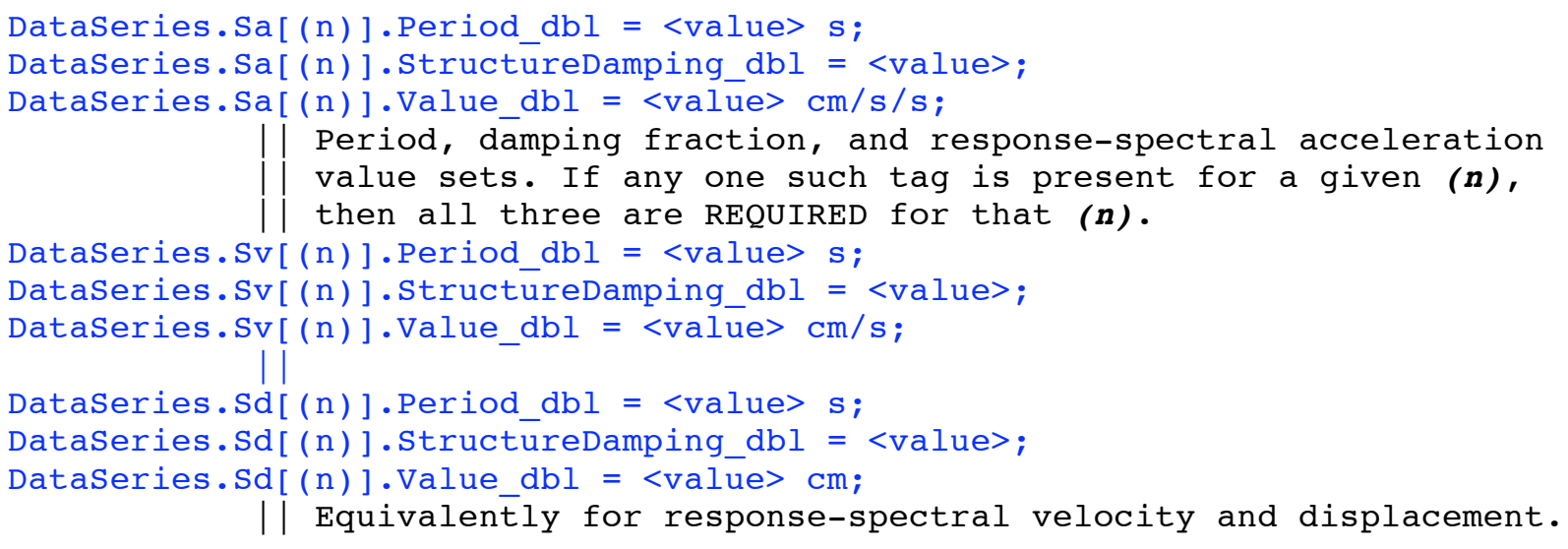

CONDITIONAL CONDITIONAL

CONDITIONAL

CONDITIONAL

CONDITIONAL

CONDITIONAL

CONDITIONAL

CONDITIONAL

CONDITIONAL

Most of the following are meaningful only when this VTF file's DataSeries is a time series.

RawSeries.RMS_dbl = <value> <units $>$;

IT RMS of an original time series

DataSeries.RMS dbl = <value $\rangle$ <units $\rangle$.

|| RMS of this VTF file's Dataseries (a time series)

|| Optional

|| Optional 
DataSeries.CAV_dbl = <value $>[\mathrm{m} / \mathrm{s} \mid \mathrm{cm} / \mathrm{s}]$;

|| Cumulative Absolute Velocity of this time series

DataSeries.SI dbl = <value $>[\mathrm{m} \mid \mathrm{cm}]$;

|T Housner Intensity (SI) of this time series

DataSeries.AriasIntensity_dbl $=\langle$ value $>[\mathrm{m} / \mathrm{s} \mid \mathrm{cm} / \mathrm{s}]$;

|| Arias Intensity of this time series

DataSeries.ObservationalMMI [ ( $)$ ]_int = <value; 1-12>;

|| MMI of this time series, determined by human

inspection of effects at the site. (There may be

more than one observer with differing opinions.)

DataSeries. InstrumentalMMI $\mathrm{dbl}=\langle$ value, $0.0-12.0\rangle$;

|| Optional

|| Optional

|| Optional

|| Optional

| Optional

| Optional

| Optional

| | Recommended

| | Recommended

Dataseries.Pick[(n)].SDateTime txt = "YYYY-MM-DD hh:mm:ss.ss[s][z|[+|-]hh[:mm] ]" :

$\mid \begin{aligned} & S \text {-Wave arrival time Pick(s) at this Geolocation (there may } \\ & \text { be more than one opinion on the time of the pick). }\end{aligned}$

(may, therefore, be noninteger). Generally saturates

at about 10 .

Many practitioners believe that the MMI scale effectively

saturates at intensity $\mathrm{x}, 10$, with all higher values in

not yet "given wisdom".)

please e-mail the authors with a scale name, citation,

DataSeries.Duration.Over5PctG dbl = <value $\rangle$;

| Duration of shäking more than 5 percent of g_standard

Dataseries.Duration.AriasInterval5To95Pct_dbl $=<$ value $>$ s

| Interval to accumulate from 5 to 95 percent of the final Arias

Intensity for this time series.

DataSeries.Pick[(n)].PDateTime_txt = "YYYY-MM-DD hh:mm:ss.ss[s][z|[+|-]hh[:mm] ]";

| P-Wave arrival time Pick(s) at this Geolocation (there may 
DataSeries.Pick[(n)].Agency_txt = "<table 3E $>$ ";

| CONDITIONAL

REQUIRED if either DataSeries.Pick.PDateTime or

DataSeries.Pick.SDateTime is given, and is the group

or individual (Agency from table 3E) that made the pick(s).

DataSeries.Pick[(n)].DateTimeMade_txt = "YYYY-MM-DD [ hh:mm:ss [z|[+|-]hh[:mm] ] "; || CONDITIONAL

Dataseries.Pick.SDateTime is given, and is the date

( \pm time) on which the pick(s) were made (that is, the

pedigree of the Pick(s), not the Pick time itself) to

facilitate tracking updates to the $\boldsymbol{P i c k}(\mathrm{s})$.

RawSeries.Comments txt $=$ "<Comments $>$ ";

Comments about the RawSeries on matters not covered

elsewhere.

Dataseries.Comments txt $=$ "<Comments $>$ ".

Comments about the DataSeries on matters not covered

elsewhere.

These tags are for any Comments about the Dataseries or

RawSeries not otherwise covered by defined Dataseries or

RawSeries tags, or simply to hold a comment that the

providing Agency considers important.

If multiple "lines" of text are needed, join them into

a single text string, with the line breaks indicated by

the character string "/nn". 


\section{Table 3M. Recorder Timing Method}

The following values are used for RawSeries.FirstSampleTime.Source and DataSeries.FirstSampleTime.Source.

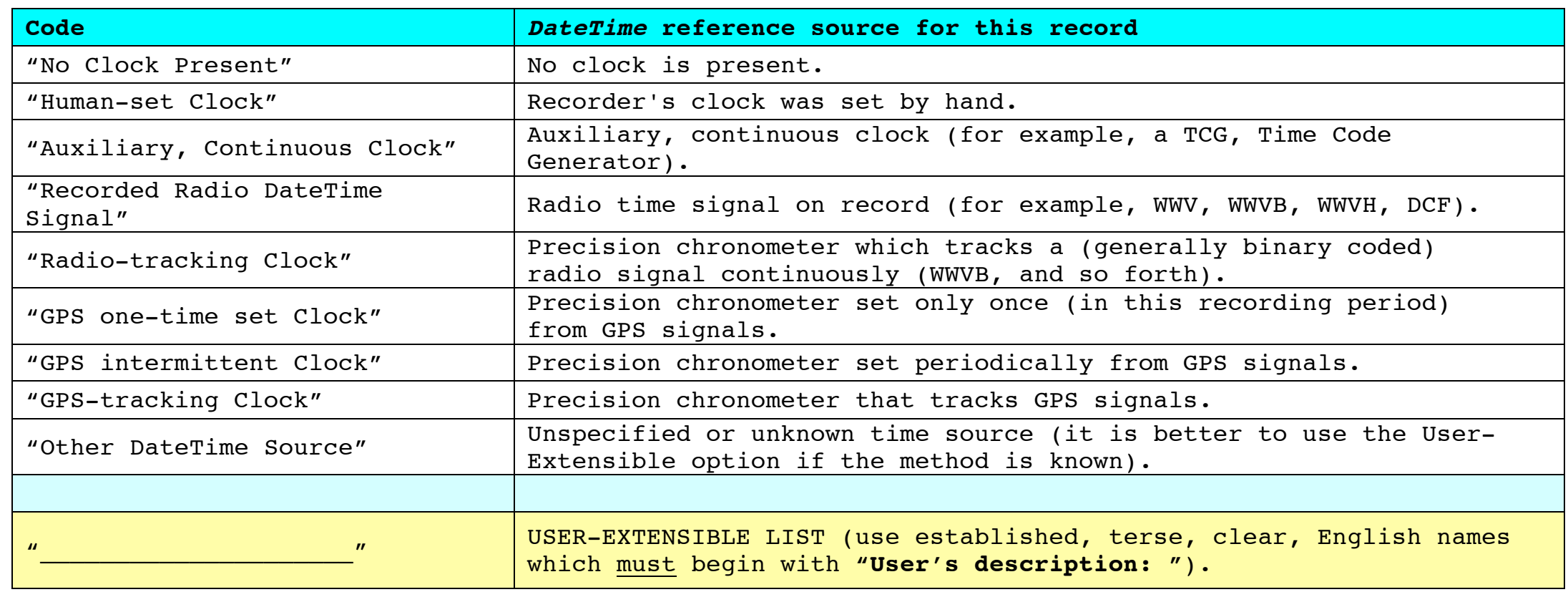




\section{Table 3N. Processing Information}

Processing.DateTime txt $=$ "YYYY-MM-DD hh:mm:ss.sss [Z|[+|-]hh[:mm]

Authoritative ISO DateTime when the record was processed

| | CONDITIONAL

to its final form (that is, the form in this VTF file).

This tag-value pair is REQUIRED if this is not an

unprocessed RawSeries (thus, nearly always).

Note that Processing.DateTime identifies the originating-

Agency's authoritative creation DateTime for the DataSeries in

this file, while Thisfile.Preparation.DateTime is the DateTime

when this VTF file is created (often by means of translation from

the originating-Agency's format).

Clearly, therefore, Processing.DateTime must not be later

than Thisfile.Preparation.DateTime, but it is possible for

these two DateTimes to be the same if the originating-Agency

directly creates this VTF file.

Processing.Agency_txt = "<value $>$ ";

Processing organization or individual (Agency from table 3E).

| | CONDITIONAL

If said Agency also provides the file in VTF, this value likely

will be the same as ThisFile.Preparation.Agency.

REQUIRED if Processing.DateTime is present (which is nearly

always).

Processing.Filter information is REQUIRED if one or more Filters are applied during Processing. Furthermore, if any Processing.Filter tag is given, then a "sufficiently descriptive" set of such tags is REQUIRED (enough that another practitioner can determine what was done and its affect on the DataSeries).

If there is more than one Filter, Subscript them as Processing.Filter(m)...; if only one Processing.Filter is used, the Subscript may (should) be omitted and will default to "1". 
Note that there are three methods for describing a filter-poles and zeros (PoleZero), Polynomial equivalents of those poles and zeros, and much less complete, general parameters (OtherDescription) such as corner frequencies and roll-off rates. The PoleZero and Polynomial forms are preferred, the former above the latter. Also note that there are a number of global Processing.Filter tags which can be applied to any Filter.

In this order, we describe Filter global tags, PoleZero tags, Polynomial tags, and OtherDescription tags:

The following group of global tags apply to all three types of Filter description:

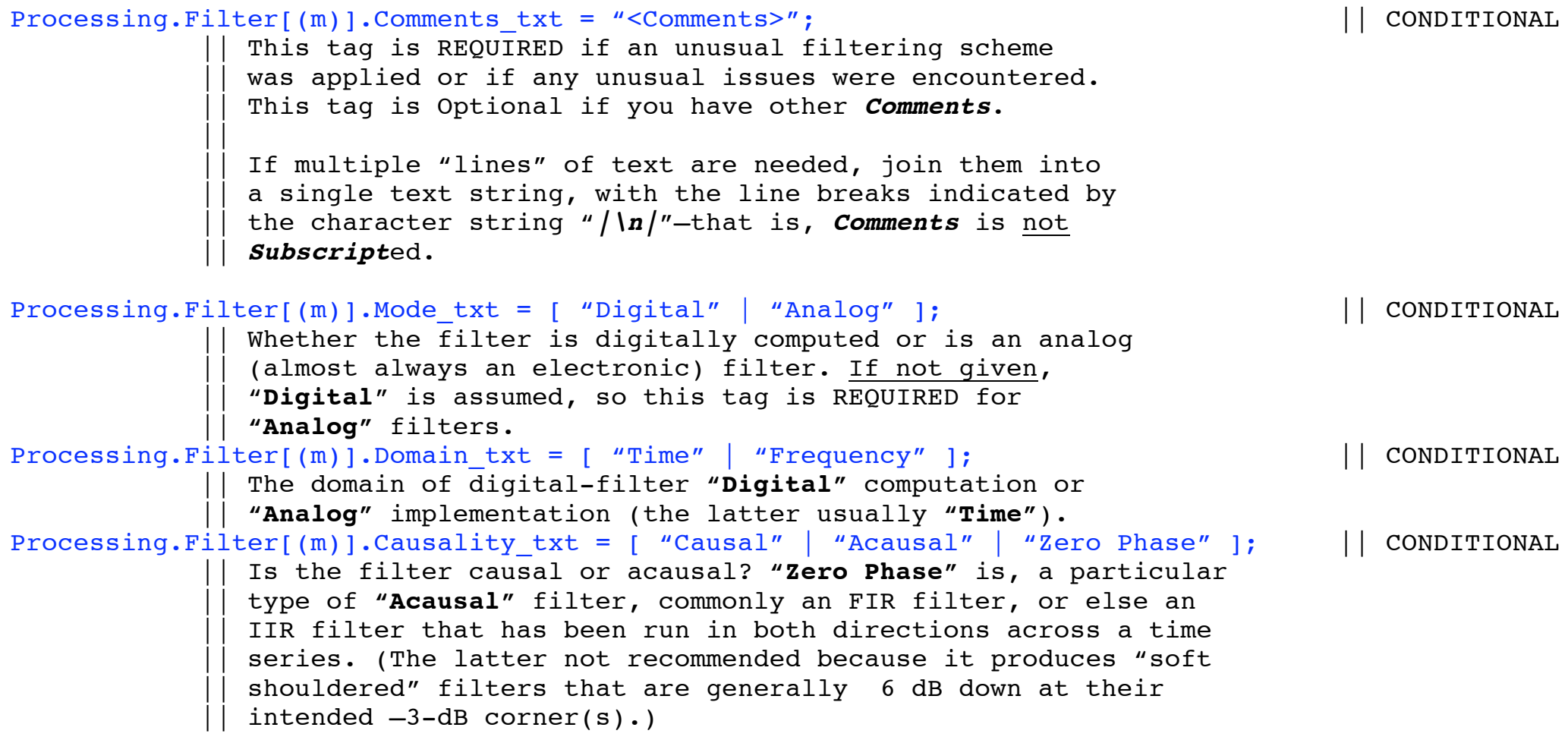


Processing.Filter $[(\mathrm{m})]$. FrequencyBandType_txt $="\langle$ from table $30(\mathrm{~b})\rangle$ ";

Processing.Filter $[(\mathrm{m})]$.PeriodBandType_txt $="<$ from table $30(\mathrm{~b})>$ ";

| Filter bandpass character (for example, "High Pass") from table $30(\mathrm{~b})$.

Most scientists are likely to use FrequencyBandType, while many structural engineers may prefer to use PeriodBandType.

(This distinction between types of abscissa, seconds or Hertz, matters only to the meanings of "Low Pass" and "High Pass"

filters. The distinction is at which end of the passband

that filter corner resides. Thus, for example, is it

described by a "high-pass period" or a "high-pass

frequency".)

Processing.Filter $[(\mathrm{m})]$.FilterName_txt $=$ "<from table $30(\mathrm{a})>$ ";

The common name of this Filter.

For Polezero or Polynomial descriptions, use only one

of the following:

"S-plane Poles/zeros"

"z-plane Poles/zeros"

"S-plane Polynomial"

"z-plane Polynomial".

For otherDescription, use one of the common names of the Filter

such as "Ormsby" (a zero-phase cosine-tapered bandpass filter-

the name "Ormsby" is not commonly used outside strong-motion

seismology and earthquake engineering).

Naturally, for a Polezero description, only the values

"S-plane Poles/zeros" or "z-plane Poles/zeros" from

table $30(\mathrm{a})$ are allowed.

Similarly, for a Polynomial description, only the values

"S-plane Polynomial" or "z-plane Polynomial" from table $30(\mathrm{a})$

are allowed. 
Poles and zeros, particularly Laplace ( $S$-plane) poles and zeros, are the preferred method, with Polynomials being the next most desirable method. An exception, of course, is that it will generally be preferable to describe an FIR filter by its z-plain Polynomial.Numerator coefficients (which are the same as the filter's "taps").

If using a PoleZero representation, then a sufficient set of the following six tags is REQUIRED. Either the Numerator or the Denominator of a PoleZero description may have no roots and therefore be only a constant (which will often be just a "1"). In all cases, a Constant may be given for that portion (Numerator or Denominator), but will default to a real value of one (1) if not provided - often the desired result. (Constant is given type _cpx for generality, but we expect it to be real in most cases; remember that if you do not give the imaginary part, it will default to $0 j$, a real). The corresponding NumberOfRoots will be zero and no Roots will be listed for that portion. Where there are roots and the Constant =1; the Constant will typically will be omitted, defaulted to a real-value of one (1). This commonly will be the case for filters with no net gain:

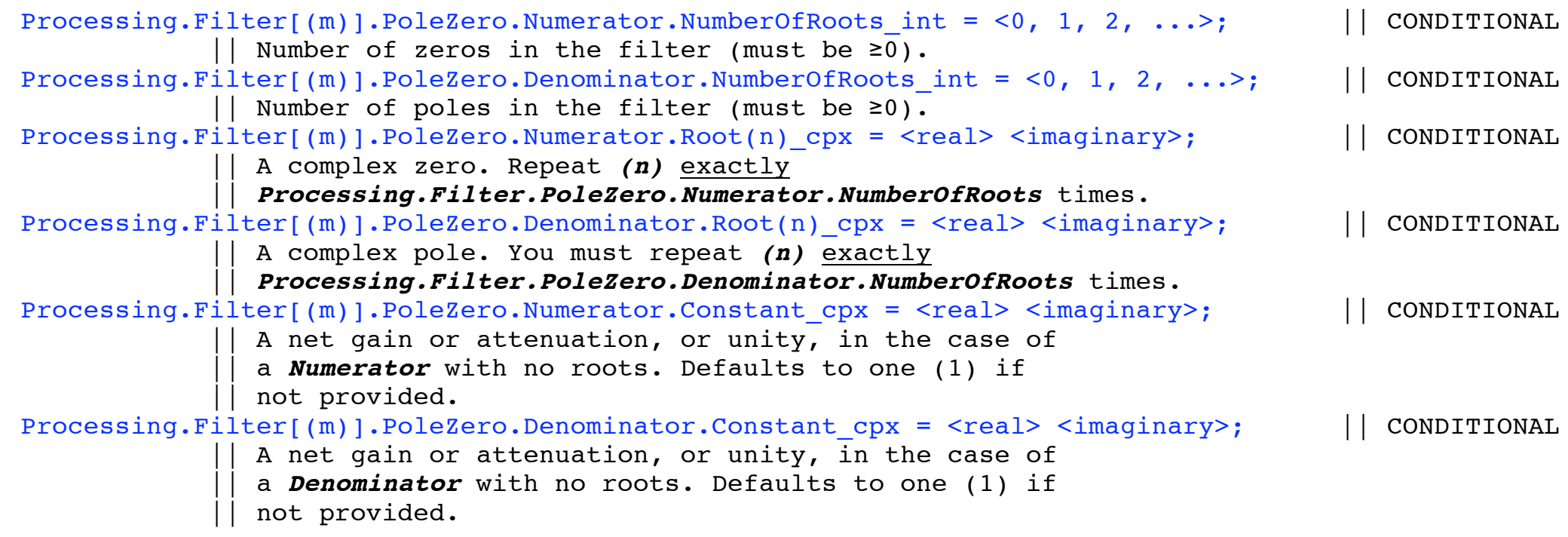


Polynomials are close relatives of pole/zero descriptions, being the enumeration of polynomial coefficients for the Numerator and Denominator of a filter transfer function, simply the PoleZero form multiplied out. These may be in the Laplace- $(S)$ or z-plane, as indicated by Sensor.TransferFunction.FilterName, the former preferred.

The order (maximum power) of the Polynomial is $\mathbf{N}=$ NumberOfCoeff $-\mathbf{1}$. The ordering of the Coefficients is from 0-to-N, corresponding to Subscripts 1-to-NumberOfCoeff. Thus, these Coefficients are given in ascending order from the constant, $\boldsymbol{n}=1$, up to $\boldsymbol{n}=\mathbf{N u m b e r O f C o e f f}=N+1$, the Nth-degree coefficient. Any zero-valued Coefficient also must be given explicitly to create a complete vector of NumberOfCoeff elements (some applications will be confused by missing Coefficients).

If either the Numerator or Denominator has no roots, then the value of Coefficient(1) will act as a simple gain factor while NumberOfCoeff will equal one (1). This is a somewhat different rule than for the PoleZero form where the number of poles or zeros may be zero (0), but a Constant is always present, even if defaulted to unity (1).

Processing.Filter $[(\mathrm{m})]$.Polynomial.Numerator.NumberofCoeff_int $=\langle 1,2,3, \ldots>$, Processing.Filter $[(\mathrm{m})]$.Polynomial.Denominator.NumberOfCoeff $f$ int $=<1,2,3, \ldots>$; || Both must be $\geq 1$.

Processing.Filter $[(\mathrm{m})]$.Polynomial.Numerator.Coefficient(n) $\mathrm{cpx}=\langle\mathrm{value}>$;

Processing.Filter $[(\mathrm{m})]$.Polynomial. Denominator.Coefficient $(\overline{\mathrm{n}})$ _cpx $=\langle\operatorname{value}\rangle$;

CONDITIONAL CONDITIONAL

CONDITIONAL CONDITIONAL 
If not using either the PoleZero or Polynomial forms, the following tags are another way (in combination with Processing.Filter.FilterName) to describe processing filters, albeit less generally or accurately. A sufficiently complete group of these tags is REQUIRED if neither poles and zeroes nor polynomial coefficients are given, but a filter was used.

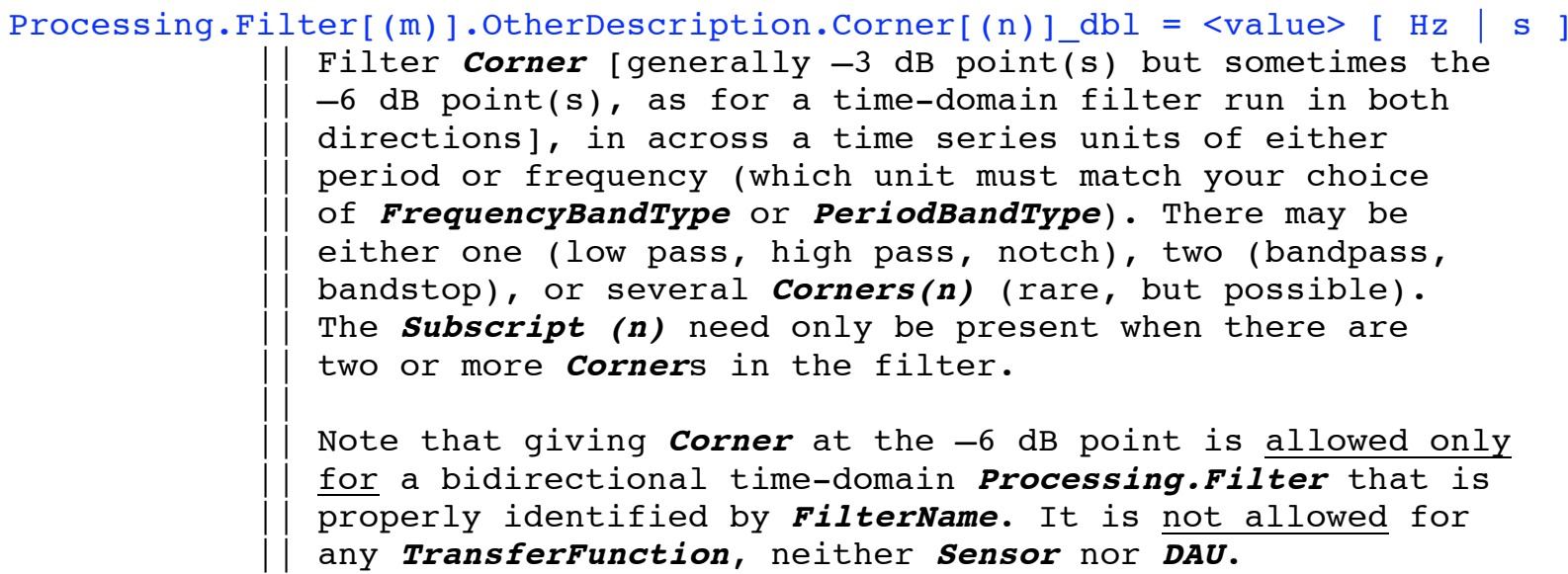

Processing.Filter [(m)].OtherDescription.TransitionBandwidth_dbl = $\langle$ value $\rangle$ Hz; Processing.Filter $[(\mathrm{m})]$.OtherDescription.StopBandAttenuation $\mathrm{dbl}=\langle\mathrm{value}\rangle \mathrm{dB}$;

|| Needed for Ormsby filter, FIR brickwall filters, and 
Processing.Filter[(m)].OtherDescription.Length_dbl = <value> s;

| CONDITIONAL

Length of filter operator, even if symmetric, when doing

time domain filtering (for example, FIR, IIR). For an FIR, this

value often is called the "number of taps" although a few users define "taps" as $<1+(($ Length -1$) / 2)>$, which is

the least number of multiply-accumulate operations required

for a symmetric (zero phase) FIR filter, notable for their

symmetric, odd-length impulse responses.

Note that this value is in seconds for generality.

Processing.Resampling[(m)]. InitialSamplingRate_dbl $=<$ <ld sample rate> $\mathrm{Hz}$;

Processing.Resampling $[(\mathrm{m})]$.FinalsamplingRate dbl $=<$ new sample rate $>\mathrm{Hz}$;

Processing.Resampling [ (m) ].FilterSubscript_int $=<$ index $>$;

Legacy data were sometimes decimated to reduce computation

times and storage requirements (back when computers were much

less capable than they were in 2008). Similarly, some modern data

occasionally are up-sampled to make identification of peak values

and arrival times easier and more accurate.

The first two tags are REQUIRED if this DataSeries was either decimated or up-sampled, with both values being samples per second; the pair, therefore, implying the up- or down-sampling

factor.

If desired, users may employ Processing.Filter values to describe resampling filter(s) more completely. If you do this, make it clear with the corresponding Comments and Filtersubscript which Processing

filter is, in fact, the Resampling Filter (that is, it will be

Processing.Filter(Processing.Resampling.Filtersubscript). So

the third tag is REQUIRED when using Filter to describe Resampling.

The index (m) can be used to describe multiple steps in

decimation and/or up-sampling. For example, some legacy CSMIP

data first decimate by four then by an additional amount for

computing long-period results. This would be described

with (m), (1) being the initial decimation. 
The following four tags relate to double-integration initial conditions. Also see DataSeries.Pad.FirstOfOriginal and DataSeries.Pad.LastOfOriginal.

RawSeries.Mean_dbl = <value><units>;

|| Mean value removed to make the previous time series

| in this Processing path zero-mean.

DataSeries.Mean_dbl = <value> <units>;

|| Mean value of this time series.

| | Recommended

| | Recommended

Processing.Report[(n)].Citation_txt = "<citation>";

Processing.Report $[(\mathrm{n})]$. URL txt $=$ "<Web address $>$ ";

| If there is/are report(s) available for this Dataseries,

describing its Processing, provide a citation with one or

both of these tags.

Processing.Initialvalue.Velocity_dbl = <value $><$ units $>$;

Processing. Initialvalue.Displacement_dbl = <value $\rangle\langle$ units $\rangle$;

Often it is necessary to begin an integration (of acceleration

to velocity, or velocity to displacement) from an initial value

other than zero in order to produce a trace with is found in

this VTF file as the Dataseries. The tag Displacement, for

example, is the constant of integration and corresponds to

"remembering" the acceleration baseline initial correction

needed to integrate the RawSeries into either this VTF file's

Dataseries, or into an intermediary velocity signal.

Processing.Initialvalue.AccelerationCorrectionRecord_txt = "<name of file or ID>"; This third tag points to some more general description of baseline corrections (including the fact that an offset (mean) correction in velocity is a Dirac delta in acceleration, an offset correction in displacement is the derivative of a Dirac delta in acceleration, and so forth). That is, AccelerationCorrectionRecord points to a providing-Agency's filename, VTF filename, or unique identifier for a time series in which the Dataseries.Physicalparameter is effectively "Acceleration Correction Baseline". 
Corrections made prior to integration routinely include such delta functions (typically convolved with a smoothing filter for convenience). Smoothed baseline-correction time series also regularly include by one or more higher-order corrections (such as a piecewise-linear velocity baseline, which translate into an acceleration step). Thus, the most complete description of a baseline correction is a complete acceleration time series of the same length and sampling rate as the (commonly padded) acceleration RawSeries.

(We do not now include velocity-correction equivalents because these are subsumed with the acceleration correction. However, it will be desirable to add such tags whenever the VTF begins to be used for primary velocity records, such as those of the Tokyo Sokushin VSE-355G3.)

Processing.Constantsused_txt $=$ [ "Precise" | Nominal" | "Poor" ];

Describes the accuracy of conversion and correction constants used in correcting and Processing the seismogram. The list of possible values is from best to worst, with "Nominal" meaning that up to a few percent total gain and phase error is possible in the pass band, while "Precise" means that no more than 1 -percent total error is likely.

Processing.Problem[(m)].Status_txt = [ "None" | "Problem of Unknown Status" | "Corrected"| "Not Corrected" | "Presence of Problem Uncertain" ]

If no issues with data Processing are known to have existed at any time, then "None" (that is "No Problems") is given. If problems with the Dataseries are known to be, or to have been, present at any time, then you must include at least one Processing.Problem.Comments(n) tag explaining what the problems are or were. Such problems are either "Corrected" or "Not [yet] Corrected". "Problem of Unknown Status" may be used when the correction status is unknown, but it is believed there has been a problem. "Presence of Problem Uncertain" just means there is neither exculpatory nor indicting knowledge. 
The Subscript (n) allows the listing of more than one Problem, but will often be only an implicit (1).

Processing.Problem [ (m) ].Comments [ ( $)]$ txt $=$ "Description";

| | CONDITIONAL

| If Processing.Problem.status is anything other

than "None", then this tag is REQUIRED for each (n)

to describe the nature of the problem(s) and indicate

their current disposition.

If multiple "lines" of text are required for any

particular (n), then concatenate them into a single

text string with the lines separated by the character

string " $/ \ln \mid "$.

Processing. Comments txt $=$ "<Comments $>$ ";

| | Recommended

| For general Comments about Processing that are not

appropriate to Processing.Problem.Comments or

Processing.specialprocessing.

As above, for multiple "lines" of text in any particular Comments(n), concatenate them into a single text string with the lines separated by the character string "||n|".

Processing.BlueBookVolume int $=[0-3]$;

Processing.Stage txt $=$ [ "Raw" | "Preliminary"| "Final" ];

Processing.HumanReview_txt = [ "None" | "Reviewed" | "Updated" ];

Processing.Instance int $=$ <positive integer>;

Processing.Specialprocessing txt = "<Description $>$ ";

One of the two tags Processing.BlueBookvolume or

Processing.Stage is required to indicate the stage

of the Processing. Furthermore, within each Processing

stage, there may be one or more instances of the release

of a DataSeries, indicated by Processing.Instance. The

latter is REQUIRED, but will often be exactly 1 , the

first and only such Processing instance. 
It also is REQUIRED that the degree of human analysis of the data (versus automated computer Processing) be indicated. If the data are, so far, only processed automatically then set Processing.HumanReview_txt = "None"; (This often will be the case with the initial release of data from modern, digital systems.) At subsequent steps in the Processing scheme, use the other values to indicate that the data have received a preliminary review by a human, or that they have been processed fully and subjected to full quality-control procedures. Legacy and older data generally are predominantly human-processed and are, therefore, given the value Processing.HumanReview_txt = "Updated" ; in most cases. (We are not so optimistic as to offer the value "Final".)

Processing.BlueBookVolume takes the values:

0 = unprocessed data

1 = converted and baseline-corrected ground motion

2 = filtered acceleration, velocity, or displacement

$3=a$ spectrum or spectra, including response spectra

Finally, if the DataSeries has been processed in a manner that is not typical of the originating Agency, indicate this fact by including the tag Processing.specialprocessing

(REQUIRED when there is unusual processing) and using its

contents to describe what was unusual about the Processing.

\section{NOTES:}

(1) Channel numbers and sensing directions are now in table $3 \mathrm{~K}(\mathrm{a})$.

(2) All geographic-location information is now in tags defined in table 3F. 


\section{Table 3O(a). Codes for Filter Types Used in Processing}

Table 3O(a) describes the possible values of Processing.Filter(n).FilterName. The first four values also may be used for Sensor.TransferFunction.FilterName or Sensor.TransferF unction. FilterName.

Note that the causality of filters are indicated by the formal definitions of those filters and by Processing.Filter.Causality and DAU.TransferFunction.Causality, while Sensor transfer functions are assumed to be causal. This may pose a conflict for Sensors packaged with digitizers, but it should be possible to differentiate the two systems - we take the position that a digitizer, even when packaged inside the Sensor, is part of the $\boldsymbol{D A U}$, and the appropriate descriptive tags will be found there.

\begin{tabular}{|c|c|}
\hline Phrase & Any FilterName \\
\hline "S-plane Poles/Zeros" & A list of complex Laplace-plane poles and zeros follows. \\
\hline "z-plane Poles/Zeros" & 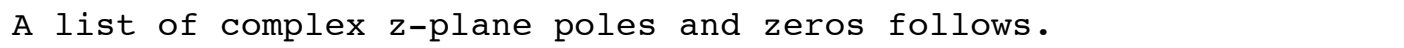 \\
\hline "S-plane Polynomial" & A list of real Laplace-plane polynomial coefficients. \\
\hline "z-plane Polynomial" & A list of real z-plane polynomial coefficients \\
\hline \multicolumn{2}{|c|}{ (the following not allowed for pole-zero descriptions) } \\
\hline "None" & No filtering performed (just leave out the variables entirely) \\
\hline "Rectangular" & Rectangular \\
\hline "Cosine Bell" & Cosine Bell \\
\hline "Ormsby" & Ormsby \\
\hline "Butterworth" & Butterworth, single direction (state "Causal" or "Acausal" please). \\
\hline "Bessel" & Bessel, single direction (state "Causal" or "Acausal" please). \\
\hline
\end{tabular}




\begin{tabular}{|l|l|l|}
\hline “IIR" & $\begin{array}{l}\text { Generalized time- } \\
\text { domain IIR filter. }\end{array}$ & It is best to treat these as z-plane poles and \\
& $\begin{array}{l}\text { FIR (state } \\
\text { filter's } \\
\text { causality). }\end{array}$ & zeros. \\
\hline “Other Transfer Function" & Use for DAU or Sensor TransferFunctions not described formally. \\
\hline “Other Processing Filter" & Any other Filter response not listed above. \\
\hline “ & $\begin{array}{l}\text { USER-EXTENSIBLE LIST (use established, terse, clear, English names } \\
\text { which must begin with "User's description: "). }\end{array}$ \\
\hline
\end{tabular}

\section{Table 3O(b). Codes for Filter Types Used in Processing}

Similarly, table 3O(b) shows the values that Processing.Filter(n).FrequencyBandType or Processing.Filter(n). PeriodBandType may take.

\begin{tabular}{|l|l|}
\hline Phrase & Filter FrequencyBandTye or PeriodBandType \\
\hline “High Pass" & A high-pass filter, with respect to frequency or to period. \\
\hline “Low Pass" & A low-pass filter, with respect to frequency or to period. \\
\hline “Bandpass" & A band-pass filter. \\
\hline “Bandstop" & A band-stop filter. \\
\hline “Notch" & A notch filter. \\
\hline "Deconvolution" & A transfer-function deconvolution filter. \\
\hline " & $\begin{array}{l}\text { USER-EXTENSIBLE LIST (use established, terse, clear, English names } \\
\text { which must begin with "User's description: "). }\end{array}$ \\
\hline
\end{tabular}




\section{Table 3P. Relational Tags Involving Two Geographic Locations}

EventGeoLocation.EpicentralDistance.Value dbl $=\langle$ value $\rangle \mathrm{km}$;

EventGeoLocation.EpicentralDistance.ValueError_dbl = <value $\rangle \mathrm{km}$;

|| Epicenter-to-Sensor GeoLocation distance and error.

EventGeoLocation.ForeAzimuth.Value_dbl = <value $\rangle$ deg;

EventGeoLocation.ForeAzimuth.ValueError_dbl $=\langle$ value $\rangle$ deg;

|| Epicenter-to-Sensor GeoLocation azimuth and error.

EventGeoLocation.BackAzimuth.Value_dbl = <value $\rangle$ deg;

EventGeoLocation.BackAzimuth.ValueError dbl = <value $\rangle$ deg;

|| Sensor GeoLocation-to-Epicenter azimuth and error.

EventGeoLocation.Comments txt = <value>;

Comments, including any needed identification of which

Event and Geolocation are being combined. We currently

do not have an elegant or unique way to do this.

If multiple "lines" of text are required, then

concatenate them into a single text string, with

the lines separated by the character string "/|n|".

GeoLocationRupture [(m)].ClosestRuptureDistance.Value_dbl = <value $\rangle \mathrm{km}$;

GeoLocationRupture [(m) ].ClosestSurfaceDistance.Value dbl = <value $\rangle \mathrm{km}$;

GeoLocationRupture $[(\mathrm{m})]$.ClosestSeismogenicDistance.Value_dbl $=\langle\mathrm{value}\rangle \mathrm{km}$;

GeoLocationRupture $[(\mathrm{m})]$. RMSdistance.Value_dbl $=\langle$ value $\rangle \overline{\mathrm{km}}$;

GeoLocationRupture [(m)].SegmentDistance.Segment(i).Value dbl $=\langle$ value $\rangle \mathrm{km}$;

$$
\text { || Values and ... }
$$

GeoLocationRupture [(m)].ClosestRuptureDistance.ValueError dbl $=\langle$ value $\rangle \mathrm{km}$;

GeoLocationRupture [ (m) ].ClosestSurfaceDistance.ValueError dbl $=\langle$ value $\rangle \mathrm{km}$;

GeoLocationRupture $[(\mathrm{m})]$.ClosestSeismogenicDistance.ValueError_dbl $=\langle\mathrm{value}\rangle \mathrm{km}$;

GeoLocationRupture $[(\mathrm{m})]$.RMSdistance.ValueError dbl $=\langle$ value $\rangle$ km;

GeoLocationRupture [(m)].SegmentDistance.Segment(i).ValueError_dbl $=\langle\mathrm{value}>\mathrm{km}$; their errors.

Optional

Optional

Optional

Optional

| Optional 
Sensor GeoLocation-to-Fault-Rupture (and -to-Rupture-Surface-

Projection) closest distances. Such values are often used in

ground-motion model calculations). Subscripts (m) and (i) refer

to a particular rupture model and must be the same as the subscripts

in Event.DetailedFaultRupture(m).Segment(i).Latitude and related tags.

Note that ClosestSurfaceDistance is the Boore and others (1993, 1994

1997) distance, ClosestSeismogenicDistance is the Campbell (1997)

distance, and ClosestRuptureDistance is "ClstD" in the NGA flatfile.

The first four tags are distances either to a simple, one-segment rupture model, or to a rupture taken as a whole. In contrast, the fifth tag is for distances to each segment of a modeled rupture

surface area:

\section{GeoLocationRupture(m).SegmentDistance.Segment(i).Value}

Of course, any of these tags can be computed from the

Sensor's Geolocation together with the rupture description

in Event.DetailedFaultRupture.Segment(i) and, clearly, should

be consistent.

GeoLocationRupture $[(\mathrm{m})]$. SurfaceRupture_txt =

$$
\text { [ "Yes" T"No"| "Inferred" | "Unclear" ]; }
$$

| Optional

Whether or not this Event has an associated surface rupture.

(If nothing is known about surface ruptures, you may set the

value to NULL, or leave out the tag.)

(While this and the next two tags might be more appropriately

located in class Event, we believe there is a significant need

to make explicit the correlation with other GeolocationRupture

tags.)

GeoLocationRupture [ (m) ].SurfaceRuptureInference_txt = "<value $>$ "

|| The reason for any inference of surface rupture. 
The "CONDITIONALs" mean that once a TagName is created, for any (n), all other CONDITIONALs must be present too, including the DataType, data Units (NULL if unitless, but remember that a unit "counts" is available and meaningful), and the individual or organization (Agency) creating that Private tag. The Value to which this tag is set must be included too, noting that every Value shall be encoded as text, including numerical data. TextValueError need not be given.

"Avoid" means the Private-tag class generally should not be necessary and will be hard for others to use-try not to invoke it.

We have attempted to anticipate all user needs by providing appropriate tags, however, we expect the need for additional options will arise. Thus, Private tags may be used for quality-control information, data not yet supported by the VTF, specialized network data, and so forth, assuming that using some already-available Comments instead is not an acceptable alternative.

The Private-tag mechanism is one way in which new tags may be proposed to COSMOS, although a more formal proposal is preferred (that is, please contact one of the authors).

VTF parsers will treat all Private tags as simple text when read for Processing, and they will be passed through unchanged, as viable tag sets, within the data files themselves. That is, DataType is advisory, suggesting how to parse the Value. (Your own parser may read them in, and use them as, particular types of values. Since the VTF source code will be available to all users, it should be a relatively simple programming job to modify parsers, output generators, and translators to read and write your Private tags.) 


\section{Table 3R. DataSeries Tags Closely Associated with Table 4}

DataSeries.NumberOfSamples int $=$ <number of samples in the DataSeries>;

|| REQUIRED

Number of samples in time (or bins in frequency, or periods), regardless of whether the interval between samples, frequency bins, or periods is regular or irregular. This value must equal the number of rows (lines) in the Dataseries listing matrix between "DataSeries.NumberofSamples_int $=\{$ " and " $\}$;", the sense being "the number of values per damping value" in the case of response spectra. Any zero-value-abscissa bin is included in this count.

It is also the number of lines in any ASCII listing accessed through any of the DataSeries.Remote...URL_txt tags. There is no provision at present for differing formats or line counts between DataSeries.DataSeriesvalues and a remote file. So if the local and remote copies of the DataSeries differ in size or format, only one should be provided. The same is true of DataSeries.Ordinateunits and a few related tags.

DataSeries.OrdinateUnits [ (m) ] txt = "<units $>$ ";

A list of units for the ordinate(s) in the Dataseries listing matrix (from table 2), one index for each ordinate column in the Dataseries listing matrix. There will be as few as one set of units in the case of any DataSeries except response spectra. (The <units> for the abscissa are given by Dataseries.AbscissaUnits.)

These units must conform to the type of data (from

table 3B(b)) indicated by Dataseries.Physicalparameter.

DataSeries.OrdinateFormat $[(\mathrm{m})]$ _txt $=$ "<FORTRAN format specification $>$ ";

| | REQUIRED REQUIRED whenever the Dataseries is provided, either here in this VTF file or at a URL as an ASCII file.

The Fortran-style format(s) for the ordinate portion(s) of the Dataseries listing matrix. 
Where there are multiple-ordinate column vectors in the ordinate, the format of each and every ordinate column (except for complex values) is indicated by a separate $(\boldsymbol{m})$. That is, there are no repetition counts in these formats.

(The exception is that, because there must be two columns for every complex-valued ordinate, it is important that each such real/imaginary pair associate to a single (m). That is, each format for a complex value is given as an concatenated pair of formats in a single string, and that it must include the requisite white space between the real and imaginary columns.)

In the case where both this VTF file and a URL to an ASCII file are present, this single ordinateformat must apply to both. That is, at present, the VTF does not have a way to express different ordinateFormats (or AbscissaFormats) in local and remote ASCII Dataseries listings.

While the ordinateformat defined here might be expected to include an entry for the abscissa column-vector's format, that role is filled by DataSeries.AbscissaFormat.

Most often, the Dataseries listing matrix will have exactly one value per line, a single column of real values. This will

be true of all real, evenly sampled time series. Thus there

most often will be a DataSeries.OrdinateUnits(1) and a

DataSeries.OrdinateFormat (1).

\section{Critical Notes on Formats}

Authors are cautioned to provide enough text precision in every format to preserve the precision of the original recording, with no conversion or round-off errors. (They should, therefore, have a mantissa at least as precise as the analog-to-digital converter [ADC] and generally somewhat longer). For 24-bit records in $\mathrm{cm} / \mathrm{s} / \mathrm{s}$, for example, try "F11.5". For related complex spectra try "E16.9 E16.9". Over-specifying is not recommended because it may imply greater precision than is available from the data, but under-specifying is worse, damaging the data. 
In all DataSeries listing matrices it is essential that the AbscissaFormat and OrdinateFormat(s) you specify be wide enough to ensure (1) that there will always be at least one blank space between each value, and (2) that columns also will align Fortran style. This rule is necessary to ensure the coexistence of older languages like Fortran and newer ones like C, MatLab ${ }^{\mathrm{TM}}$, and Excel $^{\mathrm{TM}}$. That is, the VTF requires both column alignment and white-space separation in the DataSeries listing matrix.

Response spectra generally will have both an abscissa column (a real-valued period or frequency) plus exactly as many real-valued ordinate column vectors as there are damping values in DataSeries.ResponseSpectrumDamping(n). Thus, there will be $N+1$ columns in the DataSeries listing matrix, while both DataSeries.OrdinateFormat(n) and DataSeries.OrdinateUnits $(\boldsymbol{n})$ must have the same dimensions and order as ResponseSpectrumDamping(n).

Tags for remote DataSeries (those external to this VTF file):

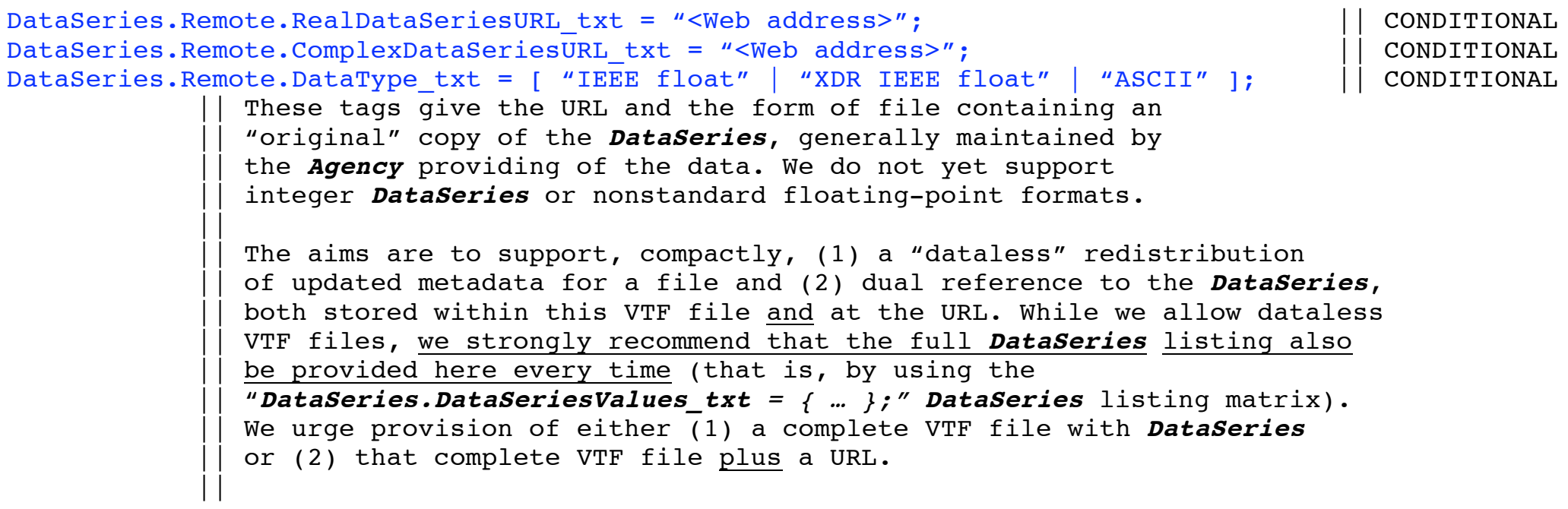


One of the first two tags is REQUIRED if (1) this is not a "dataless" file and (2) the Dataseries is not provided below, in this file (by "DataSeries.DataSeriesvalues_txt $=\{\ldots\} ; "$, the DataSeries listing matrix). Otherwise, these tags are Optional and may be provided in addition to the Dataseries listing within this file, as desired.

Again, we do not recommend either dataless or URL-only usage since a key goal of the VTF is to present, in s single file, a complete Dataseries with all its supporting metadata.

The third tag, DataSeries.Remote.DataType, is REQUIRED whenever one of the first two tags is given. "IEEE float" implies that the data found at that URL will be in the provider's local flavor of IEEE double or IEEE complex double (whichever is implied by this VTF file). "XDR IEEE float" indicates that the data are in the "XDR" (external Data Representation) version of those formats (cf. below).

Other, machine dependent, floating-point formats and binary-integer formats are not supported at this time.

"ASCII" indicates that the data are text in exactly the same format used for the Dataseries listing matrix, just as presented in this VTF file. That is, it must use exactly the same ordinateFormat and AbscissaFormat as in this VTF file. There is currently no provision for differing ASCII formats between this file and any remote "ASCII" file.

XDR encoding of IEEE floating-point values is strongly recommended (that is, DataSeries.Remote.DataType_txt = "XDR IEEE float";), simply because XDR-binary format is well-established and fully portable between computers of differing architectures and, therefore, is easily translated between them. XDR input and output subroutines also exist for every architecture supporting NFS file systems, which includes

all versions of UNIX and, therefore, every major computer architecture in use). 
DataSeries.Remote.BytesToSkip_int $=<$ bytes $>$;

DataSeries.Remote.LinesToSkip_int $=\langle$ lines $>$;

These tags describe how far to skip into the file to get beyond

any preamble (for example, a header) in that remote file. (This skip distance can be described in bytes for IEEE-float files or

in lines for ASCII files). Since the goal is to use the VTF header given above, header information in the remote file must be skipped (there is no provision to read remote header information from VTF software). Further, a concatenated remote file can be handled by defining the correct number of samples and how far into the remote file to skip before reading.

(Note that we do not recommend concatenating files for various DataSeries anywhere, since these can be difficult for users to parse or sort. There is no longer any reason to save a few bytes by such concatenation.)

Whichever of these two tags fits the type of file at the URL, that tag is REQUIRED whenever the first byte of that remote file is not the first character of the Dataseries. (So, as mentioned, use BytesToskip for binary files and LinesToskip for ASCII files. )

Both default to zero if not given.

DataSeries.Checksum int $=\langle$ value $\rangle$;

Optional checksum for the Dataseries listing matrix only, that listed in "DataSeries.DataSeriesValues_txt $=\{\ldots\} ;$ ".

This checksum is computed as follows: sum all the mantissa and exponent digits, if any, (first subtracting five from each digit so that the values summed range from -5 to +4$)$; add to this sum each sign, valued as +1 for each positive and as -1 for each negative (these without first subtracting five, so values of either \pm 1$)$; to this sum, add nothing for each NaN.

(Note that this value must be recomputed every time any change is made to the Dataseries listing matrix, including to any of its formats.) 


\section{Table 4. The Time Series or Spectrum (the DataSeries Values)}

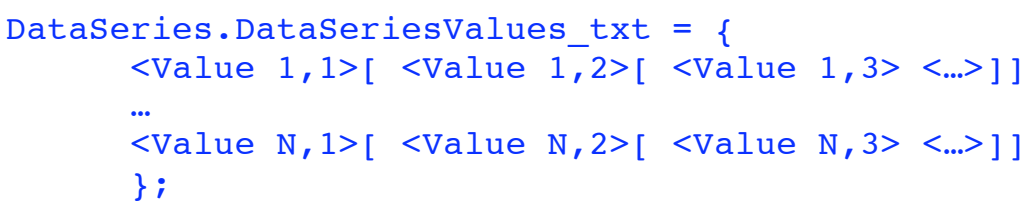

where "N" is the number of lines in the DataSeries and, therefore, must equal to DataSeries.NumberOfSamples. The "f" and "f;" are DataSeries listing "matrix" (or "vector" for a single, real value) delimiters.

Although DataSeries.DataSeriesValues indicates type "_txt", the values will be parsed, as appropriate, into "int", "_dbl", "_cpx", or a mix of these.

We recommend that the DataSeries be provided here in the VTF file in addition to any URL pointer to an Agency original and even when only the metadata have changed and one might be tempted to distribute a "dataless" file.

If the first line is present, the entire DataSeries listing matrix is REQUIRED, as well as the closing line, "\};". Avoid blank lines within the DataSeries listing matrix, though they are allowed.

Notwithstanding this recommendation, the DataSeries listing matrix is allowed be provided only by way of a URL. Similarly, a VTF file is allowed to be "dataless" (that is, containing only a header and neither a DataSeriesValues nor a URL pointing to the original DataSeries); in this case, this VTF file is intended only for use in the distribution of revised header metadata applicable to a DataSeries previously disseminated with the same ThisFile.DataSeriesCOSMOSidentifier (analogous to a "dataless SEED” file as used within the CISN, for example). 
The one or more columns of the DataSeries value listing matrix go between " $\{\mathrm{nn}$ " and " $\ \mathrm{n}\} ;$ ", to the exclusion of all comments and preferably to the exclusion of blank lines. (Here "In" means "newline" or "line break", not printable characters in the DataSeries listing matrix.)

Missing values must be replaced by "NaN" ("Not a Number") which parses in most systems into the equivalent IEEE value "NaN". In rare systems "NaN" will have to be replaced by some extremal value that is then announced to the user reading in the DataSeries. Default $\mathrm{NaN}$ value replacements may be suggested to the parser with ThisFile.NullComplexValue, ThisFile.NullRealValue, or ThisFile.NullIntValue.

Common forms of DataSeries listing matrix include, but are not limited to the following:

(1) A real-valued time series will form a single-column matrix (a vector):

<amplitude>

where time begins at DataSeries.FirstSampleTime.DateTime and constantly increases, either in steps of (1 / DataSeries.SamplesPerSecond) or DataSeries.SampleInterval, or as given in an explicit abscissa, hence

$<$ time> <amplitude>

where <time $>$ is seconds from DataSeries.FirstSampleTime.DateTime, and the value of the first element of the <time $>$ vector usually will be zero.

(3) Regularly-binned spectra (for example, a DFT or PSD spectrum) without an explicit abscissa will form either one or two columns (for real or complex spectral values):

<amplitude>

or

<real> <imaginary> 
where the abscissa is taken to be equal-width frequency or period bins (the latter rare) with the first centered at DataSeries.FirstBinFrequency or DataSeries.FirstBinPeriod and proceeding to positive abscissa values in steps of DataSeries.FrequencyBin or DataSeries.PeriodBin. The longest-period bin, while it could be "Inf s" period, need not correspond to $0-\mathrm{Hz}$, nor to the first finite frequency of a DFT, nor any other particular value. It is worth noting that a spectrum regularly sampled by period will not have an abscissa that is the inverse of a DFT's frequencies, since the latter tend to be spaced evenly in frequency, so hyperbolically in period.

For irregularly-binned spectra (or whenever an explicit abscissa is desired) the DataSeries matrix listing will form two or three columns (assuming the abscissa is real-valued), either:

\section{[ <frequency> | <period> ] <amplitude>}

or

$$
\text { [ <frequency> | <period> ] <real> <imaginary> }
$$

where $<$ frequency $>$ and $<$ period $>$ refer to bin centers and must be in ascending order of the abscissa.

(5) Response spectra (for example, $S_{a}$ ) typically will be irregularly sampled and therefore form two or more columns (assuming a real-valued abscissa):

[ <frequency> | <period> ] <amplitude(Damping 1)> [ <amplitude(Damping 2)> [...]]

where $<$ frequency $>$ and $<$ period $>$ refer to (structural) resonator natural frequencies or periods and where exactly one of the response spectral amplitudes must be given for each damping value given in the tag array DataSeries.ResponseSpectrumDamping $(\boldsymbol{n})$ - that is, there would be $N+1$ columns. 


\section{References}

Aki, K., and Richards, P. G. 1980. Quantitative Seismology, Theory and Methods: San Francisco, W. H. Freeman and Company.

Boore, D.M., and Akkar, S., 2003. Effect of causal and acausal filters on elastic and inelastic response spectra, Earthquake Eng. Struct. Dyn., 32, 1729-1748.

Boore, D.M., Joyner, W.B., and Fumal, T.E., 1993. Estimation of response spectra and peak accelerations from western North American earthquakes: an interim report, U.S. Geological Survey Open-File Report, 93-509, 72 p.

[http://pubs.er.usgs.gov/usgspubs/ofr/ofr93509] (last accessed 14 October 2008).

Boore, D.M., Joyner, W.B., and Fumal, T.E., 1994. Estimation of response spectra and peak accelerations from western North American earthquakes: an interim report, part 2, U.S. Geological Survey Open-File Report, 94-127, 40 p. [http://pubs.er.usgs.gov/usgspubs/ofr/ofr94127] (last accessed 14 October 2008).

Boore, D.M., Joyner, W.B., and Fumal, T.E., 1997. Equations for Estimating Horizontal Response Spectra and Peak Accelerations from Western North American Earthquakes: A Summary of Recent Work, Seism. Res. Lett., 68, no. 1, 128-153.

Basöz, N., and Kiremidjian, A.S., 1995. Prioritization of Bridges for Seismic Retrofitting, Technical Report NCEER-95-0007, Multidisciplinary Center for Earthquake Engineering Research, Buffalo, New York.

Basöz, N., and Kiremidjian, A.S., 1996. Prioritization of Bridges for Seismic Retrofitting, Technical Report No. 118, John A. Blume Earthquake Engineering Center, Civil Engineering Department, Stanford University, Stanford, California.

Bray, J.D. and Rodriguez-Marek, A., Geotechnical site categories, Proc. First PEER-PG\&E Workshop on Seismic Reliability of Utility Lifelines, San Francisco, CA, August, 1997.

California Governor's Office of Emergency Services, 2004, Data standardization guidelines for loss estimation-Populating inventory databases for HAZUS 99, $114 \mathrm{p}$.

Campbell, K.W., 1997. Empirical near-source attenuation relationships for horizontal and vertical components of peak ground acceleration, peak ground velocity, and pseudo-absolute acceleration response spectra, Seism. Res. Lett., 68, no. 1, $154-179$.

Choy, G.L., and Boatwright, J.L., Global patterns of radiated seismic energy and apparent stress, J. Geophys. Res., 100, no. B9, $18,205-18,228,1995$.

Dana, P.H., 1997. http://www.colorado.edu/geography/gcraft/notes/datum/dlist.html, University of Texas, Austin. (last accessed 07 Oct 2008).

ISO 8601:2004, Data elements and interchange formats-Information interchange-Representation of dates and times, http://www.iso.org/iso/iso_catalogue/catalogue_tc/catalogue_detail.htm?csnumber=26780. (Last accessed 08 October 2008.)

Hanks and Kanamori, 1979. A moment magnitude scale, J. Geophys. Res., 84, no. B5, 2348-2350.

Gutenberg, B., and Richter, C.F., 1956. Earthquake magnitude, intensity, energy and acceleration, Bull. Seis. Soc. Am., 46, $105-145$. 
IASPEI, 2005. Summary of magnitude working group recommendations on standard procedures for determining earthquake magnitudes from digital data, http://www.iaspei.org/commissions/CSOI.html. (last accessed 07 Oct 2008)

Lee, W.H.K., Bennet, R.E. and Meaghu, K.L., 1972. A method of estimating magnitude of local earthquakes from signal duration, U.S. Geological Survey Open File Report 72-223, p. 28. [http://pubs.er.usgs.gov/usgspubs/ofr/ofr72223] (last accessed 14 October 2008).

Richter, C.F., 1935. An earthquake magnitude scale, Bull. Seis. Soc. Am., 25, no. 1, 1-32.

Nuttli, O.W., 1973. Seismic wave attenuation and magnitude relations for Eastern North America, J. Geophys. Res., 78, no. 5, p. 876885.

NIBS, 2002. Earthquake loss estimation methodology, HAZUS-99 service release 2 (SR2) Technical Manual, prepared by the National Institute of Building Sciences for the Federal Emergency Management Agency, Washington, D.C.

Shakal, A.F., Huang, M.-J., Rojahn, C., and Poland, C., 2002. Current building instrumentation programs and guidelines, in Proceedings Invited Workshop on Strong-Motion Instrumentation of Buildings, Consortium of Organizations for Strong-Motion Observation Systems (COSMOS), 14-15 November, 2001, Emeryville, California, 5-14.)

Wills, C.J., Petersen, M.D., Bryant, W.A., Reichle, M.S., Saucedo, G.J., Tan, S.S., Taylor, G.C., and Treiman, J.A., 2000. A siteconditions map for California based on geology and shear wave velocity, Bull. Seism. Soc. Am., 90, S187-S208. 


\section{Index of Tags}

Array

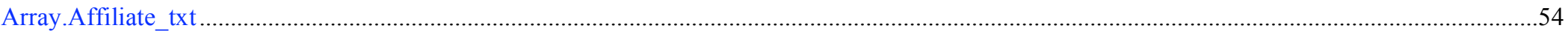

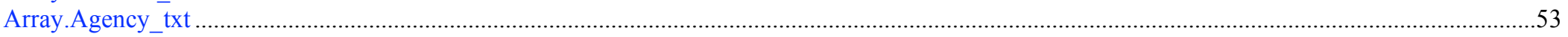

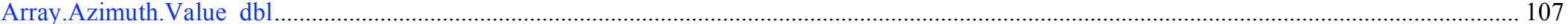

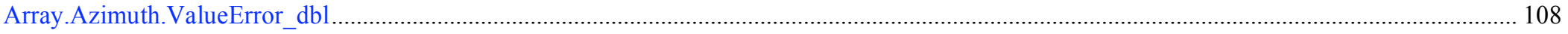

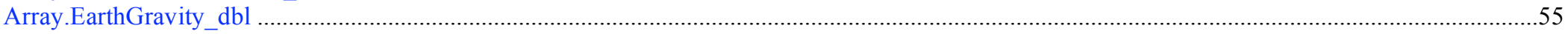

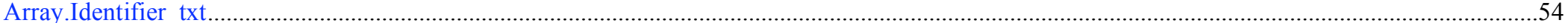

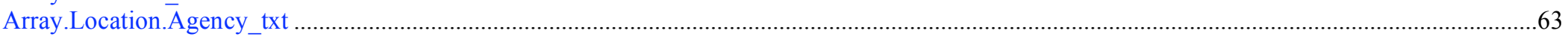

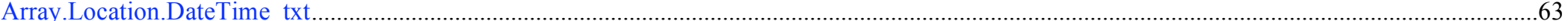

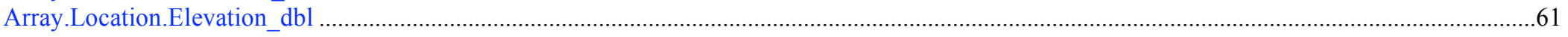

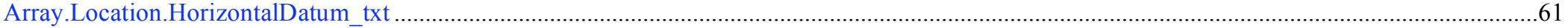

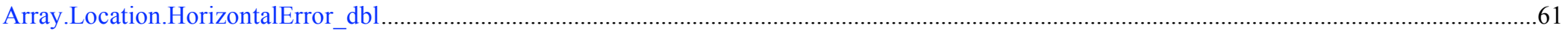

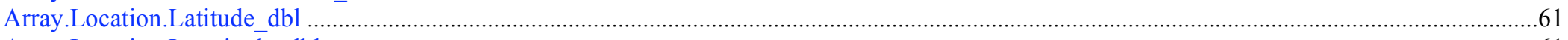

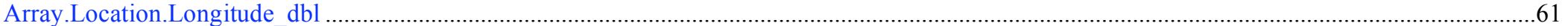

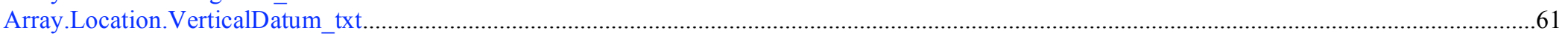

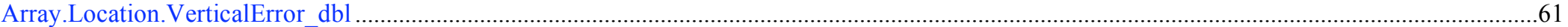

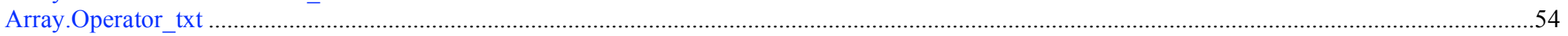

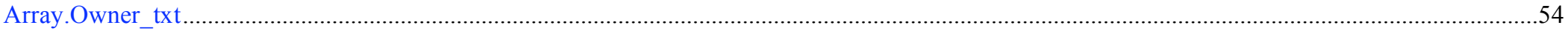

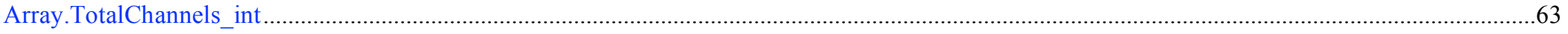

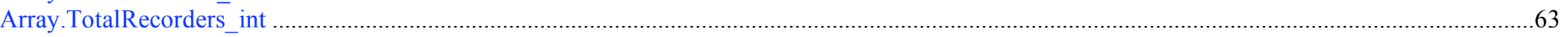

C

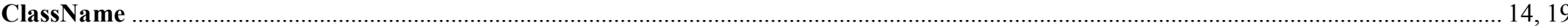

DataSeries

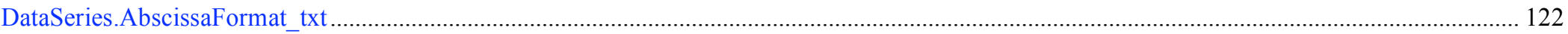

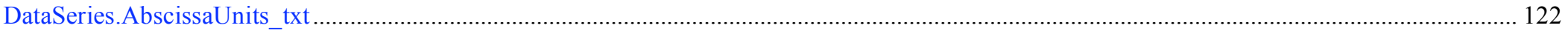

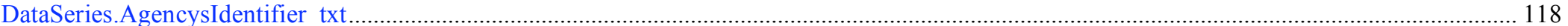

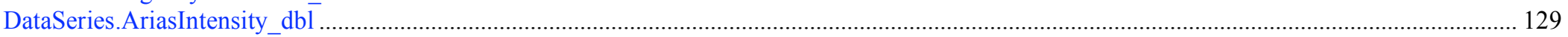

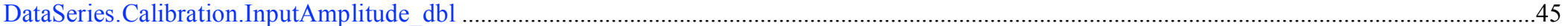

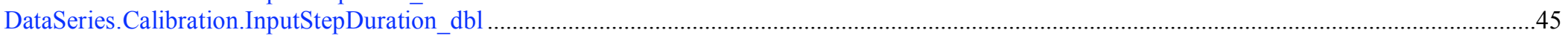




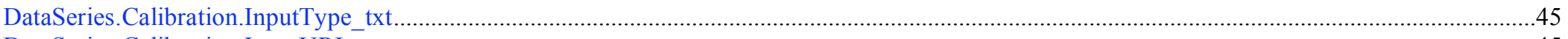

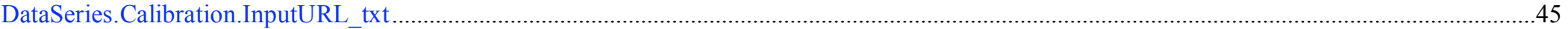

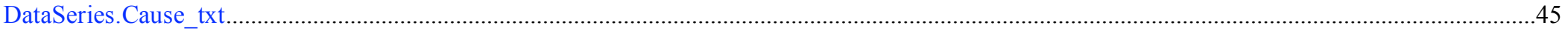

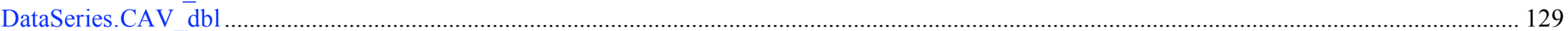

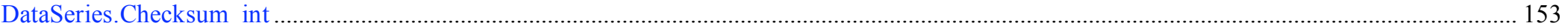

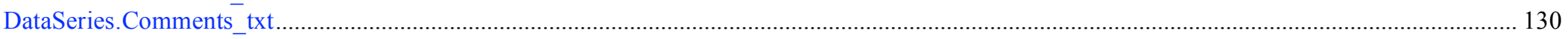

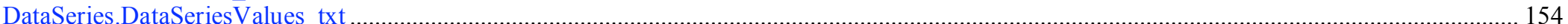

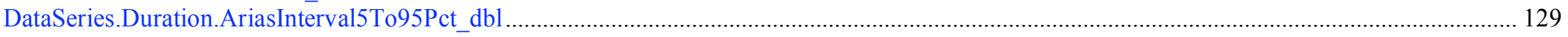

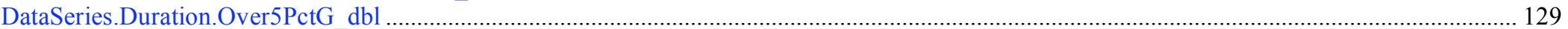

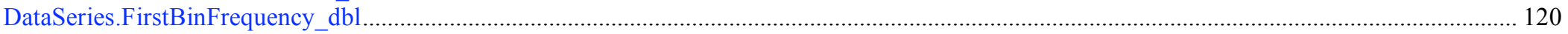

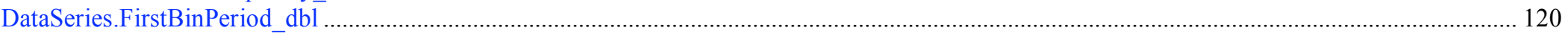

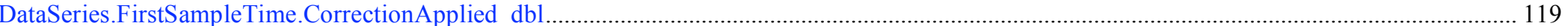

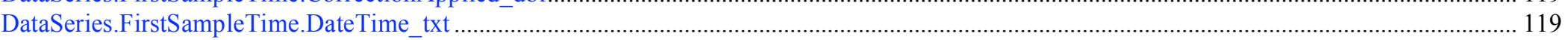

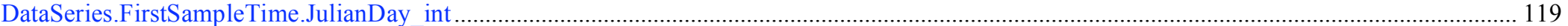

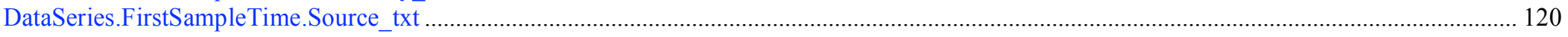

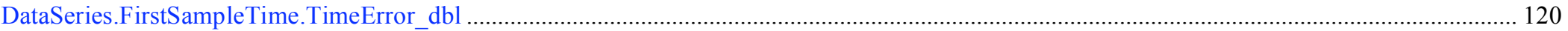

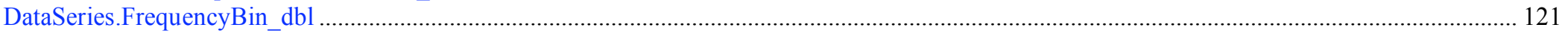

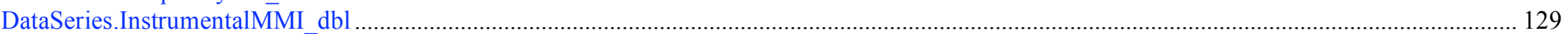

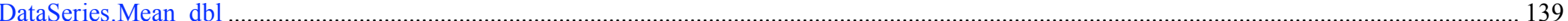

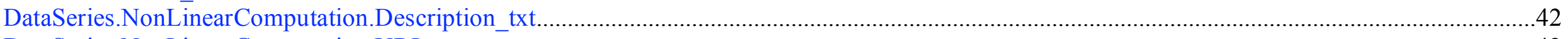

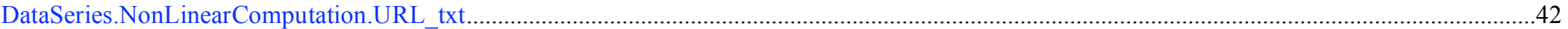

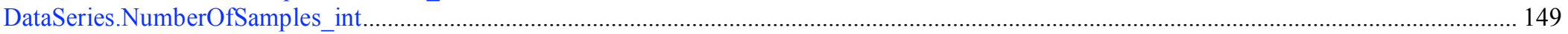

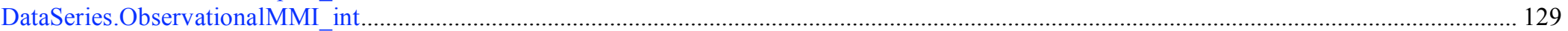

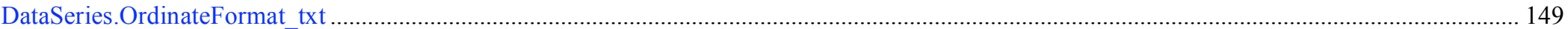

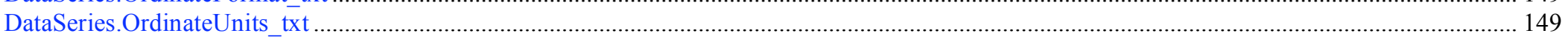

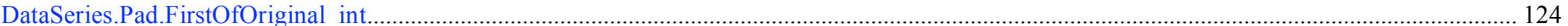

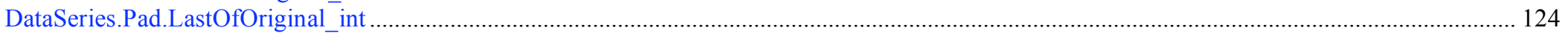

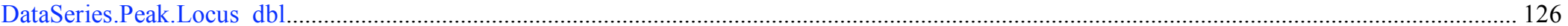

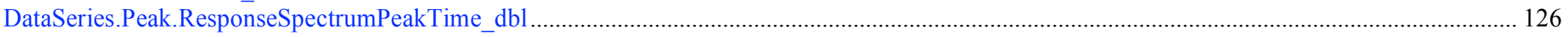

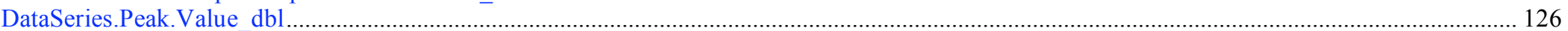

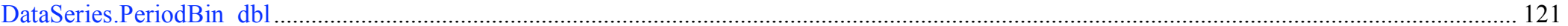

DataSeries.PhysicalParameter_txt

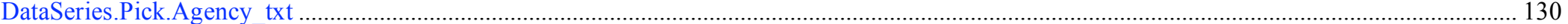

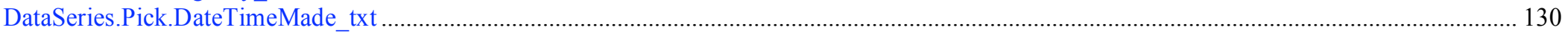

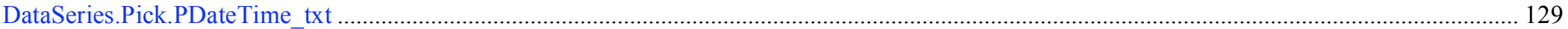

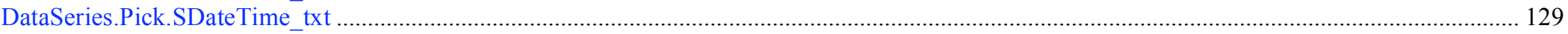

DataSeries.PreTriggerDuration dbl 


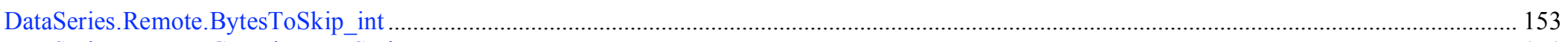

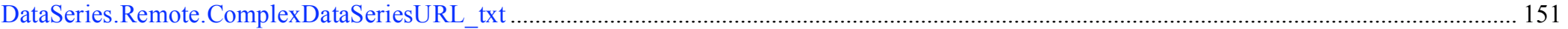

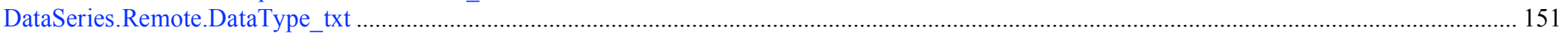

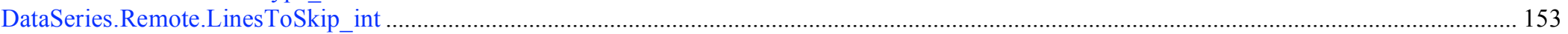

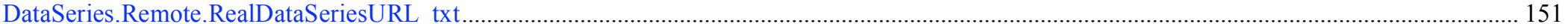

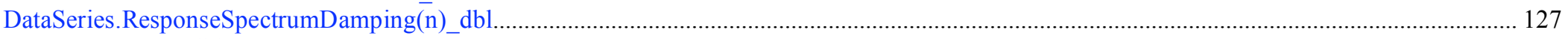

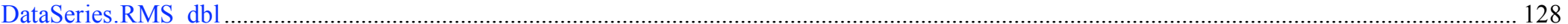

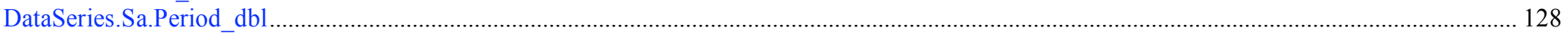

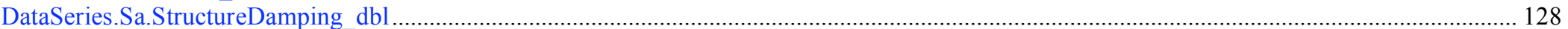

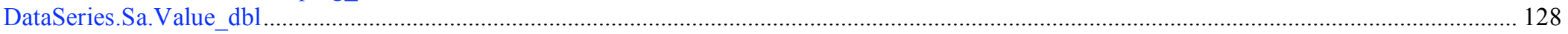

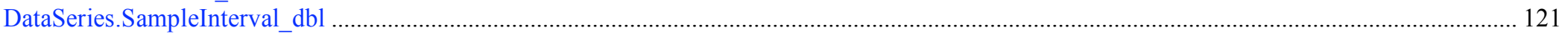

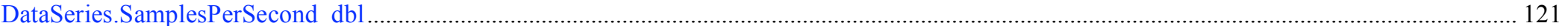

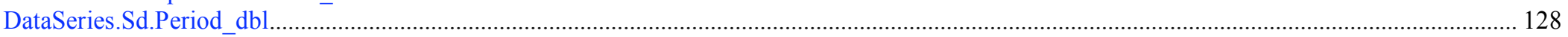

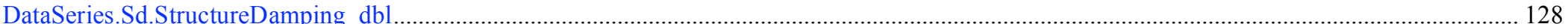

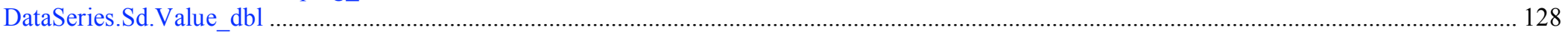

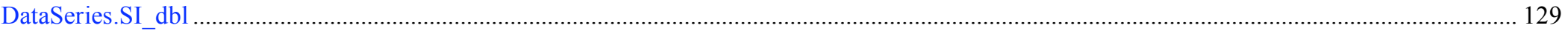

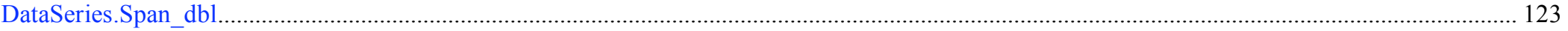

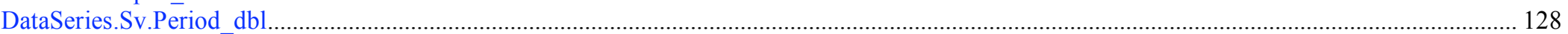

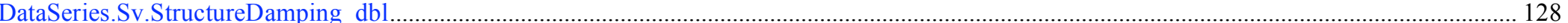

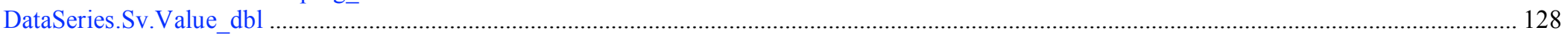

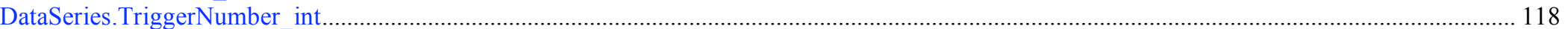

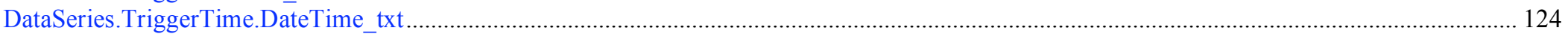

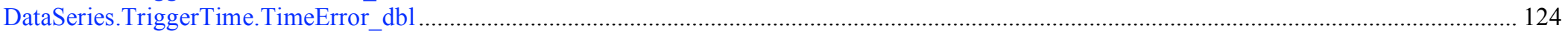

$D A U$

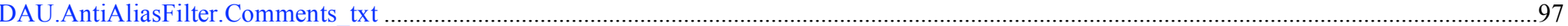

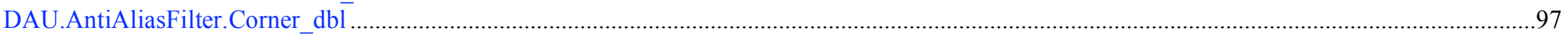

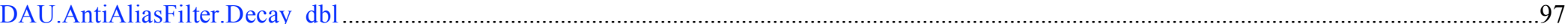

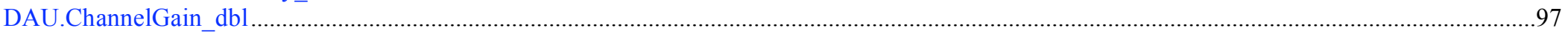

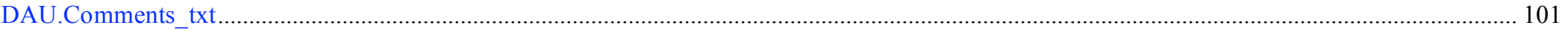

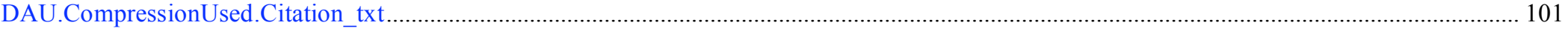

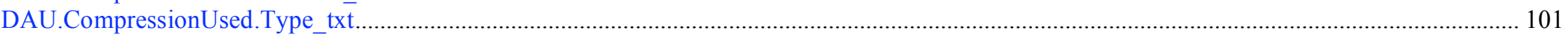

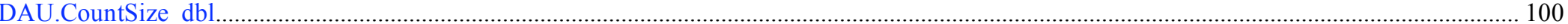

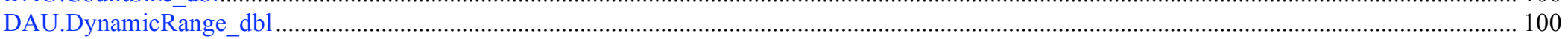

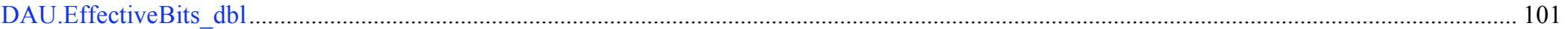

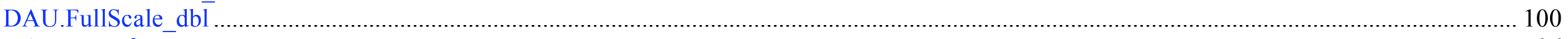

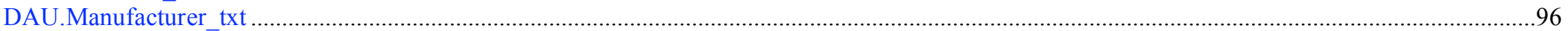




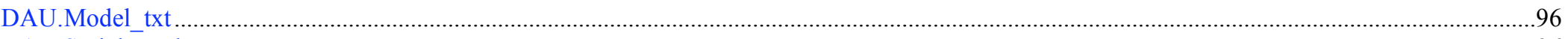

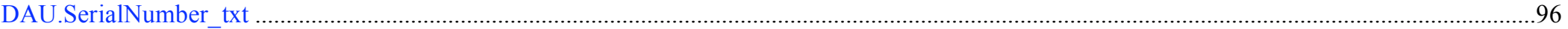

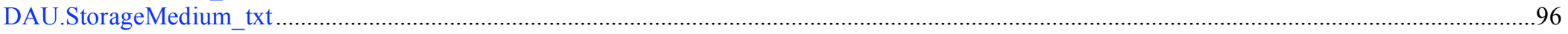

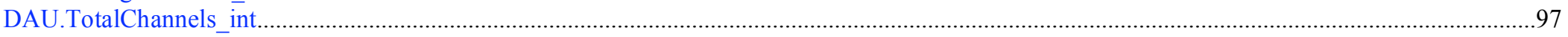

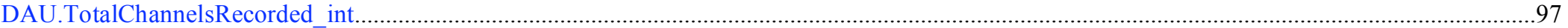

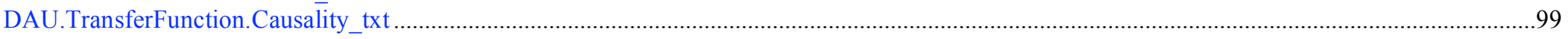

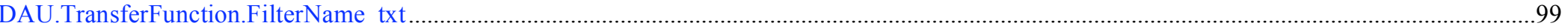

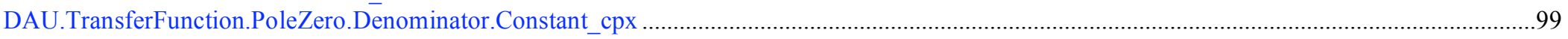

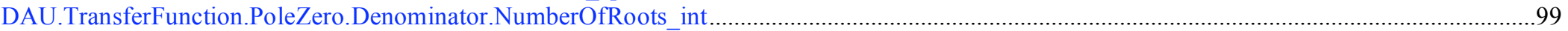

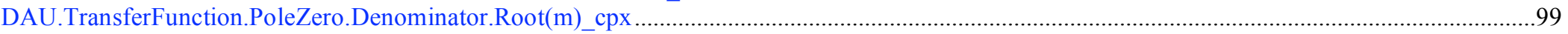

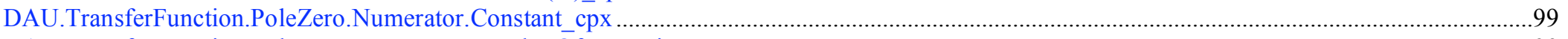

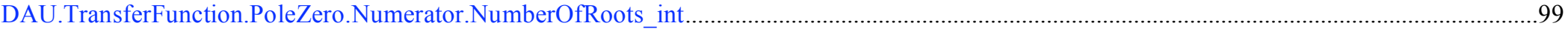

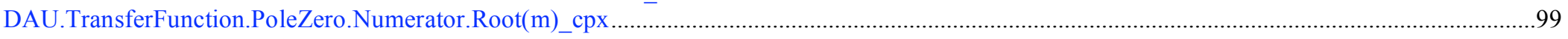

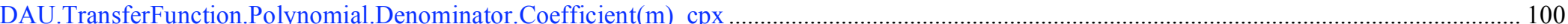

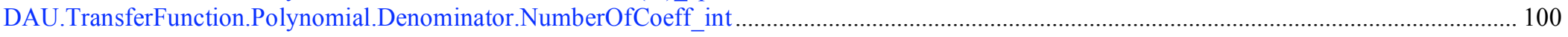

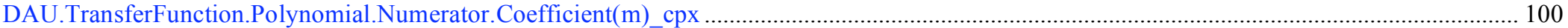

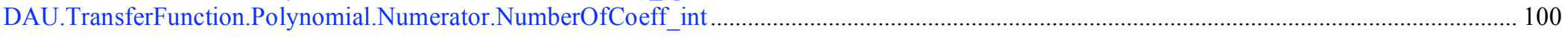

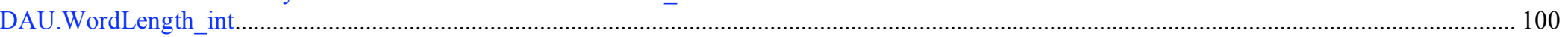

\section{Event}

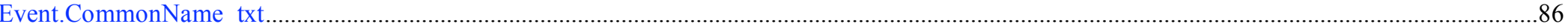

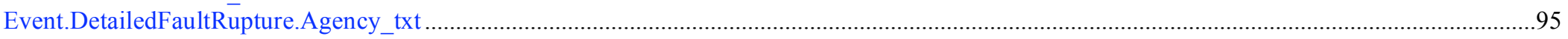

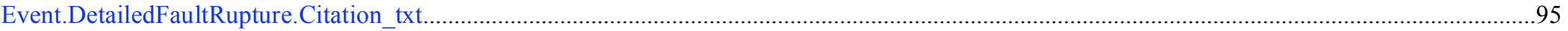

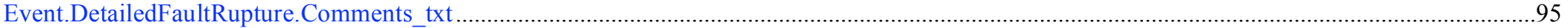

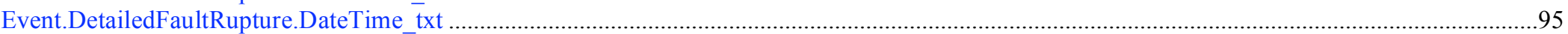

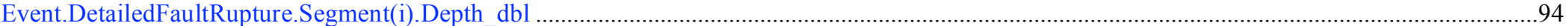

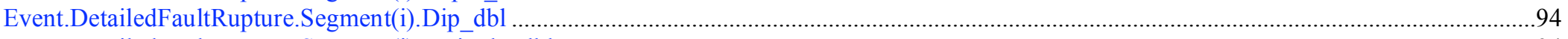

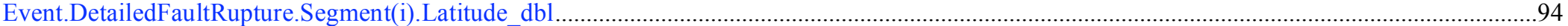

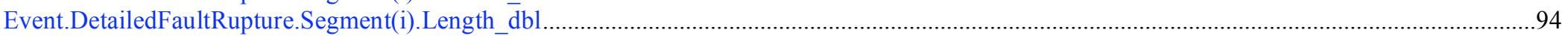

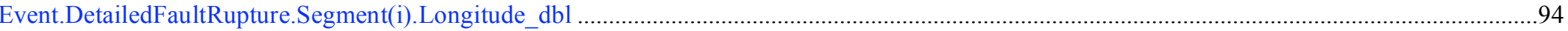

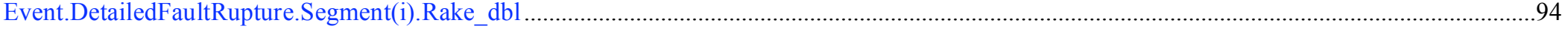

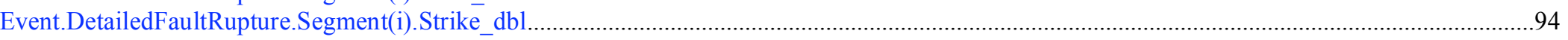

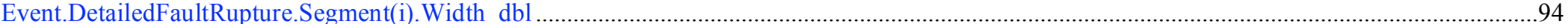

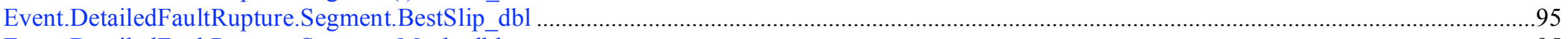

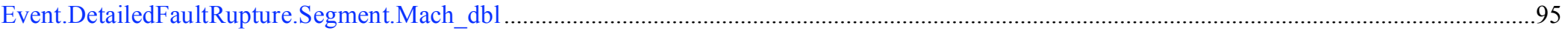

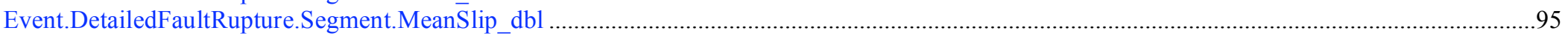

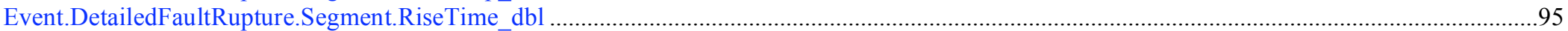




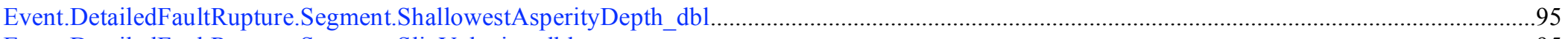

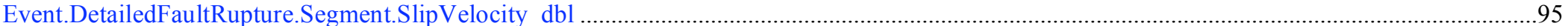

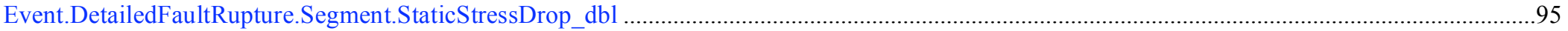

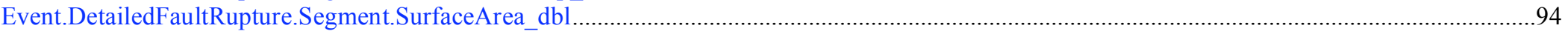

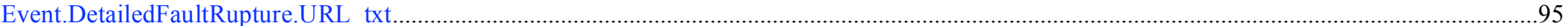

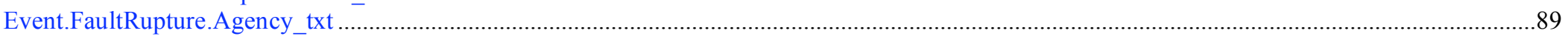

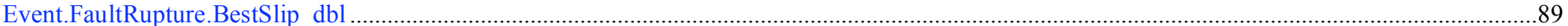

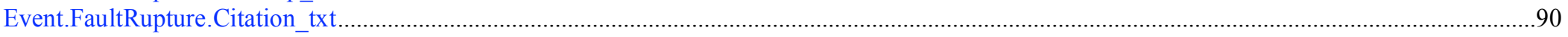

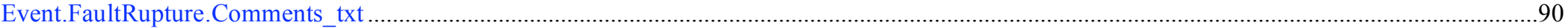

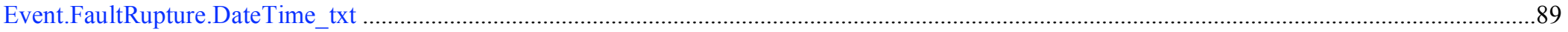

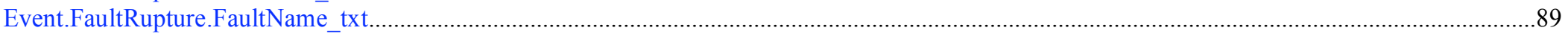

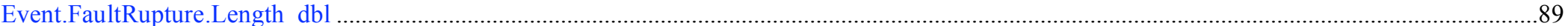

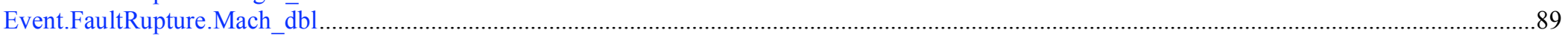

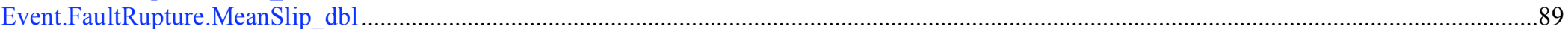

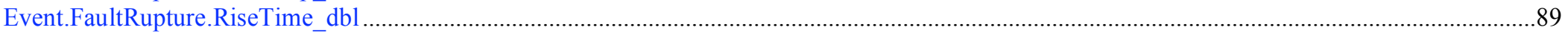

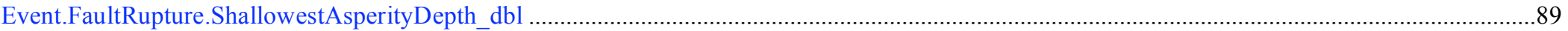

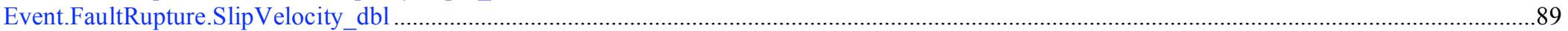

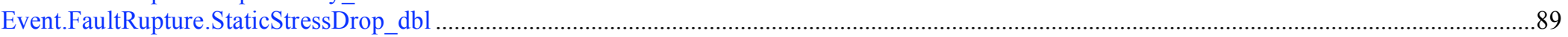

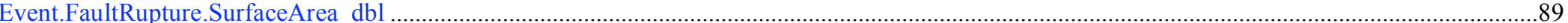

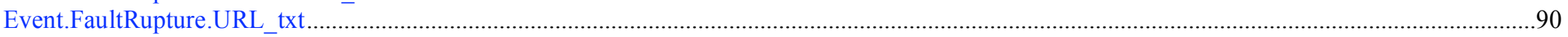

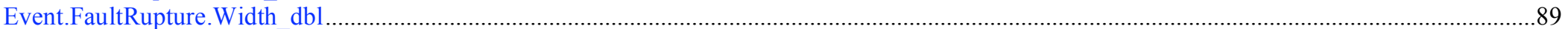

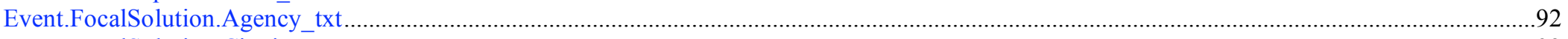

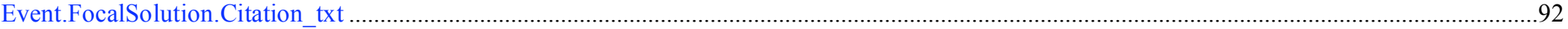

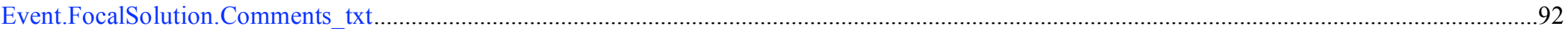

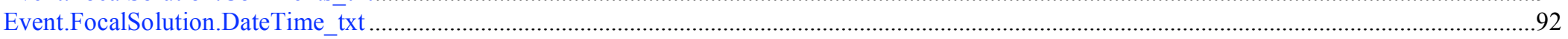

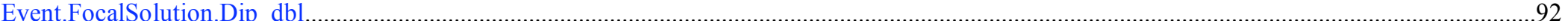

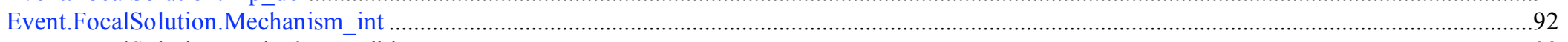

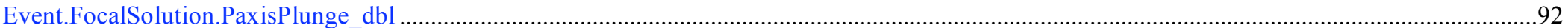

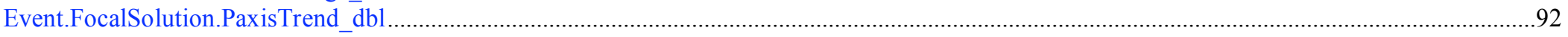

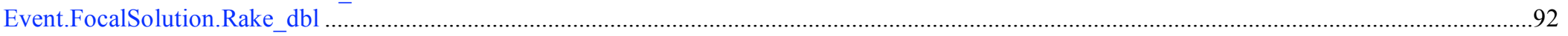

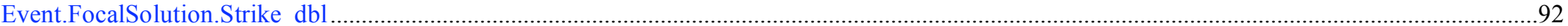

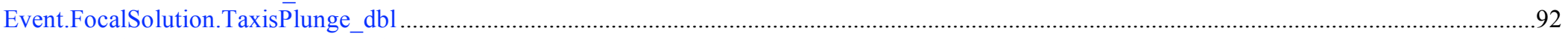

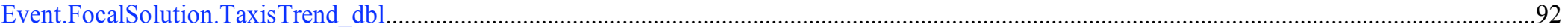

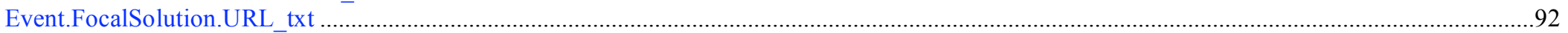

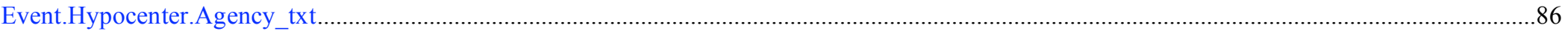

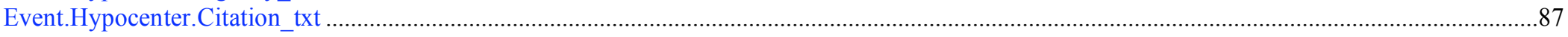

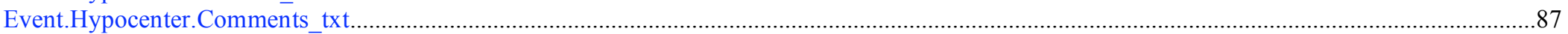




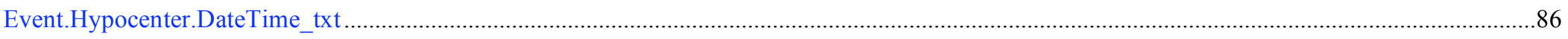

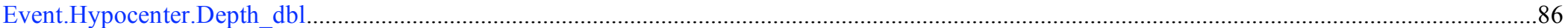

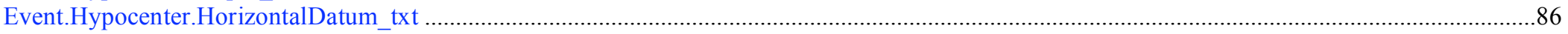

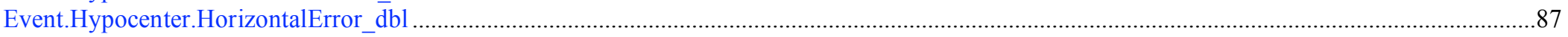

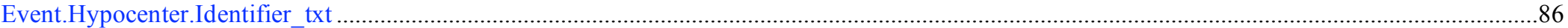

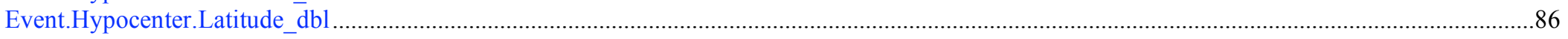

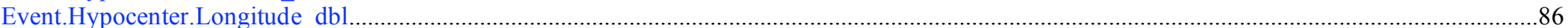

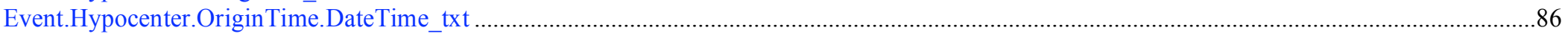

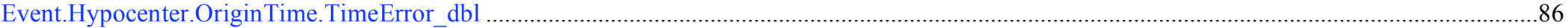

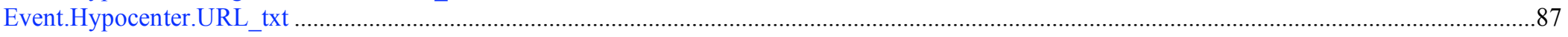

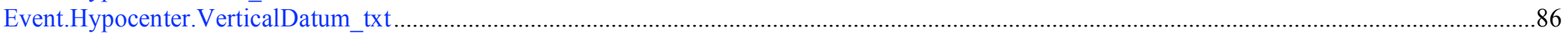

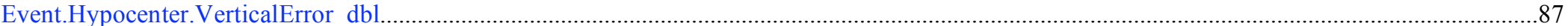

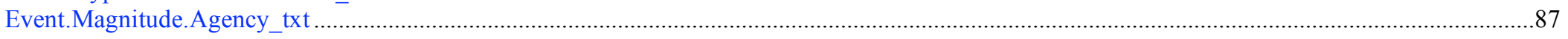

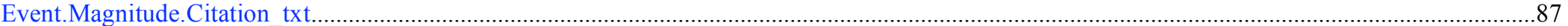

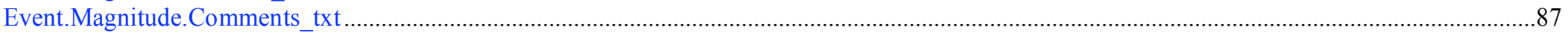

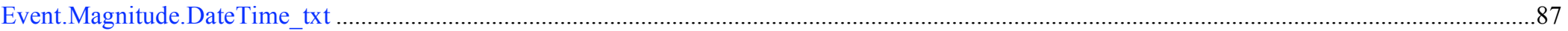

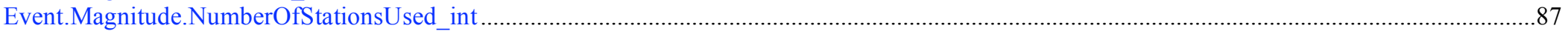

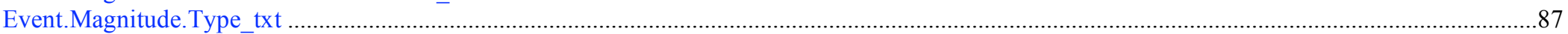

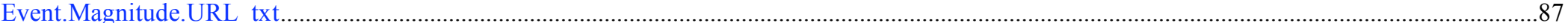

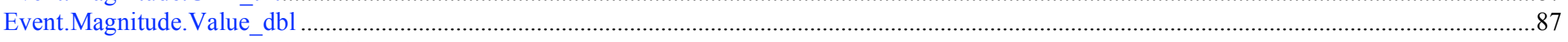

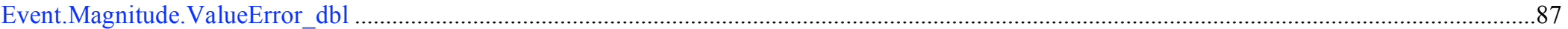

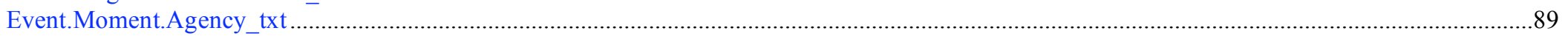

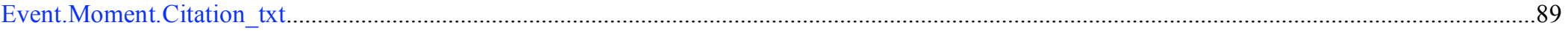

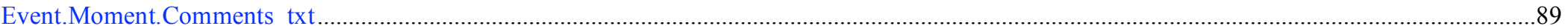

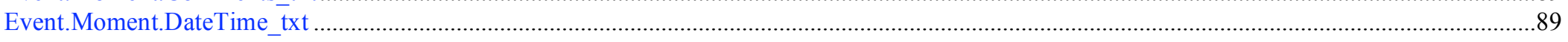

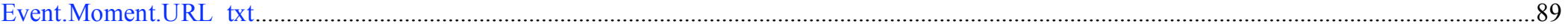

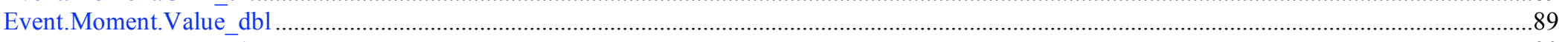

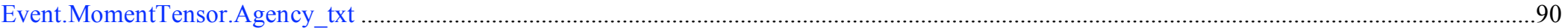

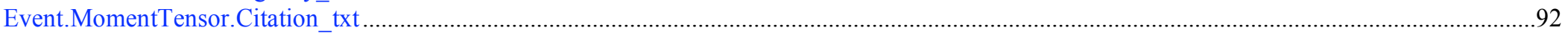

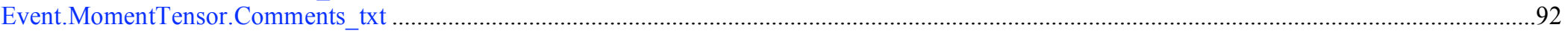

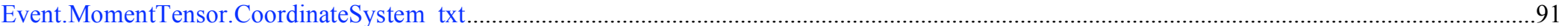

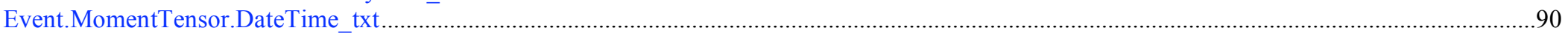

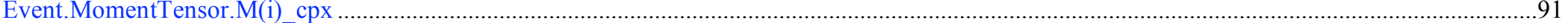

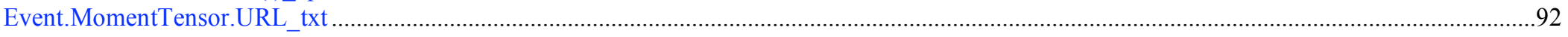

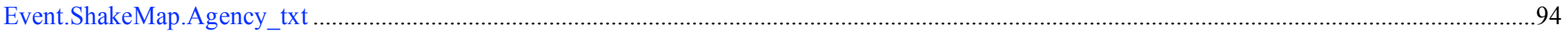

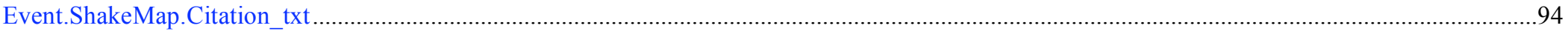

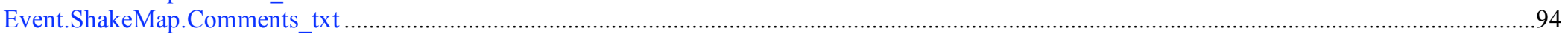




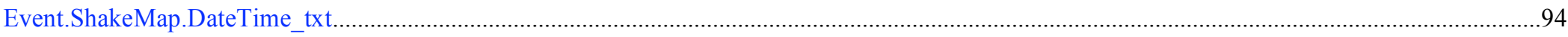

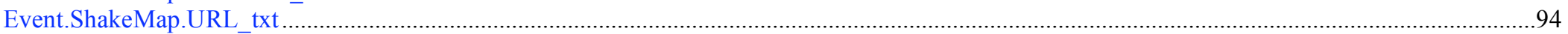

\section{EventGeoLocation}

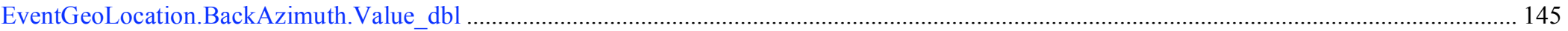

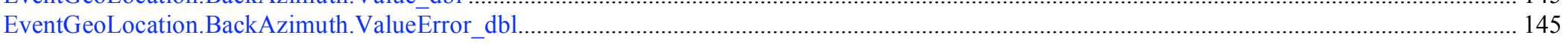

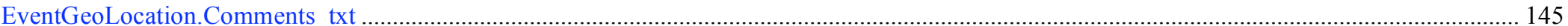

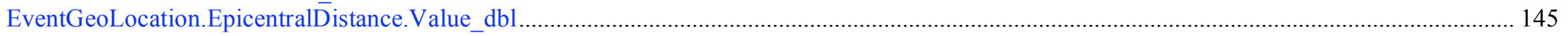

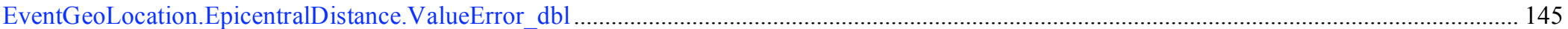

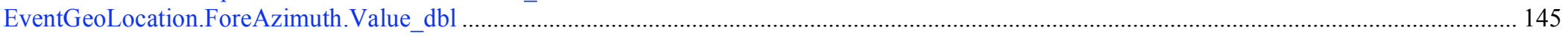

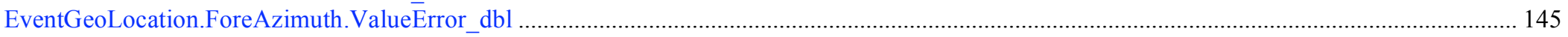

\section{GeoLocation}

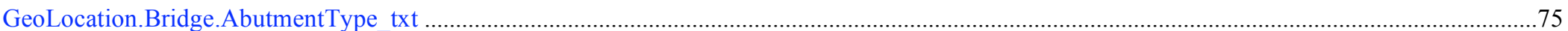

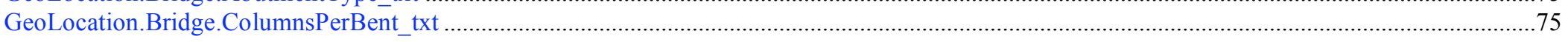

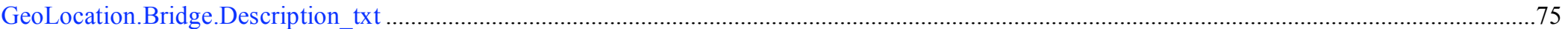

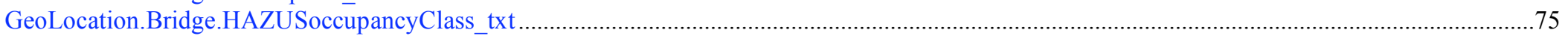

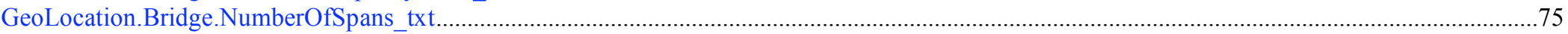

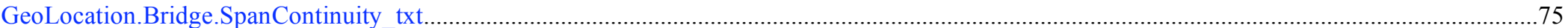

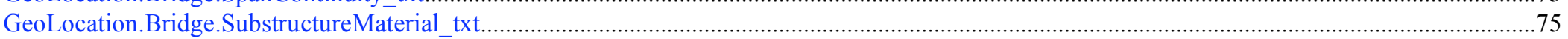

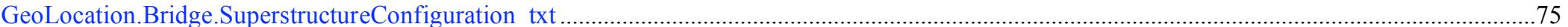

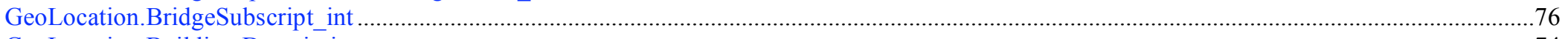

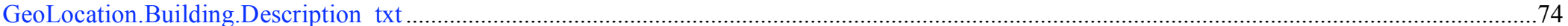

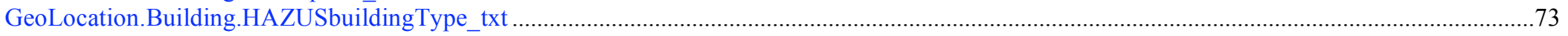

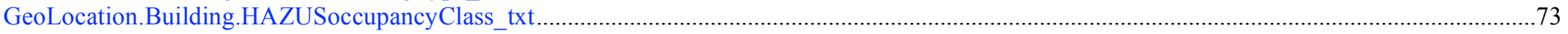

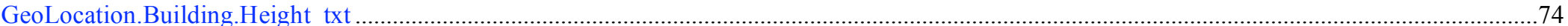

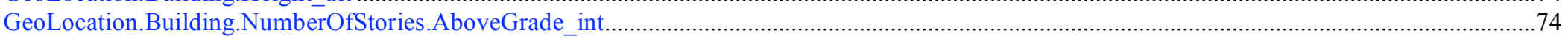

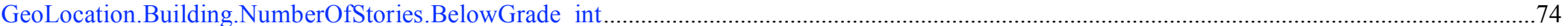

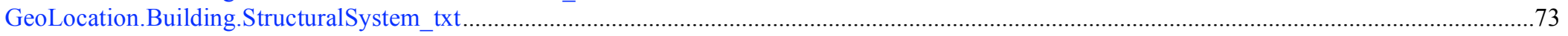

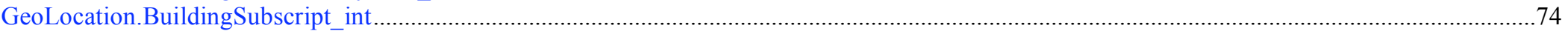

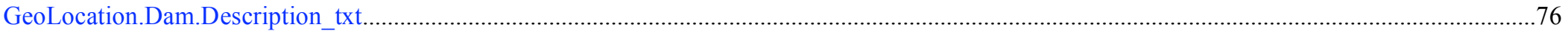

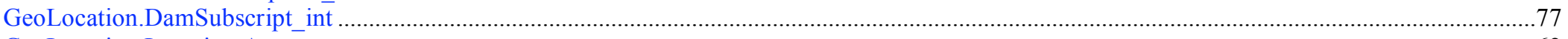

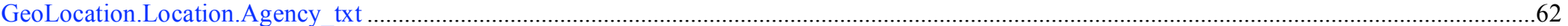

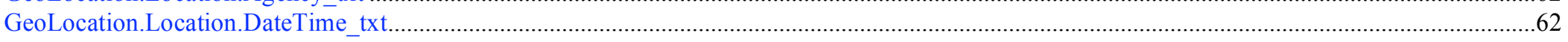

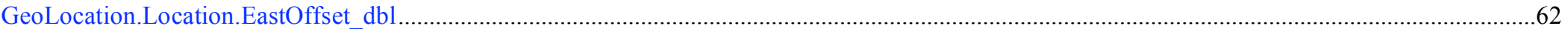

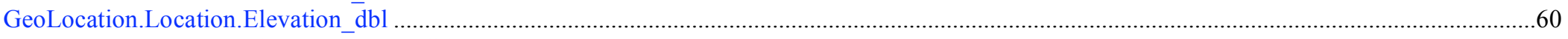

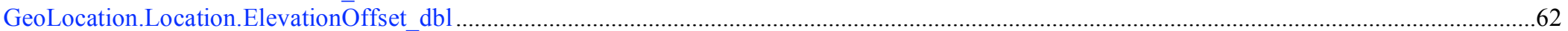




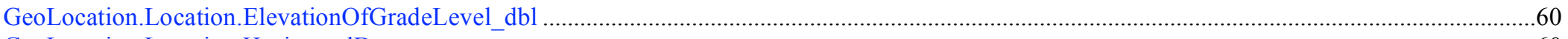

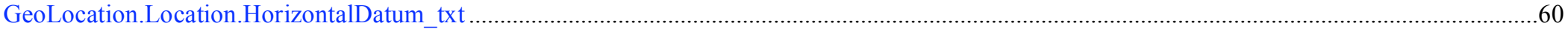

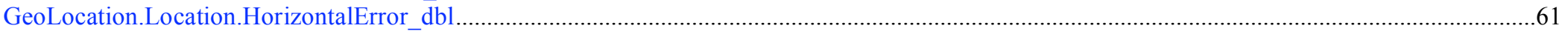

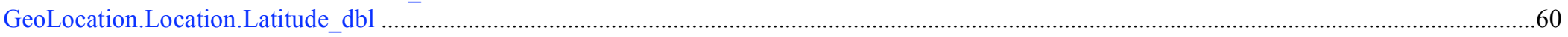

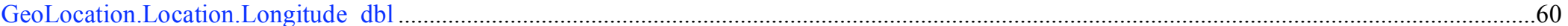

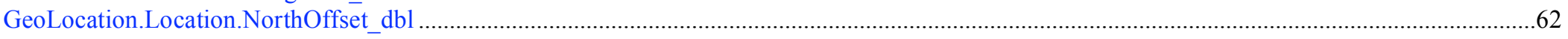

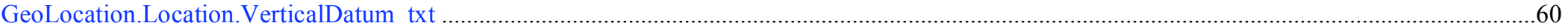

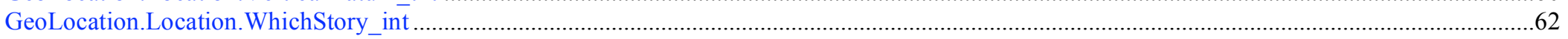

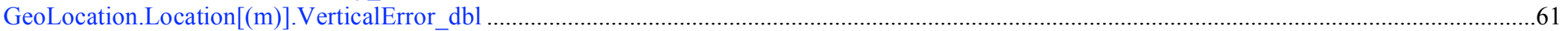

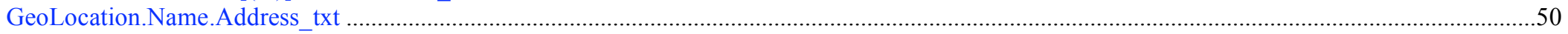

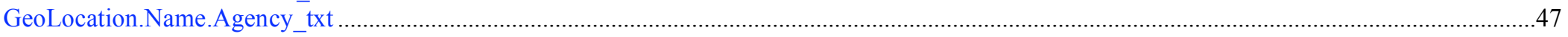

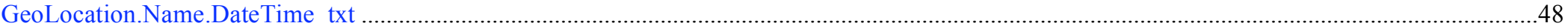

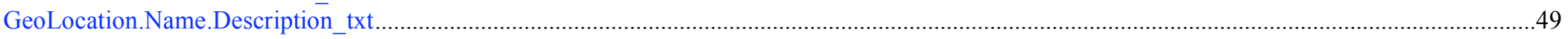

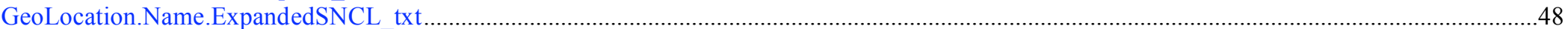

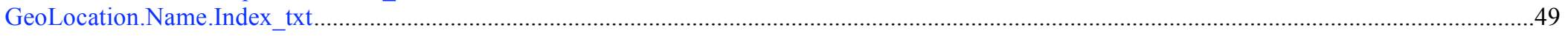

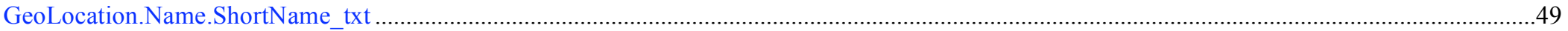

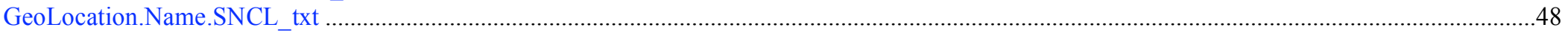

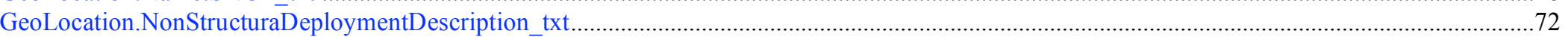

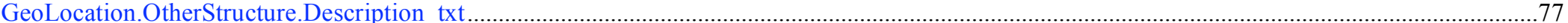

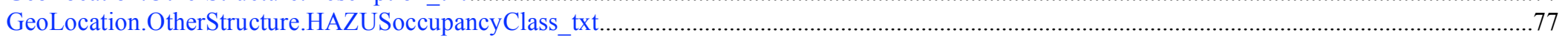

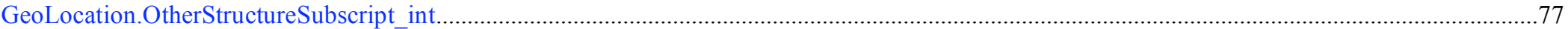

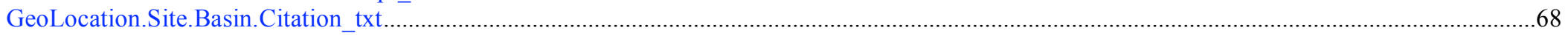

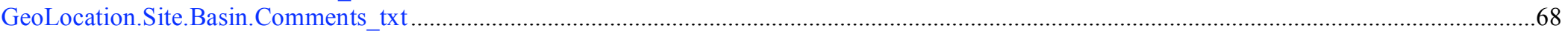

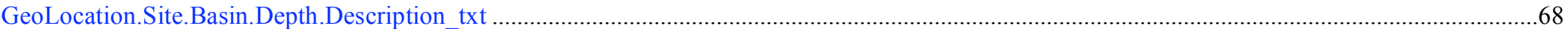

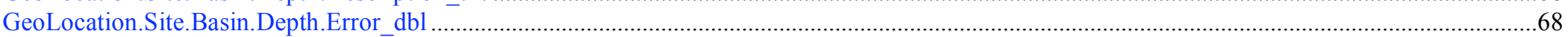

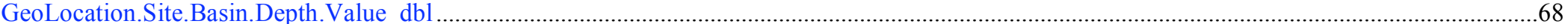

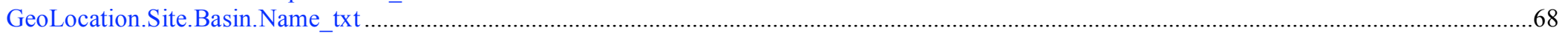

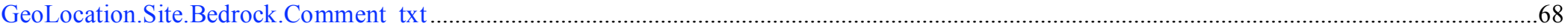

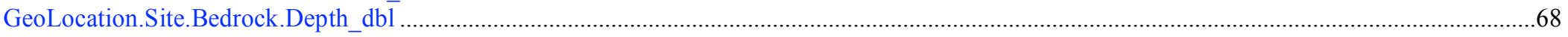

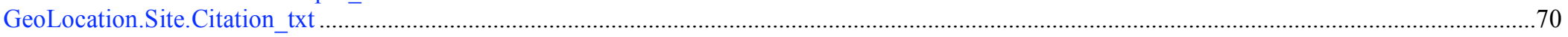

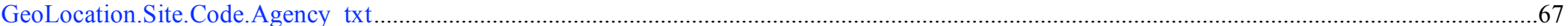

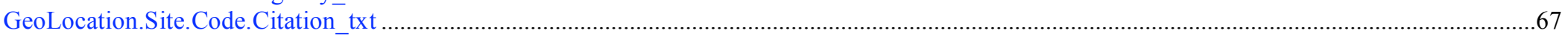

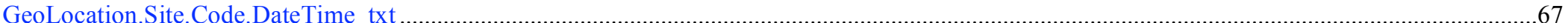

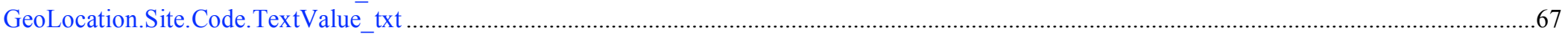

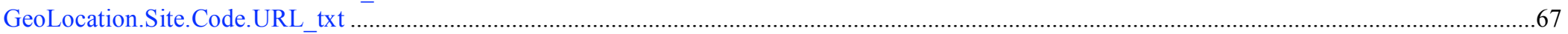

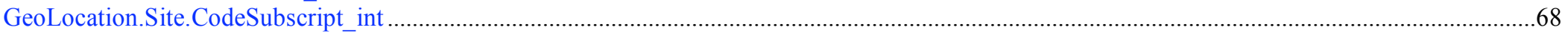

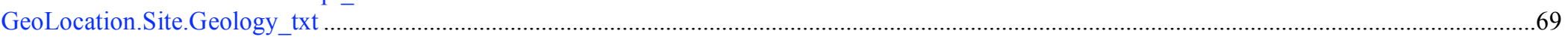




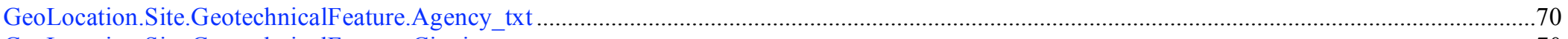

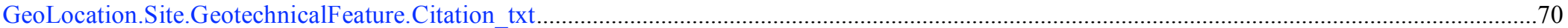

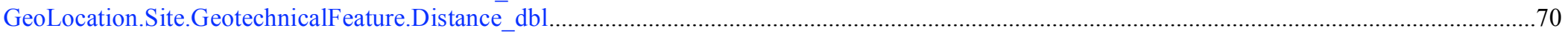

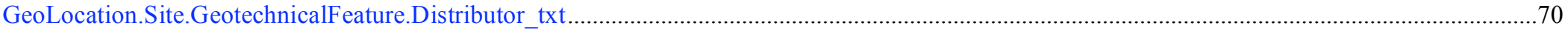

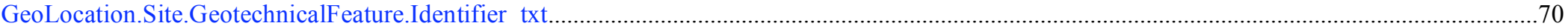

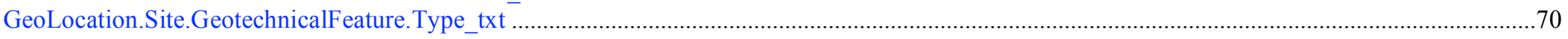

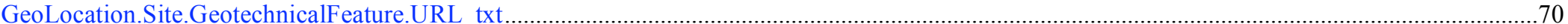

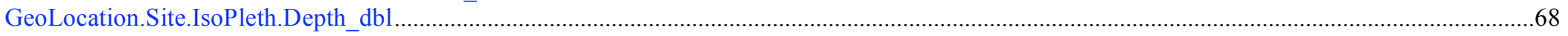

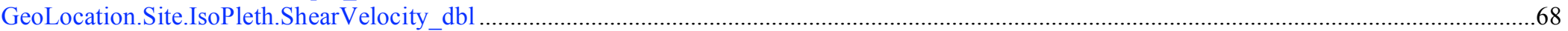

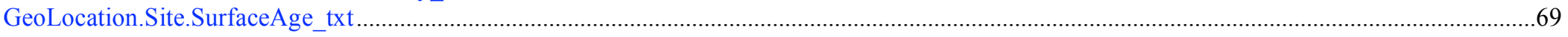

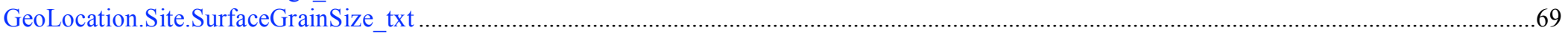

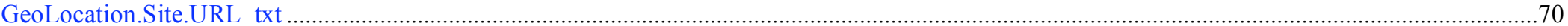

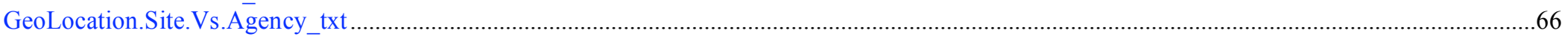

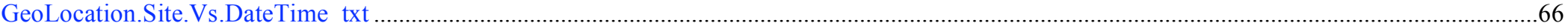

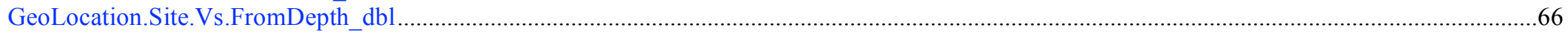

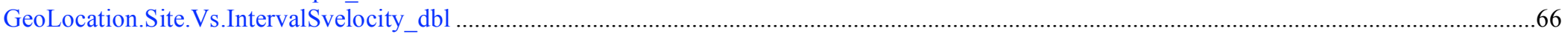

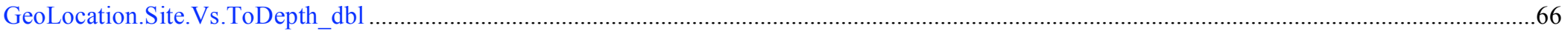

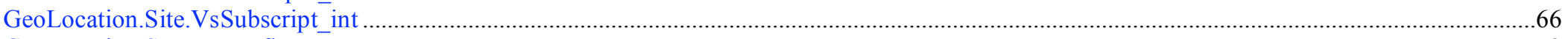

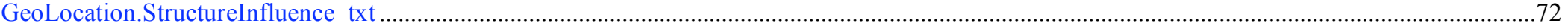

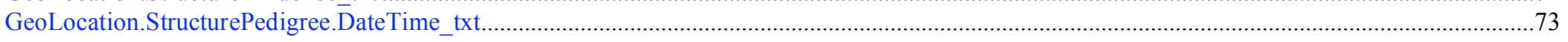

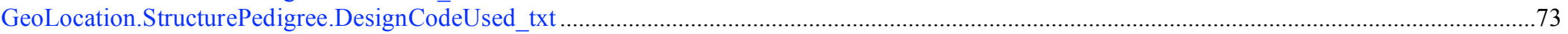

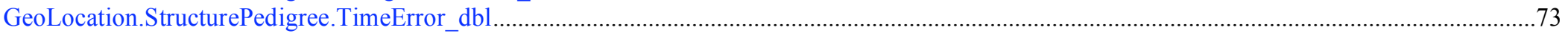

\section{GeoLocation Rupture}

GeoLocationRupture.ClosestRuptureDistance.Value_dbl

GeoLocationRupture.ClosestRuptureDistance.ValueError dbl $\ldots$

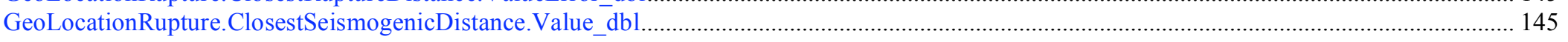

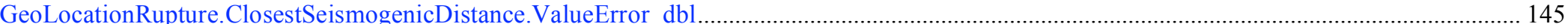

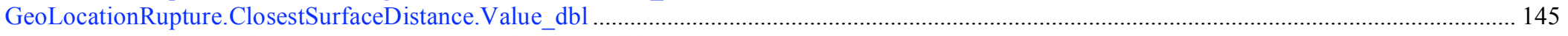

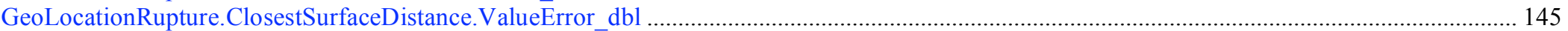

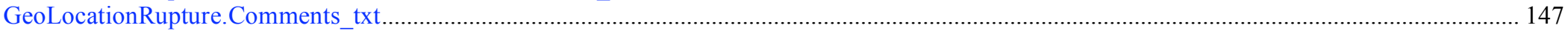

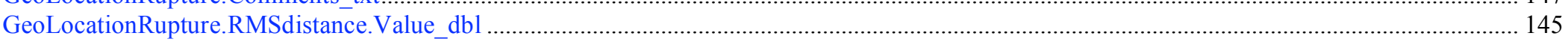

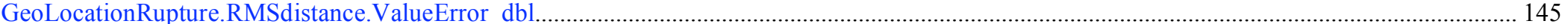

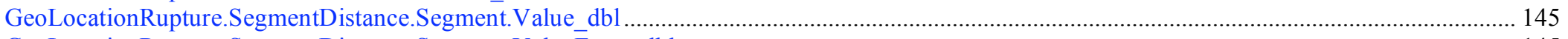

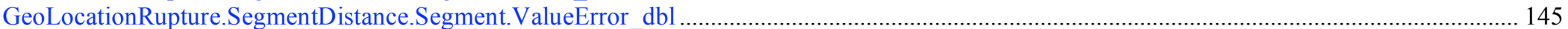

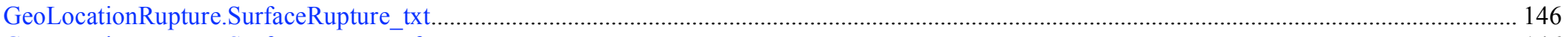

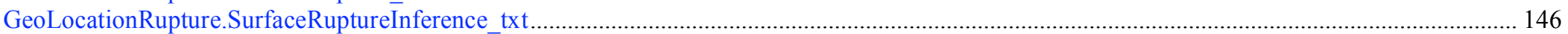




\section{Private}

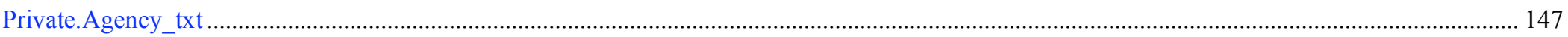

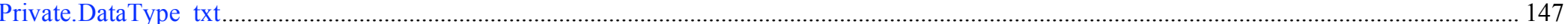

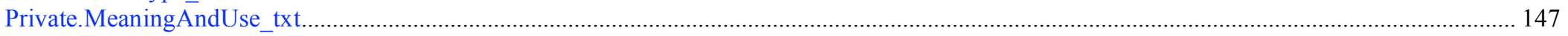

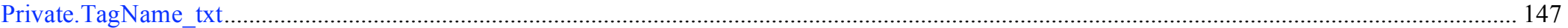

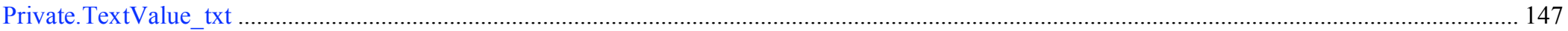

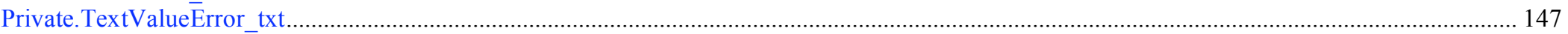

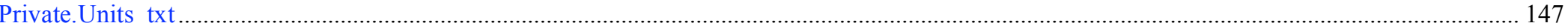

\section{Processing}

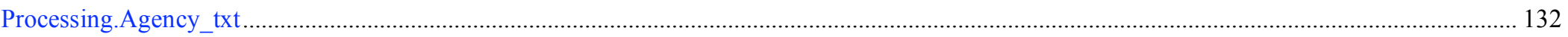

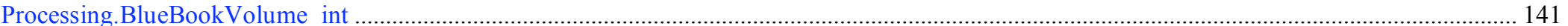

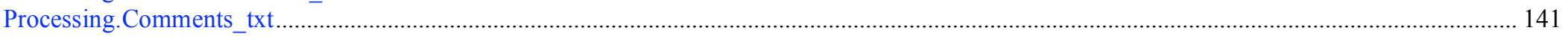

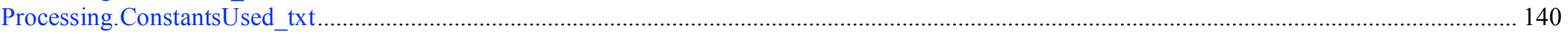

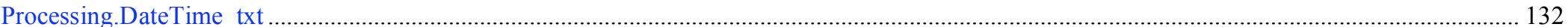

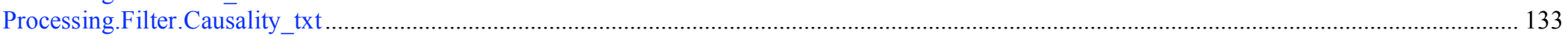

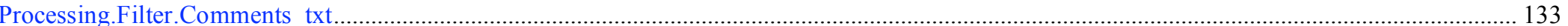

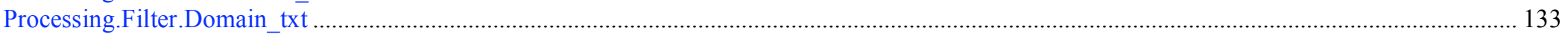

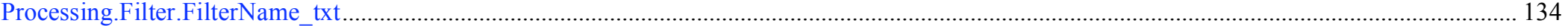

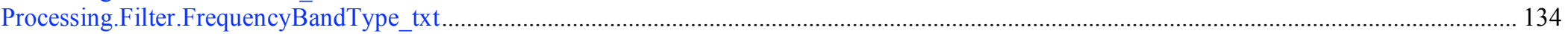

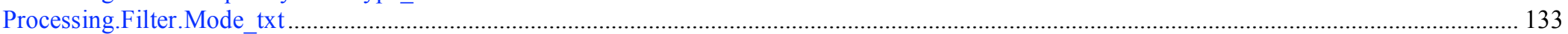

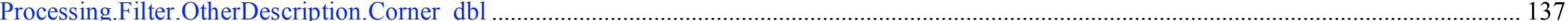

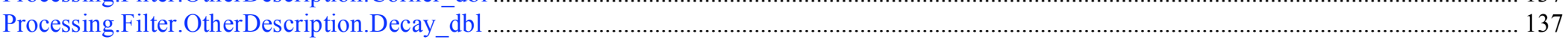

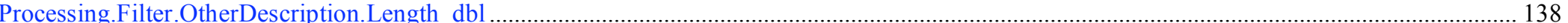

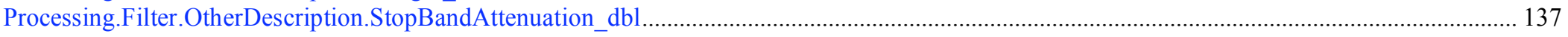

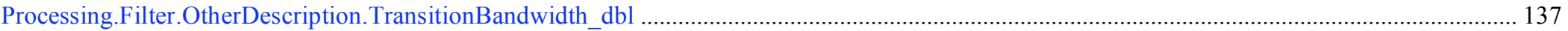

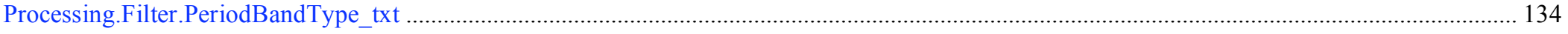

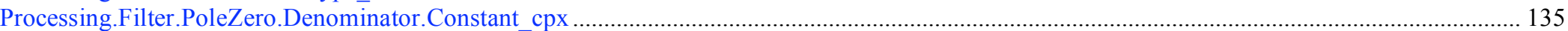

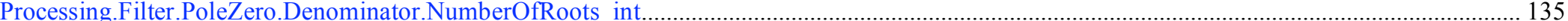

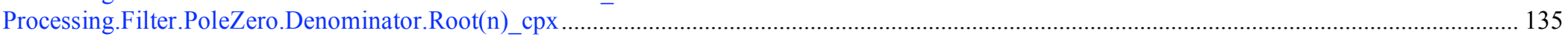

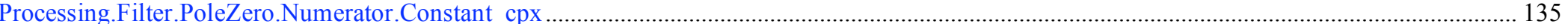

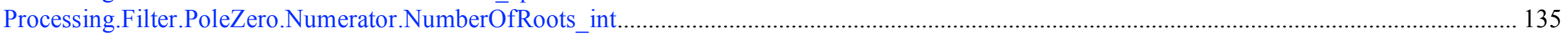

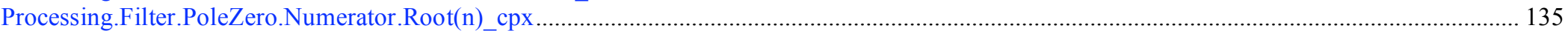

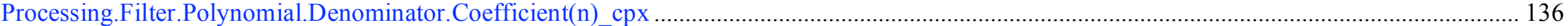

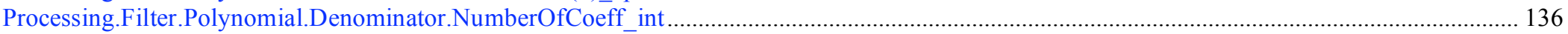

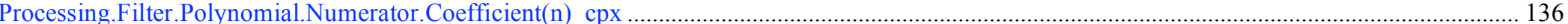

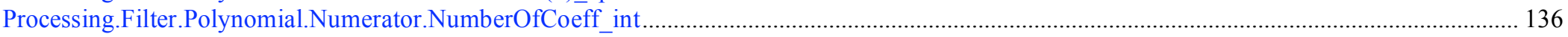




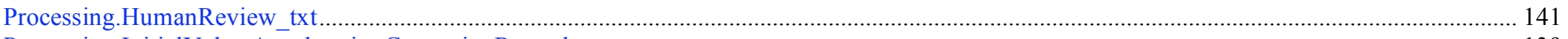

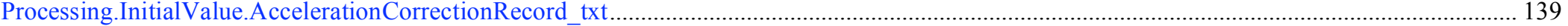

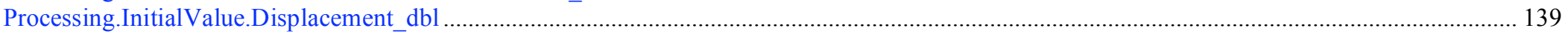

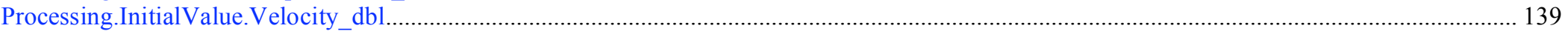

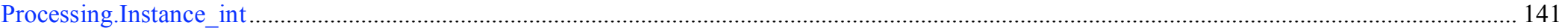

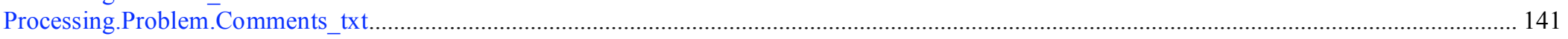

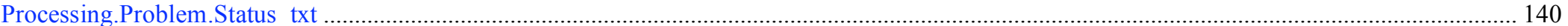

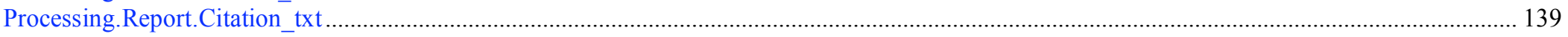

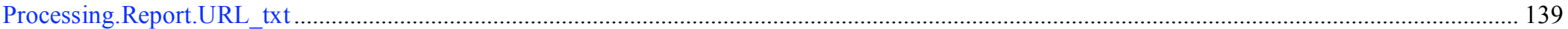

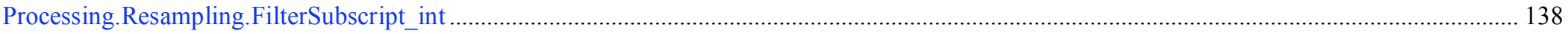

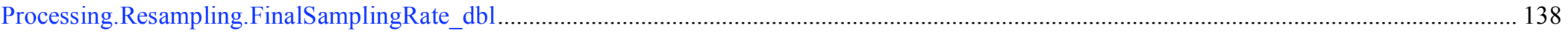

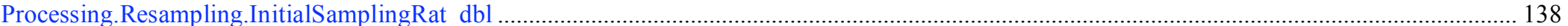

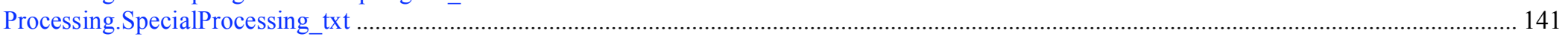

Processing.Stage txt............................ 141

\section{RawSeries}

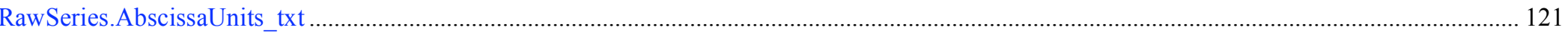

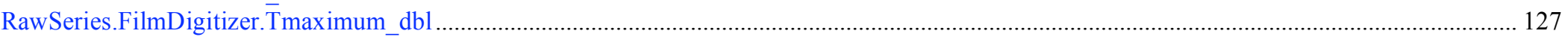

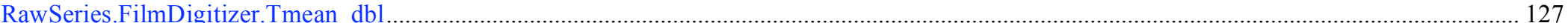

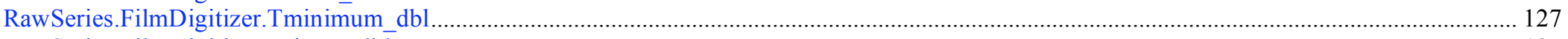

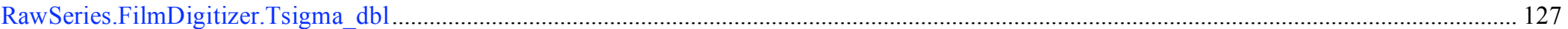

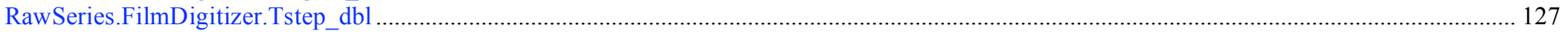

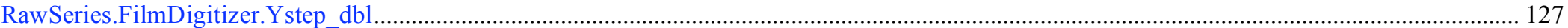

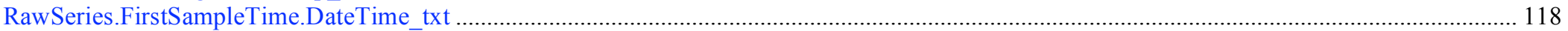

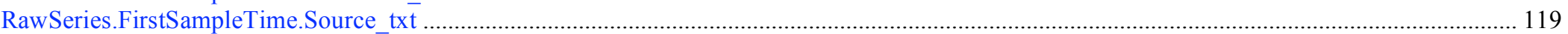

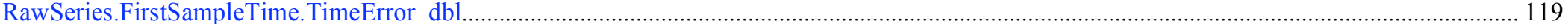

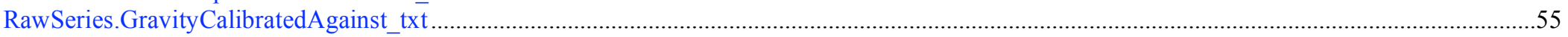

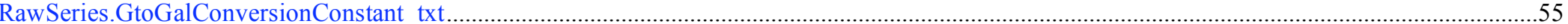

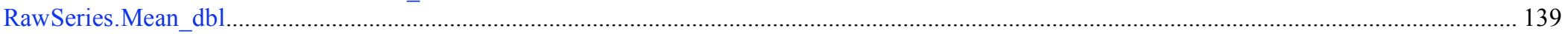

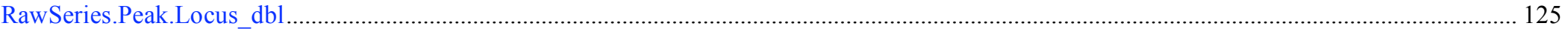

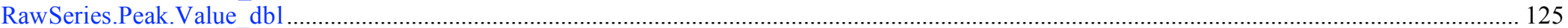

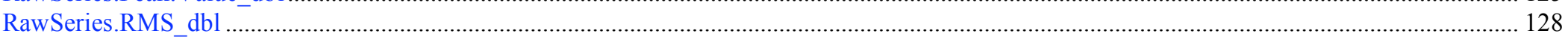

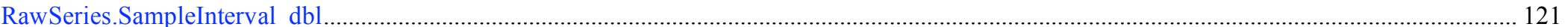

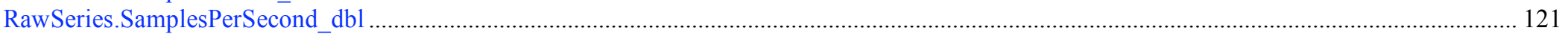

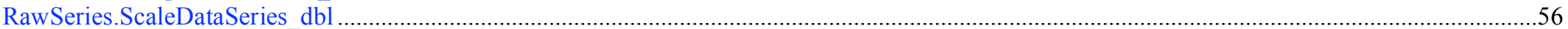

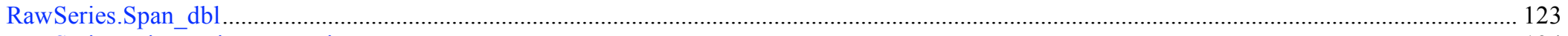

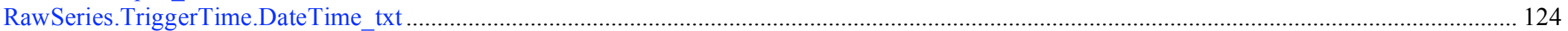


RawSeries.TriggerTime.TimeError_dbl

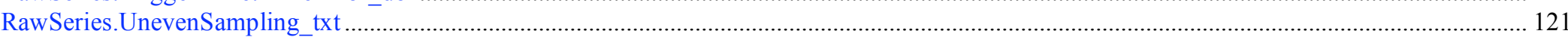

\section{Sensor}

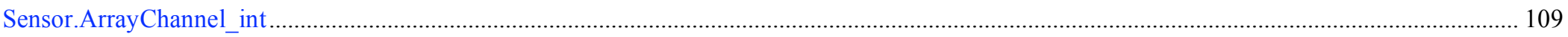

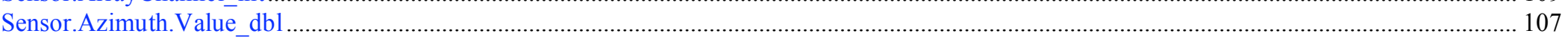

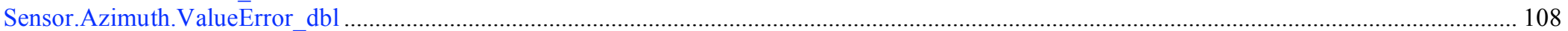

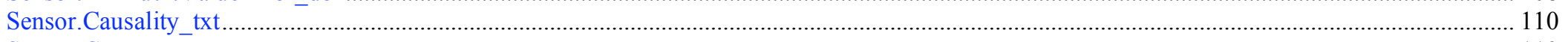

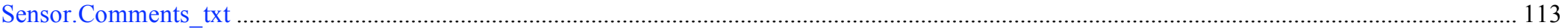

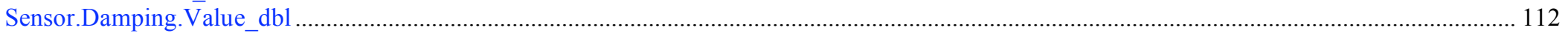

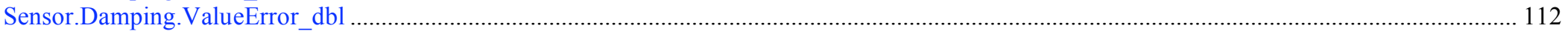

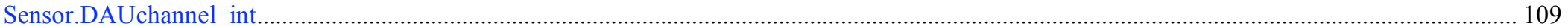

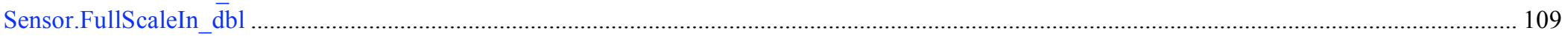

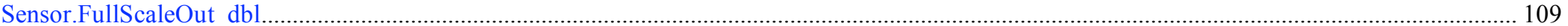

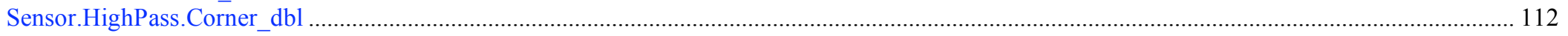

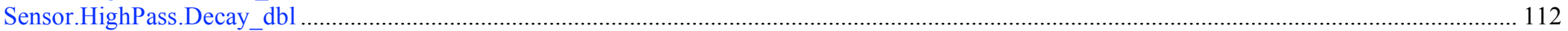

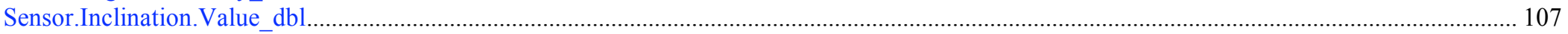

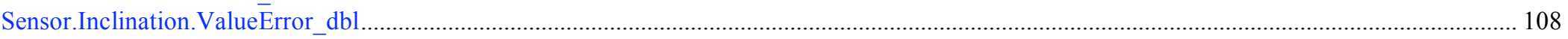

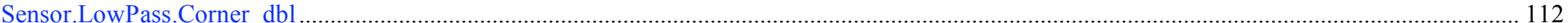

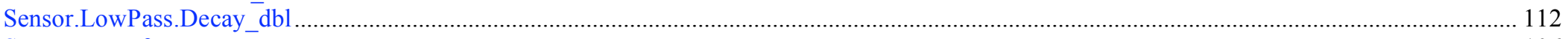

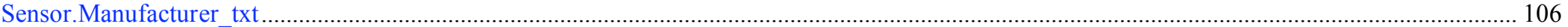

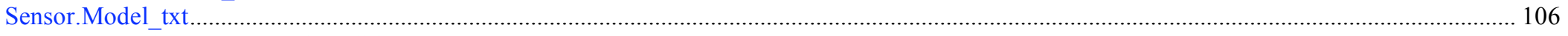

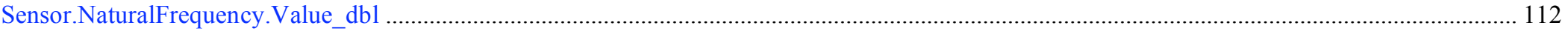

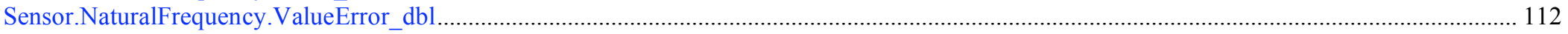

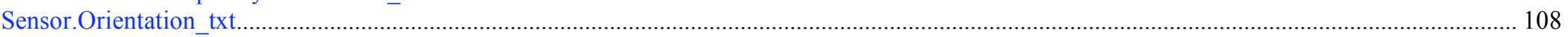

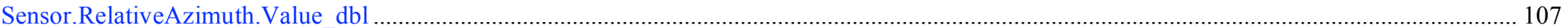

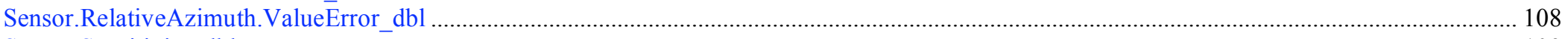

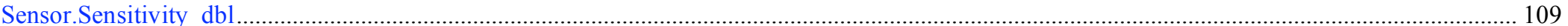

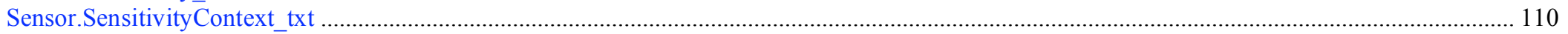

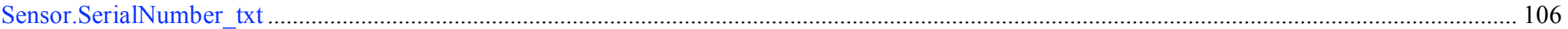

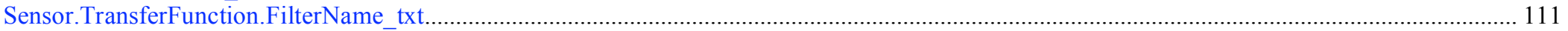

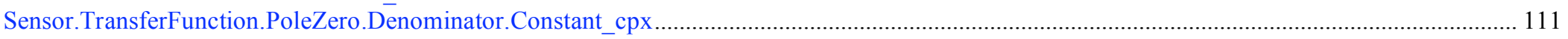

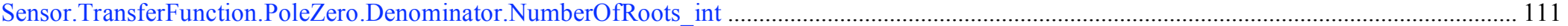

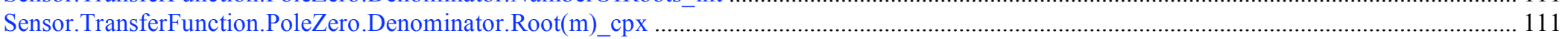

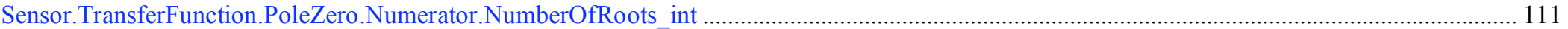

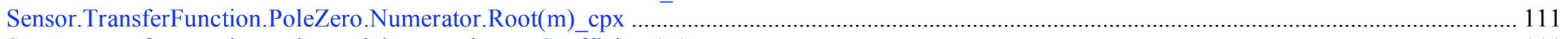

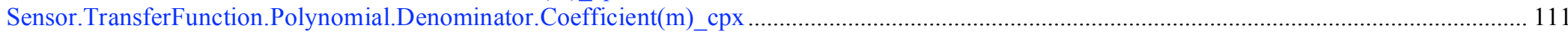


Sensor.TransferFunction.Polynomial.Denominator.NumberOfCoeff_int..................................................................................................... 111

Sensor.TransferFunction.Polynomial_Numerator_Coefficient $(\mathrm{m})$ cpx

Sensor.TransferFunction.Polynomial.Numerator.NumberOfCoeff_int................................................................................................................ 111

$S$ (cont.)

SubClassName .....

SubSubClassName

$T$

TagName

ThisFile

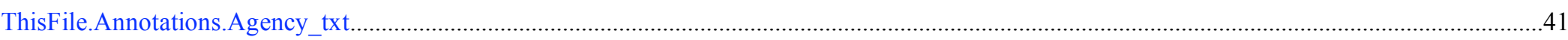

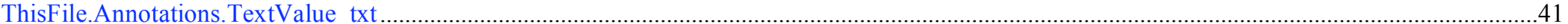

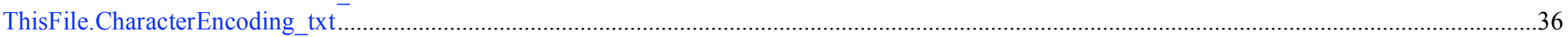

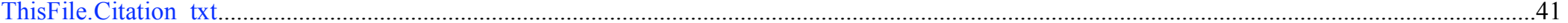

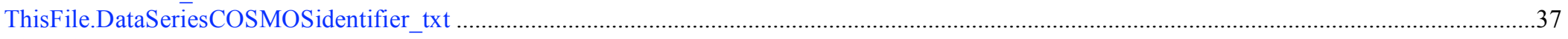

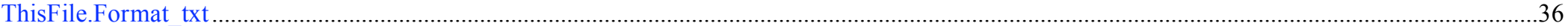

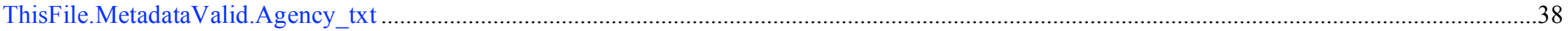

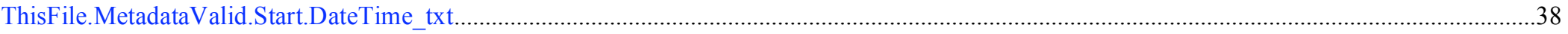

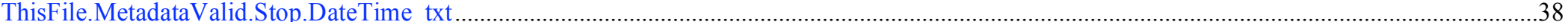

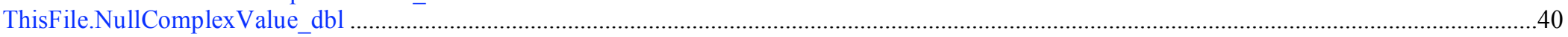

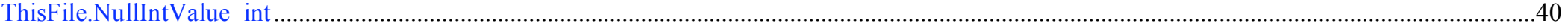

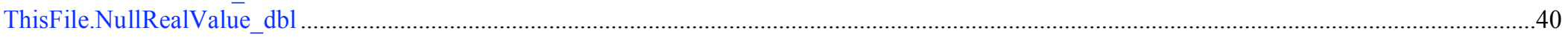

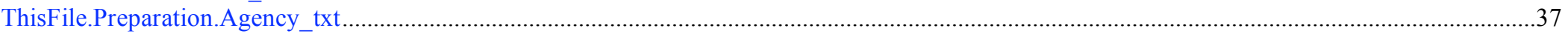

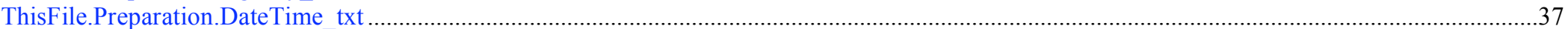

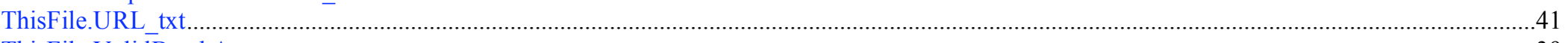

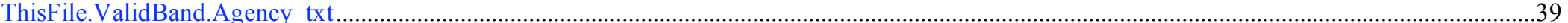

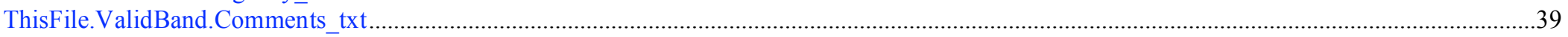

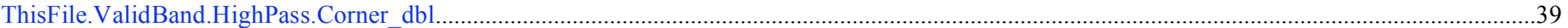

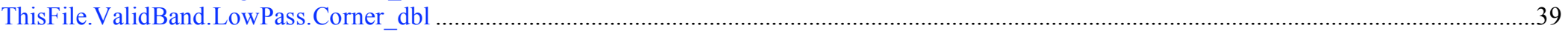

$V$

VariableType...... 